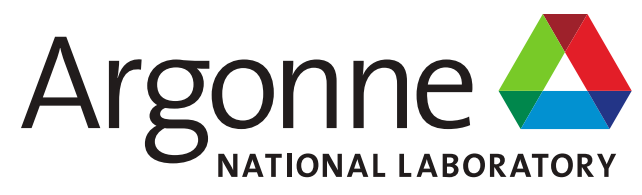

\title{
Transient Testing of Nuclear Fuels Performed in the Original Operation of TREAT
}

Second Edition

Chemical and Fuel Cycle Technologies Division Nuclear Science and Engineering Division 


\section{About Argonne National Laboratory}

Argonne is a U.S. Department of Energy laboratory managed by UChicago Argonne, LLC under contract DE-AC02-06CH11357. The Laboratory's main facility is outside Chicago, at 9700 South Cass Avenue, Argonne, Illinois 60439. For information about Argonne and its pioneering science and technology programs, see www.anl.gov.

\section{DOCUMENT AVAILABILITY}

Online Access: U.S. Department of Energy (DOE) reports produced after 1991 and a growing number of pre-1991 documents are available free at OSTI.GOV (http://www.osti.gov/), a service of the US Dept. of Energy's Office of Scientific and Technical Information.

Reports not in digital format may be purchased by the public from the National Technical Information Service (NTIS):

U.S. Department of Commerce

National Technical Information Service

5301 Shawnee Rd

Alexandria, VA 22312

www.ntis.gov

Phone: (800) 553-NTIS (6847) or (703) 605-6000

Fax: (703) 605-6900

Email: orders@ntis.gov

Reports not in digital format are available to DOE and DOE contractors from the Office of Scientific and Technical Information (OSTI):

U.S. Department of Energy

Office of Scientific and Technical Information

P.O. Box 62

Oak Ridge, TN 37831-0062

www.osti.gov

Phone: (865) 576-8401

Fax: (865) 576-5728

Email: reports@osti.gov

\section{Disclaimer}

This report was prepared as an account of work sponsored by an agency of the United States Government. Neither the United States Government nor any agency thereof, nor UChicago Argonne, LLC, nor any of their employees or officers, makes any warranty, express or implied, or assumes any legal liability or responsibility for the accuracy, completeness, or usefulness of any information, apparatus, product, or process disclosed, or represents that its use would not infringe privately owned rights. Reference herein to any specific commercial product, process, or service by trade name, trademark, manufacturer, or otherwise, does not necessarily constitute or imply its endorsement, recommendation, or favoring by the United States Government or any agency thereof. The views and opinions of document authors expressed herein do not necessarily state or reflect those of the United States Government or any agency thereof, Argonne National Laboratory, or UChicago Argonne, LLC. 
ANL-ART-186 Rev. 2

\section{Transient Testing of Nuclear Fuels Performed in the Original Operation of TREAT}

Second Edition

prepared by

A. E. Wright ${ }^{1}$ and C. A. Tomchik ${ }^{2}$

${ }^{1}$ Chemical and Fuel Cycle Technologies Division, Argonne National Laboratory

${ }^{2}$ Nuclear Science \& Engineering Division, Argonne National Laboratory

August 2020 
(This page left intentionally blank) 


\section{Executive Summary}

This report provides summary information on experiments performed in the Transient Reactor Test Facility (TREAT) during its original operation from 1959-1994, and more specifically, before the TREAT core was upgraded at the end of the 1980s. The tests performed since about 1970 are summarized in considerable detail. Earlier tests are noted by their designated series and by some parameters that help to identify and distinguish each series from the others. The detailed summary descriptions are intended to help inform future researchers regarding the large, historical, empirical data base generated from that 35-year span during which hundreds of TREAT experiments were performed to investigate and demonstrate the transient behavior of a wide variety of nuclear fuels during severe off-normal and accident conditions -- using direct nuclear heating of the test samples. That empirical basis is foundational and valuable for guiding future qualification and safety evaluation of improved fuel designs and for future transient test planning and performance in TREAT. Since Edition 1 of this report, descriptions of the following test series have been added: D, E, EOS, F, $\mathrm{H}, \mathrm{J}, \mathrm{R}, \mathrm{RX}$, and $\mathrm{S}$. 


\section{Table of Contents}

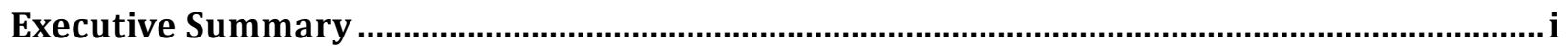

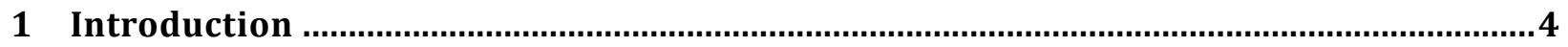

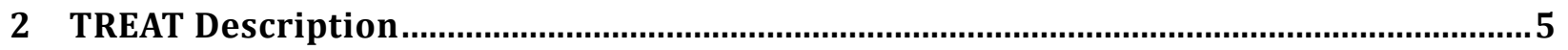

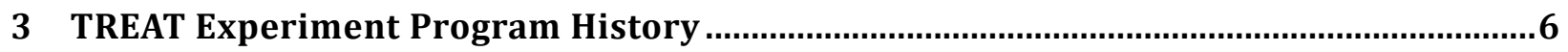

4 Historical Experiment Series Included in This Report ........................................................

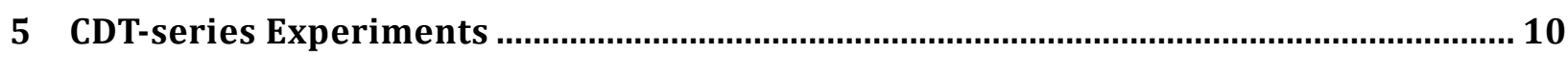

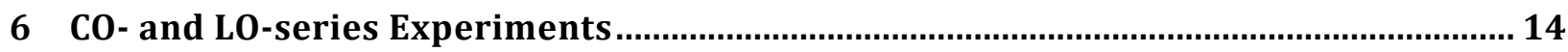

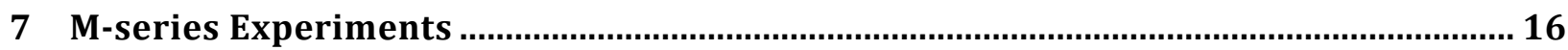

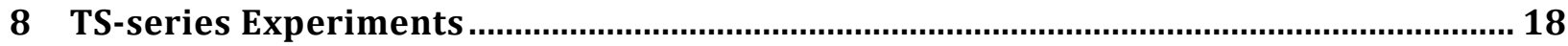

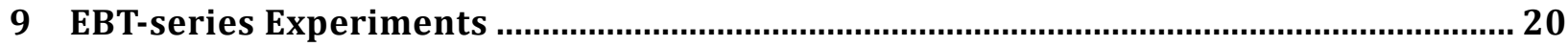

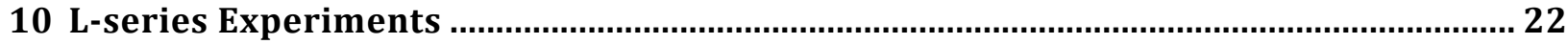

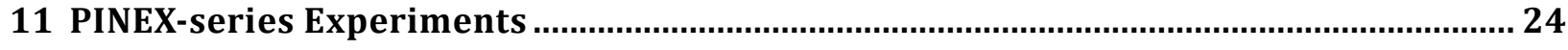

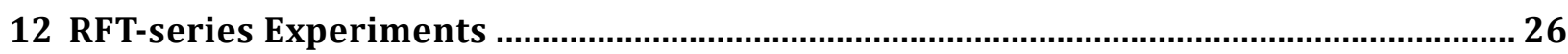

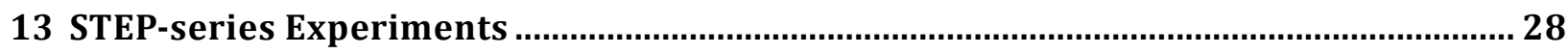

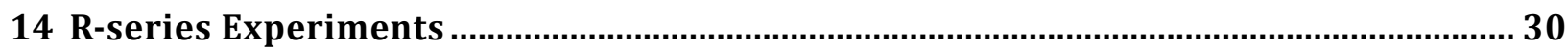

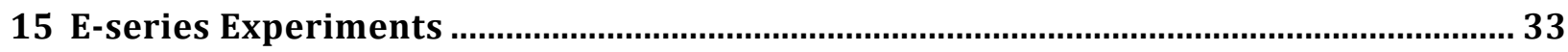

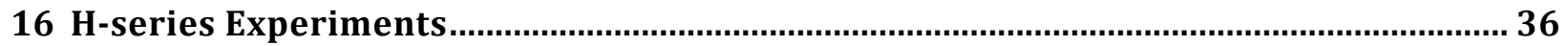

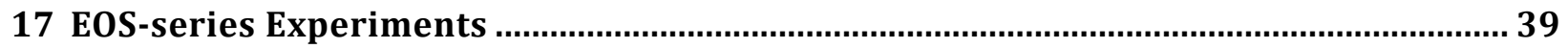

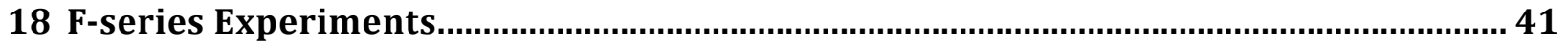

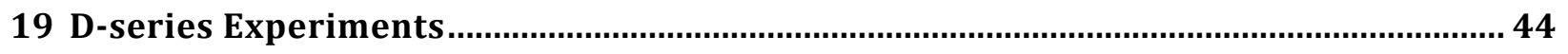

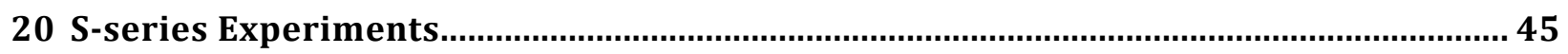

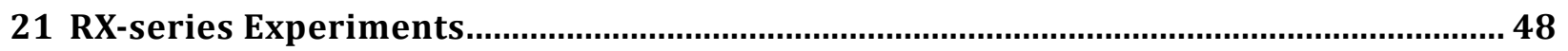

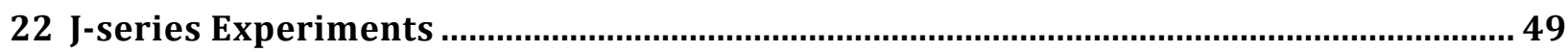

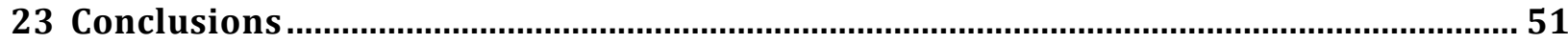

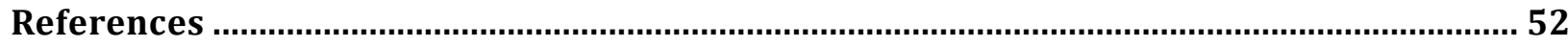

Appendix A: List of All Experiment Series ........................................................................... 59

Appendix B: One-page Summaries of CDT-series Tests...................................................... 63

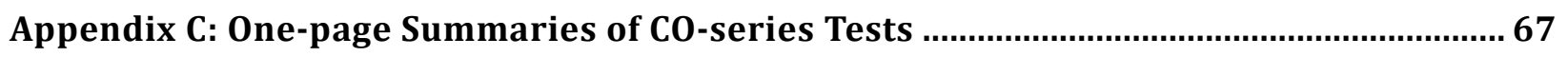

Appendix D: One-page Summaries of LO-series Tests ........................................................... 74

Appendix E: One-page Summaries of M-series Tests ........................................................ 82

Appendix F: One-page Summaries of TS-series Tests ............................................................... 90

Appendix G: One-page Summaries of EBT-series Tests .......................................................... 93

Appendix H: One-page Summaries of L-series Tests.............................................................. 98

Appendix I: One-page Summaries of PINEX-series Tests .............................................107 
Appendix J: One-page Summaries of RFT-series Tests …...........................................110

Appendix K: One-page Summaries of STEP-series Tests.....................................................115

Appendix L: One-page Summaries of R-series Tests .......................................................120

Appendix M: One-page Summaries of E-series Tests ........................................................129

Appendix N: One-page Summaries of $\mathrm{H}$-series Tests ...........................................................137

Appendix 0: One-page Summaries of EOS-series Tests ...................................................144

Appendix P: One-page Summaries of F-series Tests ..........................................................148

Appendix Q: One-page Summaries of D-series Tests .......................................................154

Appendix R: One-page Summaries of S-series Tests ...........................................................157

Appendix S: One-page Summaries of RX-series Tests ..........................................................167

Appendix T: One-page Summaries of J-series Tests .........................................................170 


\section{Introduction}

The Transient Reactor Test Facility (TREAT) is an experimental reactor designed for the transient testing of nuclear reactor fuels and other materials [1]. TREAT was designed and built by Argonne National Laboratory (ANL) at a site that is now the Idaho National Laboratory (INL) [2]. ANL (as a collaboration between ANL-East and ANL-West) operated TREAT for use in hundreds of experiments from 1959 until 1994, when the reactor was placed in non-operational standby. In 2013, the United States Department of Energy (U.S. DOE) identified the need for a resumption of transient testing in the United States to support the development and qualification of new, advanced nuclear reactor fuels [3] and decided to restart TREAT for that purpose. TREAT was sucessfully restarted by INL in November 2017 [4]. Moving forward, TREAT will be operated by INL to support new transient experiment programs.

Future operation of TREAT will benefit from the information produced during the extensive prior utilization of the facility, which included a wide variety of experiments. Knowledge of those prior experiments, including how they helped to develop the ability to understand and predict the transient behaviors of novel, advanced fuels, provides a foundation for qualification of numerous fuel forms already tested, as well as planning and designing new future tests [5].

The TREAT test series for which detailed summaries for this report were prepared were selected from among the more than five dozen historical TREAT test series using the following criteria: (1) tests that are the most recent, and therefore pertain to the more modern fuel designs and experimental approaches and address the issues that were the most current near the end of that historical period, (2) tests that illustrate the range of interdependent capabilities of the TREAT reactor and of the inpile experiment systems designed to operate within the TREAT core, (3) tests that span the range of experiment outcomes ranging from mild fuel changes without cladding breach to gross fuel meltdown and dispersal of molten fuel and cladding materials, (4) tests of the main fuels (oxides and metallic) of interest during the historical period, and (5) tests comprising series that relate to one another in terms of their considerable transient impact on their test fuel samples.

It is envisioned that subsequent editions of this report could address the remaining approximately 40 test series in the following groupings: mild-transient tests performed on reference full-length FFTF oxide fuel, tests performed on carbide and nitride fuels during the 1970s and earlier, and the tests performed prior to the late 1960s. (To efficiently address the last group, which includes an extraordinarily large number of tests, many of which were relatively simple, summaries would not be prepared for each test individually but by sub-groups within their series.)

A brief summary of the entire set of historic TREAT experiments is provided in Appendix A. Further information on the broader set of experiments performed in TREAT can be found in the TREXR (TREAT Experimental Relational) Database, a compendium of reference documents and data which has been developed over the past several years at ANL [6].

In addition to the selected experiment set summaries, this report also provides brief background information on the TREAT reactor and the general nature of TREAT experimentation. 


\section{TREAT Description}

TREAT is a graphite-moderated, graphite-reflected, air-cooled reactor fueled with $93 \%$ enriched $\mathrm{UO}_{2}$ dispersed in graphite. The fuel is arranged in approximately four-inch-square fuel elements consisting of a $\sim$ four-foot-long zircaloy-clad central fuel region and $\sim$ two-foot-long aluminum-clad graphite reflector regions above and below the fuel region. The reactor core can accommodate a maximum of 361 TREAT fuel elements in a 19x19 array. For irradiation experiments, a small number (typically two, occasionally a few more) of central TREAT fuel elements are replaced with an experiment vehicle containing the test sample(s). Frequently, an additional row (or half row) of fuel assemblies is also removed to provide an unimpeded path for fast neutron travel between the test sample and an ex-core system of collimator and detectors, collectively called the TREAT Fast Neutron Hodoscope. The hodoscope was used to provide real-time monitoring of fast neutrons emanating from test fuel samples located at the core center.

The core is controlled by $\mathrm{B}_{4} \mathrm{C}$-bearing control rods, arranged in three banks:

1. Control/shutdown rods, used to set the reactor to a critical state,

2. Computer-controlled transient rods, capable of high-speed travel, used to introduce reactivity changes which drive TREAT transients, and

3. Compensation/shutdown rods, used to compensate for the reactivity addition which occurs when a TREAT experiment vehicle is removed.

The core is cooled by forced air flow provided by two blowers. Because of the short duration of most power transients, the cooling effect is insignificant during the transients. Cooldown after transients typically takes several hours. The reactor core is enclosed in a radial graphite reflector surrounded by a concrete bioshield. Both the reflector and bioshield have holes to accommodate instrumentation.

TREAT transients are performed by introducing reactivity changes via movement of the transient rods from a critical pre-transient configuration (in which the transient and control/shutdown rod banks are both partially inserted within the core). There are three general categories of TREAT transients:

1. Temperature-limited ("burst") transients, in which a quick ("step") insertion of reactivity is caused by withdrawing the transient rods at their maximum speed, resulting in a bell-shaped burst in power which is constrained by the large, prompt negative temperature reactivity feedback provided by the heating of the TREAT fuel graphite;

2. Shaped transients, in which complex motions of the transient rods, under full computer control, are used to produce a specific core power-time history different from a simple power burst; and

3. Extended transients, lasting for several minutes at relatively low power, during which the reactor power is controlled by a combination of manual adjustment of control rod positions and computer control of transient rod positions. (Extended transients were historically used only in the STEP series of experiments which were performed in the mid-1980s.)

Major upgrading of the facility in the late 1980s included changing the locations of the control and transient rod banks in the core. The current, symmetrical arrangement, with 18 control rods and each of the three independent control rod sets operated simultaneously as a "bank," is referred to as the "upgraded core." The previous configuration, which was not symmetric, had fewer rods, and used two pairs of transient rods operated sequentially, is called the "pre-upgrade core." The changed configuration resulted, of course, in significant differences in the characteristics of the spatial neutron flux distribution across the core and in the core-to-test-fuel neutronic coupling. With the exception of the M8 Calibration Experiment (M8CAL), which was performed in the upgraded core, all of the 
experiments discussed in this report were performed in the pre-upgrade core. A set of Inconel-clad fuel elements fabricated for use in a planned "TREAT Upgrade" core, which would have enabled TREAT to generate the harder neutron spectrum and higher fluence required for experiments using 37-pin test-fuel bundles within an exceptionally-large loop, was fabricated but never used in the reactor.

A key performance metric in TREAT experiments is the total energy deposition (TED) achieved in the test sample(s). The TED is related to TREAT core operation through a parameter called the power coupling factor (PCF). The PCF is the ratio of test sample power (or energy) per unit mass to the reactor total core power (or energy). It is typically expressed in units of W/g/MW or J/g/MJ. The PCF may change over the course of a transient, due to changes in core conditions including temperature, rod position, and the neutron flux reaching the test sample; this is captured in a parameter called the transient correction factor (TCF). Typically, the PCF and TCF were characterized for a particular experiment via a series of calibration runs performed prior to the experiment itself, in order to plan how the TREAT reactor power needed to be controlled during the experiment in order to achieve the desired energy deposition and temperature conditions in the tested sample.

\section{TREAT Experiment Program History}

During the 35-year period from 1959 to 1994, roughly 800 experiments were performed in TREAT to investigate the response of various nuclear fuels and fuel element designs to off-normal and accident-related transients, with and without the presence of water or sodium coolants. These experiments supported the evolving needs of U.S. civilian nuclear reactor development programs (LWR, LMFBR, and IFR). As those needs evolved, experiment requirements (which were driven by a need to ensure prototypic conditions for the test samples) spurred enhancements of TREAT's capabilities for performing increasingly complex experiments.

Most of the early experiments that have been conducted in TREAT were performed in rapid succession during the first several years of TREAT operation. They were designed to investigate fuel-water and fuel-sodium interactions for a wide range of fuel/cladding materials and fuel/coolant temperatures. Those experiments used small samples of unirradiated fuel in simple, mass-produced capsule-type containments heated using basic TREAT power transients (initially only bursts and later constant power "flattops") with application to both light-water-cooled reactors (LWRs) and sodium-cooled fast reactors (SFRs).

Starting in the mid-1960s, the experiment programs transitioned to predominantly address oxide fuels for liquid-metal-cooled fast breeder reactor (LMFBR) development, both for severe accident evaluations and for qualification of fuels for use in demonstration reactors. Further advances in TREAT's automatic control system allowed the production of power transients that could be tailored in shape, intensity, and duration. At the same time, the need arose and persisted to test larger fuel samples (longer pins and multi-pin bundles) under more-prototypic conditions, primarily to guide the development and validation of computer models and codes that describe offnormal and accident transient behaviors. This resulted in experiment designs (particularly ones that provided flowing coolant) that were more complex and had greater cost and schedule requirements. These more complex experiments could be performed at a rate of only about ten to fifteen experiments per year. Correspondingly, there was diminishing practical opportunity to investigate large parametric matrices of test conditions or to gain evidence of repeatability of 
observations. Each experiment thus became essentially unique, even though experiments continued to be planned and performed in "families" (sets, or series) within which key parametric changes were investigated.

The oxide-fuel LMFBR experimentation was pursued until the mid-1980s when attention was focused briefly on investigating the radiological source term during specific LWR accident scenarios, and also on investigating the behavior of modern metallic fuel for SFRs under severe transient overpower conditions. Experimental approaches, techniques, and equipment varied as needed during this time period in order to best address the variety of experiment objectives.

In general, TREAT experiments sought to create conditions that would reveal phenomena and demonstrate integral behavior of fuels and core materials that occur during reactor accidents, and to observe and measure those phenomena and behaviors. Topics of interest included:

- transient-induced fuel and cladding damage (composition and microstructural changes; and effects related to fission gas, chemical interactions, mechanical interactions, melting, and in-pin material vaporization),

- pre-failure fuel motions and coolant voiding,

- cladding failure thresholds,

- fuel-coolant interaction energetics,

- post-failure motions of fuel, cladding, and coolant,

- coolant channel blockages caused by disrupted fuel and cladding,

- $\quad$ and the complex inter-play among these behaviors.

The results of TREAT experiments were used for a variety of purposes, including:

- to help develop basic understanding,

- to acquire data relevant to developing empirical correlations which describe important behavior,

- to acquire data for validating mathematical models of certain phenomena, and

- to provide evidence useful in validating codes that describe complex integral behaviors such as might occur during the early stages of severe reactor accidents.

\section{Historical Experiment Series Included in This Report}

In the following sections of this report, an overview is given for each test series selected for inclusion in this edition of the report, using a common format for each overview. The format includes information in each of the following categories: the testing purpose, the approach taken, limitations involved in the approach, the in-pile experiment vehicle, the test fuel, imposed test conditions, results, and applications. Information is also provided on the power coupling determination, and references are indicated where the reader can find additional information.

More-detailed information is provided regarding each individual test by means of one-page summary descriptions grouped by experiment series in separate appendices at the end of the report. These one-page summaries provide an at-a-glance overview of each test. They include descriptions of the test sample(s), reactor operating conditions, measurements performed during and post-irradation, and a brief statement of the key results. These one-page summaries and the primary source materials 
used to compile them can also be found in TREXR [6]. (Note: one-page summaries in TREXR that are not included in this report should be considered preliminary drafts.)

The 19 test series for which detailed summaries have been developed and included in this edition of the report comprise a total of 90 tests. These tests and key parameter information regarding them are indicated in Table 4-1. (The series added since the publication of Edition 1 of this report are: D, E, EOS, F, H, J, R, RX, and S.)

Table 4-1. Key Characteristics of the Tests Addressed in This Report

\begin{tabular}{|c|c|c|c|c|c|c|c|c|}
\hline Test & Fuel material & Bond & Clad & Geometry & Coolant & $\begin{array}{l}\text { Imposed } \\
\text { coolant } \\
\text { flow }\end{array}$ & Overpower & $\begin{array}{c}\text { Fuel failure } \\
\text { disruption }\end{array}$ \\
\hline CDT1 & Irrad MOX & $\mathrm{He}$ & HT9 & 1-pin & Sodium & Steady & Slow & Minor \\
\hline CDT2 & Irrad MOX & $\mathrm{He}$ & HT9 & $1-$ pin $x 3$ & Sodium & Steady & Mod. Fast & Minor \\
\hline CDT3 & Irrad MOX & $\mathrm{He}$ & НT9 & $1-$ pin $x 3$ & Sodium & Steady & Mod. Fast & Moderate \\
\hline CO1 & Fresh MOX & $\mathrm{He}$ & SSTL & 1-pin & $\mathrm{NaK}$ & (none) & Fast & Major \\
\hline $\mathrm{CO} 2$ & Irrad MOX & $\mathrm{He}$ & SSTL & 1-pin & $\mathrm{NaK}$ & (none) & Fast & Major \\
\hline $\mathrm{CO} 3$ & Irrad MOX & $\mathrm{He}$ & SSTL & 1-pin & $\mathrm{NaK}$ & (none) & Fast & Major \\
\hline $\mathrm{CO} 4$ & Irrad MOX & $\mathrm{He}$ & SSTL & 1-pin & Sodium & Steady & Slow & Moderate \\
\hline CO5 & Irrad MOX & $\mathrm{He}$ & SSTL & 1-pin & Sodium & Steady & Slow & Minor \\
\hline C06R & Irrad MOX & $\mathrm{He}$ & SSTL & 1-pin & Sodium & Steady & Very fast & Major \\
\hline D1 & Fresh UO2 & $\mathrm{He}$ & SSTL & 7-pin & Steady & Steady & Steady & None \\
\hline D2 & Fresh UO2 & $\mathrm{He}$ & SSTL & 7-pin & Steady & Steady & Steady & None \\
\hline E1 & Fresh UO2 & $\mathrm{He}$ & SSTL & 1-pin & Sodium & Steady & Very fast & None \\
\hline E2 & Fresh UO2 & $\mathrm{He}$ & SSTL & 1-pin & Sodium & Steady & Very fast & Major \\
\hline E3 & Irrad U02 & $\mathrm{He}$ & SSTL & 3-pin & Sodium & Steady & Very fast & Major \\
\hline E4 & Fresh MOX & $\mathrm{He}$ & SSTL & 1-pin & Sodium & Steady & Very fast & Major \\
\hline E6 & Irrad MOX & $\mathrm{He}$ & SSTL & 1+6-pin & Sodium & Steady & Very fast & Major \\
\hline E7 & Irrad MOX & $\mathrm{He}$ & SSTL & 7-pin & Sodium & Steady & Very fast & Major \\
\hline E8 & Irrad MOX & $\mathrm{He}$ & SSTL & 7-pin & Sodium & Steady & Very fast & Major \\
\hline EBT1 & Irrad MOX & $\mathrm{He}$ & D9 & 1-pin & $\mathrm{NaK}$ & (none) & Mod. fast & Major \\
\hline EBT2 & Irrad MOX & $\mathrm{He}$ & D9 & 1-pin & $\mathrm{NaK}$ & (none) & Mod. fast & Major \\
\hline EBT3 & Irrad MOX & $\mathrm{He}$ & SSTL, D9, HT9 & $1-$ pin $x 3$ & Sodium & Steady & Mod. slow & None \\
\hline EBTB & Irrad MOX & $\mathrm{He}$ & SSTL & 1-pin & Sodium & Steady & Mod. fast & Major \\
\hline EOS1 & Fresh MOX & $\mathrm{He}$ & SSTL & 1-pin & (none) & (NA) & Very fast & Major \\
\hline EOS2 & Irrad MOX & $\mathrm{He}$ & SSTL & 1-pin & (none) & (NA) & Very fast & Major \\
\hline EOS3 & Irrad MOX & $\mathrm{He}$ & SSTL & 1-pin & (none) & (NA) & Very fast & Major \\
\hline $\mathrm{F} 1$ & Irrad MOX & $\mathrm{He}$ & SSTL & 1-pin & (none) & $(\mathrm{NA})$ & Steady & Major \\
\hline $\mathrm{F} 2$ & Irrad MOX & $\mathrm{He}$ & SSTL & 1-pin & (none) & (NA) & Very fast & Major \\
\hline F3 & Irrad MOX & $\mathrm{He}$ & SSTL & 1-pin & (none) & $(\mathrm{NA})$ & Steady & Major \\
\hline $\mathrm{F} 4$ & Irrad MOX & $\mathrm{He}$ & SSTL & 1-pin & (none) & (NA) & Steady & Major \\
\hline $\mathrm{F} 6$ & Irrad MOX & $\mathrm{He}$ & SSTL & 1-pin & (none) & $(\mathrm{NA})$ & Steady & Major \\
\hline H1 & Fresh MOX & $\mathrm{He}$ & SSTL & 1-pin & Sodium & Steady & Very fast & None \\
\hline $\mathrm{H} 2$ & Fresh MOX & $\mathrm{He}$ & SSTL & 1-pin & Sodium & Steady & Fast & Major \\
\hline
\end{tabular}




\begin{tabular}{|c|c|c|c|c|c|c|c|c|}
\hline H3 & Irrad MOX & $\mathrm{He}$ & SSTL & 1+6-pin & Sodium & Steady & Mod. fast & None \\
\hline $\mathrm{H} 4$ & Irrad MOX & $\mathrm{He}$ & SSTL & 1+6-pin & Sodium & Steady & Mod. fast & Major \\
\hline H5 & Irrad MOX & $\mathrm{He}$ & SSTL & 1+6-pin & Sodium & Steady & Mod. fast & Minor \\
\hline H6 & Irrad MOX & $\mathrm{He}$ & SSTL & 7-pin & Sodium & Steady & Mod. fast & Major \\
\hline $\mathrm{J} 1$ & Irrad MOX & $\mathrm{He}$ & SSTL & 7-pin & Sodium & Steady & Mod. Slow & Minor \\
\hline L1 & Fresh MOX & $\mathrm{He}$ & SSTL & 1-pin & Sodium & Steady & Slow & None \\
\hline L2 & Fresh MOX & $\mathrm{He}$ & SSTL & 7-pin & Sodium & Coastdown & Steady & Major \\
\hline L3 & Irrad MOX & $\mathrm{He}$ & SSTL & 7-pin & Sodium & Coastdown & Steady & Major \\
\hline L4 & Irrad MOX & $\mathrm{He}$ & SSTL & 7-pin & Sodium & Coastdown & Steady & Major \\
\hline L5 & Irrad MOX & $\mathrm{He}$ & SSTL & 7-pin & Sodium & Coastdown & Mod. fast & Major \\
\hline L6 & Irrad MOX & $\mathrm{He}$ & SSTL & 7-pin & Sodium & Coastdown & Mod. fast & Major \\
\hline L7 & Irrad MOX & $\mathrm{He}$ & SSTL & 3-pin & Sodium & Coastdown & Fast & Major \\
\hline L8 & Irrad MOX & $\mathrm{He}$ & SSTL & 3-pin & Sodium & Coastdown & Very fast & Major \\
\hline L01 & Fresh MOX & $\mathrm{He}$ & SSTL & 7-pin & Sodium & Steady & Fast & Major \\
\hline LO2 & Irrad MOX & $\mathrm{He}$ & SSTL & 7-pin & Sodium & Steady & Fast & Major \\
\hline LO3 & Irrad MOX & $\mathrm{He}$ & SSTL & 7-pin & Sodium & Steady & Slow & Major \\
\hline LO4 & Irrad MOX & $\mathrm{He}$ & SSTL & 7-pin & Sodium & Coastdown & Fast & Major \\
\hline LO5 & Irrad MOX & $\mathrm{He}$ & SSTL & 7-pin & Sodium & Low & Fast & Major \\
\hline L06 & Irrad MOX & $\mathrm{He}$ & SSTL & 7-pin & Sodium & Low & Fast & Major \\
\hline LO7 & Irrad MOX & $\mathrm{He}$ & SSTL & 7-pin & Sodium & Low & Very fast & Major \\
\hline M1 & Irrad U-5Fs & $\mathrm{Na}$ & SSTL & 1-pin & (none) & (NA) & Steady & Minor \\
\hline M2 & Irrad U-5Fs & $\mathrm{Na}$ & SSTL & 1-pin x 3 & Sodium & Steady & Mod. Slow & Minor \\
\hline M3 & Irrad U-5Fs & $\mathrm{Na}$ & SSTL & 1-pin x 3 & Sodium & Steady & Mod. Slow & None \\
\hline M4 & Irr + fresh U-5Fs & $\mathrm{Na}$ & SSTL & 1-pin x 3 & Sodium & Steady & Mod. Slow & Minor \\
\hline M5 & Irrad UPuZr & $\mathrm{Na}$ & D9 & 1-pin x 2 & Sodium & Steady & Mod. Slow & None \\
\hline M6 & Irrad UPuZr & $\mathrm{Na}$ & D9 & 1-pin x 2 & Sodium & Steady & Mod. Slow & Minor \\
\hline M7 & Irrad UPuZr, U-Zr & $\mathrm{Na}$ & D9, HT9 & 1-pin x 2 & Sodium & Steady & Mod. Slow & Minor \\
\hline PINEX2 & Irrad MOX & $\mathrm{He}$ & SSTL & 1-pin & $\mathrm{NaK}$ & (none) & Fast & None \\
\hline PINEX3 & Irrad MOX & $\mathrm{He}$ & SSTL & 1-pin & $\mathrm{NaK}$ & (none) & Mod. fast & Minor \\
\hline R3 & Fresh U02 & $\mathrm{He}$ & SSTL & 1-pin & Sodium & Coastdown & Steady & Major \\
\hline R4 & Fresh U02 & $\mathrm{He}$ & SSTL & 7-pin & Sodium & Coastdown & Steady & Major \\
\hline R5 & Fresh UO2 & $\mathrm{He}$ & SSTL & 7-pin & Sodium & Coastdown & Steady & Major \\
\hline R6 & Fresh UO2 & $\mathrm{He}$ & SSTL & 7-pin & Sodium & Coastdown & Steady & Major \\
\hline R7 & Fresh U02 & $\mathrm{He}$ & SSTL & 7-pin & Sodium & Coastdown & Steady & Major \\
\hline R8 & Fresh U02 & $\mathrm{He}$ & SSTL & 7-pin & Sodium & Coastdown & Steady & Major \\
\hline R9 & Fresh U02 & $\mathrm{He}$ & SSTL & 7-pin & Sodium & Steady & Mod. fast & Major \\
\hline R12 & Fresh U02 & $\mathrm{He}$ & SSTL & 7-pin & Sodium & Steady & Mod. fast & Major \\
\hline RFTL1 & Irrad MOX & $\mathrm{He}$ & SSTL & 1-pin x 3 & Sodium & Steady & Mod. fast & Minor \\
\hline RFTL2 & Irrad MOX & $\mathrm{He}$ & SSTL & 1-pin x 3 & Sodium & Steady & Slow & None \\
\hline RFTL3 & Irrad MOX & $\mathrm{He}$ & SSTL & 1-pin x 3 & Sodium & Steady & Slow & None \\
\hline RFTL4 & Irrad MOX & $\mathrm{He}$ & SSTL & 1-pin x 3 & Sodium & Steady & Fast & None \\
\hline $\mathrm{RX} 1$ & Fresh U02+SSTL & (NA) & none & cylinder & (none) & $(\mathrm{NA})$ & Steady & Major \\
\hline RX2 & Fresh U02+SSTL & (NA) & none & cylinder & (none) & (NA) & Steady & Major \\
\hline S2 & Fresh UO2 & $\mathrm{He}$ & SSTL & $5(+4)-$ pin & Sodium & None & Very fast & Minor \\
\hline
\end{tabular}




\begin{tabular}{|c|c|c|c|c|c|c|c|c|}
\hline S3 & Fresh U02 & $\mathrm{He}$ & SSTL & $5(+4)$-pin & Sodium & None & Very fast & Major \\
\hline S4 & Fresh UO2 & $\mathrm{He}$ & SSTL & $5(+4)-$ pin & Sodium & None & Very fast & Major \\
\hline S5 & Fresh U02 & $\mathrm{He}$ & SSTL & $5(+4)$-pin & Sodium & None & Very fast & Major \\
\hline S6 & Fresh U02 & $\mathrm{He}$ & SSTL & 7-pin & Sodium & None & Very fast & Minor \\
\hline S7 & Fresh U02 & $\mathrm{He}$ & SSTL & 7-pin & Sodium & None & Very fast & Major \\
\hline S8 & Fresh U02 & $\mathrm{He}$ & SSTL & 7-pin & Sodium & None & Very fast & Major \\
\hline S11 & Fresh U02 & $\mathrm{He}$ & SSTL & 1-pin & Sodium & None & Very fast & Major \\
\hline S12 & Fresh U02 & $\mathrm{He}$ & SSTL & 1-pin & Sodium & None & Very fast & Major \\
\hline STEP1 & Irrad U02 & $\mathrm{He}$ & Zircaloy-4 & 4-pin & Steam & Steady & Very slow & Major \\
\hline STEP2 & Irrad U02 & $\mathrm{He}$ & Zircaloy-4 & 4-pin & Steam & Steady & Very slow & Major \\
\hline STEP3 & Irrad U02 & $\mathrm{He}$ & Zircaloy-4 & 4-pin & Steam & Steady & Very slow & Major \\
\hline STEP4 & Irrad UO2 & $\mathrm{He}$ & Zircaloy-4 & 4-pin & Steam & Steady & Very slow & Major \\
\hline TS1 & Irrad MOX & $\mathrm{He}$ & SSTL & 1-pin & Sodium & Steady & Slow & Minor \\
\hline TS2 & Irrad MOX & $\mathrm{He}$ & SSTL & 1-pin & Sodium & Steady & Slow & Major \\
\hline NOTES: & Geometry & \multicolumn{7}{|c|}{$\begin{array}{l}\text { "1-pin," "3-pin," "4-pin," and "7-pin" refer to that number of identical pins in a single cluster } \\
\text { "1+6-pin" refers to } 1 \text { pre-irradiated pin surrounded by } 6 \text { fresh pins } \\
\text { "5 (+4)-pin" means } 5 \text { fuel pins and } 4 \text { hollow dummy pins interspersed in a cluster } \\
\text { "1-pin x 3" refers to } 3 \text { pins in separate flowtubes with common inlet and outlet flow headers }\end{array}$} \\
\hline & Overpower & \multicolumn{7}{|c|}{$\begin{array}{l}\text { Rate refers to the rise time to peak power. } \\
\text { Very fast }=0 \text { to } 0.3 \mathrm{~s} ; \quad \text { Fast }=0.3 \text { to } 1.5 \mathrm{~s} ; \quad \text { Moderately-fast }=1.5 \text { to } 3 \mathrm{~s} \text {; } \\
\text { Moderately-slow }=3-10 \mathrm{~s} \text { and includes } 10 \mathrm{\phi} / \mathrm{s} \text { simulations; } \\
\text { Slow }=10-60 \mathrm{~s} \text { and includes } 5 \mathrm{\phi} / \mathrm{s} \text { simulations; Very slow }>60 \mathrm{~s} \text {. }\end{array}$} \\
\hline & Fuel failure/disruption & \multicolumn{7}{|c|}{$\begin{array}{l}\text { Refers to severity of the damage to the fuel sample: } \\
\text { "None" means no cladding breach and at most minor fuel changes } \\
\text { "Minor" means significant fuel changes, and usually includes small breaching of cladding } \\
\text { "Major" means extensive cladding damage and gross redistribution of fuel sample materials }\end{array}$} \\
\hline
\end{tabular}

\section{CDT-series Experiments}

The CDT-series tested full-length, top-plenum, annular design (fuel, insulator, and reflector), irradiated MOX fuel pins in flowing sodium under fast and slow TOP (transient overpower) scenarios to show pre-failure fuel extrusion potential. A one-page summary of each CDT test is included in Appendix B.

\section{Number/Time}

The CDT-series consisted of three experiments performed in 1987 on fast-reactor FFTF-irradiated mixed-oxide fuel of the latest designs considered for deployment in the FFTF core. Five pins were exposed to severe overpower transients, comparing effects of solid-pellet versus annular-pellet designs at two power ramp rates. The results spanned the cladding failure thresholds and provided data useful for validating transient fuel-behavior models $[7,8,9,10]$.

\section{Purpose}

The three CDT tests were performed to provide failure threshold and pre-failure fuel-motion data for two designs of advanced FFTF-type mixed-oxide fuel pins, at two (medium and high) burnup levels, when exposed to different overpower transients $(5 \mathrm{~d} / \mathrm{s}$ and $1 \$ / \mathrm{s}$ reactivity insertion rates) relevant to FFTF severe accident safety assessment. The data were intended to be used for comparing the transient response of the two pin designs and evaluating the predictive capability of various fuel-pin 
transient performance codes and pin-failure models. The two pin designs differed by one having solid fuel and axial blanket pellets and the other having annular fuel and axial blanket pellets.

\section{Approach}

The tests were performed in a manner that would allow close comparison of the transient behaviors of the different fuel pins under conditions as prototypic of the simulated FFTF accident transients as feasible within the limitations of the TREAT reactor (e.g., utilizing the maximum-allowed TREAT energy). Conditions were intended to be created that would cause the fuel pins to marginally reach cladding failure. The set of test pins and test conditions was chosen to form a tight matrix of a few parameters in order to maximize the simplicity of making comparisons between the resulting behaviors. Thus, the same $5 \mathrm{\&} / \mathrm{s}$ reactivity insertion transient was used in two tests (CDT- 1 and CDT3 ), and solid-pellet designs and annular-pellet designs were tested at both overpower ramp rates. Furthermore, solid-pellet designs of both medium and high burnups were subjected to the same (1 $\$ / s$ ) transient, simultaneously with a pin of annular-pellet design. Sodium flow rates were chosen to achieve nominal FFTF conditions prior to the initiation of the power ramp.

\section{Limitations}

Although the transient behavior of an annular-pellet pin design was intended to be compared to the transient behavior of a solid-pellet pin design, the annular-pellet pins were of medium burnup whereas the solid-pellet pins were of high burnup. The fast fluence exposure of the cladding of the annular-pellet pins was correspondingly lower than that of the solid-pellet pins.

The accuracy of the empirical determination of the power coupling between the TREAT reactor core and the fuel in each of the pins tested was diminished by unexpected deficiencies in the test vehicles that were used, as noted below.

Because of the high temperature of molten oxide fuel, fuel expulsion from a failed pin into the coolant channel would tend to locally void the channel of sodium and quickly melt through the thin-walled flowtube. A breach in the flowtube where fuel is moving would affect the subsequent fuel motion dynamics.

\section{Experiment Vehicle}

Tests CDT-1 and CDT-3 were performed using two nominally-identical Single-Pin Test Loops (SPTLs). Test CDT-3 used a modified Mark-IIIC loop containing three fuel pins. Each pin was located within its own flowtube, with several thermocouples attached to the flowtube's outer surface. The flow rates through the three flowtubes in test CDT- 2 were nominally identical. In all three tests, each flowtube was initially surrounded by an argon-filled space to limit heat transfer radially outward from the flowtube.

It was discovered during posttest examinations of tests CDT- 1 and CDT- 3 that the expansion bellows near the top of each flowtube had failed. (It was unknown whether the failure had occurred before or during the test.). These failures allowed sodium to inadvertently enter and fill the spaces around the flowtubes, forcing out the gas that had been there, and contacting the flowtubes' outer surface and the attached thermocouples. The presence of the sodium subsequently affected heat transfer through the flowtube wall and measurement of the flowtube temperatures. This condition, if it had existed before the test, would have affected the interpretation and accuracy of the in-situ heat-balance measurements which were performed to determine the test-fuel-to-TREAT power coupling. Data intended to be obtained from the tests were limited also by premature failure of many of the flowtube thermocouples. 


\section{Test Fuel}

All five of the pins tested were of mixed-oxide fuel clad in HT-9 ferritic alloy, with $0.914 \mathrm{~m}$ fuel column height and a $6.5 \mathrm{~cm}$ axial blanket pellet stack at both ends of the fuel. Two pins (for CDT- 1 and CDT2) were of solid-pellet design of high-burnup (116-118 MWd/kg) and 17-18 x1022 n/ $\mathrm{cm}^{2}$ fast fluence. Two other pins (for CDT-2 and CDT-3) were of annular-pellet design (1.40 mm as-fabricated internal diameter) of medium burnup (63-65 MWd/kg) and 9.4-9.9 × $10^{22} \mathrm{n} / \mathrm{cm}^{2}$ fast fluence. The fifth pin (also for CDT-2) was similar to the annular-pellet pins except that it had solid pellets.

\section{Conditions}

CDT-1 and CDT-3 each subjected a single fuel pin to a power transient that increased on a 23-s efolding period, corresponding to a $5 \mathrm{q} / \mathrm{s}$ reactivity insertion rate in FFTF. In contrast, CDT- 2 subjected three fuel pins, each in its own flowtube, to a power transient that increased on a $0.67-\mathrm{s}$ e-folding period, corresponding to a $1 \$ /$ s reactivity insertion rate in FFTF. In each case, the power increase was continued until the cladding failure threshold would be reached or nearly reached. Leading into the overpower transient in each test was a 10-s hold at a level generating approximately nominal fuel power $\left(\mathrm{P}_{0}\right)$ for fuel of that burnup in FFTF (34-37 kW/m). In CDT-2, the power in the medium-burnup solid-pellet pin was about $14 \%$ higher than in the medium-burnup annular-pellet pin.

In CDT-1 and CDT-3, the unfortunate failure of the bellows near the top of the flowtubes diminished the ability to know the thermal conditions in the test fuel and in the sodium adjacent to the fuel pins. In CDT-2 the bellows remained intact, but the combination of temperatures indicated by the thermocouples, the sodium flowrate indicated by the flowmeter, and the assumed fuel pin power were mutually inconsistent, leading the analysts to conclude that the actual flow rate was $20 \%$ lower than indicated by the flowmeter.

\section{Results}

During the three tests, two of the five pins failed. Both of the medium-burnup pins (one solid-pellet and the other annular-pellet design) in fast-ramp test CDT- 2 withstood a peak power of about $17 \mathrm{P}_{\mathrm{o}}$ but breached just after peak power, as the power was rapidly dropping due to the reactor scram. The three other pins remained intact but apparently had been taken to near their failure thresholds. The high-burnup solid-pellet pin survived $4.0 \mathrm{P}_{\mathrm{o}}$ in slow-ramp test CDT- 1 and $17 \mathrm{P}_{\mathrm{o}}$ in test CDT-2. The medium-burnup annular-pellet pin in slow-ramp CDT-3 survived $4.5 \mathrm{P}_{\mathrm{o}}$ despite experiencing $20 \%$ more fuel enthalpy and $90^{\circ} \mathrm{C}$ higher peak coolant outlet temperature than did the high-burnup solidpellet pin in CDT-1.

Substantial axial, pre-failure, in-pin fuel motion occurred in all five pins, to the extent that the spring in the pin plenum was found to be fully compressed in each case - an upward movement of about 6 $\mathrm{cm}$.

Axial fuel motion in the solid-pellet pin in CDT- 1 began at a power of about $3.6 \mathrm{P}_{\mathrm{o}}$, when about $75 \%$ of the transient energy had been generated. About $6 \mathrm{~cm}$ length of molten fuel extruded into the region above the initial fuel column, lifting the upper axial blanket and plenum tube until the spring became fully compressed. In CDT-3 axial fuel motion in the annular-pellet pin began at about $3.8 \mathrm{P}_{\mathrm{o}}$. The fuel penetrated the annulus of both the upper and lower blankets along their entire lengths, and $\sim 10 \mathrm{~g}$ of fuel ( $5 \%$ of the fuel inventory) entered the plenum above the axial blanket. Posttest neutron radiography of CDT-3 showed a large axial variation of fuel density along the original fuel region. 
In CDT-2, in both the high-burnup and medium-burnup solid-pellet pins, axial fuel motion within the upper blanket region occurred at peak power, and by the end of the test, the upper blanket had been pushed up about $5 \mathrm{~cm}$ in the high-burnup pin and about $7.5 \mathrm{~cm}$ in the medium-burnup pin. The highburnup pin remained intact, but the medium-burnup pin failed just above the axial midplane, by meltthrough according to posttest computations. Posttest examination showed the failure extending over a region 7 to $25 \mathrm{~cm}$ above the midplane (i.e., from 0.58 to 0.77 relative fuel height). By the end of the CDT-2 test, molten fuel In the medium-burnup annular-pellet pin had reached and coated the inside of the spacer tube as high as $33 \mathrm{~cm}$ above the upper blanket column. The upper blanket was raised about $5 \mathrm{~cm}$ by axial fuel motion. As with the medium-burnup solid-pellet pin, the pin failed just above the axial midplane, by meltthrough according to posttest computations. The observed rapid loss of fuel just after peak power, and the fuel accumulation in a region centered in a region about 10 $\mathrm{cm}$ above the fuel midplane, was interpreted as being a consequence of midplane failure. Within 20 ms of cladding failure, molten fuel from that breached annular-pellet pin penetrated the flowtube in which that pin was located and spread into the gas-filled region beyond.

Posttest metallographical examinations indicated that in the solid-pellet pin in CDT- 1 the fuel areal melt fraction was $47 \%$ at $1 / 4$ height of the fuel column, $50 \%$ at midheight, and $56 \%$ at $3 / 4$ height. When compared with code computations, these values suggest that the actual fuel pin power was about $20 \%$ less than expected from pretest determinations. The melt fractions in that pin were notably lower than measured in the annular pin tested in CDT-3, which were $54 \%$ at $1 / 4$ fuel height, $75 \%$ at the midplane, and $68 \%$ at $3 / 4$ fuel height. The high-burnup solid-pellet pin that survived in the fastramp CDT- 2 test had measured melt fractions of $61 \%, 68 \%$, and $75 \%$ at $1 / 4$, half, and $3 / 4$ height levels, respectively.

The posttest measured peak cladding strain level in CDT-1 solid-pellet pin was 3.7\% (at the top of the initial fuel column and above, of which $2.7 \%$ occurred during the transient). In the cladding of the annular-pellet pin in CDT-3, however, there was only 1.4\% strain (all occurring during the transient). The surviving, high-burnup solid-pellet pin in CDT- 2 showed total strain of $\sim 7 \%$ near the midplane, $\sim 4 \%$ near the fuel top, and $\sim 10 \%$ about $5 \mathrm{~cm}$ above the fuel top.

\section{Applications}

Results from the three CDT transient overpower tests showed that the latest FFTF-design mixedoxide pins could survive to power levels greater than 4 Po during $5 \$ / s$ reactivity-ramp-rate accidents and up to 17 Po during $1 \$ /$ s accidents. The pins exhibited substantial pre-failure axial fuel movement, including through the center of annular blanket pellets, which could significantly introduce a negative reactivity effect on the accident. Significantly-lower transient-induced cladding strains in the annular-pellet design pins than in the solid-pellet design pins despite higher energy deposition in the annular-pellet pin, is attributable to the design difference. The test results were useful in evaluating transient fuel behavior models and the acceptability of the fuel designs for use in FFTF.

\section{Calibrations in TREAT}

Power coupling between the TREAT core and the test fuel was experimentally determined prior to the test transient by performing heat balance transients using the actual test fuel and test vehicle. The heat-balance transients were run at a steady, relatively-low power level representative of nominal pin power. 


\section{CO- and LO-series Experiments}

The LO-series tested 7 seven-pin bundles of full-length, bottom-plenum, annular, fresh and irradiated MOX fuel pins in flowing sodium under fast and slow TOP (transient overpower) and a range of TUCOP (transient undercooling-driven overpower) conditions. The CO-series tested single, fulllength, bottom-plenum, annular, fresh and irradiated MOX pins, 3 under fast TOP in stagnant NaK, and 2 under slow TOP and 1 under TUCOP in flowing sodium [10,11,12,13,14, 15, 16, 17]. A onepage summary of each CO-series test is included in Appendix C; one-page summaries of the LO-series tests are in Appendix D.

\section{Number/Time}

Six CO-series tests (C01 through C05, plus CO6R) and seven LO-series tests (L01 through L07) were performed during 1980-1983 within the UKAEA/USDOE PFR/TREAT Program.

\section{Purpose}

The LO- and CO-series tests were performed to investigate the timing and location of cladding failure and the pre-failure and post-failure fuel motions during overpower and loss-of-flow severe accident transients in large mixed-oxide-fueled fast reactors in which the fuel pins contain annular fuel and are grid-spaced and of bottom-plenum design and in which coolant voiding causes a positive reactivity addition resulting in a significant power burst. In conjunction, the tests were run to validate existing computational models of fuel pin failure and to strengthen the understanding of the relative roles of cladding-failure mechanisms.

\section{Approach}

The testing program consisted of tests on single pins (CO-series) and on seven-pin bundles (LOseries). The former provided azimuthal uniformity in fuel-pin thermal conditions, whereas the latter provided for effects of larger fuel mass and multi-pin geometry with multiple coolant channels. Tests C01 through $\mathrm{CO} 3$ were performed in stagnant NaK-filled capsules. The other tests were performed in flowing-sodium loops. Key parameters among the collection of tests included fuel burnup (medium and high), overpower ramp rate (slow and fast), and (for the loss-of-flow accident simulations) degree of coolant channel voiding when fuel was expelled into the channel (incipient voiding, partially voided, fully voided).

\section{Limitations}

The UK-design fuel differed from typical US-designed fuel in that the fission-gas plenum was below the fuel, and the pins were not wire-wrapped but were instead designed for use with grid spacers (which were used in the LO-series tests). Radial power depression in the fuel pins and within fuelpin bundles due to self-shielding of the test fuel within TREAT's thermal neutron spectrum was a significant factor in designing and planning the tests to provide maximum feasible prototypicality in radial temperature profiles through the pin. (For example, outer coolant channels in the pin-bundle tests LO3 through LO7 were enlarged to compensate for the higher fuel power near the bundle periphery.) Hydraulic characteristics of the test vehicle internals above and below the test fuel region were not fully prototypic of the above-core and below-core hydraulic situations in commercial plants and thus affected the prototypicality of the post-failure material motions. Melting of some of the flowtube after contact with fuel added molten steel mass to the dispersing molten fuel and cladding. TREAT energy capability allowed only about 20 full-power seconds of test-fuel energy generation in the TUCOP tests LO4-LO7. Conditions in static capsule tests were not considered relevant regarding application of post-failure fuel motions. 


\section{Experiment Vehicle}

Three different test vehicle designs were used: (a) NaK-filled single-pin capsules for C01, CO2, and CO3 (used to obtain early information) in which the fuel pin was thermally bonded by NaK to a massive nickel heat sink, (b) flowing-sodium single-pin loops for C04, C05, and CO6R (which provided greater thermal-hydraulic prototypicality than did the capsules), and (c) seven-pin-bundle flowing-sodium loops for LO1 through LO7 (which provided greater prototypicality regarding multipin and multi-channel effects during post-failure interactions of fuel, cladding, and coolant). In the pin-bundle tests, the pins were separated by grid spacers. In L01-LO3 a thick upper captor grid, representative of a fuel-pin hold-down device in a full scale reactor, was located above the top of the pin bundles. In L04-L07, immediately above the tops of the fuel pins was an 18.3-cm-long bundle of seven cladding tubes, each tube containing a steel rod equal in diameter to the fuel; this simulated the above-core structure of a full-scale reactor where molten material might freeze after pin failure and disruption.

\section{Test Fuel}

The fuel pins in all of the tests were of mixed U-Pu oxide fuel in stainless steel cladding. The MOX fuel pellet stacks in the pins were $91.4 \mathrm{~cm}$ high, with depleted urania breeder columns above and below the fuel. The fission gas plenum in the pin was located below the fuel column, separated from the fuel by a molybdenum knitmesh plug. Fresh (unirradiated) pins were used in C01, L01, and L06; pins of 9 at\% burnup were used in $\mathrm{CO} 3$ and CO5; the pins used in the other tests had $\sim 4$ at\% burnup. The $9 \%$ burnup fuel had been irradiated at a higher power level $(27.7 \mathrm{~kW} / \mathrm{m}$ burnup-averaged) than the $4 \%$ burnup fuel (17.1 kW/m burnup averaged), causing the fission gas retention to be about the same for both fuels.

\section{Conditions}

Fast ( 5 \$/s reactivity ramp simulation) transient overpower (TOP) conditions were applied in the three single-pin capsule tests (CO1, CO2, and $\mathrm{CO} 3$ on fuel of $0 \%, 4 \%$ and $9 \%$ burnup, respectively) and in the seven-pin loop tests (LO1 and LO2 on fuel of $0 \%$ and 4\% burnup, respectively). Slow (10 $\$ / s$ reactivity ramp simulation) overpower transients were applied in two of the single-pin loop tests (CO4 and CO5 on fuel of 4\% and 9\% burnup, respectively) and one seven-pin loop test (LO3 on fuel of $4 \%$ burnup). Simulations of transient undercooling-driven over-power ( TUCOP) conditions -caused by undercooling conditions and coolant voiding in a large fast reactor with positive sodiumvoid reactivity coefficient -- were generated in one single-pin loop test CO6R and four seven-pin loop tests L04 through LO7 (using 0\% burnup fuel in L06 and 4\% burnup fuel in C06R, L04, L05, and L07). The key variable in the TUCOP tests was the degree of voiding in the coolant channel at the time and location where fuel was initially expelled from the pin(s) - no voiding, some voiding, or complete voiding.

\section{Results}

In general, there was little or no prefailure fuel motion, only some axial expansion in some tests. Fresh fuel under fast TOP conditions failed near the fuel midplane. Over the burnup range tested (0 to $9 \%$ ), the power-to-fail under fast TOP $(\$ 5 / \mathrm{s})$ conditions was roughly 24 times nominal power and under slow TOP $(10 \$ / s)$ conditions was three times nominal power. Upon cladding failure, fuel motion was extensive and predominantly upward, in most tests dispersing to an extent corresponding to a negative reactivity addition of approximately 4 to $18 \%$ if it had occurred in a large fast reactor. (The worth analysis assumed a cosine-squared axial worth distribution with fuel-width at half maximum being $80 \%$ of the fuel height.) The exceptions were CO4 in which the worth decrease was about $30 \%$, and LO5 in which there was a 7\% worth decrease at pin failure but a final net worth increase of $4 \%$. Failed fuel tended to accumulate at the sites of the grid spacers, and its upward motion was strongly limited by the presence of the upper captor grid in the pin-bundle tests. In the TOP tests, vaporization 
of coolant created pressure that expelled fuel out both ends of the flow channel. In the TUCOP tests with preirradiated fuel (LO4, LO5, and LO7) upon pin failure an accumulation of fuel formed gradually at the failure site, grew axially, and then dispersed into regions above and below the original fuel zone. The fresh fuel in LO6 was less mobile. Upper and lower blockages (one of which was complete and the other either complete or nearly so) were found posttest in each of the seven-pin tests.

\section{Applications}

Combining models with the observed results provided good understanding of cladding failure mechanisms for fuel pins of the design tested, including internal pressurization, cladding melting, and swelling of hot solid fuel. Explanation of the initial fuel release from the pin was obtained from computations of the amount of molten fuel and existence or absence of a solid fuel crust at the time and location of failure. The observations were generally consistent with results of several computational models used to predict and analyze the tests performed with sodium flow.

\section{Calibrations in TREAT}

Power coupling between the TREAT core and the test fuel was experimentally determined for tests $\mathrm{CO} 1, \mathrm{CO} 2$, and $\mathrm{CO} 3$ by means of irradiating and radioactivity analysis of a fresh fuel pin (designated as the US/UK CAL activity) as well as by means of pre-test reduced-power heat-balance transients of the test pin within the test capsule. Power coupling determinations for $\mathrm{CO} 4$ and $\mathrm{CO} 5$ were made by TREAT irradiation of a fresh fuel pin and monitor wires in the activities designated as CO4-CAL, and similarly for CO6R by activities designated as C06-CAL. Similar activities were conducted for LO1 and LO2 (LO1-CAL), for LO3 (LO3-CAL), and for L04-L07 (LO4-CAL). In addition, heat-balance transients were typically run using the actual test fuel and vehicle prior to the final transient for each test.

\section{M-series Experiments}

The M-series tests focused on in-pin fuel motion in pre-irradiated metallic fuel pins (of circa 1980 metallic fuel design) during severe transient overpower, with cladding failure by rapid fuel-cladding metallurgical interaction [10,18, 19]. A one-page summary of each M-series test is included in Appendix E.

\section{Number/Time}

Seven M-series tests (M1 through M7) were performed during 1985-1987 in the pre-upgraded core. In anticipation of future tests in the series, neutronic calibration irradiations (M8CAL) were performed in the subsequently upgraded core.

\section{Purpose}

The M-series tests were performed to investigate in-pin motion of the latest designs of sodiumbonded fast-reactor metallic fuel during severe transient overpower accidents. Of particular interest was the potential for substantial pre-failure fuel motion ("extrusion") as a potential means of early negative reactivity insertion during an overpower accident. Another objective was to determine the nature of transient-induced cladding failure by the combined action of fuel-cladding metallurgical interaction and fission gas pressure. Evaluation of effects of fuel pin plenum pressure, retained fission gas, and sodium-logging of the fuel were of primary interest. The study of post-failure, in-channel fuel motion of the metallic fuel was not a major objective.

\section{Approach}

Test M1 heated two open-ended segments of an irradiated fuel pin at constant power in a dry-capsule environment (no coolant) and relied on high-speed photography to observe fuel motion out of the cladding. The following information refers to tests M2 through M7. Two or three intact fuel pins were 
heated with an exponentially-increasing power transient in a flowing-sodium loop. A total of 15 fuel pins were tested. Each pin was tested in a separate coolant environment so that more than one pin could be tested simultaneously, with a different flow rate past each pin, to permit different power/flow ratios between pins. The fuel pins were separated far enough to essentially eliminate neutronic effects of one pin upon the other. Fuel burnups ranged from zero to nearly 10 at\%. A main objective was to preserve, for posttest examination, the fuel conditions just before, and just after, cladding failure. It was also an objective to limit post-failure fuel motion so that the sodium loop would be able to be reused in subsequent tests.

\section{Limitations}

The availability of irradiated metallic fuel for TREAT testing was limited at the time. U-Fs fuel was used in M1 through M4 as a substitute for U-Pu-Zr fuel. Fuel in D9 cladding was used as a substitute for fuel in reference HT9 cladding. Inherent limitations of the TREAT reactor transient energy led to use of an 8-second exponential period in the power transient to ensure the heating rate became high enough to cause rapid fuel-cladding metallurgical interaction (at about $1350 \mathrm{~K}$ interface temperature) to be a major (or dominant) contributor to cladding failure (assisted by plenum pressure). This heating rate was higher than prototypic for unprotected transient overpower accidents in sodium-cooled fast reactor designs of interest at that time.

\section{Experiment Vehicle}

Test M1 used a dry capsule of a type that had been used in previous tests. Laser illumination of the test samples was employed, with high-speed cameras recording the fuel response to the power transient. Tests M2 through M7 used the Mark-III sodium loop, with fuel holders designed for three pins in separate sodium flow tubes (M2, M3, and M4) or two pins in separate flow tubes (M5, M6, and M7). Orifices in each flow tube were sized such that the flow rate past each pin would achieve the desired heating objectives for each pin.

\section{Test Fuel}

Tests M1 through M4 used irradiated 316 SS-clad U-5Fs fuel of burnups3.5 at\% in M1 and of burnups ranging from 0.3 to 7.9 at\% in M2 through M4. Tests M5 through M7 tested five D9-clad U-19Pu-10Zr fuel pins of burnup ranging from zero to 9.8 at $\%$ and one HT9-clad U-10Zr fuel pin of 2.9 at $\%$ burnup. All irradiations had been done in EBR-II. Active fuel columns were nominally $35 \mathrm{~cm}$ high.

\section{Conditions}

Test M1 applied steady power to the sample; no flowing coolant was present. Tests M2 through M7 brought power up to approximately nominal fast-reactor fuel power and then increased the power on an 8-second exponential period. The power was abruptly dropped at incipient cladding failure or immediately after cladding failure. Flowing sodium coolant was used during these six tests.

\section{Results}

Fuel extrusion was observed in all of the fuel pins tested, but varied substantially among the several fuel types. The low and medium burnup U-5Fs fuel extruded greatly prior to cladding failure, apparently because of the relatively-large amount of retained fission gas in that fuel. The high burnup U-Fs fuel and the U-19Pu-10Zr fuel extruded much less. Cladding failure occurred promptly when the fuel-cladding interface temperature reached approximately $1350 \mathrm{~K}$ in the U-5Fs and in the U-Pu-Zr fuel. Little fuel-cladding metallurgical interaction occurred in the U-Zr fuel pin despite reaching that range of interface temperature, apparently related to its higher fuel melting point. Initial post-failure fuel motion was consistently upward toward the failure site at the fuel-plenum interface, with ejected fuel being swept upward out of the original fuel zone. Material leaving the failure site was likely a 
molten alloy of fuel and cladding constituents due to the significant amount of fuel-cladding metallurgical interaction that preceded cladding breach.

\section{Applications}

Fuel extrusion modeling was aided by the wide range of fuel burnups and compositions tested, which provided insights into the relative roles of retained fission gas and in-fuel logged sodium. The 1350 $\mathrm{K}$ criterion for rapid penetration of cladding by fuel-cladding metallurgical interaction was confirmed by the results, although the reason for the lagging interaction in the U-Zr fuel was unclear. The few percent pre-failure extrusion of fuel (beyond thermal expansion) would cause a substantial negative reactivity effect in a large reactor undergoing an unprotected overpower transient.

\section{Calibrations in TREAT}

Experimental determination of the power coupling between the test fuel and the TREAT core was determined by irradiations in TREAT of fresh fuel pins and fissile monitor wires, which were then radiochemically analyzed. Such calibration activities were performed for test Mi (designated MI-CAL), for tests M2, M3, and M4 (designated M2-CAL and M4-CAL), and for M5, M6, and M7 (designated M7CAL). Later, an unusually-extensive set of neutronic calibration irradiations (designated M8-CAL) was performed to compare the neutronic coupling differences between the then-newly upgraded TREAT core and the pre-upgrade core.

\section{TS-series Experiments}

The TS-series tests investigated the prefailure axial fuel extrusion and the time and location of cladding failure in solid-pellet FFTF-type preirradiated MOX fuel pins during a slow (5 \$/s) TOP [9, $10,20,21]$. A one-page summary of each TS-series test is included in Appendix F.

\section{Number/Time}

Two TS-series tests (TS-1 and TS-2) were performed during 1983-84.

\section{Purpose}

The tests were performed to determine the time and location of failure of FFTF-type mixed-oxide (MOX) fuel pins during a slow (5 \$/s) overpower transient.

\section{Approach}

In both tests, a single pin of FFTF design and pre-irradiated in FFTF was tested in flowing sodium. Heating of the test fuel was programmed to match the heating that would occur in that type of fuel if such an accident were to happen in FFTF. The time of cladding failure was determined by sudden changes in flow rate due to vaporization of sodium by hot fuel expelled from the cladding. The location of failure was determined by the fast neutron hodoscope and thermocouples located axially along the wall of the flow tube surrounding the fuel pin.

\section{Limitations}

The only significant non-prototypicality apparently was the effect of the thermal neutron spectrum in TREAT resulting in a test-fuel radial power profile that was strongly peaked at the periphery of the fuel, unlike in a fast spectrum core such as FFTF. (This effect is typical of experiments in TREAT.) Postfailure fuel motion characteristics, which were not among the main objectives to determine, were affected by the prompt post-failure penetration of the flowtube, with molten fuel and cladding thereafter accessing additional space beyond that barrier. 


\section{Experiment Vehicle}

Both tests were performed in Single-Pin Test Loops (SPTL-Type B) designed specifically for fulllength, top-plenum fuel pins, such as FFTF driver pins. In flowing sodium. The loops were instrumented with flow, pressure, and temperature sensors, as well as acoustic detectors. The loop occupied the space of two TREAT fuel assemblies. A thin-walled flowtube, instrumented with many thermocouples attached to its outer surface, surrounded the test fuel pin and provided the boundary for sodium flow adjacent to the pin. Surrounding the flowtube was an alumina sleeve that served as a barrier to further penetration by molten fuel-pin materials after cladding failure. An inert gas space was between the flowtube and alumina sleeve. Dysprosium neutron filters located near the top and bottom of the test fuel were included to shape the axial power distribution in the fuel so as to reasonably match the axial power profile in FFTF.

\section{Test Fuel}

In both tests, the pin was a mixed-oxide (MOX) pin of FFTF-prototypic design $(91.4 \mathrm{~cm}$ fuel length; $20 \%$ CW type 316 stainless steel cladding of $5.84 \mathrm{~mm}$ OD, $0.38 \mathrm{~mm}$ wall thickness, wire wrapped, solid dished pellets of $4.94 \mathrm{~mm}$ diameter and $\sim 85 \%$ TD smear density, two uranium-dioxide solid pellets above and below the fuel, and a nickel reflector rod above the upper insulator pellets, with a spring holddown in the upper gas plenum). The fuel pin in TS- 1 had been pre-irradiated in FFTF to a burnup of approximately $2 \mathrm{MWd} / \mathrm{kg}$ at $41 \mathrm{~kW} / \mathrm{m}$ peak linear power. The pin in TS- 2 had been FFTFirradiated to $\sim 6 \mathrm{MWd} / \mathrm{kg}$ with a linear power that was $36 \mathrm{~kW} / \mathrm{m}$ at the peak burnup. Based on examination of a sibling pin from the FFTF irradiation, the test pin fuel had a central void of diameter $0.5 \mathrm{~mm}$ at the fuel midheight and $\sim 0.2 \mathrm{~mm}$ near the ends.

\section{Conditions}

To generate test-fuel temperature versus time at a rate as representative as during a $5 \mathrm{\$} / \mathrm{s}(\sim 21 \mathrm{~s}$ period) FFTF overpower transient as possible, the TREAT power history was programmed to account for the changing TREAT-to-test fuel power coupling during the transient, and the sodium flow rate was adjusted upward by $10 \%$. The reactor was programmed to scram immediately upon indication that the inlet sodlum flow rate in the test vehicle dropped by $50 \%$. The initial part of the TREAT power history (before the $5 \mathrm{~d} / \mathrm{s}$ period began) provided about $10 \mathrm{~s}$ of near-nominal power to preheat the test fuel.

\section{Results}

In TS-1, cladding failure (and immediate reactor scram) occurred $\sim 22 \mathrm{~s}$ into the power transient, at a test-fuel power 3.1 times the nominal $41 \mathrm{~kW} / \mathrm{m}$ power. About $1.7 \mathrm{~cm}$ of axial extrusion of molten fuel (approximately the free travel length allowed by the plenum spring) occurred approximately $5 \mathrm{~s}$ before failure. In TS-2, cladding failure occurred $\sim 24 \mathrm{~s}$ into the overpower ramp, at a test-fuel power 3.4 times its end-of-life level of $36 \mathrm{~kW} / \mathrm{m}$, preceded approximately $4 \mathrm{~s}$ earlier by $\sim 2.5 \mathrm{~cm}$ of axial fuel extrusion. In both tests, the cladding failed in the upper half of the fuel column (X/L $=0.84$ in TS-1) and rapidly penetrated the flowtube, allowing for more space into which the molten fuel-pin material and sodium could move.

\section{Applications}

The tests demonstrated that at low and medium burnups, FFTF-design MOX fuel pins can withstand a slow transient overpower event up to more than three times nominal power before cladding failure. They also showed the existence of significant pre-failure axial extrusion of molten fuel, which event was important (according to posttest analyses) in prolonging the time of failure during the tests. 


\section{Calibrations in TREAT}

Neutronic/radiochemical and thermal-hydraulic calibrations, referred to as TS-CAL, were performed to determine the power coupling between reactor and test fuel as a function of time during the specific transient planned for the experiments. This information was collected from irradiations of monitor wires, a fresh fuel pin, and preliminary lower-power irradiations of the test fuel pin in the SPTL with and without sodium flow.

\section{EBT-series Experiments}

The EBT-series investigated and compared the response of short, fast-reactor, irradiated MOX fuel and blanket pins, with various claddings, to unprotected overpower transients up to cladding failure $[9,22,23]$. A one-page summary of each TS-series test is included in Appendix G.

\section{Number/Time}

Four tests were performed in the EBT series (assuming EBTB was considered part of the EBT series), all during the 1983-84 time period.

\section{Purpose}

Three of the tests (EBT-1, EBT-2, and EBT-3) were performed to test irradiated mixed-oxide (MOX) fast-reactor pins under unprotected transient overpower (TOP) conditions of $10 \mathrm{c} / \mathrm{s}$ or $50 \mathrm{c} / \mathrm{s}$ reactivity ramp rate (relative to the FFTF reactor) to show how the transient response of the fuel pin differs in relation to the fuel's cladding material (316 SS, D9, or HT9). The fourth test (EBTB) investigated the response of a 316SS-clad MOX blanket fuel pin when subjected to a similar unprotected TOP.

\section{Approach}

EBT-1 and EBT-2 were single-pin tests in static NaK designed to directly compare the transient response of D9-clad irradiated MOX fuel with the results of a prior TOP test (in the HUT series) on 316SS-clad MOX. All three of those tests were performed with the same test vehicle and generated virtually identical thermal conditions in each pin up to cladding failure . EBT-1 used a high-burnup pin, and EBT-2 used a medium-burnup pin. (The HUT-series test was initially designated HUT5-1B and later renamed to HUT5-1B; in the TREXR database it is referred to as HUT5-1648, reflecting its TREAT transient number.) Test EBT-3 simultaneously tested three pins, each having a different cladding material (316 SS, D9, or HT9) in flowing sodium. The EBTB test with the blanket pin was also a single-pin test in flowing sodium.

\section{Limitations}

EBT-1 and EBT-2 were run in static NaK in order to be direct counterparts to the prior companion test (which tested a 316 SS-clad pin in static $\mathrm{NaK}$ ) although, because the coolant was static and not flowing, some loss of prototypicality in the pin's temperature distribution during the test would result. The TREAT thermal neutron spectrum caused strong power peaking near the periphery of the fuel, which is typical in TREAT tests and not representative of a fast spectrum.

\section{Experiment Vehicle}

Tests EBT-1 and EBT-2 were performed in a vehicle of the same design as used in the previous HUTseries test, i.e., a static capsule with thermal neutron shielding in which the test fuel pin was NaK bonded to a nickel heat sink. EBT-3 and EBTB were performed in Mark-IIC sodium loops with axial neutron flux shaping. In EBT-3, each of the three pins were located inside of its own flow tube, with each flow tube thermally isolated from the other. 


\section{Test Fuel}

Solid-pellet MOX fuel pins were used in EBT-1, EBT-2, and EBT-3, and a MOX blanket pin was used in EBTB. All of the pins had been pre-irradiated in the fast spectrum of the EBR-II reactor. The D9-clad pins in EBT-1 and EBT-2 had been pre-irradiated to $9.4 \%$ and $4.2 \%$ burnup, respectively. The three pins in EBT-3 had been pre-irradiated to burnups approximately in the 8 to $9 \%$ range, with peak linear powers of approximately $37 \mathrm{~kW} / \mathrm{m}$ and peak fluence of $\sim 6 \times 1022 \mathrm{n} / \mathrm{cm}^{2}$. The $316 \mathrm{SS}$-clad blanket fuel pin in EBTB had been pre-irradiated to about 3\% burnup and a cladding fluence of $1 \times 1023 \mathrm{n} / \mathrm{cm}^{2}$.

\section{Conditions}

In all four tests, the power transient began with a constant-power plateau lasting for several seconds (in order to preheat the test fuel at roughly the nominal pre-irradiation power level) prior to the overpower portion of the transient. The overpower portion began immediately thereafter and rose at a rate corresponding to either a $50 \mathrm{\$} / \mathrm{s}$ (EBT-1, EBT-2, and EBTB) or 10 \&/s (EBT-3) FFTF-type reactor reactivity ramp insertion rate continuing until cladding failure, or incipient failure, occurred. The power was abruptly terminated by TREAT scram at a pre-determined time or upon indication of test-pin cladding failure. Thermal conditions in the test pins were affected by heat transfer to the static NaK in EBT-1 and EBT-2 and by the flowing sodium in EBT-3 and EBTB.

\section{Results}

The cladding of the pins in EBT-1, EBT-2, and EBTB failed at power levels several times their nominal power (five times nominal power in EBT-1 and EBT-2 and seven times nominal power in EBTB), followed by extensive motion of molten fuel, gross disruption of the fuel (or blanket) pin with extensive relocation of molten fuel into the coolant channel. On the other hand, none of the three pins in EBT-3 failed; their peak powers had reached the range 2.4 to 2.9 times their steady-state irradiation power. In EBT-1, the cladding of the high-burnup pin first breached near the top of the fuel column (at a power about 7\% lower than in the corresponding HUT test), followed by significant fuel expulsion to above the test fuel region and significant voiding of fuel along the length of the fuel column. In EBT-2, the cladding of the medium-burnup pin first breached near the fuel midplane (at a power about 18\% lower than in the HUT test), followed first by fuel loss from the top and bottom of the fuel column and then fuel movement from the midplane to the bottom of the fuel column. The blanket pin in EBTB apparently failed (at 7 times nominal power) first near the fuel midplane, with ejected molten fuel then being swept up to the top of the fuel column where it accumulated and blocked the coolant flow channel. The three unfailed pins in EBT-3 experienced significant (about $36 \%$ areal fraction) fuel melting but little or no transient-induced cladding strain.

\section{Applications}

The tests demonstrated the ability of the fuel pins to withstand the imposed power transients up to several times their nominal steady-state irradiation power. Data from the tests were useful in evaluating the predictive capability of fuel-pin behavior codes and to help support the safety basis for irradiations of advanced fuel designs in FFTF.

\section{Calibrations in TREAT}

For EBT-1, EBT-2, and EBT-3, the power coupling between the test fuel and the TREAT core was measured before each test by heating the test fuel, inside the test vehicle, using a relatively-low-level, steady power transient, measuring the resulting temperature rise of the coolant and surrounding structure, and computing the fuel power needed to produce the measured thermal effects. No separate TREAT irradiations of additional pins were involved in these calibration activities. For EBTB, a separate calibration irradiation in TREAT (EBTB-CAL), using a neutronic mockup of the EBTB test vehicle, was performed on a fresh blanket fuel pin of design similar to the EBTB test pin and also on 
flux monitor wires, followed by radiochemical analysis of those items to determine fission density and axial profile, thus yielding the desired power coupling information for the EBTB test.

\section{L-series Experiments}

The L-series tests demonstrated the fuel-motion responses of pre-irradiated fast-reactor-type MOX fuel pins to several different loss-of-flow accident conditions including strong overpower ramps [24, $25,26,27,28,29,30,31,32,33][34,35,36,37]$. A one-page summary of each L-series test is included in Appendix H.

\section{Number/Time}

Eight L-series experiments were performed during the time period 1970 - 1978.

\section{Purpose}

The tests were performed to measure the timing and magnitude of spatial redistributions of fuel during simulations of various aspects of a fast-reactor hypothetical loss-of-flow (LOF) accident. Behaviors of fuel of various types, under various imposed conditions, pertinent to a range of subassembly types (power level, burnup, power level) would be demonstrated in order to indicate how each type might respond to, and subsequently affect, the course of the accident. The results were to be useful in validating analyses of the loss-of-flow accident for a MOX-fueled CRBR fast reactor.

\section{Approach}

Because this test series was the first to simulate loss-of-flow conditions in TREAT experiments in sodium, and because of limited availability of pre-irradiated fuel of the types preferred (particularly prototypic-length fast-flux-irradiated pins of FFTF/CRBR-type fuel pins), and because of thenexisting limitations of the TREAT control system, it was necessary for the prototypicality of the simulations to progress from test to test as experimental capabilities and fuel availability improved with time. The initial tests would need to utilize constant-power "flattop" transients, whereas shaped transients (e.g., flattops concluded with a programmed reactivity ramp to simulate a LOF-driven overpower situation) would be used when the TREAT control program was enhanced to produce them. Constant coolant flow during tests would be used until the sodium loop system became capable of providing programmed coolant flow coastdowns. Short-fuel-length irradiated pins (representing the top of the fuel of full-length FFTF-type pins) would be used until longer fuel pins became irradiated, and the longer pins would be used despite being irradiated in a thermal flux because no fast-flux-irradiated long-fuel pins would become irradiated until much later. Single pins and small bundles of pins, with pins of various burnups and pre-irradiation fuel restructuring, would be used depending upon which type of reactor subassembly would be the represented in the particular test. Reactivity ramps would be included in the test if the test was intended to simulate fuel in lower-power subassemblies responding to a power transient caused by reactivity effects in the lead assemblies of a reactor.

\section{Limitations}

Aspects of the approach taken in conducting this series of tests were chosen because of the existing limitations in availability of fuel for testing and capabilities of testing facilities. These were described above. Additional limitations needed to be taken into account: some in test planning and some in posttest analyses. One was the size of the fuel sample (one, three, or seven pins), limited by safety reasons or fuel availability, which would affect how well the test sample would represent a muchlarger array of pins in a reactor subassembly. Another was the ability to design a test environment of the pins such that the pins could be adequately heated to high temperatures in initially-flowing sodium with a representative fuel-to-sodium mass ratio, and with adequate instrumentation to 
monitor the thermal conditions of the fuel, and simultaneously with adequate fuel containment during the meltdown. The loop test section designs that were used provided for good conditions to the point of fuel dispersal. After being contacted by hot fuel, however, the steel flow tube surrounding the fuel pins readily melted and breached, opening up new lateral spaces for molten fuel, steel, and sodium to flow into, and contributing to the amount of molten steel in the meltdown mass. Experience with the sodium loop was still being developed, and problems with the loop flowmeters were initially encountered that, in some tests, significantly reduced the amount of information obtained.

\section{Experiment Vehicle}

Mark-II integral sodium loops were used in all of these tests. The loops provided the flowing sodium and the thermal and pressure containment of the test fuel. Thermal-neutron-absorbing layers (flux "shaping collars") were located around the loop periphery to provide a suitable axial power profile in the test fuel. Inside the loop test section was the test train that held the test fuel sample and provided the sodium flow area and flow perimeter to best represent conditions in a large assembly of pins. Test L1 used a single-pin test train. The flow perimeter in the seven-pin-bundle tests (L2, L3, and L4) and the three-pin-bundle tests (L5 through L8) was a fluted stainless steel tube surrounded by an inert-gas space. The flutes in the tube were geometric representations of adjacent fuel pins in a larger array.

\section{Test Fuel}

All of the tests used FFTF-type mixed-oxide fuel pins but with fuel regions shorter than standard FFTF fuel pins. The pins in L1 and L2 were not pre-irradiated. Tests L3 and L4 used fast-spectrumirradiated, medium-burnup pins; in L3 the fuel had been low-power irradiated, whereas in L4 the fuel had been high-power-irradiated. Fuel pins in tests L1 through L4 had fuel columns $34.3 \mathrm{~cm}$ high (the height of the EBR-II core in which the pins in L3 and L4 had been pre-irradiated). Pins for tests L5 through L8 were all pre-irradiated in the thermal flux of the GETR reactor and had fuel column heights of $86.4 \mathrm{~cm}$, only $5 \%$ less than the FFTF core height. For L5 through L7, the pre-irradiation of all of the fuel pins resulted in moderate-power fuel structure; their burnup was 8\% for L5 and 3\% burnup for L6 and L7. Test L8 used pins like those in L6 and L7 except for having been irradiated at low power.

\section{Conditions}

Without a capability for shaped power transients or coolant flow coastdown at the beginning of this series, and being an initial (therefore exploratory) LOF test, test L1 subjected only a single, unirradiated pin to a rough simulation of a LOF condition by exposing the pin to a succession of steady-power runs, each with a lower coolant flow rate than the previous run, with the intent of closely approaching, but not exceeding, the cladding failure threshold. Test L2 included the improvements of testing a seven-pin bundle with a coolant coastdown, still using fresh fuel and a constant-power run with flow coastdown, with conditions exceeding cladding failure. Flow coastdowns were used in all of the subsequent tests. Tests L3 and L4 added the feature of using fastspectrum pre-irradiated fuel pins (with $34.4 \mathrm{~cm}$ fuel height as in L1 and L2), again in seven-pin bundles and with a constant-power run in each test. Tests L5 through L8 also used pre-irradiated fuel, but now with $84.4 \mathrm{~cm}$ fuel height (although with thermal-spectrum pre-irradiation), and significantly ramped up the power after a few seconds at steady power. In L5 the peak power reached about six times nominal, in L6 about ten times nominal, in L7 about 23 times nominal, and in L8 about 75 times nominal. The strong overpower parts of the transient in the last four tests all generated about the same amount of TREAT energy and thus (if the power coupling did not change as the test fuel dispersed) about the same amount of test fuel energy. In each test, an objective of avoiding the generation of significant fuel vapor pressure was achieved. 


\section{Results}

In L1, unexpected sodium boiling apparently occurred, possibly under the spacer wire wrap of the pin and the adjacent in-channel thermocouple, causing severe flow anomalies and probably strong azimuthal and axial temperature gradients in the pin. The pin remained intact but was highly distorted. In contrast, the seven fuel pins in test L2 (the first test performed in TREAT in which a fuel pin was destroyed by exposure to loss-of-flow conditions) were highly disrupted, with gross movement of molten fuel and steel and formation of upper and lower flow blockages that posttest appeared to be complete. Gross disruption of the original fuel region and accumulation of molten steel and fuel near the top and bottom of the original fuel region likewise characterized all of the subsequent tests. Generally much of the flowtube adjacent to the fuel regions was also melted, thus contributing to the overall molten fuel mass and allowing molten material to flow into the surrounding gas-filled space. Details of fuel motion were provided by the fast neutron hodoscope and varied considerably from test to test (and thus probably also did the steel motion). Final fuel and steel configurations also varied in their details, but generally little mixing of the two materials was found posttest.

\section{Applications}

The fuel motions observed in the later tests in the series were evaluated in terms of the reactivity changes they would produce in a large fast reactor such as CRBR. For this purpose, selected CRBR fuel worth axial distributions were used. This provided a means by which the results of accident analysis models and codes could be compared to the test results. The SAS accident analysis code was developed through its various editions at the time (SAS1A, SAS2A, SAS2B, SAS3A, and SAS3D) in parallel with the L-series tests as the two programs collaborated with and assisted each other. A combination of the predictive analyses and empirical results from the test provided a basis for the development of scenarios describing the sequence of key LOF events in each test.

\section{Calibrations in TREAT}

Typically the final transient in each test was preceded by a heat-balance transient at a reduced, steady power and nominal flow rate in order to experimentally verify the power coupling between the test fuel sample and the TREAT core. The later tests in the series involved neutronic calibrations performed by TREAT-irradiation and radiochemical analysis of a fresh fuel pin representing the pins to be transient tested, thereby determining the absolute power coupling between the fuel and TREAT core. Pellets from the calibration pins were also core-drilled to obtain samples for radiochemical analysis to determine radial fission profiles.

\section{PINEX-series Experiments}

The PINEX tests investigated the potential for in-pin axial fuel motion in special, annular-design lowburnup MOX fuel pins undergoing $3 \$ / \mathrm{s}$ and 50 \&/s unprotected reactivity-ramp power excursions $[38,39]$. A one-page summary of each PINEX-series test is included in Appendix I.

\section{Number/Time}

Two PINEX-series tests, PINEX-2 and PINEX-3, were performed in 1978.

\section{Purpose}

The tests were performed to investigate the potential for in-pin pre-failure axial fuel motion to significantly mitigate severe hypothetical transient overpower accidents in mixed-oxide fueled fast reactors. A second purpose of the tests was to evaluate the capability of the pin-hole imaging system to monitor the motion of nuclear fuels in TREAT experiments. 


\section{Approach}

Fuel pins were designed with an axial hole through the fuel, through the two insulator pellets above the fuel, and through the reflector above the insulator pellets, in order to provide a pathway for molten fuel to escape from the fuel region prior to cladding failure. The timing and extent of axial fuel motion was to be determined in each of two tests that differed only in the rate of power rise during the overpower burst, i.e., one representing a $3 \$$ /s unprotected reactivity ramp in FFTF and the other representing a $50 \mathrm{~d} / \mathrm{s}$ ramp rate. The relationship between TREAT power and sample power was established such that the TREAT power level would rise high enough during the transient (above $1000 \mathrm{MW}$ ) to permit adequate signal strength to the pinhole (PINEX) fuel detection system that was to be evaluated.

\section{Limitations}

The thermal spectrum used in the pre-irradiation of the test fuel samples created a different radial distribution of fuel restructuring and fission-gas retention than a fast spectrum would have generated. In addition, the thermal spectrum in TREAT caused the effect (typical in TREAT tests) of depositing heat preferentially near the periphery of the test fuel sample. These effects affected the timing and progress of fuel melting and fission gas release in the test fuel pin that differed from what would have occurred in a fast spectrum. The effects would be expected to differ between the two tests due to the different power rise rates and the resultant different progression of the radial temperature profile during the overpower part of the transient. In addition, due to the static coolant, the axial temperature gradient in the fuel was not peaked toward the top of the fuel as it would have been in a flowing coolant environment, and this would have affected the axial progression of fuel melting above the midplane. The extent to which these non-prototypicalities affected the outcome of each of the tests either qualitatively or quantitatively was addressed by analyses.

\section{Experiment Vehicle}

Both tests used the same type of test vehicle designed to contain a single fuel pin in a static NaK coolant environment surrounded by a thick nickel heat sink. Thermal-neutron attenuation was provided by boron-containing layers around the outside of the vehicle. Heaters located axially adjacent to the test fuel provided an initial axial temperature profile. The vehicle was designed such that it would house the fuel pin during the pin's pre-irradiation in the General Electric Test Reactor (GETR) and also serve as the test vehicle during the TREAT transient test.

\section{Test Fuel}

Identical specially-designed mixed-oxide fuel pins ( $25 \mathrm{wt} \% \mathrm{Pu}, 75 \mathrm{wt} \% \mathrm{U}$ ) were used in both tests. The fuel pellets were annular, with $0.81 \mathrm{~mm}$ diameter fabricated central hole. The pellet stack was $86.4 \mathrm{~cm}$ high. Above the stack were two annular $\mathrm{UO}_{2}$ pellets having $1.78 \mathrm{~mm}$ ID. Above the insulator was a $49-\mathrm{mm}$ long nickel rod with $1.70 \mathrm{~mm}$ diameter central hole. The fuel pins were nominally of FFTF design, with Type 316 SS cladding ( $20 \%$ cold-worked) of $5.84 \mathrm{~mm}$ outer diameter and $0.38 \mathrm{~mm}$ thickness. The fuel pins were pre-irradiated in the GETR thermal-spectrum reactor to approximately 2 at $\%$ burnup.

\section{Conditions}

Each test was designed, within the limits of the TREAT reactor and the test vehicle that was used, to produce test fuel thermal conditions associated with unprotected reactivity ramp excursions in FFTF as closely as practical, specifically a $3 \$$ /s ramp in PINEX-2 and a $50 \mathrm{\$} / \mathrm{s}$ ramp in PINEX-3. At the beginning of each test, the fuel was preheated at nominal power for a few seconds, followed immediately by a power rise corresponding to the desired ramp rate. In each test, the TREAT reactor was programmed to scram at the predicted time of initial fuel melting, anticipating that the axial fuel motion that would begin at that time would, if it were to happen in the core of a fast power reactor, 
cause a negative reactivity insertion and power reversal. The stagnant NaK environment, compared to a flowing-coolant environment, of the pin was deemed to be an acceptable substitute in these tests. TREAT power during each transient rose above the $1000 \mathrm{MW}$ level needed for acceptable signal to the PINEX fuel detector system.

\section{Results}

The fuel pin transient behavior differed substantially between the two tests, presumably due to the difference in how the different heating rate (and heat loss rate to the coolant) caused the fuel melting progression into fission-gas-containing regions of the fuel. Fuel in PINEX-2 became free to move axially within the fuel central hole axially past the top of the fuel and into the insulator, reflector, and plenum. This apparently decreased the temperature and pressure loading on the cladding, and the cladding remained intact. In contrast, with the slower transient in PINEX-3 it appeared that the central void near the top of the fuel did not open to allow significant axial fuel movement through that area, and gross disruption of cladding and fuel resulted. The PINEX detector system provided fuel location information useful in the analysis of the test, supplementing the fuel-motion information provided by the TREAT hodoscope.

\section{Applications}

The ability of MOX fuel to flow axially, prior to pin failure, within annular-design fuel pins as used in these tests was demonstrated to be feasible, but dependent upon the particular heating conditions and retained fission gas distribution in the fuel. Results from the real-time fuel monitoring data and from detailed posttest analysis of the intact fuel pin from PINEX-2 yielded particularly valuable information for comparison with code predictions.

\section{Calibrations in TREAT}

Power coupling between the test fuel and TREAT core was experimentally determined by measurements taken during low-energy transient irradiations of the actual test fuel in the test vehicle. No separate irradiations of calibration fuel pins were made. (The PINEX-3A test was subsequently performed to explore the sensitivity of the pinhole-intensified imaging system to low fuel specific power levels.)

\section{RFT-series Experiments}

The RFT tests showed that FFTF driver fuel pins can withstand unprotected overpower transients well beyond the secondary PPS (Plant Protective System) limit and provided data for calibrating transient fuel behavior codes [10, 40, 41, 42, 43, 44]. A one-page summary for each RFT-series test is included in Appendix J.

\section{Number/Time}

There were four tests in the RFT series, performed between 1982 and 1984. In addition, a preliminary test RFT-CAL-L was performed to provide data on power calibration, loop thermal-hydraulics, and fuel pellet design impact on cladding strain.

\section{Purpose}

The objectives of the RFT series were: (1) to demonstrate the capability of FFTF reference driver fuel pins, irradiated in FFTF, to accommodate an FFTF secondary plant protective system (PPS) terminated overpower transient without damage, (2) to verify that a significant margin to failure exists by extending the transient overpower beyond the secondary PPS limit of 1.25 times normal rated power, and (3) to establish transient-induced measurable changes in the test pins as a basis for 
fuel pin performance code correlation, improvement, and validation. Responses of FFTF driver fuel pins of various burnups and irradiation power levels were evaluated for reactivity ramp rates of 5 \$/s, 50 \&/s, and $1 \$ / \mathrm{s}$.

The objectives of RFT-CAL-L were to provide data needed for pretest analysis and planning of the RFT tests, i.e., regarding power coupling between FFTF driver fuel pins and the TREAT core and regarding sodium loop thermal-hydraulics for calibrating the loop thermal-hydraulic model, and also to provide data on fuel pin transient performance for use in calibrating fuel pin transient performance codes.

\section{Approach}

In the RFT series, fuel pin responses to unprotected overpower transients would be tested and evaluated for several fuel burnup and irradiation-power levels and for several reactivity ramp rates. Test conditions were set to closely match code-predicted fuel and cladding temperatures during the transient, and to reach conditions involving substantial fuel melting, measurable cladding strain, but no cladding failure. To best match the thermal transient in the test fuel and cladding with the thermal transient analytically computed for the FFTF unprotected reactivity excursion, both the test-fuel power and the coolant flow rate were specified to be higher (generally 10-15\%) than the conditions which the pins experienced in FFTF; this adjustment helped to compensate for the depressed radial power profile in the test fuel due to the TREAT thermal neutron spectrum. The transients were to continue long enough to cause significant fuel melting and cladding strain but not to reach cladding failure conditions. Posttest examination of the pins would provide key data for comparison with analytical code predictions of fuel pin thermal-mechanical response.

\section{Limitations}

The thermal neutron spectrum in TREAT produces a large radial flux depression toward the center of test fuel samples, in contrast to the profile that is present in a fast spectrum. This effect on the fuel pin temperature history during the transient was reduced by adjusting the starting conditions of fuel temperature and power from their nominal FFTF conditions.

\section{Experiment Vehicle}

All of the tests in this series used Mark-III integral sodium loops containing a three-pin test train in which each pin is located in a separate flowtube. Instrumentation included flowmeters on the loops and numerous thermocouples on the test trains. Flux shaping collars on the loops were included to provide a suitable axial power profile in the test fuel.

\section{Test Fuel}

In the four RFT series tests, all of the fuel pins were FFTF driver fuel pins that had been irradiated in FFTF. In RFT-L1 and RFT-L2, the pins had been irradiated to very low (about $0.2 \%$ ) burnup, three pins at $26-29 \mathrm{~kW} / \mathrm{m}$ and three at $41 \mathrm{~kW} / \mathrm{m}$. The pins in RFT-L3 and RFT-L4 had been irradiated in FFTF at $35-38 \mathrm{~kW} / \mathrm{m}$ to two burnups $(2.6 \%$ and $\sim 5.3 \%)$. Both types of pins were exposed to a $5 \mathrm{c} / \mathrm{s}$ overpower transient (in RFT-L3) or to a 1 \$/s transient (RFT-L4).

In the RFT-CAL-L test, all three pins were unirradiated. Two were FFTF driver pins. The third was a specially-designed MOX fuel pin with fully enriched uranium and several fuel sections of various pellet inner and outer diameters and fuel density, and one section was made with a radial slot.

\section{Conditions}

In the RFT series, the $0.2 \%$ burnup pins of each irradiation power were subjected to a $50 \mathrm{q} / \mathrm{s}$ overpower in RFT-L1 reaching a peak power of about 5 times nominal; in RFT-L2 pins of that burnup were subjected to a $5 \mathrm{\$} / \mathrm{s}$ overpower reaching 3.4-3.7 times nominal in the lower-power-irradiated 
pins and 2 times nominal in the higher-power-irradiated pin. In RFT-L3 and RFT-L4, pins of both burnups were subjected to either a $5 \mathrm{q} / \mathrm{s}$ overpower reaching 1.8 times nominal or to a $1 \$ / \mathrm{s}$ overpower reaching about 6.8 times nominal.

In the RFT-CAL-L test, the fuel was initially subjected to a $1 \$ / \mathrm{s}$ overpower spike (to produce fuel melting and fuel-cladding interaction in the specially-designed fuel pin), and then was subjected to two lower-power transients to build up fissions needed for radiochemical analysis.

\section{Results}

All of the RFT tests produced substantial fuel melting, and measurable cladding strain occurred in many of the tests. All of the pins survived to power levels well beyond the secondary PPS limit. The failure threshold was exceeded in one of the pins (in RFT-L1). Detailed posttest characterization of the test pins was performed for use in comparison with analytical predictions.

In RFT-CAL-L, the special fuel pin underwent fuel melting and cladding strain, the latter being a strong function of the fuel design features (annular, fuel-cladding gap size, fuel density, presence of radial slot). Neutronic and thermal-hydraulic calibration data were obtained for use in preparing the RFT tests.

\section{Applications}

The test data were used for detailed comparison with code-calculated predictions of fuel melting and cladding strain.

\section{Calibrations in TREAT}

In addition to the RFT-CAL-L test, heat-balance transients at constant test-fuel power in TREAT were performed before each test to determine the power coupling between the test fuel and the TREAT core. During these transients, the test-fuel temperatures were kept below their prior irradiation temperatures in FFTF. In some cases, flux monitor wires were attached to the outside of one or more flowtubes for use in measuring the axial flux profile posttest.

\section{STEP-series Experiments}

The STEP-series tested 4 four-pin bundles of short, pre-irradiated LWR pins to failure in flowing steam and collected and characterized volatile fission products transported downstream [45]. A onepage summary of each STEP-series test is included in Appendix K.

\section{Number/Time}

Four Source-Term Experiments Project (STEP) tests STEP-1 through STEP-4 were conducted during 1984-1985 by an international consortium headed by EPRI and including Ontario Hydro of Canada, US Department of Energy, US Nuclear Regulatory Commission, and Belgonucleaire.

\section{Purpose}

The tests were performed (a) to characterize fission products and structural materials that may be released from light-water reactor (LWR) fuel during postulated risk-dominating pressurized-water reactor (PWR) and boiling-water reactor (BWR) severe accidents and transported downstream, and (b) to generate and collect data on associated fuel-rod heat-up, cladding oxidation, and failure. The intended characterization focused on the physicochemical properties of the biologically-important volatile fission products released early in such transients. 


\section{Approach}

In each test, pre-irradiated fuel pins were subjected to a flowing steam environment at pressures and temperatures which, at the time of release of volatile fission products and noble gases from the pins, would be consistent with a particular accident scenario. Various types and orientations of surfaces were provided downstream upon which fission products could collect during the tests. Deposited volatile fission products would be examined and characterized posttest.

\section{Limitations}

With the relatively-short test fuel, conditions were simulated for the time during the reactor accident scenarios when the water level has fallen below the axial mid-height of the fuel. The means of aerosol sample collection was limited by the available space within the vehicle. A small pin-bundle was used to represent full-size pin arrays, requiring a flow tube with high surface area per pin. Fuel heating rate in STEP-2 had to be higher than desired because of facility limitations.

\section{Experiment Vehicle}

A square-arrayed four-pin bundle in each test was surrounded by a high-density zirconia tube within which steam flowed past the fuel pins. The steam was generated by an ex-pile system. After the steam passed through the pin bundle and fission-product collectors it was received and condensed ex-pile. The sample collection tree included many coupons of a wide variety of materials. Some coupons were parallel, and some perpendicular, to the flow. Two aerosol canisters were mounted alongside the pin plenum region above the fuel columns. Each canister contained three chambers, each of which was opened for a predetermined time interval during the test. Each canister contained 14 sample collection stages, each of which contained fine wire impactors, a settling plate, and a sample coupon or additional settling plate. The flowtube was instrumented with platinum-platinum rhodium thermocouples. Hydrogen partial pressure was also monitored.

\section{Test Fuel}

In all of the tests, the fuel pins were composed of a 1 m-high stack of $\mathrm{UO}_{2}$ pellets, clad in Zircaloy-4, and pre-irradiated in the Belgian BR-3. Peak burnups were about $35 \mathrm{GWd} / \mathrm{T}$ in the pins used in STEP$1,-3$, and -4 and about $31 \mathrm{GWd} / \mathrm{T}$ in the pins used in STEP-2. During the preirradiation, the fuel power level had been relatively low for the fuel in STEP-1, intermediate for the fuel in STEP-2, and high in the fuel for STEP-3 and STEP-4. The fuel had cooled for several years prior to being tested in TREAT. These test fuel elements simulated the central portion of much-longer PWR or BWR elements.

\section{Conditions}

STEP-1 simulated the conditions of a large-break loss-of-cooling-accident (LOCA) with assumed failure of the emergency core cooling system (ECCS), the "AD" sequence; it was thus performed at low system pressure. STEP- 2 simulated BWR conditions due to failure of both the high-pressure ECCS and the long-term decay heat removal system, the "TQUW" sequence; it was also performed at low system pressure. STEP-3 and STEP-4 both simulated conditions resulting from transients in PWR due to failure of feedwater systems combined with failure to recover electric power, the station blackout "TMLB" sequence; both were performed at high system pressure (about $8 \mathrm{MPa}$ ). A simulated PWR silver-indium-cadmium control rod was included in STEP-4. The incoming steam in all tests was at $644 \mathrm{~K}\left(700^{\circ} \mathrm{F}\right)$ and at a flow rate predicted for the accident being simulated. TREAT power was provided over approximately 20 minutes at a level that would cause test-fuel fission power needed to simulate decay heating and to offset heat losses from the fuel-pin bundle. A novel TREAT reactor control scheme was developed for these tests, involving both automatic control and manual control of the transient and control rods. 


\section{Results}

Calculated maximum fuel temperatures were about $2900 \mathrm{~K}$ in STEP-1, $2700 \mathrm{~K}$ in STEP-2, $2200 \mathrm{~K}$ in STEP-3, and (with the power reduction caused by the simulated control-rod material) considerably lower than $2200 \mathrm{~K}$ in STEP-4. In STEP-3 and -4, the temperatures were much lower than expected and indicated that heat had been removed upward much more than in STEP-1 and -2. Whereas pin damage in STEP-1 and -2 was severe throughout the central portion and top portion of the fuel column, in STEP-3 and -4 it was mostly limited to the upper third of the fuel column. Heat from the hydrogen generation corresponded to cladding oxidation of (approximately) 90\% in STEP-1, 70\% in STEP-2, 40\% in STEP-3, and 30\% in STEP-4. Fission products that were collected on the sample tree were cesium, iodine, tellurium, molybdenum, and rubidium. Fuel element materials tin, zirconium, and uranium were also collected. Greater amounts of fission products were released in the lowpressure tests (STEP-1 and -2) than in the high-pressure tests (STEP-3 and -4) because strong natural-convective cooling under the higher pressure resulted in a lower fuel temperature. In STEP1 and -2 , released material increased the flow-path resistance in the main exit steam line, which caused increases in system pressure. Cesium, rubidium, and a trace of iodine were the only fission products found in the STEP-3 deposits, and none were found in STEP-4 deposits. The tests provided detailed information regarding aerosol concentration and size distribution for conditions accurately simulating the early phase of severe LWR accidents. The chemistry of the deposits was complex. Information was obtained on the collocation, chemical form, and morphology of cesium and iodine and the other detected fission products. It was observed that a marked tendency exists for component elements of ceramic and stainless steel structures to be volatilized and transported in high-pressure steam but much less in low-pressure steam.

\section{Applications}

The test results enhanced the database for testing and improving source-term models in accident analysis codes: they identified volatile fission products and extended the body of nuclear aerosol characterizations, they provided data regarding the magnitudes and release rates of fission products from degraded fuel elements as well as the physical and chemical characteristics of the released fission products, and they provided information on aerosol formation and transport that can be applied to transport mechanisms of the volatiles and condensed aerosols. The results were compared with computations of release masses, compositions, and fuel temperatures at time of release.

\section{Calibrations in TREAT}

Numerous low-power and transient irradiations of flux monitor wires, and low-power irradiations of four calibration fuel pins were performed in TREAT prior to the STEP tests in order to determine the power coupling between the TREAT core and the test fuel during the planned test transients.

\section{R-series Experiments}

The R-series tests demonstrated the coolant, cladding, and fuel dynamics during unprotected loss-offlow and transient overpower conditions with unirradiated, FFTF-type oxide fuel pins in sodium flow $[46,47,48,49,50,51,52,53,54,55][56,57,58,59,60,61,62,63,64,65][66,67,68,69]$. A onepage summary of each R-series test is included in Appendix L.

\section{Number/Time}

Eight R-series experiments (R3 through R8, R9, and R12) were performed during the time period 1973 to 1977. 


\section{Purpose}

The tests were performed to provide data to validate SAS code predictions of the timing and sequence of coolant boiling and voiding, cladding melting and runoff, and fuel melting and relocation during simulated FFTF (FTR) fast-reactor unprotected loss-of flow (ULOF) and $50 \mathrm{\$} / \mathrm{s}$ unprotected transient overpower (UTOP) conditions using beginning-of-life (actually zero burnup) full-length fuel pins in a flowing-sodium environment.

\section{Approach}

The test series utilized a new TREAT experiment system (a "once-through" sodium loop) in which the sodium flow rate could be programmed to follow a prescribed coastdown timed with the TREAT power transient. Because the Mark-I and -II package-type sodium loops were not long enough to accommodate the full-length test fuel pins, and because the use of fresh (unirradiated) fuel would not require double containment for safety purposes, a fundamentally-different test-vehicle design was used, one that involved an ex-core sodium supply and receiver system connected to a simple in-pile piping loop. Because the vehicle design was new, preliminary calibration and check-out testing was performed (referred to as "tests" R1 and R2). Instrumentation was provided in the test vehicle to detect the onset of sodium boiling, the coolant voiding dynamics, fuel pin failure, fuel-coolant interaction pressure, and fuel motions. The test series was planned to sequentially build upon the results of previous tests in the series so as to provide increasing prototypicality from test to test. Thus, test R3 was a single-pin prooftest of the experiment system; the subsequent tests would use sevenpin bundles. Test R4 would show the complete ULOF meltdown sequence, whereas test R5 would show only up to the cladding relocation phase. Test R6 repeated test R4 to gain fuel motion details that were not obtained in R4, in order to support the planning of R7. Test R7 included a power burst timed to occur when fuel motion began, to represent the neutronic effect of the fuel motion. In test R8, three of the pins were pressurized to represent fission-gas-release effects after cladding failure. Tests R9 and R12 (there were no R10 and R11 tests) investigated the effects of a $50 \mathrm{\$} / \mathrm{s}$ UTOP, with R9 driving the fuel well beyond cladding failure and R12 terminating the test promptly upon cladding failure.

\section{Limitations}

The use of fresh fuel, although not representative of fuel during its in-reactor life, provided the advantage of not involving contributions to the complexity of severe-accident progression due to fuel burnup. The reduced simplicity would be advantageous to validating the accident analysis modeling that was in its early stages of development. Utilization of a small bundle of fuel pins resulted in the usual loss of prototypicality involved in TREAT experiments, compared to behaviors in full-size subassemblies of pins. Also, as typical of TREAT testing, relocation of test fuel during a transient changes the power coupling between the test fuel and the TREAT core, making it difficult to estimate the power per unit mass in the test fuel after fuel motion begins, as the fuel density affects the self shielding. In the R-series, the flowtube in each test was a thin-walled stainless steel tube, which was readily penetratable by hot fuel. When such penetration occurred, fuel-pin material became free to enter the additional volume outside the flowtube. In addition, melting of the flowtube added steel to the mass of molten cladding in the fuel-steel system being tested. Furthermore, when the flowtube breached, the non-condensible gas outside the flowtube wall generally entered under pressure and affected the on-going molten-material dynamics. Also, in all but one of the ULOF tests, no power effect from neutronic feedback from early coolant and cladding motions was included.

\section{Experiment Vehicle}

The test vehicle was the R-series loop designed specifically for these tests. The in-core part of the system was a long, pipe loop, with one leg sized to accommodate a seven pin bundle of FFTF-type fuel pins. Enough space was provided radially around the fuel pins and flow channel to accommodate 
thermocouples and a molybdenum heat barrier to protect the stainless steel primary containment pipe wall. Flow meters and pressure transducers (and, in the later tests, acoustic sensors) were also provided along the in-core test section. The ex-core part of the system consisted of sodium supply and receiver tanks and the associated equipment for heating, flow control, etc. The flowtube in each test was stainless steel with $0.5 \mathrm{~mm}$ wall thickness, of circular cross section for the single-pin test R3 and hexagonal for the other tests. Radially surrounding the flowtube was a thick molybdenum tube. In test R3, the Mo tube was $9.4 \mathrm{~mm}$ radially from the flowtube; in the other tests it was only about two to three $\mathrm{mm}$ from the flowtube. In every test, the intermediate annular volume between the flowtube and Mo tube was filled with non-condensable gas.

\section{Test Fuel}

All of the tests used unirradiated FFTF-type fuel pins, having $91.4 \mathrm{~cm}$ of unirradiated $\mathrm{UO}_{2}$ fuel, in type 316 stainless steel cladding. The $\mathrm{UO}_{2}$ in the single fuel pin in $\mathrm{R} 3$ and in the six peripheral fuel pins in the seven-pin bundles of the other seven tests was $14 \%$ enriched; the $\mathrm{UO}_{2}$ in the central pins in all of the seven-pin bundles was $20 \%$ enriched to compensate for the lower power couplings in those central pins. Two unenriched $\mathrm{UO}_{2}$ pellets (together $1.3 \mathrm{~cm}$ high) were located at each end of the fuel column, separating the fuel from $14.4 \mathrm{~cm}$-long Inconel reflector rods at each end. Above the upper reflector rod was a spring-loaded plenum tube within the cladding, and then the 2.5 -cm top end cap. Below the lower reflector rod was the $3.5-\mathrm{cm}$ lower end cap. The overall length of the fuel pin (element) was $237 \mathrm{~cm}$. In test R8 (only), three of the seven pins were pressurized to represent fission gas pressure.

\section{Conditions}

The initial thermal-hydraulic conditions in each test were selected such that, within the first three or four seconds after reaching nominal power, temperatures in the fuel and coolant would approximately represent normal operating conditions in a high-power FFTF subassembly. For the ULOF tests, this was following by a programmed reduction in sodium flow rate, but (except for test R7) with no change in TREAT power until scram. Test R7 included a power excursion to represent the reactivity effect of fuel slumping. For the UTOP tests, on the other hand, the initial preheat was followed by a power transient simulating an FFTF $50 \mathrm{q} / \mathrm{s}$ reactivity insertion, and no change in applied inlet sodium pressure was made. The timing of test termination by reactor scram depended upon the objectives of each individual test.

\section{Results}

The results of the tests are described in terms of the timing of the events characteristic of the sequences (boiling onset, flow reversal, cladding failure, cladding and fuel motions, inlet and outlet flow blockage formation, pressure pulses) and final condition and distribution of materials. In the ULOF tests R3 through R7, cladding failures occurred between 0.9 and $1.2 \mathrm{~s}$ following inlet flow reversal, and flowtube failure occurred 2.0 to 2.5 seconds after cladding failure. In ULOF test R8 (with three of the pins pressurized), cladding failure occurred $0.7 \mathrm{~s}$ after flow reversal, and the flowtube failed $0.08 \mathrm{~s}$ thereafter. In all of these ULOF tests, $30 \%$ to $100 \%$ of the flowtube in the test melted and joined the rest of the molten material. Complete inlet flow blockages occurred in all of the ULOF tests, and outlet flow blockages occurred also in tests R4, R5, and R7. The inlet flow blockages were largely once-molten steel, varying in tests R4 through R8 from 8 to $20 \mathrm{~cm}$ height (thickness). In R3, R4, and R5 there remained some segments of pellet stacks. In R7 (with the power spike) most of the fuel, and all of the adjacent cladding and flowtube, had melted. The overpower transient in UTOP test R9 continued approximately one second after inlet flow reversal occurred, causing more than $90 \%$ of the fuel to melt and $53 \%$ of the fuel to move upward beyond the original fuel region (as high as $47 \mathrm{~cm}$ beyond), and resulted in a 27-cm-high accumulation of material blocking the outlet and also a blockage at the inlet. In contrast, test R12 was programmed to automatically terminate during inlet 
flow reversal, resulting in minor failures of all seven pins, localized near the top of the active fuel region, and $90 \%$ of the fuel remaining within the cladding.

\section{Applications}

The tests were planned and their results were evaluated using SAS code computations. This is a frequent approach when the complex interacting phenomena that will occur in the TREAT experiments are not well known, understood, or predictable. The applicability of the test results in validating the computational models was limited to the extent that the models were able to describe certain aspects of the experiment that the models were never intended to address. Such aspects are particularly the effect of flowtube breach and melting, introduction of additional void space and pressurized non-condensable gas into the test fuel region when the flowtube failed, and unknown changes in fuel power density as the fuel moved. Nevertheless, the tests provided experimental evidence of important features involved in the two major accident categories simulated, and behaviors occurring early in the disruptive phase of the tests were significantly relevant to the models.

\section{Calibrations in TREAT}

Test R1 provided radiochemical data on sample power and power distributions.

Sample power was obtained from radiochemical measurement of the barium-lanthanum activity in sample pellets. Axial power shape was obtained from foils placed along the axis of the test train. Foils on the surface of the pin were used to obtain the radial maximum-to-average power ratio. Test R2 was a heat-balance run made to confirm average calibration data and to check out the flow-decay characteristics of the system. Additional calibrations (M8CAL) obtained further information important to both the ULOF tests and the UTOP tests M9 and M12 regarding axial and azimuthal power shapes at the test fuel, and included neutronic effects of a recent change in the TREAT core slotted elements.

\section{E-series Experiments}

The E-series tests investigated fuel dispersal and fuel thermal energy to coolant work conversion of fresh and preirradiated oxide fuel pins failing into flowing sodium in $\$ 3 / \mathrm{s}$ FTR overpower transients $[70,71,72,73,74,75,76,77,78,79][80,81,82,83,84,85,86]$. A one-page summary of each E-series test is included in Appendix M.

\section{Number/Time}

Seven tests (E1 through E4, E6 through E8) were performed during 1969-1974.

\section{Purpose}

The first tests in the series were intended to investigate the potential for energetic oxide fuel-coolant interaction (causing significant fuel-energy to work conversion ratio) during an unprotected LMFBR transient overpower excursion in a flowing-sodium environment beginning with intact fuel pin(s). After those tests demonstrated that energy conversion was small, more-prototypic testing was performed to simulate LMFBR $\$ 3 / \mathrm{s}$ transient overpower hypothetical accident conditions relevant to the FTR/FFTF.

\section{Approach}

The first two tests (E1 and E2) were intended to indicate whether fuel-energy to work conversion would be significant when oxide fuel pins failed in a flowing-sodium environment. Both tests used natural-burst TREAT power transients to heat single, small, unirradiated, $\mathrm{UO}_{2}$ fuel elements. In both tests, the fuel pin was surrounded by six hollow dummy pins. E1 was intended not to drive the fuel 
pin much (if any) beyond failure. Because the cladding did not fail but instead allowed extensive, inpin axial fuel motion, a modification to the pin design and additional transient energy were used for test E2, resulting in nearly-complete fuel release from the cladding and highly fragmented fuel debris, but very low energy conversion. Test E3 subjected a bundle of three, high-gas-content preirradiated $\mathrm{UO}_{2}$ fuel pins to a natural burst transient more energetic than in E1 and E2, to include the potential for fission gas to fragment fuel before the fuel was expelled from the cladding. The test resulted in considerably-more fuel-pin damage and higher energy conversion than in E2, but the conversion efficiency was still low $(\sim 0.3 \%)$. Test E4 was intended to be similar to test $\mathrm{H} 2$ by subjecting a single, fresh, mixed-oxide pin to an overpower transient, but E2 used a much-faster power rise than the rise in H2. Test E6, involving a seven-pin bundle of mixed-oxide pins (one preirradiated pin surrounded by six fresh pins), was essentially identical to test $\mathrm{H} 4$ in that both tests used a "shaped" transient that included a short pre-heat segment followed by an overpower burst, but the burst in E6 simulated a hypothetical \$3/s reactivity accident in FFTF/FTR whereas the burst in H4 simulated a $50 / \mathrm{s}$ accident. Test E7 basically repeated E6 but with all seven of the pins in the bundle having been preirradiated, characterized by a high-power microstructure. Test E8 was nearly identical to E7 but tested a seven-pin bundle of low-power-microstructure preirradiated pins.

\section{Limitations}

The sodium loops used in the tests were not specifically designed to facilitate posttest computations of the work done by postfailure coolant dynamics caused by fuel-to-coolant heat transfer. This, combined with some questionable flowmeter data, led to significant uncertainties in the hydraulic analysis, but since the energy conversion was found to be consistently low, the level of uncertainty did not affect the general conclusion that energetic fuel-coolant interactions would not be a significant safety issue for the range of conditions studied in the tests. The test series was generally exploratory. Prototypicality (representation of subassembly conditions) regarding post-failure fuel motion was limited but was improved in the later tests E6-E8 by including additional coolant flow channels (initially using dummy "pins," progressing to a preirradiated pin surrounded by six fresh fueled pins, then to seven fueled pins). Nevertheless, because only short fuel pins were available, and because the loop design could accommodate only short pins, the hydraulic prototypicality in the tests (radially and axially) remained limited. For example, in test E6 the flow area per pin was 30\% larger than in an FTR assembly, and the axial pressure gradient was about $10 \%$ of that in an FTR assembly, deficiencies which significantly affected the prototypicality of post-failure coolant motions. In addition, the ability of hot fuel to readily melt the steel flowtube caused non-prototypic addition of molten steel (adding to melted cladding and spacer wires) to the system. Melt-through of the flowtube opened pathways for through which fuel and molten steel could move laterally into surrounding void spaces, and allowed contact between the molten materials and the surrounding, relatively-cool, steel outer wall. Such effects influenced postfailure material motions, freezing, and blockage formation. Use of a combination of both fresh and preirradiated fuel pins within the same bundle caused diminished the prototypicality of the postfailure material motions (although final distribution of the pre-irradiated fuel was determined by radiological measurement).

\section{Experiment Vehicle}

All seven tests were performed in Mark-II integral sodium loops designed for TREAT meltdown experiments on up to seven-pin bundles of EBR-II-length fuel pins. Test trains (containing the experiment fuel pin(s), the flow tube surrounding the pins, and associated structure) were inserted into the main vertical pipe of the loop. Each test train was designed specific to the number and type of fuel pins to be used in the test: one in E4, three in E3, and seven in the other tests. The loops (above and below the region occupied by the test fuel) a flowmeter, pressure transducer, and thermocouples. Additional thermocouples were included in the test trains. The loops were positioned at the center the TREAT core such that the midheight of the test fuel was located at the midheight of the core. 


\section{Test Fuel}

Tests E1, E2, and E3 used unirradiated $\mathrm{UO}_{2}$ fuel. The fuel column height was $305 \mathrm{~mm}$ in E1 and E2 and $145 \mathrm{~mm}$ in E3. Tests E4, E6, E7, and E8 tested U-Pu oxide fuel (MOX) fuel. Only one, unirradiated, pin was tested in E4. In E6 a single preirradiated pin was surrounded by six fresh pins. Seven-pin bundles or preirradiated pins were tested in E7 (high-power fuel microstructures) and E8 (lowpower fuel microstructure). The fuel column heights were $305 \mathrm{~mm}$ in E1 and E2, $145 \mathrm{~mm}$ in E3, and $343 \mathrm{~mm}$ in E4, E6, E7, and E8. In all of the tests the cladding was stainless steel (OD $=7.37 \mathrm{~mm}$ in E1 and $\mathrm{E} 2,4.42 \mathrm{~mm}$ in $\mathrm{E} 3$, and $5.84 \mathrm{~m}$ in $\mathrm{E} 4, \mathrm{E} 6, \mathrm{E} 7$, and E8).

\section{Conditions}

All of the tests started at $\sim 400+/-20^{\circ} \mathrm{C}$. The initial sodium flow velocity from test to test ranged from 3.3 to $5.4 \mathrm{~m} / \mathrm{s}$, with corresponding flow rate per pin ranging from $80-160 \mathrm{~cm}^{3} / \mathrm{s}$. The power transients in all of the tests included a sharp power burst with full-width-at-half-maximum of $0.15 \mathrm{~s}$ (initial period of $35 \mathrm{~ms}$ ) in E3 and ranging from $0.23 \mathrm{~s}$ to $0.32 \mathrm{~s}$ in the other tests (initial periods ranging from $66 \mathrm{~ms}$ to $189 \mathrm{~ms}$ ). The transients in tests E1 and E2 were simple, reactivity-step transients (the only type possible at that time, since the computer-controlled reactivity control system had not yet been installed in TREAT). The transients in the later tests used a short, initial constant-power phase to preheat the test fuel prior to the power burst, with burst full-width-at-halfmaximum in the range $230-310 \mathrm{~ms}$

\section{Results}

In test E1, the fuel pin did not fail even though up to 80 areal\% of the fuel had melted and the cladding strain reached $1.1 \%$ along the fuel column region. In-pin axial fuel motion was extensive, with some fuel reaching the top of the pin plenum. Results from the other tests in the series consistently showed (based upon coolant voiding dynamics) that the thermal-to-work energy conversion due to fuelcoolant heat transfer upon oxide fuel failing into flowing sodium was acceptably low, for fresh fuel and for irradiated fuel with low-power-structure and with high-power structure (including relativelylow and high fission-gas retention in the fuel). Conditions causing cladding failure were demonstrated, and in some tests, axial location of failure was also demonstrated. Axially unrestrained fuel could be greatly dispersed axially prior to (and preventing) cladding failure. Fuel pins that were more of the FFTF design, on the other hand, failed prior to much in-pin fuel motion and resulted in released fuel and molten cladding forming substantial blockages of the coolant channel at the top and/or bottom of the fuel column. Intimate mixing of fuel and steel was generally not observed. Penetration of the flowtube caused unprototypical additional complexity in the subsequent fuel and cladding motions, including eventual formation of blockages.

\section{Applications}

The pre-failure and early-post-failure behavior of fresh and irradiated LMFBR oxide fuel under fast transient heating aided the evaluation of computational models and accident analysis code(s), for which little or not previous in-pile test results in a flowing sodium system were available.

\section{Calibrations in TREAT}

Determinations of the neutronic coupling between the test fuel and the TREAT core during the experiments were made by irradiations of fresh pins at relatively low power and energy in TREAT and by computations.) The fuel irradiations were followed by measurements of fissions density in the fuel samples. In some cases, small foils were placed around the fresh fuel pin(s) and analyzed postirradiation to determine the azimuthal variation of neutron flux around the pin. In the later test(s), monitor wires were irradiated both at low-power steady-state (as were the fresh pins) and separately during the power transients planned for the tests, to determine the how much the neutronic coupling 
changes during the transient. The power coupling for test $\mathrm{E} 4$ was determined using measurements from the calibration measurements made for TREAT test H-1. Tests E6 and E7 used results from the H-3 power calibration measurements. The calibration for E8 involved the full range of monitor-wire and fresh fuel irradiations and measurements; it also involved using a newly-developed ultrasonic technique for cutting annular rings from several pellets from a fresh pins irradiated in TREAT calibration runs, followed by radiological analysis of the relative fissions per ring, yielding a measure of the radial fission profile in the test fuel.

\section{$16 \mathrm{H}$-series Experiments}

The H-series tests investigated the response of MOX fuel of various pre-irradiation conditions when subjected to FFTF $50 \mathrm{\$} / \mathrm{s}$ to $1 \$$ /s unprotected overpower transient simulations in flowing sodium $[83,56,80,87,88,70,89,90,91,92][93,94,95,96,97]$. A one-page summary of each H-series test is included in Appendix $\mathrm{N}$.

\section{Number/Time}

Six H-series experiments were performed during the time period 1970 to 1977.

\section{Purpose}

The test series addressed fuel response (cladding failure, initial fuel and cladding relocations, and coolant energetics) to conditions representative of hypothetical $50 \mathrm{c} / \mathrm{s}$ to $1 \$ / \mathrm{s}$ unprotected FTR overpower transients. The tests were intended to extend the earlier, preliminary/scoping overpower tests of fast-reactor mixed-oxide fuel to include fuel types and transient conditions specific to the FFTF Fast Test Reactor. This required greater prototypicality regarding the fuel design and fuel preirradiation conditions, and also regarding the test fuel power-time history and spatial power distribution and coolant environment during the transient test.

\section{Approach}

Fuel pins based on the FFTF fuel design, but short enough to be preirradiated in EBR-II and accommodated in the available Mark-II TREAT sodium loop, would be tested using fuel pins spanning a range of pre-irradiation conditions including fresh, low-to-intermediate power irradiation, and high-power irradiation. Variation of the transient behavior due to the associated range of fuel microstructural and fission-gas retention characteristics would be investigated, such as conditions causing cladding failure and ensuing initial fuel and cladding relocations. Improved prototypicality relative to earlier overpower transient tests would be provided by using a combination of features such as fast-flux irradiated fuel, multi-pin bundles, thermal-neutron-filtered test vehicles to provide better radial and axial fission-density profiles in the test fuel, shaped TREAT power transients, and ability to scram the TREAT reactor upon indications of cladding failure during the transient. Some of these features had only recently become available.

\section{Limitations}

The Mark-II integral TREAT sodium loop design had been optimized for testing small bundles of available pre-irradiated fuel pins under fairly-prototypic conditions causing cladding failure within the capability of the TREAT reactor. The loop-reactor system was limited practically to test seven-pin bundles of highly-enriched fuel pins if the pins were to be heated to failure. The limited inventory of suitable pre-irradiated fuel pins resulted in the need, in some of the tests, to test the pre-irradiated pin with six surrounding fresh pins to help simulate a prototypic radial thermal-hydraulic environment. Test conditions were computationally designed to be as prototypic as possible using available thermal-hydraulics and transient fuel behavior models which, as with the existing empirical basis, were still in their early stages of development, resulting in considerable uncertainty in 
predictive capability. In addition, experimental techniques for performing tests of this type were based on a limited foundation of prior testing experience upon which to design such experiments. Test fuel and cladding temperature distributions were less prototypic in the two tests (H1 and H2) that did not include a preheat phase before the power burst. On the other hand, the use of thermal neutron filtering and neutron-attenuating axial shaping collars on the loops helped to make the radial and axial power profiles in the test pins more prototypic. The use of fueled pins (although unirradiated) surrounding the pre-irradiated fuel pin in some respects tended to provide a moreprototypic environment for that pin, if the pre-irradiated pin should fail prior to the fresh pins; however, the capability to clearly detect which pin type (pre-irradiated or fresh) in the bundle failed first was not generally present. Extended post-failure material motions in such mixed-bundle tests, although providing useful evidence of multi-pin bundle behavior, would not fully display the behavior representative of a bundle of all identically-pre-irradiated pins. Thin stainless steel structures (such as the flowtube wall around the pins) contacted by hot fuel during a test could readily be melted through, thereby adding to the inventory of molten steel in the system and allowing molten materials to penetrate into regions outside the flowtube, creating non-prototypic material relocation scenarios.

\section{Experiment Vehicle}

Mark-II integral flowing-sodium loops were used in all of the H-series tests. Thermal-neutronabsorbing layers (flux "shaping collars") were located around the loop periphery to provide a representative axial power profile in the test fuel. Test train (fuel holder) designs varied from test to test, depending on whether one pin ( $\mathrm{H} 1$ and $\mathrm{H} 2$ ) or seven pins ( $\mathrm{H} 3$ through $\mathrm{H} 6)$ were tested and also depending upon design assumptions made regarding potential for post-failure heat transfer and material motions. The flowtube (approximately $0.8 \mathrm{~mm}$ thick) and surrounding outer tube in each test train was made of stainless steel. The test train designs for the three tests in which post-failure in-channel fuel motions were significant $(\mathrm{H} 2, \mathrm{H} 4$, and $\mathrm{H} 6)$ had the following features (moving radially outward from the flowtube): in $\mathrm{H} 2$ the flowtube was surrounded by a $0.55-\mathrm{mm}$ wide evacuated annulus, then a $0.8-\mathrm{mm}$-thick stainless steel tube, then a $10-\mathrm{mm}$ thick sodium annulus; in $\mathrm{H} 4$ the flowtube was surrounded by 2.4 -mm-wide helium gap, then a 0.9 -mm-thick stainless steel tube; in H6 the flowtube was surrounded by an inert-gas annulus and then a stainless steel tube. In the sevenpin tests, the flowtube periphery was fluted in six azimuthal places to partially represent the geometry of an additional ring of pins and thus provide a more-prototypical local flow area around the periphery of the bundle. The vehicles were outfitted with flowmeters, pressure transducers, and thermocouples.

\section{Test Fuel}

Except for test H1, all of the tests used mixed-oxide fuel pins that were like standard FFTF-fuel pins but shorter (38 cm fuel column height in $\mathrm{H} 1,34 \mathrm{~cm}$ in the other tests). In $\mathrm{H} 1$, the fuel pin was of PFR design and was tested in TREAT oriented with the plenum down, toward the coolant inlet. All pins had type 316 stainless steel cladding. In $\mathrm{H} 1$ and $\mathrm{H} 2$, the single pins tested were fresh. In each of the tests $\mathrm{H} 3, \mathrm{H} 4$, and $\mathrm{H} 5$, a single pre-irradiated fuel pin was tested as the central pin in a seven pin bundle with the other six (peripheral) pins being fresh. In H6, a bundle of seven pre-irradiated pins was tested. The pre-irradiated pins had the following irradiation characteristics: in H3, H4, and H5 the fuel had been irradiated to 3.4 to 4.4 at.\% burnup at about $35 \mathrm{~kW} / \mathrm{m}$ linear power, whereas in $\mathrm{H} 6$ the fuel had been irradiated to 6 at.\% burnup at $30 \mathrm{~kW} / \mathrm{m}$. The fuel in the $\mathrm{H} 3$ and $\mathrm{H} 5$ pre-irradiated pins had intermediate-power microstructure, whereas the pre-irradiated fuel in $\mathrm{H} 4$ had high-power microstructure, and in $\mathrm{H} 6$ the fuel had low-to-moderate-power microstructure.

\section{Conditions}

All of the tests were performed using flowing sodium under constant sodium-pumping conditions and subjected the test fuel to overpower transients. In $\mathrm{H} 1$ and $\mathrm{H} 2$, the transients were natural burst 
transients generated by an initial reactivity step in TREAT. The other four tests, using improved, computer control of the reactor, included an initial, short lower-power segment to preheat the test fuel prior to the burst. The burst shapes were similar among all the tests but can nevertheless be roughly compared according to their full-width-at-half-maximum durations: $0.36 \mathrm{~s}$ in $\mathrm{H} 1,1.3 \mathrm{~s}$ in $\mathrm{H} 2$, $\sim 0.6 \mathrm{~s}$ in $\mathrm{H} 3,1.06 \mathrm{~s}$ in $\mathrm{H} 4,1.0 \mathrm{~s}$ in $\mathrm{H} 5$, and $\sim 1.5 \mathrm{~s}$ in H6. The transients differed in part because the test-fuel power levels developed during TREAT transients typically depend not only upon the TREAT power but also upon the power coupling between the test fuel and the TREAT core specific to the particular test fuel and loop hardware design used in the test, which differed from test to test. For each test, the test transient was designed to produce fuel conditions closely approaching, or else definitely exceeding, the fuel pin failure threshold, depending upon the test objective. Significant fuel energy generation after initial cladding failure was intended to be avoided in order to preserve evidence of the early post-failure state and distribution of the fuel and cladding.

\section{Results}

The tests displayed a wide range of outcomes, including pins that did not fail (H1 and H3), pins that experienced only mild failure (H5), and pins that were grossly disrupted (H1, H4, and H6). Because of the shaped transient used in tests $\mathrm{H} 3$ through $\mathrm{H} 6$, the pre-irradiated test-fuel linear power to failure among those tests can be reasonably compared: $140 \mathrm{~kW} / \mathrm{m}$ without failure of the $\mathrm{H} 3$ intermediate-power-structure pin, $170 \mathrm{~kW} / \mathrm{m}$ at failure of the $\mathrm{H} 4$ high-power-structure pin, $\sim 180$ $\mathrm{kW} / \mathrm{m}$ at failure of the $\mathrm{H} 5$ intermediate-power-structure pin, and $\sim 200 \mathrm{~kW} / \mathrm{m}$ at failure of the lowto-moderate-power-structure pin. The fuel pin in $\mathrm{H} 1$ (as in H3) remained intact.

In $\mathrm{H} 3$, the pre-irradiated pin was heated just short of reaching the fuel solidus, whereas the fresh pins experienced up to $40 \%$ areal melting. Posttest the preirradiated pin provided detailed information regarding the post-transient characteristics of the fuel microstructure and retained fission-gas distributions. The intact pins from $\mathrm{H} 3$ also provided radiochemical data from which a direct determination of the power coupling between the test fuel and TREAT core during the test was made.

In $\mathrm{H} 5$, the pre-irradiated pin fortuitously experienced only a minor, localized cladding breach (at fuel height $\mathrm{x} / \mathrm{L}=0.84$ ) with minimal fuel release; the fresh pins were only slightly disrupted, and although little fuel motion occurred overall, the posttest flow rate was only $40 \%$ of the initial flow rate.

Gross fuel pin disruption occurred in $\mathrm{H} 2, \mathrm{H} 4$, and $\mathrm{H} 6$, resulting in complete (or nearly complete) flow blockages. Upward, in-pin fuel movement and mild sodium boiling occurred before cladding failure in $\mathrm{H} 2$ but not in any of the other five tests. In the tests involving large fuel motion, those large motions did not start until 0.2-0.5 s after cladding failure, although smaller motions may have occurred earlier in $\mathrm{H} 2$ in order to cause the detected flow blockage $50 \mathrm{~ms}$ after cladding failure. Large-mass movement of fuel within coolant channels to regions above or below the original fuel zone occurred only in $\mathrm{H} 2$. The flowtubes in $\mathrm{H} 2, \mathrm{H} 4$, and $\mathrm{H} 6$ breached, and much fuel moved into the surrounding space in $\mathrm{H} 4$ and H6 (but not in H2). Most of the fuel column was disrupted in $\mathrm{H} 2$ and H4, but mainly only the top half was disrupted in H6. In none of the tests involving significant post-failure fuel motion was significant coolant energy generated, and the peak pressure measured in the test series was on the order of $12 \mathrm{MPa}$ (in H6).

\section{Applications}

The tests provided some of the first in-pile results of the behavior of pre-irradiated FFTF-like fuel pins during simulations of hypothetical FFTF $50 \mathrm{q} / \mathrm{s}$ to 1 \$/s unprotected overpower transients in flowing sodium, including cladding failure threshold, transient-induced microstructural and fissiongas retention characteristics, and initial post-failure fuel and coolant dynamics. The tests also demonstrated subsequent fuel and coolant dynamics as coolant attempted to re-enter the hot fuel 
region, fuel melting of nearby steel structures, and propensity of the molten fuel and steel to form blockages in the coolant channel. The results were useful for comparing with analytical models, for helping to provide preliminary, empirical evidence of the associated transient behaviors, and to guide the design and planning of subsequent tests that would provide conditions of greater prototypicality.

\section{Calibrations in TREAT}

Measurements of the test-fuel to TREAT-core power coupling were made using various methods including irradiation of fuel pins and uranium monitor wires during low-power irradiations in TREAT, irradiation of monitor wires during high-power transients in TREAT, posttest radiochemical analysis of the H3 unfailed fuel pins, and (for H6) low-power irradiations of monitor foils placed around the periphery of fresh pins in a seven pin bundle, plus radiological measurements of radial segments from those pins.

\section{EOS-series Experiments}

The EOS-series tests probed the potential for fast reactor MOX fuel to disperse by fission gas pressure prior to generating high fuel vapor pressure during extremely fast high-power transients [98, 99, 100, $101,102,103]$. A one-page summary of each EOS-series test is included in Appendix 0.

\section{Number/Time}

Three EOS-series tests were performed during 1977.

\section{Purpose}

The EOS tests were performed to provide scoping information regarding whether, during rapid energetic power bursts in fast reactors, fission gas in irradiated mixed-oxide fuel can significantly disperse fuel prior to the generation of high fuel vapor pressure. The data were needed to support modeling of fuel motion in the target range of fuel heating rates, within which little was known at that time. In particular, for such power bursts, answers to the following question were sought in the tests, for both moderate-power irradiated fuel and high-power irradiated fuel: (a) does fission gas provide a dispersive mechanism for the fuel or does the gas separate from the fuel without causing significant fuel dispersal?, (b) if the fission gas does cause significant dispersal, what is the timing of that dispersal relative to the fuel reaching its solidus (or what fuel specific energy is needed to initiate dispersal or slumping in irradiated fuel versus in fresh fuel), and what mode of dispersal results?, and (c) if fission gas does not cause significant dispersal, does the fuel slump before significant fuel vapor pressure develops? The mode of fuel dispersal would depend largely on whether the fission gas had first caused grain boundary separation as the fuel solidus was being approached, resulting in formation and dispersal of finely-divided solid particles (dust) or whether the fission gas release occurred as the fuel liquidus was reached, causing fuel expansion and dispersal of larger masses of solid or melting fuel. If, prior to dispersal, the fuel was in the form of fine particles, its dispersal was anticipated appear as a generally uniform redistribution of fuel radially within the test capsule. Otherwise, the dispersal (of partially-melted chunks) was anticipated to be relatively localized.

\section{Approach}

The fuel dispersal characteristics of fuels having significantly-different fission gas content and distributions would be tested for comparison: fresh fuel in EOS-1, high-power-structure irradiated fuel in EOS-2, and medium-power-structure irradiated fuel in EOS-3. The fresh fuel pins would not undergo fuel melting or cladding failure, thereby allowing posttest characterization of transientinduced fuel grain growth, cracking, densification, and plasticity for fuel mechanics code calibration. The tests would be performed without coolant, simulating conditions in a voided coolant channel. 
The TREAT power transient would be fast enough to produce essentially adiabatic heating of the sample.

\section{Limitations}

Because the power transients used in the tests were so narrow and reached such high power, the planned test conditions were practically at the limit of TREAT experiment capability regarding accuracy and precision of measuring fuel motion (by the hodoscope), determining semi-empirically the test-fuel power, enthalpy, and temperature during the transient (by neutronics measurements, thermal-hydraulic computations, and physical properties information), and detecting the time of cladding failure (by the thermocouple response). The signal strength reaching the hodoscope was too low to allow the precise determination of the pin-failure time in EOS-1; in EOS-2 the useful data provided meager definitive information; in EOS-3 the signal was sufficient to permit good discernment of fuel axial displacement but not radial displacement.

\section{Experiment Vehicle}

The test vehicle used in all three tests consisted mainly of a pair of dry, instrumented capsules, each accommodating a short fuel pin within a boron nitride enclosure, bounded by sealed stainless steel containment walls. The capsules were located in tandem, located one above the other within the TREAT core. The upper capsule was intended to house the test fuel pin; the lower capsule was to house an unirradiated "control" pin.

\section{Test Fuel}

Both of the pre-irradiated mixed U-Pu oxide (MOX) pins used in EOS-2 and EOS-3 had been irradiated in EBR-II to $\sim 0.8$ at\% burnup. The pin designated for EOS-3 was a larger-diameter pin than the one designated for EOS-2. The EOS-2 pin linear power was $\sim 37 \mathrm{~kW} / \mathrm{m}$ resulting in the development of high-power-structure fuel (central void and concentric zones of columnar, equiaxed, and unrestructured grains), whereas the EOS-3 pin linear power was $\sim 27 \mathrm{~kW} / \mathrm{m}$ resulting in mediumpower-structure fuel (only zones of equiaxed and unrestructured grains). After the pins had been pre-irradiated, they were cut open, the cladding tubes were shortened, and the remaining cladding was refilled with a 10.2-cm stack of the pre-irradiated MOX pellets, with boron nitride pellets at each end of the stack. After the cladding was re-sealed (at 2 atm pressure), the cladding wall was reduced full length to only $0.13 \mathrm{~mm}$ thickness. (The fission-gas content of the fuel in the EOS-3 pin was calculated to be about 50\% greater than the fission-gas content of the fuel in the EOS-2 pin. However, considering the fission-gas density variation by fuel microstructure, it was estimated that the fissiongas-induced effects between the two pins in the EOS tests would be approximately as if the EOS-3 pin contained only $25 \%$ more gas than the EOS- 2 pin.) The fresh pin used as the test pin in EOS-1 was initially of the same design as the EOS-2 pin and was similarly modified. The fresh control pin in each test was similar to its companion test pin in the test, except that its cladding thickness had not been reduced.

\section{Conditions}

The power transient used in each test was essentially the same: a "natural" reactivity-step-input resulting in a burst rising on a 23-25 ms period and having a full-width-at-half-maximum of 110-120 ms. The power generated in the test fuel pins depended, as usual, on the magnitude and variation of the coupling between the test (or fresh/control) fuel and the TREAT core during the transient. In the EOS tests, peak test-fuel power levels reached approximately 100 times nominal power for those fuels. There was no coolant in the test. Near the power peak (when the test fuel pins failed), the time duration between reaching fuel solidus and fuel liquidus temperatures was about $20 \mathrm{~ms}$. 


\section{Results}

Disruption of the test pins began near peak power, at about the time the fuel solidus was reached in the preirradiated test fuel or the liquidus was reached in the fresh test fuel. (It was assumed that some fuel vapor pressure was needed to disperse the fresh fuel.) Test instrumentation was unable to accurately discern the onset of fuel disruption on the unusually-short time scale of events in the test. It was estimated that disruption of the medium-power-structure fuel in EOS-3 began at a fuel enthalpy roughly $100 \mathrm{~J} / \mathrm{g}$ less than the enthalpy in the high-power-structure fuel when the latter began to disrupt. It was also estimated that the pre-irradiated fuel disruption began at roughly 200$300 \mathrm{~J} / \mathrm{g}$ lower than when the fresh fuel disruption began. In EOS-3, significant axial motion of the test fuel did not occur until about $60 \mathrm{~ms}$ after the onset of axial fuel relocation (at peak power) was detected, and even larger axial fuel motion occurred during the next 10-100 ms as the power transient was decreasing below its half-power level. In all of the tests, axial test-fuel motion was generally more upward than downward. In EOS-2, upward fuel motion appeared to be more energetic or extensive than in EOS-1, the posttest appearance of the fuel material was like that in EOS-1, and posttest evidence indicated that after fuel movement ended and the fuel solidified, molten steel tended to drain downward. The remains of the fuel in EOS-3 were more porous (density about 8.4 $\mathrm{g} / \mathrm{cm}^{3}$ ) than the remains in EOS-1 and EOS-2 (9.3 to $\left.10.7 \mathrm{~g} / \mathrm{cm}^{3}\right)$. Pellets from the fresh fuel pin remained in good condition in the test, with no evidence of exterior cracks and no increase in diameter.

\section{Applications}

The test data (coupled with calculated test-fuel temperatures) appeared to provide evidence for the onset of fuel motion near the fuel solidus for preirradiated fuel and the fuel liquidus for fresh fuel during these extreme transients. The rate at which fuel mass dispersed axially relative to the rate at which the fuel temperature increased to and beyond the fuel liquidus was apparently not able to be well determined, for a variety of reasons. The role of fuel vapor relative to the roles of fission gas and steel vapor pressure in the larger irradiated-fuel dispersal events following initial cladding failure was therefore unclear.

\section{Calibrations in TREAT}

The relationship between the TREAT core power and the fission power generated in the EOS-series test and control pins was determined by irradiating fresh fuel pins (which were neutronically similar to the EOS pins to be transient tested) and enriched-uranium monitor wires in neutronic mockup hardware representing the actual test hardware. Radiological measurements of the irradiated samples, together with the measured reactor energy values, provided the information deeded to determine the associated power coupling factors as well as the axial power profile in the test fuel. Corrections to those factors due to fuel burnup for the pre-irradiated fuel were computed. In addition, analysis of annular samples cored from the enriched-uranium-bearing calibration fuel pins provided information on the radial power profile in the fuel.

\section{F-series Experiments}

The F-series tests investigated the motion of irradiated LMFBR mixed-oxide fuel following sodium voiding and dryout during a hypothetical loss-of-flow accident $[104,105,106,107,108,109]$. A onepage summary of each F-series test is included in Appendix P.

\section{Number/Time}

Five F-series tests were performed: F1 and F2 in 1974, F3 and F4 in 1980, and F6 in 1984. 


\section{Purpose}

The goal of the tests was to determine the timing and mode of fuel-column disruption of mixed-oxide fast reactor fuel during simulations of lead-subassembly conditions in hypothetical loss-of-flow accidents in LMFBRs with moderately-positive sodium void reactivity. The conditions involve fuel breakup in channels voided of sodium coolant after cladding has drained or dispersed. Potential disruption modes of the fuel column include column buckling, fuel swelling (before or after the solidus is reached), fragmentation, and liquid fuel jet formation, with strong dependence on the role of the fission gas initially retained in the fuel.

\section{Approach}

All tests were performed on single, modified, EBR-II-irradiated fuel pins located in capsules without coolant. Generally, the fuel was heated at a steady power until the fuel disrupted, except that in F2 a power burst was included at the end a steady-power preheat phase. Two different approaches were taken in the test designs in accordance with changes in test objectives. In F1 and F2, the fuel pins, after having their plena depressurized, were tested inside a surrounding, nuclear-heated wall intended to provide a more-prototypic environment for the fuel pin as it disrupted during the test. In tests F3, F4, and F6, details of the nature of initial fuel-column breakup dependence on fuel heating rate were sought by including an optical fuel-motion diagnostics system and using smaller fuel samples.

\section{Limitations}

The nuclear heated wall in F1 and F2, although having the advantage of providing a prototypicallyhot environment up to the initial fuel dispersal in F1 and F2, strongly diminished the hodoscope signal signal to background/noise ratio. Melting of the $\mathrm{UO}_{2}$ in the nuclear heated wall in $\mathrm{F} 2$ added $\mathrm{UO}_{2}$ to the fuel $\mathrm{UO}_{2}$ mass and tended to confuse the interpretation of the fuel motion that followed. Lack of prior knowledge regarding the rapidity of initial fuel breakup under these test condition resulted in the optical fuel-diagnostics system used in F3 and F4 being found to be inadequate and the improved optical system used in F6 to providing limited useful information. The significant additional test-fuel energy that was generated resulted, in most cases, in gross melting and relocation of the fuel after initial fuel relocation.

\section{Experiment Vehicle}

Two different designs of test capsules were used, one for F1 and F2, the other for F3, F4, and (as modified) for F6. The F1/F2 capsule contained a single EBR-II-length fuel pin ( $34.3 \mathrm{~cm}$ fuel column) inside a $0.76-\mathrm{mm}$-thick wall composed of tungsten- $\mathrm{UO}_{2}$ cermet with the $\mathrm{U}$ enriched. The wall, which would be nuclear-heated during the test, was intended to prevent non-prototypical freezeout of fuel and cladding and also provide a well-defined geometry for fuel motion. Radially beyond the heated wall were stainless steel containment barriers. The annular space between the fuel pin and heated wall was sized to represent the cross sectional area of a coolant channel in a reactor. Thermocouples were positioned slightly above and slightly below the $34.5 \mathrm{~cm}$ active test fuel region to indicate the temperature of fuel or cladding that might arrive at those locations during the test. The F3/F4 capsule, in contrast, was designed for optical viewing (using ex-reactor high-speed cameras) the $5 \mathrm{~cm}$ test-fuel column of the fuel pins used in those tests. The capsule and optical system F3 and F4 relied on the self-illumination of the sample (which proved inadequate) and was subsequently modified for F6 to allow the use of laser illumination of the sample.

\section{Test Fuel}

The fuel in all five tests was mixed oxide pellets helium-bonded to stainless steel cladding, preirradiated in the fast flux of EBR-II. In F1 and F2 the fuel had been pre-irradiated at $38-40 \mathrm{~kW} / \mathrm{m}$, to a burnup of 2.35 at $\%$ for F1 and to 0.35 at $\%$ for F2, generating a high-power structure in both fuels, 
but with a larger central void and smaller unrestructured zone in the lower-burnup fuel. Short fuel pins, containing $5-\mathrm{cm}$ of fuel that had been preirradiated at $26-29 \mathrm{~kW} / \mathrm{m}$ to 9 at $\%$ burnup and having no central void, were tested in F3, F4, and F6.

\section{Conditions}

The TREAT power transient in tests F1, F3, F4, and F6 was a constant-power run, at generating test fuel linear power of $\sim 38 \mathrm{~kW} / \mathrm{m}$ in F1, $82 \mathrm{~kW} / \mathrm{m}$ in F3, $216 \mathrm{~kW} / \mathrm{m}$ in F4, and $\sim 90 \mathrm{~kW} / \mathrm{m}$ in F6 (prior to fuel disruption). In F2, however, the transient began with a constant-power segment generating 41 $\mathrm{kW} / \mathrm{m}$ in the test fuel for several seconds but ended with a $\sim 450 \mathrm{~ms}$ full-width-at-half-maximum power burst peaking at about eleven times the initial constant power level. There was no coolant in any of the tests.

\section{Results}

In F1 and F2, initially the cladding melted and ran down to the bottom of the fuel region, followed by extensive swelling of fuel at the ends of the fuel region and melting of the rest of the fuel. In F1, the molten fuel remained within the original fuel region, but it ran down from the top half of the fuel region into the bottom half. Scram occurred about a second after fuel motion began. The hodoscope did not indicate any large fuel-motion event. The heated wall remained intact and radially confined the molten materials. In F2 just before the power burst began, cladding had melted and drained to the bottom, leaving the fuel pellet stack standing. When between 30-50\% of the burst energy had been generated, two significant upward fuel motion events occurred involving fuel in the top half of the original fuel region, some moving more than half the length of the fuel column above the original top of the fuel. As the power dropped, much of the fuel that had moved up dropped down to the bottom half of the fuel pin. At an unknown time during the transient, the $\mathrm{UO}_{2}$ phase of the heated wall melted, added its mass to the fuel $\mathrm{UO}_{2}$ inventory, and allowed a considerable amount of fuel to leak out into the surrounding space. In F3 and F4, the fuel pin disintegrated before the fuel had melted, and its disintegrationed was too fast (within $\sim 1.5 \mathrm{~ms}$ ) for the details to be discerned by the optical fuel-motion diagnostics system. In F4, the disintegration may have occurred even before the cladding had melted. In F6, the fuel pin experienced a local break or tear in the still-solid cladding, followed by a sudden release of extremely hot material that filled the $2.5 \mathrm{~cm}$ viewing area within $1 \mathrm{~ms}$.

\section{Applications}

The relative timing of cladding melting, fuel melting, and initial fuel motion of irradiated fuel in dry coolant channels as illustrated by these F-series tests allowed for conclusions to be drawn regarding the role of fission gas in fuel disruption and initial fuel dispersal under the imposed test conditions. In F1 and F2, fuel motion began after the cladding had drained off. In F1, it appeared that fission gas did not prevent an axial fuel collapse nor cause a gross axial dispersion of fuel prior to the fuel collapse that occurred, but a froth of fuel and fission products likely retarded fuel collapse until the fission products separated from the partially-molten fuel. The highly-swollen fuel radially filling the space inside the heated wall at the top and bottom of the original fuel region may have prevented molten fuel from moving past those locations. In F2, much fuel was initially driven upward far above the top of the original fuel region. Tests F3, F4, and F6 showed that initial fuel disruption may occur prior to melting of the fuel or cladding and that release of a substantial amount of hot material from the cladding can occur on the scale of one or two milliseconds. Data directly showing the mode of fuel disruption was not able to be obtained.

\section{Calibrations in TREAT}

The relationship between the TREAT core power and the fission density in the test fuel sample located within the two different F-series in-core experiment hardware systems was radiochemically determined from samples of fresh fuel, and enriched-uranium monitor wires and foils, that had been 
irradiated in TREAT within a neutronic mockup of each F-series test vehicle. Concentric samples that were core-drilled from the TREAT-irradiated fresh fuel were also radiochemically analyzed for fissions. The coupling between the heated wall in F1 and F2 and the TREAT core was similarly determined.

\section{D-series Experiments}

The D-series tests demonstrated pre-failure response of fresh FFTF-like $\mathrm{UO}_{2}$ fuel pins subjected to near-steady, localized overpower causing substantial fuel melting but with cladding remaining cool [110]. A one-page summary of each D-series test is included in Appendix Q.

\section{Number/Time}

Two D-series tests (D1 and D2) were performed in 1971.

\section{Purpose}

The tests intended to empirically-determine the conditions required to achieve the release of a small amount of molten fuel from failure of a fresh $\mathrm{UO}_{2}$ fuel pin of FFTF-like design into sodium coolant under conditions approximating normal coolant flow and temperature, and to provide information useful in determining the mechanism and cause of the fuel release, the extent of any moltenfuel/coolant interaction, the nature of any flow impediment caused by the fuel release, and whether any damage to adjacent pins tends to propagate additional pin failure.

\section{Approach}

A seven-pin bundle of fresh, FFTF-like fuel pins fueled with $\mathrm{UO}_{2}$ would be tested in flowing sodium in a constant-power transient with the power level and sodium coolant flow sufficient to likely cause cladding breach by the end of the transient. A key feature of the test would be to generate over-heating of a small section of the central pin in the bundle by over-enrichment of a few pellets of fuel near the fuel-column axial midplane. When none of the pins failed in D1, the test was repeated (test D2) using an identical but untested central pin and a 70-75\% higher power in a second attempt to cause pin failure.

\section{Limitations}

These were the first seven-pin tests performed in flowing sodium in a TREAT loop and were performed without the benefit of a significant empirical basis upon which to predict conditions for fuel pin failure in flowing sodium. The phenomenological details associated with failure of fresh fuel in the range of moderate overpower thermal conditions utilized in the tests were not well known at the time.

\section{Experiment Vehicle}

Both tests used Mark-IIA integral sodium loops, with seven-pin test trains, instrumented with flow meters, pressure transducers, and thermocouples.

\section{Test Fuel}

All pins in both tests were of FFTF-like design, but with short $(34.3 \mathrm{~cm})$ fuel columns, containing fresh $\mathrm{UO}_{2}$ pellet stacks helium bonded to type 316 stainless steel cladding. So that the fuel in the central pin would have about the same fission density during the test as the fuel in the six edge pins, the $\mathrm{UO}_{2}$ in the central pin had a higher enrichment (26 at\%) than the fuel in the edge pins (20 at\%). To cause localized cladding failure in the central pin, several $\mathrm{UO}_{2}$ pellets, occupying a 3.8-cm length at the fuel midplane of the central pin, were fully-enriched to 93 at\%. 


\section{Conditions}

Both tests subjected the test fuel to near-constant power for several seconds, with the fuel pin bundle cooled by flowing sodium. The conditions in D1 were $40 \mathrm{~kW} / \mathrm{m}(82 \mathrm{~kW} / \mathrm{m}$ in the fully-enriched section) for $\sim 13 \mathrm{~s}$ with $850 \mathrm{~cm}^{3}$ sodium flow at an inlet temperature increasing from $415^{\circ} \mathrm{C}$ to $570^{\circ} \mathrm{C}$. The conditions in D2 were $\sim 70 \mathrm{~kW} / \mathrm{m}(\sim 140 \mathrm{~kW} / \mathrm{m}$ in the fully-enriched section $)$ with $870 \mathrm{~cm}^{3}$ sodium flow at an inlet temperature that increased from $425^{\circ} \mathrm{C}$ to $555^{\circ} \mathrm{C}$.

\section{Results}

The test fuel pin remained intact in each test despite considerable fuel melting. In the region of 93\% enriched fuel, the fuel melting was up to 55 areal\% in D1 and up to 75 areal\% in D2. The maximum melting in the $26 \%$-enriched region in the central pin in D2 was 40 areal\%. In some locations, molten fuel had moved through spaces in the pellets and reached the cladding where it froze, causing practically no effect on the cladding. The outlet sodium temperature at the end of the power transient in $\mathrm{D} 2$ was $690^{\circ} \mathrm{C}$.

\section{Applications}

The two tests showed that, regarding fresh MOX fuel pins of prototypical FFTF design, short-term pin failure due to local over-enrichment of fuel is not likely to occur during near-steady fuel linear powers up to $140 \mathrm{~kW} / \mathrm{m}$ if the cladding is adequately cooled. Correspondingly, the tests provided evidence that fresh FFTF-design MOX-fueled pins can operate at nearly-normal coolant conditions for short periods of time with large amounts of molten fuel and not fail. In addition, significant useful information gained from these first TREAT sodium loop tests on seven-pin bundles guided the performance of later loop tests.

\section{Calibrations in TREAT}

The ratio of test-fuel fission density to TREAT core integrated power was determined by the irradiation of a seven-pin bundle of fresh fuel pins under low-power and also the irradiation of monitor wires under low-power and power transient conditions, and then radiologically analyzing the irradiated samples to determine fissions per gram. Posttest thermal-hydraulic calculations tended to verify the experimentally-determined test-fuel-to-core power coupling ratio.

\section{S-series Experiments}

The S-series tests investigated the energetics due to thermal interaction between oxide fuel and sodium following cladding failure, over severe fuel heating scenarios and coolant conditions at cladding failure $[111,112]$. A one-page summary of each S-series test is included in Appendix R.

\section{Number/Time}

Nine S-series (S-2 through S-8, S-11, and S-12) were performed during 1968-1972.

\section{Purpose}

The tests were performed to investigate the pressure pulses and their impulses that would be generated upon release of hot uranium oxide from fuel-rod cladding upon cladding failure. They were mainly scoping tests to determine whether the power-excursion-driven meltdown of multi-pin arrays of clad oxide fuel in sodium would result in the very high pressures (perhaps up to several hundred $\mathrm{MPa}$ ) and the high efficiency of thermal-to-mechanical energy conversion (perhaps as high as 30\%) that were calculated to be thermodynamically possible in such a system. Posttest estimations would be made of the efficiency of the conversion of test-fuel thermal energy to mechanical work that occurs during such fuel-sodium interactions. Heating conditions necessary to cause cladding failure were to 
be discovered early in the investigation. Determining the dependence of the energetics upon the fuel conditions (particulate or molten) and coolant conditions (temperature and void fraction) at the time of cladding failure, plus coolant channel geometry was a key part of the investigation. The size distribution of fuel particulate in posttest debris would help in developing an understanding of the fuel-coolant heat transfer characteristics that occurred. The test results would be compared with results of ex-reactor tests involving injection of molten $\mathrm{UO}_{2}$ into sodium.

\section{Approach}

All of the tests used short, unirradiated, stainless-steel clad uranium-oxide-fueled fuel rods immersed in stagnant sodium within strong autoclave-type test capsules. The power transients used in all of the tests were short-duration bursts (in the range 104 to $220 \mathrm{~ms}$ full-width-at-half-maximum) generated by a step-input of TREAT reactivity and controlled only by the natural negative temperature feedback reactivity of the TREAT core. Coolant temperatures, pressures, and pressure impulse energies were measured. Four tests (S-2 through S-5) used nine-rod bundles comprised of five fueled rods and four dummy rods (hollow cladding tubes) in a square array. Three tests (S-6 through S-8) used bundles of seven fueled rods in a hexagonal array. Two tests (S-11 and S-12) used single fueled rods.

\section{Limitations}

Achieving fuel-rod cladding failure in stagnant sodium prior to sodium boiling required a high fuelheating rate, generally starting from a low coolant temperature. Because the capability of predicting cladding failure conditions for helium-bonded uranium oxide fuel under severe power transients was still in an early stage of development, the in-reactor testing program initially involved some degree of trial and error in gaining the required knowledge. TREAT power bursts were limited by the available reactivity, transient rod speed, and negative neutronic feedback characteristics of the reactor. Power-pulse width, varying inversely with total pulse energy, limited the range of options for achieving the desired fuel radial temperature/melting profile and coolant temperature at the time the cladding reached its local failure temperature-pressure condition.

\section{Experiment Vehicle}

The basic capsule used in all of the tests was a robust, cylindrical, stainless steel pipe that served as the primary containment wall of the stagnant-sodium test vehicle. The inside of the pipe provided a cavity of $3.8 \mathrm{~cm}$ diameter and $34 \mathrm{~cm}$ long to accommodate the fuel rod or rod bundle and Zircaloy- 2 liner that formed the radial boundary of the region that contained the fuel rods and sodium. A piston was located at the end of the cavity and was connected to a linear motion transducer. Outside the primary containment were heaters, insulation, and (for tests S- 4 through S-12) a $0.63 \mathrm{~cm}$-thick graphite annulus, all surrounded by a thin-walled stainless steel secondary containment. A pressure transducer and several thermocouples were also positioned to measure sodium conditions.

\section{Test Fuel}

All fuel rods were about $34 \mathrm{~cm}$ long and contained $\sim 15-\mathrm{cm}$-high stacks of enriched $\mathrm{UO}_{2}$ pellets in stainless steel cladding. All contained one atmosphere pressure at room temperature, except for the fuel rods in test $S-5$. In test $S-5$, the fuel rods were evacuated to prevent the possibility of release of the fill gas at cladding breach from blanketing the interaction between released fuel and coolant.

\section{Conditions}

All tests were performed with burst-type transients. The transients in tests S- 2 through S-7 had initial periods in the range 34 to $62 \mathrm{~ms}$ and full-widths-at-half-maximum in the range $175-220 \mathrm{~ms}$. The transient in test S-8 had initial period of $27 \mathrm{~ms}$ and FWHM of $140 \mathrm{~ms}$. Tests S-11 and S-12 used transients of $23 \mathrm{~ms}$ initial period and $104 \mathrm{~ms}$ FWHM. Temperatures of the sodium and fuel rods at 
the start of each test $\left(165-190^{\circ} \mathrm{C}\right.$ in tests $\mathrm{S}-2$ through $\mathrm{S}-5, \sim 500^{\circ} \mathrm{C}$ in tests $\mathrm{S}-6$ through $\mathrm{S}-8$, and $140^{\circ} \mathrm{C}$ in tests S-11 and S-12), and incorporation of heat sinks radially surrounding the sodium, were chosen to achieve the desired combination of fuel, cladding, and sodium temperatures at the predicted time of cladding breach, taking into consideration the associated rise-time of the power burst that needed to be used for the test. The initial pressure in the capsule was $0.1 \mathrm{MPa}$ in all tests. The transients in S2 through S-8 generated between approximately 1.9 and $3.3 \mathrm{~kJ} / \mathrm{g}$ of fuel, whereas the transients in S11 and S-12 generated approximately $7 \mathrm{~kJ} / \mathrm{g}$ of fuel. In several of the tests, a considerable fraction of the total energy was generated after the cladding failed: about $30 \%$ post-failure energy in S-4 and S7 , nearly $50 \%$ in S8, and about $70 \%$ in S-11 and S-12. Analyses showed that only in test S-3 was sodium boiling likely to have occurred prior to cladding failure, that the possibility of prefailure boiling was marginal in tests S-7 and S-8, and unlikely in tests S-4, S-5, and S-6. In tests S-11 and S12 , the shortest burst possible in TREAT was used so that a high fuel energy would be reached well before the cladding exterior temperature reached the sodium boiling point.

\section{Results}

In the tests, the measured pressure-time impulse correlated well with the observed change in momentum of the piston. Peak recorded pressures (less than $20 \mathrm{MPa}$ ), fuel particulate size (generally greater than 100 microns), and calculated thermal-to-mechanical energy conversions (less than $0.2 \%$ ) indicated molten-fuel-coolant interactions that were non-explosive and of low efficiencies, under the fuel-coolant contacting conditions that actually occurred. The actual conditions, particularly the sodium temperature and amount of sodium vapor formation immediately preceding cladding failure, were not well known, however. This was due to uncertainties in temperature measurements, limited utility of data from the linear motion transducers (which locked in place before the full energy of the sodium slug could be absorbed), and two-dimensional heat-generation and heat-transfer effects that were not sufficiently analyzed. The most-finely-divided fuel residues were resulted from test $\mathrm{S}-5$ in which the fuel pins had been evacuated, thereby nearly completely eliminating the role of fill gas in the fuel-coolant interaction. In test $\mathrm{S}-8$, a mobile slurry of $\mathrm{UO}_{2}$ in molten steel with very high thermal conductivity apparently was formed.

\section{Applications}

The peak fuel-sample power levels generated in the experiments were in the range of interest for FFTF hypothetical core disassembly accident studies, but they were significantly-higher than for FFTF transient overpower excursions. The fraction of solid to molten fuel at the time of cladding breach, combined with the unprototypically-high coolant temperatures at the time of cladding breach, plus the relatively-large heat-sink area, affected the direct applicability of the results. The low energy conversion ratios indicated in all of the tests were in all cases much less than theoretical maximum ratios. Tests S-11 and S-12 demonstrated that fuel fragmentation by fuel vapor pressure, followed by intimate fuel-coolant mixing and rapid heat transfer needed for an energetic fuel-coolant interaction, did not occur.

\section{Calibrations in TREAT}

A preliminary test designated S-1 was performed using a low-power transient in TREAT to determine the power coupling between the test fuel and the TREAT core. That coupling was measured by relating the fission-energy input to a test fuel sample and the fission energy generated by the core. In addition, wet-chemical analysis of the fuel from one of the pins that remained intact during transient test S-2 provided a measure of the power coupling factor. Similar calibration irradiations were performed (one prior to test S-6, another prior to test S-7, and a third prior to tests S-11 and S-12) to determine the power coupling values for those different test-vehicle designs and fuel sample configurations. 


\section{RX-series Experiments}

The RX-series tests were performed to investigate the behavior of a mixture of molten $\mathrm{UO}_{2}$ and boiling steel with internal fission heating simulating the "transition phase" of an LMFBR accident $[113,114]$. A one-page summary of each RX-series test is included in Appendix S.

\section{Number/Time}

Two RX-series tests (RX1 and RX2) were performed during 1982-1983.

\section{Purpose}

The tests were to provide scoping information regarding the boil-up of a molten UO2-steel pool under both "open pool" and "closed pool" conditions, with internal pool heating simulating the decay heat level during the "transition phase" of a hypothetical LMFBR accident. (The open versus closed condition refers to the amount of void volume above the pool.)

\section{Approach}

In the tests, a column of fuel-steel mixture containing enriched $\mathrm{UO}_{2}$ would be fission heated at a constant rate within a fission-heated $\mathrm{UO}_{2}$ boundary resulting in the fuel melting and generation of steel vapor. Axial movement of the boiling fuel-steel pool would be monitored by the TREAT fast neutron hodoscope and by a variety of thermocouples located a several axial positions within and above the initial fuel region.

\section{Limitations}

These were the first tests of their kind and involved considerable uncertainty regarding the type of pool behavior that would be observed. Temperature instrumentation would need to be located where they would be in contact with the pool, requiring the need for them to withstand the extremely-high temperatures. Performance capability of the selected ultrasonic thermometer and its boron nitride protective sheath under such uncertain conditions was not well known. Use of a nuclear heated wall was also an experimental feature for which little prior experience existed.

\section{Experiment Vehicle}

Both tests used test vehicles of nearly identical design. In each, a cylindrical stack of enriched- $\mathrm{UO}_{2}$ steel slugs (the "fuel") was surrounded by an enriched- $\mathrm{UO}_{2}$ cylinder of $24.6 \mathrm{~mm}$ inner diameter and $4.8 \mathrm{~mm}$ thick (the nuclear-heated wall). Radially outward from the nuclear wall were thin heat shields composed, successively, of tungsten, molybdenum, graphite, and stainless steel, all within stainless steel primary and secondary containments. High temperature thermocouples were located at various elevations above the fuel and in vertical holes drilled within the fuel. In RX1, an ultrasonic thermocouple, capable of measuring temperatures at several axial locations, extended into the top of the fuel within a BN sheath. Thermal-neutron filters were placed around the region where fuel would possibly be present as it disrupted during the test, to help control the fission power density in the fuel mass as it moved. The free volume above the fuel was $\sim 5$ liters in $\mathrm{RX} 1$ and $\sim 0.5$ liter in $\mathrm{RX} 2$, to provide conditions representative of "open pool" and "closed pool," respectively.

\section{Test Fuel}

The fuel consisted of a stack of ten slugs of a hot-pressed mixture of fresh $87.6 \mathrm{wt} \% \mathrm{UO}_{2}-12.4 \mathrm{wt} \%$ stainless steel. Each slug was $2.33 \mathrm{~cm}$ diameter and $1.27 \mathrm{~cm}$ high. In most of the slugs, the $\mathrm{UO}_{2}$ was enriched to $\sim 93 \%$. The $\mathrm{UO}_{2}$ in the top and bottom slugs in $\mathrm{RX} 1$, and in the bottom slug in $\mathrm{RX} 2$, were enriched to only $50 \%$, however. Vertical holes were drilled in the slugs as needed to accommodate the thermocouples (and BN sheath in RX1). 


\section{Conditions}

The fuel in each test was heated a nearly-constant heating rate of $\sim 20 \mathrm{~W} / \mathrm{g}$ up to the time of significant fuel relocation. (As test fuel reconfigures during TREAT tests, its power coupling with the TREAT core correspondingly changes.) The power transient lasted for about $150 \mathrm{~s}$. Fission heating of the nuclear heated wall during the transient helped to provide a hot environment for the fuel.

\section{Results}

The main fuel motion began in both tests at roughly 110-120 s into the transient. In RX1, at the time of initial motion, steel vaporization had been predicted to begin. Also at that time, however, the boron nitride sheath of the ultrasonic thermocouple reached a temperature where it began decomposing, thereby removing heat needed for its decomposition and adding considerable nitrogen gas to the system. (It could not be determined whether the decomposition occurred within pool or only above the pool.) The top of the fuel moved upward several times, ultimately reaching a level $\sim 22 \mathrm{~cm}$ above its initial location. Test RX2 did not include the ultrasonic thermometer or the BN sheath. In that test, the top of the fuel pool moved gradually upward by $1.6 \mathrm{~cm}$ during the first $90 \mathrm{~s}$ of the test. The main fuel-motion of the test, however, was of the upper quarter of the fuel column moving radially beyond the heated wall to the tungsten heat shield and then downward. By the end of the test, a small amount of fuel had also moved to a point $12 \mathrm{~cm}$ above the initial location of the fuel top.

\section{Applications}

In each test, an artifact significantly complicated the test and added considerable complexity, uncertainty, and loss of prototypicality. The BN decomposition in RX1 interfered with drawing conclusions regarding the role of steel vapor, since the nitrogen gas may have been partly generated within the pool and, in any case, would have increased the system pressure enough to affect the steel vaporization rate. In RX2, the penetration of the heated wall, which allowed some of the pool to leak radially to into a cooler annular region, affected the analysis of the fuel pool axial movement. Nevertheless, the tests provided new and unique evidence of pool behavior against which analyses could be compared and assumptions evaluated.

\section{Calibrations in TREAT}

Power coupling between the TREAT core and the RX test fuel and nuclear wall was measured by irradiating identical samples of the test fuel and nuclear wall in TREAT under low-power conditions. The resulting coupling factors were then adjusted by the ratio of fissions generated in monitor wires irradiated under low power and power transient conditions intended for the RX tests. Radial and axial variation of the power coupling were computed.

\section{$22 \mathrm{~J}$-series Experiments}

The J-series investigated the response of pre-irradiated FFTF-like mixed-oxide fuel pins to the slowest transient overpower simulation possible in TREAT that results in cladding failure of such pins [115, $116,117]$. A one-page summary of each J-series test is included in Appendix T.

\section{Number/Time}

Only one test (J1) was performed, in 1979.

\section{Purpose}

The first test in the series was intended to show that a slow-period LMFBR transient overpower simulation causing cladding failure in an FFTF-like MOX fuel pin could be performed within the limitations of TREAT. The results would provide evidence of the failure threshold and post-failure fuel 
motions of preirradiated MOX fuel during such a transient. The test results would also be used in planning subsequent tests on full-length MOX fuel pin bundles in a longer, Mark-III sodium loop.

\section{Approach}

The J1 test subjected a seven-pin bundle of identical pre-irradiated mixed-oxide FFTF-like MOX fuel pins to conditions representing the slowest hypothetical unprotected FFTF overpower transient that could be simulated in TREAT and would (per pretest calculations) definitely cause failure of the test fuel pins. The TREAT power transient would be automatically terminated upon initial cladding failure, assumed to be indicated by a decrease in inlet flow rate of at least $50 \%$.

\section{Limitations}

Due to pin-to-pin self shielding, the power generated in the central pin was only $81 \%$ of the power generated in the edge pins, and there was a significant azimuthal power variation within each edge pin. (There was little power difference among the edge pins, however: $+/-4 \%$.)

\section{Experiment Vehicle}

The test vehicle was a Mark-II integral flowing-sodium loop instrumented with pressure transducers and flow meters at the inlet and outlet. Dysprosium layers around the test vehicle periphery hardened the neutron spectrum and helped to provide a representative axial power profile in the test fuel. The test train within the loop included $\sim 0.8$-mm-thick stainless steel flowtube that accommodated a seven-pin bundle. The flowtube was fluted inward in six azimuthal places to partially represent the geometry of an additional ring of pins and thus provide a more-prototypical local flow area around the periphery of the bundle. Surrounding the flowtube was a stainless steel outer tube. Between the flowtube and outer tube was an inert-gas annulus in which thermocouples and strain gauges were located, attached to the outer surface of the flowtube.

\section{Test Fuel}

The seven fuel pins contained stacks of $75 \% \mathrm{UO}_{2}-25 \% \mathrm{PuO}_{2}$ (79 at $\%$ enriched $\mathrm{U}$ ) dished pellets, with total fuel height of $34.3 \mathrm{~cm}$. The pellet stacks were each clad in $5.84 \mathrm{~mm}$ diameter, $0.38 \mathrm{~mm}$ wall, $20 \%$ cold-worked 316 stainless steel tubing. All pins had been pre-irradiated in a fast (EBR-II) spectrum to $\sim 7$ at $\%$ burnup.

\section{Conditions}

The J1 power transient consisted of a $1.3 \mathrm{~s}$ preheat at about $70-75 \mathrm{~kW} / \mathrm{m}$ (peak axial) in the edge pins followed by a linear power rise for $3.5 \mathrm{~s}$ reaching $\sim 100-110 \mathrm{~kW} / \mathrm{m}$ at pin failure, whereupon the power was rapidly dropped to a low value. The sodium coolant flow rate was $900-940 \mathrm{~cm}^{3} / \mathrm{s}$ prior to flow disruption at pin failure.

\section{Results}

Three of the seven pins failed, all being edge pins of the bundle. Prior to failure, some of the fuel columns of the edge pins had elongated by up to $\sim 3 \mathrm{~cm}$. The failures were no more than $\sim 3 \mathrm{~cm}$ long and extended axially slightly into the region occupied by $\mathrm{UO}_{2}$ insulator pellet above the fuel. The failures involved about half the circumference of the cladding. Essentially all of the fuel that was molten in those pins when they failed escaped from the cladding (a total of $\sim 53$ grams from the three pins) and remained near the failure sites. After some apparently anomalous post-transient flow behavior, the final flow rate was about $70 \%$ of the initial flow rate.

\section{Applications}

The J1 test results were unique in that it was the first time in a TREAT test that oxide fuel failed and released a significant quantity of fuel and yet resulted in a coolable geometry, that is, no complete 
coolant flow blockage formed. The test also confirmed that failure of oxide fuel pins during a simulation of a slow LMFBR overpower accident could be achieved in TREAT.

\section{Calibrations in TREAT}

Because the test vehicle and fuel pin bundle in test J1 were so similar to the ones used in TREAT test $\mathrm{H6}$, the results from the $\mathrm{H} 6$ calibration were used for J1 after being adjusted for the minor difference in the as-irradiated neutronic characteristics of the different fuel pins used in the two tests. The H6 calibration included low-power irradiations of fresh pins in a seven pin bundle (including monitor foils placed around the periphery of the pins), plus low- and high-power irradiations of monitor wires. The irradiated samples were then measured for fission density, including axial and radial relative density distributions in the fuel pins. The resulting calibration factor was supported by measurements from a J1 pre-test heat balance transient using the actual test hardware.

\section{Conclusions}

TREAT was utilized for hundreds of transients tests performed on nuclear reactor fuels during its original operation from 1959 until 1994, when the reactor was placed in non-operational standby. Future operation of TREAT will benefit from the information produced during this prior utilization of the facility, which included a diverse range of experiments. Knowledge of those prior experiments provides a foundation for planning and designing new future tests.

This report provided summary information on selected sets (series) of historic experiments to illustrate the range of experiments (and TREAT's capabilities) that characterized historic TREAT. The experiment sets addressed in this report were chosen from among the several dozen experiment types or experiment series that were performed. Further information on the broader set of experiments performed in TREAT can be found in the TREXR (TREAT Experimental Relational) Database, a compendium of reference documents and data which has been developed over the past several years at ANL [6]. 


\section{References}

[1] G. A. Freund, P. Elias, D. R. Macfarlane and J. D. Geier, "Design Summary Report on the Transient Reactor Test Facility (TREAT)," ANL-6034, Argonne National Laboratory, 1960.

[2] "Reactors Designed by Argonne National Laboratory," [Online]. Available: https://www.ne.anl.gov/About/reactors/frt.shtml.

[3] DOE Office of Nuclear Energy, "Resumption of Transient Testing," 15 April 2013. [Online]. Available: https://www.energy.gov/ne/articles/resumption-transient-testing.

[4] Department of Energy, "DOE National Laboratory Resumes Operation of U.S. Transient Test Reactor," 15 November 2017. [Online]. Available: https://www.energy.gov/articles/doenational-laboratory-resumes-operation-us-transient-test-reactor.

[5] A. E. Wright, "Transient Testing of Nuclear Fuels in TREAT: An Overview," Transactions of the American Nuclear Society, vol. 112, pp. 352-354, 7-11 June 2015.

[6] "TREXR: The TREAT Experimental Relational Database," 2018. [Online]. Available: https://www.trexr.anl.gov/.

[7] A. L. Pitner, C. J. Alderman, F. E. Bard and P. K. Scanlan, Westinghouse Hanford Company, Unpublished Information, 1989.

[8] C. J. Alderman and A. L. Pitner, Westinghouse Hanford Company, Unpublished Information, 1988.

[9] A. L. Pitner, G. E. Culley and C. J. Alderman, "Axial Relocation of Oxide Fuel During Overpower Transients," in Proceedings of the Internatioanl Topical Meeting on Safety of Next Generation Power Reactors, Seattle WA, May 1-5, 1988.

[10] A. E. Wright, D. S. Dutt and L. J. Harrison, "Fast Reactor Safety Testing in TREAT in the 1980s," in Proceedings of the 1990 International Fast Reactor Safety Meeting, Snowbird, UT, August 1216, 1990 (CONF-900804-32), 1990.

[11] H. Alter, C. B. Cowking, A. E. Klickman and M. H. Wood, "Progress and Future Plans in the PFR/TREAT Safety Testing Program," in Science and Technology of Fast Reactor Safety, Vol. 1, Proceedings of an International Conference, Guernsey, UK May 12-16 (CONF-860501--15), 1986.

[12] M. H. Wood, UKAEA Winfrith, Unpublished Information, 1985.

[13] R. Herbert, UKAEA Winfrith, Unpublished Information, 1985.

[14] M. H. Wood, R. Herbert, W. J. Woods, G. E. Culley, C. A. Hinman and A. L. Pitner, "The PFR/TREAT Capsule Experiments C01, CO2, and C03," Transactions of the American Nuclear Society, vol. 47, p. 242, 1984.

[15] A. L. Davies, G. R. Bowen, K. L. Kear, J. P. Tylka and J. W. Holland, "Seven-Pin Bundle Fast TOP Tests L01 and L02," Transactions of the American Nuclear Society, vol. 47, pp. 247-248, 1984.

[16] T. H. Bauer, J. P. Tylka, A. E. Wright, A. A. Cooper, G. R. Bowen and M. H. Wood, "TUCOP Tests L04 and L06: Comparison of Irradiated and Fresh Fuel," Transactions of the American Nuclear Society, vol. 47, pp. 248-249, 1984.

[17] R. Herbert, M. H. Wood, C. W. Hunter, J. M. Kramer and A. E. Wright, "Fuel pin failure in the PFR/TREAT experiments," Science and Technology of Fast Reactor Safety, BNES, London, p. 27, 1986.

[18] T. H. Bauer, A. E. Wright, W. R. Robinson, J. W. Holland and E. A. Rhodes, "Behavior of Modern Metallic Fuel in TREAT Transient Overpower Tests," Nuclear Technology, vol. 92, pp. 325-352, 1990. 
[19] A. E. Wright, T. H. Bauer, R. K. Lo, W. R. Robinson and R. G. Palm, "Recent metal fuel safety tests in TREAT," in Int'l Conf. on the Science and Technology of Fast Reactor Safety, British Nuclear Energy Society, Guernsey, May 12-16, 1986.

[20] A. L. Pitner, P. C. Ferrell, G. E. Culley and E. T. Weber, "TS-1 and TS-2 Transient Overpower Tests on FFTF Fuel," Transactions of the American Nuclear Society, vol. 50, pp. 351-352, 1985.

[21] A. L. Pitner, Westinghouse Hanford Company, Unpublished Information, 1985.

[22] U. P. Nayak and E. J. Manthos, Westinghouse Electric Corporation, Unpublished Information, 1984.

[23] A. L. Pitner, Westinghouse Hanford Company, Unpublished Information, 1985.

[24] J. C. Carter, R. Purviance, J. F. Boland, C. E. Dickerman and J. E. Hanson, "First Loss-of-Flow Simulation on a Prototypical FFTF Fuel Pin in the TREAT Mark-IIA Loop," Transactions of the American Nuclear Society, vol. 14, p. 281, 1971.

[25] L. W. Deitrich, E. W. Barts, A. DeVolpi, C. E. Dickerman, J. G. Eberhart, J. C. Carter, A. K. Fischer, W. F. Murphy and G. S. Stanford, "Fuel Dynamics Experiments Supporting FTR Loss-of-Flow Analysis," in Proceedings of the Fast Reactor Safety Meeting (CONF-740401-P1), Beverly Hills, CA, April 2-4, 1974.

[26] A. DeVolpi, L. Deitrich, B. Feay, L. Harrison and W. Murphy, "A Loss-of-Flow Simulation with Fresh FFTF-Type Fuel Pins," Transactions of the American Nuclear Society, vol. 16, pp. 182-184, 1973.

[27] A. DeVolpi, L. Deitrich, J. Regis, G. Stanford and R. Stewart, "Comparative Fuel Motion Effects of Fresh and Preirradiated Pins in Loss-of-Flow Simulations," Transactions of the American Nuclear Society, vol. 22, pp. 430-431, 1975.

[28] A. K. Fischer, R. K. Lo and E. W. Barts, "Fuel Dynamics Loss-of-Flow Test L3 (Final Report)," Argonne National Laboratory report ANL-76-79, June, 1976.

[29] E. W. Barts, L. W. Deitrich, J. G. Eberhart, A. K. Fischer and C. C. Meek, "Summary and Evaluation - Fuel Dynamics Loss-of-Flow Experiments (Tests L2, L3, and L4)," Argonne National Laboratory report ANL-75-57, September, 1975.

[30] J. G. Eberhart, R. K. Lo and E. W. Barts, Argonne National Laboratory, Unpublished Information, 1976.

[31] E. W. Barts, L. W. Deitrich, A. DeVolpi, J. G. Eberhart, A. K. Fischer, P. H. Froehle, W. F. Murphy, D. Stahl, R. R. Stewart, J. P. Tylka and L. J. Harrison, "Loss-of-Flow Simulations with Pre-irradiated FFTF-Type Fuel Pins (Tests L3 and L4)," Transactions of the American Nuclear Society, vol. 17, p. 363, 1973.

[32] R. Simms, S. M. Gehl, R. K. Lo and A. B. Rothman, "TREAT Test L5 Simulating an LMFBR Lossof-Flow Accident with FTR-Type Irradiated Fuel," Nuclear Technology, vol. 52, pp. 228-245, 1981.

[33] R. Simms, S. M. Gehl, R. K. Lo, A. B. Rothman and R. R. Stewart, "Posttest Evaluation of Loss-ofFlow Test L5 on FFTF-type Irradiated Fuel," Transactions of the American Nuclear Society, vol. 21, p. 283, 1975.

[34] R. Simms, G. E. Marsh, A. B. Rothman and G. S. Stanford, "Loss-of-Flow Transient Reactor Test Facility Tests L6 and L7 with Irradiated Liquid-Metal Fast Breeder Reactor Type Fuel," Nuclear Technology, no. March, pp. 331-341, 1981.

[35] R. Simms, A. B. Rothman and G. S. Stanford, "Loss-of-Flow TREAT Tests L6 and L7 on Preirradiated FTR-Type Fuel," Transactions of the American Nuclear Society, vol. 33, p. 553, 1979. 
[36] R. Simms, S. M. Gehl, R. K. Lo and A. B. Rothman, "Loss-of-Flow Test LS on FFTF-Type Irradiated Fuel," Argonne National Laboratory report ANL-78-24, 1978.

[37] C. H. Bowers, A. M. Tentner and H. U. Wider, "Analysis of TREAT Tests L7 and L8 with SAS3D, LEVITATE, and PLUT02," in Proceedings of the Specialists' Workshop on Predictive Analysis of Material Dynamics in LMFBR Safety Experiments, Los Alamos, New Mexico, 1979.

[38] D. R. Porten, A. Padilla, R. E. Baars, E. H. Randkley and G. E. Culley, "PINEX-2 Experiment: Concept Verification of an Inherent Shutdown Mechanism for HCDA's," in Proceedings of the DSANS/ENS International Meeting on Fast Reactor Safety Technology, Seattle, WA, 1979.

[39] D. E. Smith, F. J. Martin and A. Padilla, Jr., "Internal Fuel Motion Phenomenology: FUMO-E Code Analysis of PINEX Experiments," Transactions of the American Nuclear Society, vol. 41, pp. 373374, 1982.

[40] F. E. Bard and J. R. Thielges, "RFT-CAL-L Mark III Loop/Prototypic Fuel Pin Calibration Experiment," in Proceedings of the American Nuclear Society Fast, Thermal \& Fusion Reactor Experiments, Salt Lake City, UT, 1982.

[41] E. T. Weber, A. L. Pitner, F. E. Bard, G. E. Culley and C. W. Hunter, "Transient Survivability of LMR Oxide Fuel Pins," in Science and Technology of Fast Reactor Safety, Vol. 1, Proceedings of an International Conference held in Guernsey 12-16 May, 1986.

[42] C. A. Hinman and J. R. Thielges, Westinghouse Handford Company, Unpublished Information, 1985.

[43] C. J. Alderman and J. R. Thielges, Westinghouse Hanford Company, Unpublished Information, 1985.

[44] C. J. Alderman and J. R. Thielges, Westinghouse Hanford Company, Unpublished Information, 1985.

[45] L. Baker, Jr., J. K. Fink, R. Simms, B. J. Schlenger and J. E. Herceg, "Source Term Experiments Project (STEP): A summary," EPRI Report, NP-5753M, 1988.

[46] R. N. Koopman, W. F. Murphy, C. L. Fink, N. A. Kramer, F. J. Testa and R. R. Stewart, "Final Report for TREAT Transient Overpower Tests R9 and R12," Argonne National Laboratory Report ANL/RAS 80-11, April, 1980.

[47] R. N. Koopman, F. J. Testa, N. A. Kramer, R. O. McNary and A. B. Rothman, "TREAT Transient Overpower Experiment R12," Transactions of the American Nuclear Society, vol. 28, pp. 482483, 1978.

[48] R. N. Koopman, B. W. Spencer, N. A. Kramer, F. J. Testa, L. A. Semenza, R. E. Holtz and A. DeVolpi, "TREAT Transient Overpower Experiment R9," Transactions of the American Nuclear Society, vol. 24, p. 269, 1976.

[49] B. W. Spencer, F. J. Testa, N. A. Kramer and O. McNary, "TREAT Loss-of-Flow Safety Test R8 with Pressurized FTR Pins," Transactions of the American Nuclear Society, vol. 24, pp. 269-271, 1976.

[50] B. W. Spencer, M. A. Grolmes, R. E. Holtz, F. J. Testa and N. A. Kramer, "Summary and Evaluation of R-Series Loss-of-Flow Safety Tests in TREAT," Proceedings of the International Meeting on Fast Reactor Safety and Related Physics, ANS/ENS, Chicago, Illinois, October 5-8, 1976, vol. IV, pp. 1647-1657.

[51] R. E. Holtz, B. W. Spencer and F. J. Testa, "TREAT Loss-of-Flow Safety Experiment R7," Transactions of the American Nuclear Society, vol. 22, pp. 424-425, 1975.

[52] R. E. Holtz, B. W. Spencer and F. J. Testa, "Summary of Fuel Motion Resulting from the R-Series Loss-of-Flow Experiments," Transactions of the American Nuclear Society, vol. 23, pp. 358-360, 1976. 
[53] C. E. Dickerman, E. W. Barts, A. DeVolpi, R. E. Holtz, W. F. Murphy and A. B. Rothman, "Recent Results from TREAT Tests on Fuel, Cladding, and Coolant Motion," Annals of Nuclear Energy, vol. 3, pp. 313-322, 1976.

[54] A. DeVolpi, E. Rhodes, R. Holtz, J. Regis and R. R. Stewart, "Fuel Movement in R3, R5, and R6 Loss-of-Flow Simulations in TREAT," Transactions of the American Nuclear Society, vol. 21, p. 288, 1975.

[55] B. W. Spencer, M. A. Grolmes, R. E. Holtz and C. E. Miller, "Cladding Motion and Blockages in RSeries Safety Experiments," Transactions of the American Nuclear Society, vol. 19, pp. 238-239, 1974.

[56] C. E. Dickerman, A. B. Rothman, A. E. Klickman, B. W. Spencer and A. DeVolpi, "Status and Summary of TREAT In-Pile Experiments on LMFBR Response to Hypothetical Core Disruptive Accidents," in Symposium on the Thermal and Hydraulic Aspects of Nuclear Reactor Safety, Vol. 2: Liquid Metal Fast Breeder Reactors, ASME Winter Annual Meeting of the American Society of Mechanical Engineers, Atlanta, GA, Nov. 27 - Dec. 2, 1977, pp. 19-50.

[57] B. W. Spencer, R. E. Holtz and F. J. Testa, "TREAT Loss-of-Flow Safety Experiment R6," Transactions of the American Nuclear Society, vol. 21, p. 287, 1975.

[58] R. E. Holtz, B. W. Spencer, F. J. Testa and N. A. Kramer, Argonne National Laboratory, Unpublished Information, 1976.

[59] W. F. Murphy and J. C. Florek, Argonne National Laboratory, Unpublished Information, 1977.

[60] G. E. Marsh, Argonne National Laboratory, Unpublished Information, 1979.

[61] W. F. Murphy and J. C. Florek, Argonne National Laboratory, Unpublished Information, 1978.

[62] C. E. Dickerman, A. B. Rothman, A. E. Klickman, B. W. Spencer and A. DeVolpi, Argonne National Laboratory, Unpublished Information, 1978.

[63] R. E. Holtz, B. W. Spencer, F. J. Testa and N. A. Kramer, Argonne National Laboratory, Unpublished Information, 1976.

[64] B. W. Spencer, Argonne National Laboratory, Unpublished Information, 1979.

[65] C. E. Dickerman, Argonne National Laboratory, Unpublished Information, 1977.

[66] R. E. Holtz, M. A. Grolmes, B. W. Spencer, C. E. Miller, N. A. Kramer, F. J. Testa and W. F. Murphy, Argonne National Laboratory, Unpublished Information, 1977.

[67] B. W. Spencer, F. J. Testa and N. A. Kramer, Argonne National Laboratory, Unpublished Information, 1975.

[68] R. E. Holtz, M. A. Grolmes, C. E. Miller, N. R. Kramer, B. W. Spencer and F. J. Testa, Argonne National Laboratory, Unpublished Information, 1973.

[69] M. A. Grolmes, R. E. Holtz, B. W. Spencer, C. E. Miller and N. A. Kramer, Argonne National Laboratory, Unpublished Information, 1974.

[70] A. B. Rothman, L. W. Deitrich, R. C. Doerner, A. E. Wright and C. E. Dickerman, "Review of TREAT Experiments in Support of Transient Overpower (TOP) Analysis for Fast Reactor Safety," in Proceedings of the Fast Reactor Safety Meeting, Beverly Hills, California (CONF-740401), April 2-4, 1974.

[71] R. C. Doerner, C. E. Dickerman, A. B. Rothman and M. E. Stephenson, "Irradiated Fuel Failure Experiment in a Fast Reactor Reactivity Accident (Test E6)," Transactions of the American Nuclear Society, vol. 17, p. 284, 1973.

[72] R. C. Doerner and A. DeVolpi, "A Failure Experiment on High-Power FFTF-Type Fuel ina Transient Overpower Accident (Test E7)," Transactions of the American Nuclear Society, vol. 18, p. 212, 1974. 
[73] G. S. Stanford, A. DeVolpi, R. Simms, H. U. Wider, L. A. Semenza, J. P. Regis and R. R. Stewart, "Fuel Motion in TREAT Transient Overpower Test E8," Transactions of the American Nuclear Society, vol. 24, pp. 272-273, 1976.

[74] R. Simms, A. B. Rothman and W. F. Murphy, "Post-Test Evaluation of Transient Overpower TREAT Test E8 on Preirradiated FFTF-Type Fuel," Transactions of the American Nuclear Society, vol. 22, pp. 425-427, 1975.

[75] R. Simms, R. K. Lo, W. F. Murphy, G. S. Stanford and A. B. Rothman, "Transient-Overpower Test E8 on FFTF-Type Low-Power-Irradiated Fuel," Argonne National Laboratory report ANL-7793, December, 1977.

[76] R. Simms, R. K. Lo, W. F. Murphy, A. B. Rothman and G. S. Stanford, "TREAT Test E8 Simulating a Liquid-Metal Fast Breeder Reactor \$3/s Overpower Accident With Irradiated Fast Test Reactor Type Fuel," Nuclear Technology, vol. 50, pp. 225-241, 1980.

[77] R. C. Doerner, W. F. Murphy, G. S. Stanford and P. H. Froehle, "Final Report of Fuel Dynamics Test E7," Argonne National Laboratory report ANL-77-25, April, 1977.

[78] R. C. Doerner, W. F. Murphy, D. Stahl and G. S. Stanford, "Failure of a High-Power Pin in a Simulated \$3/s TOP Accident," Argonne National Laboratory report ANL-78-73, August, 1978.

[79] R. C. Doerner, C. E. Dickerman and A. B. Rothman, "Irradiated Fuel Failure Experiment in a Fast Reactor Reactivity Accident (Test E6)," Transactions of the American Nuclear Society, vol. 17, p. 284, 1973.

[80] R. C. Doerner, A. B. Rothman, A. DeVolpi, C. E. Dickerman, L. W. Deitrich, D. Stahl and W. F. Murphy, "Final Summary Report of Fuel-Dynamics Tests H2 and E4," Argonne National Laboratory report ANL-76-16, February, 1976.

[81] R. C. Doerner, C. E. Dickerman, J. F. Boland, A. K. Agrawal, E. Maslowicz, R. Purviance and J. E. Hanson, "Beyond-Failure Transient Test of a Typical FFTF Fuel Element," Transactions of the American Nuclear Society, vol. 14, p. 279, 1971.

[82] L. W. Deitrich, C. E. Dickerman, F. L. Willis, R. T. Purviance, K. J. Schmidt, A. K. Agrawal, D. Stahl, A. DeVolpi, R. C. Doerner, P. H. Froehle and J. F. Boland, "Test E3 on High-Energy Transient Meltdown of Irradiated UO2 in a TREAT Mark-II Loop," Argonne National Laboratory report ANL-77-94, December, 1977.

[83] C. E. Dickerman, L. W. Deitrich, L. E. Robinson, A. B. Rothman and J. C. Carter, "Fuel Dynamics Experiments on Fast Reactor Oxide Fuel Performance Under Transient Heating Conditions Using the TREAT Reactor," Transactions of the American Nuclear Society, vol. 14 (Suppl. 1), pp. 28-29, 1971.

[84] C. E. Dickerman, L. E. Robinson, A. K. Agrawal, W. F. Murphy and A. E. Wright, "Initial TREAT Loop Experiments on LMFBR Oxide Fuel Behavior During a Power Excursion (Tests E1 and E2)," Argonne National Laboratory report ANL-8099, May, 1974.

[85] A. B. Rothman, C. E. Dickerman, R. K. Lo, R. G. Palm, L. A. Semenza, R. Simms and H. U. Wider, "Results of Recent TOP and LOF Experiments in TREAT," in Proceedings of the International Meeting on Fast Reactor Safety and Related Physics, ANS/ENS (Conf-761001), October 5-8, 1976, Vol. IV (Phenomenology), Chicago, IL, 1625-1646.

[86] C. E. Dickerman, E. W. Barts, A. DeVolpi, R. E. Holtz, W. F. Murphy and A. B. Rothman, "Recent Results from TREAT Tests on Fuel, Cladding, and Coolant Motion," Annals of Nuclear Energy, vol. 3, pp. 315-322, 1976.

[87] R. J. Page, C. L. Fink, A. B. Rothman, R. K. Lo, L. E. Robinson and P. H. Froehle, "TREAT Test H6 a 50 cent/s Transient Overpower Accident Simulation," Nuclear Technology, vol. 45, pp. 249$268,1979$. 
[88] A. B. Rothman, A. E. Wright, D. Stahl and L. J. Harrison, "First TREAT Mark-II Loop Failure Threshold Experiment with Preirradiated FFTF-Type Fuel (H-3)," Transactions of the American Nuclear Society, vol. 16, p. 181, 1973.

[89] A. B. Rothman, A. K. Agrawal, R. T. Purviance, K. J. Schmidt, J. F. Boland and R. D. Leggett, "Failure Threshold TREAT Experiment wiht an Unirradiated Prototypical FFTF Fuel Element," Transactions of the American Nuclear Society, vol. 13, no. 2, p. 652, 1970.

[90] D. Stahl, "Fission Gas Behavior in a Mild Overpower Transient," Transactions of the American Nuclear Society, vol. 17, pp. 183-184, 1973.

[91] G. S. Stanford, D. Stahl, A. E. Wright, A. DeVolpi, C. E. Dickerman, R. R. Stewart and J. P. Regis, "Fuel Motion in Transient Overpower Test H5," Transactions of the American Nuclear Society, vol. 23, pp. 356-357, 1976.

[92] H. U. Wider and A. E. Wright, "Analysis of a Sodium Reentry Event in the H4 TREAT Test," Transactions of the American Nuclear Society, vol. 22, pp. 428-429, 1975.

[93] A. E. Wright, A. B. Rothman, D. Stahl, A. K. Agrawal, L. W. Deitrich and S. S. Chen, "Final Report for the H3 Transient Overpower Failure Threshold Experiment," Reactor Analysis and Safety Division report ANL-75-32, June, 1975.

[94] A. E. Wright, L. W. Deitrich, W. F. Murphy and A. DeVolpi, "Transient-Overpower Test H4 on FFTF-Type High-Power-Irradiated Fuel," Transactions of the American Nuclear Society, vol. 19, p. 259, 1974.

[95] A. E. Wright, A. B. Rothman, C. E. Dickerman, D. Stahl, A. DeVolpi, L. J. Harrison, T. H. Hughes and A. K. Agrawal, "In-Pile Failure Threshold Experiment (H5) with Pre-Irradiated FFTF-Type Fuel," Transactions of the American Nuclear Society, vol. 18, pp. 211-212, 1974.

[96] A. E. Wright, C. L. Fink, L. R. Kelman, L. W. Deitrich, R. T. Purviance, R. W. Mouring, A. B. Rothman and C. E. Dickerman, Argonne National Laboratory, Unpublished Information, 1976.

[97] A. E. Wright, D. Stahl, G. S. Stanford, A. B. Rothman, C. E. Dickerman and L. J. Harrison, Argonne National Laboratory, Unpublished Information, 1975.

[98] R. Avery, Argonne National Laboratory, Unpublished Information, 1975.

[99] J. P. Tylka, A. B. Rothman, P. H. Froehle, G. J. Pokorny, M. E. Stephenson and A. J. Ulrich, "EOS Series of Experiments in TREAT," Transactions of the American Nuclear Society, vol. 27, p. 508, 1977.

[100] J. P. Tylka, A. B. Rothman, S. M. Gehl, R. R. Stewart, G. S. Stanford, W. F. Murphy, G. J. Pokorny, W. G. Knapp and P. H. Froehle, Argonne National Laboratory, Unpublished Information, 1980.

[101] W. F. Murphy, Argonne National Laboratory, Unpublished Information, 1978.

[102] W. F. Murphy, Argonne National Laboratory, Unpublished Information, 1979.

[103] J. P. Tylka, Argonne National Laboratory, Unpublished Information, 1977.

[104] R. G. Palm, S. M. Gehl, C. L. Fink, D. J. Dever and A. B. Rothman, "F1 Phenomenological Test on Fuel Motion," Argonne National Laboratory report ANL-78-50, May, 1978.

[105] R. G. Palm, S. M. Gehl, C. L. Fink, R. R. Stewart and A. B. Rothman, "F2 Phenomenological Test on Fuel Motion," Transactions of the American Nuclear Society, vol. 23, pp. 354-356, 1976.

[106] R. G. Palm, C. L. Fink, R. R. Stewart, S. M. Gehl and A. B. Rothman, "F2 Phenomenological Test on Fuel Motion (Interim Report)," Argonne National Laboratory report ANL/RAS 76-29, September, 1976.

[107] A. E. Klickman, D. H. Thompson, W. A. Ragland, A. E. Wright, R. G. Palm and R. J. Page, "Review of Recent ANL Safety Experiments in SLSF and TREAT," in Proceedings of the LMFBR Safety Topical Meeting (Conf-820704-51), Vol. 2, Lyon, France, July 1982. 
[108] R. G. Palm, Argonne National Laboratory, Unpublished Information, 1984.

[109] W. F. Murphy, Argonne National Laboratory, Unpublished Information, 1983.

[110] R. G. Palm, L. W. Deitrich, W. F. Murphy, G. S. Stanford and D. Stahl, Argonne National Laboratory, Unpublished Information, 1974.

[111] R. W. Wright, J. J. Barghusen, S. M. Zivi, M. Epstein, R. O. Ivins and R. W. Mouring, "Summary of Autoclave TREAT Tests on Molton-Fuel-Coolant Interactions," in Proceedings of the Fast Reactor Safety Meeting, Beverly Hills, California, April 2-4, 1974.

[112] S. M. Zivi, M. Epstein, R. W. Wright, J. J. Barghhusen, D. H. Cho, F. J. Testa, G. T. Goldfuss and R. W. Mouring, Nuclear Science and Engineering, vol. 56, pp. 229-240, 1975.

[113] R. R. Stewart, Argonne National Laboratory, Unpublished Information, 1984.

[114] R. R. Stewart, Argonne National Laboratory, Unpublished Information, 1984.

[115] R. J. Page, A. B. Rothman, G. E. Marsh, K. J. Schmidt, F. L. Hooper, C. August, E. Johanson, G. Larsen and P. H. Froehle, "Results from the J1 Low Ramp Rate TREAT Experiment," Transactions of the American Nuclear Society, vol. 34, pp. 545-546, 1980.

[116] R. J. Page, A. B. Rothman and P. H. Froehle, "Preliminary Data Report for TREAT Test J1," Argonne National Laboratory report ANL/RAS 79-27, 1979.

[117] W. F. Murphy, Argonne National Laboratory, Unpublished Information, 1983. 


\section{Appendix A: List of All Experiment Series}

Table A.1 includes brief information about each of the many test series that were performed in TREAT prior to 1994. Each series is indicated by a test prefix. The number of tests associated with a test prefix may be several, dozens, or even hundreds, but rarely only one. Where there were many tests, the range of test parameters might have been large, requiring the brief information items in the table to be stated either more generally or more extensively. For some test prefixes, not enough information about the tests was available to provide much (if any) information into the table, but that does not imply that no such information exists anywhere. 
Table A.1. Summary Information Regarding All TREAT Historic Experiment Series

\begin{tabular}{|c|c|c|c|}
\hline $\begin{array}{l}\text { Test Name } \\
\text { Prefix }\end{array}$ & Test Sample & $\begin{array}{l}\text { Imposed Environment } \\
\text { / Conditions }\end{array}$ & $\begin{array}{l}\text { General Outcome of } \\
\text { Test(s) }\end{array}$ \\
\hline A & Unclad pellet(s) & $\begin{array}{l}\text { Inert gas; overpower } \\
\text { and/or undercooling }\end{array}$ & $\left(\mathrm{TBD}^{*}\right)$ \\
\hline AIUCPH & $\begin{array}{l}\text { Fresh Na-bonded UC pins } \\
\text { in } \mathrm{Na}\end{array}$ & Overpower & $\begin{array}{l}\text { Limited fuel damage; } \\
\text { cladding failure threshold }\end{array}$ \\
\hline AN & $\begin{array}{l}\text { Thermal reactor, aluminum } \\
\text { clad, ring-shape }\end{array}$ & Flowing steam & $\begin{array}{l}\text { (only calibration was } \\
\text { performed) }\end{array}$ \\
\hline ANLPUC & Fresh gas-bonded UC pins & Inert gas; overpower & (TBD) \\
\hline ALUXHARRISON & (TBD) & (TBD) & (TBD) \\
\hline ALUXIVINS1 & (TBD) & (TBD) & (TBD) \\
\hline APED & $\begin{array}{l}\text { Fresh Na-bonded } \mathrm{UO}_{2} ; \\
\text { single pin }\end{array}$ & $\begin{array}{l}\text { Stagnant } \mathrm{Na} \text {; } \\
\text { undercooling and } \\
\text { overpower }\end{array}$ & $\begin{array}{l}\text { Limited damage; in-pin fuel } \\
\text { motion }\end{array}$ \\
\hline APT & (TBD) & (TBD) & (TBD) \\
\hline $\mathrm{AX}$ & Fresh, Na-bonded UC & Flowing $\mathrm{Na}$ & Severe damage \\
\hline BCL & Mixed nitride & (TBD) & (TBD) \\
\hline $\mathrm{BI}$ & (TBD) & (TBD) & (TBD) \\
\hline $\mathrm{C}$ & $\begin{array}{l}\text { Fast reactor } \mathrm{UO}_{2} \text {; fresh and } \\
\text { irradiated; with and } \\
\text { without cladding }\end{array}$ & Stagnant Na; overpower & $\begin{array}{l}\text { Ranged from limited fuel } \\
\text { damage to breached cladding }\end{array}$ \\
\hline CDT & $\begin{array}{l}\text { Fast reactor MOX; } \\
\text { irradiated }\end{array}$ & Flowing Na; overpower & $\begin{array}{l}\text { In-pin fuel motion; cladding } \\
\text { breach }\end{array}$ \\
\hline CEN & $\begin{array}{l}\text { Fresh (few irradiated) } \\
\text { oxides and metals in pellet, } \\
\text { rod, and plate geometries, } \\
\text { with and without cladding }\end{array}$ & $\begin{array}{l}\text { Stagnant water, steam, } \\
\text { or inert gas; } \\
\text { undercooling and/or } \\
\text { overpower }\end{array}$ & $\begin{array}{l}\text { Limited damage; cladding } \\
\text { failure threshold, fuel and } \\
\text { cladding interaction with } \\
\text { coolant }\end{array}$ \\
\hline CENT & $\begin{array}{l}\text { Fresh, unclad carbide fuel } \\
\text { with internal cooling }\end{array}$ & $\begin{array}{l}\text { Stagnant inert gas; } \\
\text { undercooling with } \\
\text { overpower }\end{array}$ & (TBD) \\
\hline $\mathrm{CO}$ & $\begin{array}{l}\text { Fast reactor MOX; fresh, } \\
\text { low, and medium burnups }\end{array}$ & $\begin{array}{l}\text { Static NaK or flowing } \\
\text { sodium; overpower; } \\
\text { undercooling plus } \\
\text { overpower }\end{array}$ & $\begin{array}{l}\text { Pre-failure fuel motion; } \\
\text { cladding failure threshold; } \\
\text { mild cladding failure; early } \\
\text { post-failure disruption }\end{array}$ \\
\hline $\mathrm{CS}$ & (TBD) & (TBD) & (TBD) \\
\hline $\mathrm{D}$ & $\begin{array}{l}\text { Fast reactor } \mathrm{UO}_{2} \text {; fresh; pin } \\
\text { bundle }\end{array}$ & Overpower & $\begin{array}{l}\text { Limited fuel damage through } \\
\text { mild cladding failure }\end{array}$ \\
\hline $\mathrm{E}$ & $\begin{array}{l}\text { Fast reactor } \mathrm{UO}_{2} \text {; fresh, } \\
\text { low, and medium burnup; } \\
\text { single pin and pin bundle }\end{array}$ & $\begin{array}{l}\text { Flowing sodium; } \\
\text { overpower }\end{array}$ & $\begin{array}{l}\text { Pre-failure fuel motion; } \\
\text { cladding failure threshold; } \\
\text { fuel and cladding disruption } \\
\text { and dispersal }\end{array}$ \\
\hline EBRMF2-2pin & (TBD) & (TBD) & (TBD) \\
\hline EBT & $\begin{array}{l}\text { Fast reactor MOX; low and } \\
\text { medium burnup; single pin } \\
\text { and pin bundle }\end{array}$ & $\begin{array}{l}\text { Static NaK or flowing } \\
\text { sodium; overpower }\end{array}$ & $\begin{array}{l}\text { Pre-failure fuel motion; mild } \\
\text { cladding failure }\end{array}$ \\
\hline EM1 & (TBD) & (TBD) & (TBD) \\
\hline EOS & $\begin{array}{l}\text { Fast reactor MOX; fresh } \\
\text { and low burnup; single pin }\end{array}$ & $\begin{array}{l}\text { Stagnant inert gas; } \\
\text { overpower }\end{array}$ & $\begin{array}{l}\text { Cladding failure and fuel } \\
\text { motions driven by fission- } \\
\text { gas or fuel vapor pressure }\end{array}$ \\
\hline
\end{tabular}




\begin{tabular}{|c|c|c|c|}
\hline $\mathrm{F}$ & $\begin{array}{l}\text { Fast reactor MOX; low and } \\
\text { medium burnup; single pin }\end{array}$ & $\begin{array}{l}\text { Stagnant inert gas; } \\
\text { overpower }\end{array}$ & $\begin{array}{l}\text { Fuel disruption and dispersal } \\
\text { driven by fuel vapor or } \\
\text { fission gas }\end{array}$ \\
\hline FA & (TBD) & (TBD) & (TBD) \\
\hline FC & (TBD) & (TBD) & (TBD) \\
\hline FCT & (TBD) & (TBD) & (TBD) \\
\hline FCX & (TBD) & (TBD) & (TBD) \\
\hline FRF & (TBD) & (TBD) & (TBD) \\
\hline GASREL & $\begin{array}{l}\text { Fast reactor MOX, medium } \\
\text { burnup }\end{array}$ & $\begin{array}{l}\text { Flowing sodium; } \\
\text { overpower }\end{array}$ & $\begin{array}{l}\text { Mild cladding failure; fission } \\
\text { gas release }\end{array}$ \\
\hline GRIST & $\begin{array}{l}\text { Gas-cooled fast reactor } \\
\text { oxide; pin bundle }\end{array}$ & Flowing helium & (test not performed) \\
\hline $\mathrm{H}$ & Fast reactor MOX & $\begin{array}{l}\text { Flowing sodium; } \\
\text { overpower }\end{array}$ & $\begin{array}{l}\text { Pre-failure fuel motion } \\
\text { through post-failure fuel and } \\
\text { cladding disruption and } \\
\text { dispersal }\end{array}$ \\
\hline $\mathrm{HC}$ & Helium-bonded carbide & $\begin{array}{l}\text { Flowing sodium; } \\
\text { overpower }\end{array}$ & \\
\hline $\mathrm{HN}$ & Helium-bonded nitride & (TBD) & (TBD) \\
\hline HOP & $\begin{array}{l}\text { Fast reactor MOX, medium } \\
\text { burnup }\end{array}$ & $\begin{array}{l}\text { Sodium (stagnant or } \\
\text { flowing); transient } \\
\text { overpower }\end{array}$ & $\begin{array}{l}\text { Mild cladding failure; fission } \\
\text { gas release }\end{array}$ \\
\hline HUCPTO & $\begin{array}{l}\text { Fast reactor MOX, medium } \\
\text { burnup }\end{array}$ & $\begin{array}{l}\text { Flowing sodium; } \\
\text { undercooling }\end{array}$ & (TBD) \\
\hline HUT & $\begin{array}{l}\text { Fast reactor MOX, low, } \\
\text { medium and high burnup }\end{array}$ & $\begin{array}{l}\text { Stagnant sodium; } \\
\text { overpower }\end{array}$ & $\begin{array}{l}\text { Pre-failure fuel damage; } \\
\text { cladding failure threshold }\end{array}$ \\
\hline IDRP & $\begin{array}{l}\text { Fast reactor U-fissium } \\
\text { alloy; fresh }\end{array}$ & Flowing sodium & (TBD) \\
\hline IRT & $\begin{array}{l}\text { Fast reactor U-fissium } \\
\text { alloy; fresh }\end{array}$ & Flowing sodium & $\begin{array}{l}\text { Instrumentation response } \\
\text { and calibration }\end{array}$ \\
\hline $\mathrm{J}$ & $\begin{array}{l}\text { Fast reactor MOX pins in } \\
\text { bundle; medium burnup }\end{array}$ & $\begin{array}{l}\text { Flowing sodium; } \\
\text { overpower }\end{array}$ & $\begin{array}{l}\text { Cladding failure and post- } \\
\text { failure material interactions } \\
\text { and motions }\end{array}$ \\
\hline $\mathrm{L}$ & $\begin{array}{l}\text { Fast reactor MOX; fresh, } \\
\text { low, and medium burnup; } \\
\text { pin bundle }\end{array}$ & $\begin{array}{l}\text { Flowing sodium; } \\
\text { undercooling and } \\
\text { undercooling+ } \\
\text { overpower }\end{array}$ & $\begin{array}{l}\text { Cladding failure, post-failure } \\
\text { material interactions and } \\
\text { motions }\end{array}$ \\
\hline LO & $\begin{array}{l}\text { Fast reactor MOX; fresh } \\
\text { and low burnup; pin bundle }\end{array}$ & $\begin{array}{l}\text { Flowing sodium; } \\
\text { overpower, } \\
\text { undercooling, and } \\
\text { undercooling+ } \\
\text { overpower }\end{array}$ & $\begin{array}{l}\text { Pre-failure fuel motions; } \\
\text { cladding failure threshold; } \\
\text { post-failure material } \\
\text { interactions and motions }\end{array}$ \\
\hline M & $\begin{array}{l}\text { Fast reactor U-fissium U-Zr, } \\
\text { and U-Pu-Zr; pin bundle }\end{array}$ & $\begin{array}{l}\text { Flowing sodium; } \\
\text { overpower }\end{array}$ & $\begin{array}{l}\text { Pre-failure fuel motions; } \\
\text { cladding failure threshold; } \\
\text { post-failure material } \\
\text { interactions and motions }\end{array}$ \\
\hline MF & (TBD) & (TBD) & (TBD) \\
\hline MFT & (TBD) & (TBD) & (TBD) \\
\hline MWT & (TBD) & (TBD) & (TBD) \\
\hline NRPA & (TBD) & (TBD) & (TBD) \\
\hline ORNL-FPR & (TBD) & (TBD) & (TBD) \\
\hline PBF & (TBD) & (TBD) & (TBD) \\
\hline PHOT062 & (TBD) & (TBD) & (TBD) \\
\hline
\end{tabular}




\begin{tabular}{|c|c|c|c|}
\hline PINEX & $\begin{array}{l}\text { Fast reactor MOX; low } \\
\text { burnup; }\end{array}$ & Static NaK & Pre-failure fuel motion \\
\hline PNL & (TBD) & (TBD) & (TBD) \\
\hline $\mathrm{R}$ & $\begin{array}{l}\text { Fast reactor } \mathrm{UO}_{2} \text {; fresh; pin } \\
\text { bundle }\end{array}$ & $\begin{array}{l}\text { Flowing sodium; } \\
\text { overpower, } \\
\text { undercooling, } \\
\text { undercooling+ } \\
\text { overpower }\end{array}$ & $\begin{array}{l}\text { Pre-failure fuel motions; } \\
\text { cladding failure threshold; } \\
\text { post-failure material } \\
\text { interactions and motions }\end{array}$ \\
\hline REMET & (TBD) & (TBD) & (TBD) \\
\hline RFT & $\begin{array}{l}\text { Fast reactor MOX; low and } \\
\text { medium burnup; single pin }\end{array}$ & $\begin{array}{l}\text { Flowing sodium; } \\
\text { overpower }\end{array}$ & $\begin{array}{l}\text { Limited fuel damage; pre- } \\
\text { failure fuel motion }\end{array}$ \\
\hline RP49 & (TBD) & (TBD) & (TBD) \\
\hline $\mathrm{RX}$ & $\begin{array}{l}\text { Fast reactor } \mathrm{UO}_{2}+\text { steel; } \\
\text { fresh fuel; monolithic mass }\end{array}$ & $\begin{array}{l}\text { No coolant; heating to } \\
\text { simulate melt-down } \\
\text { accident transition } \\
\text { phase }\end{array}$ & (TBD) \\
\hline $\mathrm{S}$ & $\begin{array}{l}\text { Fast reactor } \mathrm{UO}_{2} \text {; fresh; } \\
\text { single pin and pin bundle }\end{array}$ & $\begin{array}{l}\text { Stagnant sodium; high } \\
\text { overpower burst }\end{array}$ & $\begin{array}{l}\text { Molten fuel-coolant } \\
\text { interaction, including with } \\
\text { fuel vapor effects }\end{array}$ \\
\hline S(roman) & $\begin{array}{l}\mathrm{UO}_{2}, \mathrm{UC}, \mathrm{U} \text {-fissium and } \\
\text { other metal alloys; fresh } \\
\text { and low burnup; steel and } \\
\text { zirconium claddings; bare } \\
\text { fuel or single pin }\end{array}$ & $\begin{array}{l}\text { No coolant or stagnant } \\
\text { sodium or NaK coolant; } \\
\text { overpower }\end{array}$ & $\begin{array}{l}\text { Fuel disruption; cladding } \\
\text { failure threshold; post- } \\
\text { failure fuel dispersal }\end{array}$ \\
\hline SC & UC & $\begin{array}{l}\text { Flowing sodium; } \\
\text { overpower }\end{array}$ & $\begin{array}{l}\text { Cladding failure threshold; } \\
\text { post-failure material motions }\end{array}$ \\
\hline STEP & $\begin{array}{l}\text { LWR } \mathrm{UO}_{2} \text {; medium burnup; } \\
\text { pin bundle }\end{array}$ & $\begin{array}{l}\text { Flowing steam; } \\
\text { sustained power } \\
\text { generation after } \\
\text { cladding breach }\end{array}$ & $\begin{array}{l}\text { Fission product release, } \\
\text { chemical reactions, } \\
\text { transport, and deposition. }\end{array}$ \\
\hline Th20U* & (TBD) & (TBD) & (TBD) \\
\hline TR-1 and TR-2 & MOX & (TBD) & (TBD) \\
\hline $\begin{array}{l}\text { TR (but not TR-1 } \\
\text { or TR-2) }\end{array}$ & $\begin{array}{l}\text { U-fissium; fresh and low } \\
\text { burnup; single pin or pin } \\
\text { bundle }\end{array}$ & $\begin{array}{l}\text { Flowing sodium or } \\
\text { flowing inert gas; } \\
\text { overpower and } \\
\text { undercooling+ } \\
\text { overpower }\end{array}$ & $\begin{array}{l}\text { Cladding failure threshold; } \\
\text { post-failure material } \\
\text { interactions and motions }\end{array}$ \\
\hline TS & $\begin{array}{l}\text { Fast reactor MOX; medium } \\
\text { burnup; single pin }\end{array}$ & $\begin{array}{l}\text { Flowing sodium; } \\
\text { overpower }\end{array}$ & $\begin{array}{l}\text { Pre-failure fuel motion; } \\
\text { cladding failure threshold; } \\
\text { post-failure material } \\
\text { interactions and motions }\end{array}$ \\
\hline$\overline{U L}$ & $\begin{array}{l}\text { UC; fresh and low burnup; } \\
\text { single pin }\end{array}$ & Overpower & $\begin{array}{l}\text { Pre-failure fuel motion; } \\
\text { cladding failure threshold; } \\
\text { post-failure material } \\
\text { interactions and motions }\end{array}$ \\
\hline $\mathrm{UN} / \mathrm{PuN}$ & (TBD) & (TBD) & (TBD) \\
\hline $23 \mathrm{nT}^{1}$ & $\mathrm{LWR} \mathrm{UO}_{2}$ & $\begin{array}{l}\text { Water coolant; } \\
\text { overpower }\end{array}$ & $\begin{array}{l}\text { Cladding failure threshold; } \\
\text { post-failure material } \\
\text { interactions }\end{array}$ \\
\hline
\end{tabular}




\section{Appendix B: One-page Summaries of CDT-series Tests}

One-page summaries of each of the CDT-series tests are included here. These summaries can also be found in TREXR [6]. 


\title{
Summary of TREAT Test CDT1
}

\author{
Conducted 9 June 1987
}

Transient No. 2742

\section{PRE-TEST SAMPLE CHARACTERIZATION}

\begin{tabular}{|l|l|}
\hline \multicolumn{2}{|c|}{ FUEL } \\
\hline Form & He-bonded $\mathrm{MOX}$ \\
\hline Composition & $\mathrm{UO}_{2}$ (Natural) $-24.6 \% \mathrm{PuO}_{2}$ \\
\hline Geometry & Solid pellet \\
\hline Fissile height & $91.4 \mathrm{~cm}$ \\
\hline $\begin{array}{l}\text { Smear } \\
\text { density }\end{array}$ & $84.3 \%$ of theoretical \\
\hline
\end{tabular}

\begin{tabular}{|l|l|}
\hline \multicolumn{2}{|c|}{ CLADDING } \\
\hline Material & $\begin{array}{l}\text { Thermo-mechanical treated HT9 ferritic } \\
\text { alloy }\end{array}$ \\
\hline OD & $0.686 \mathrm{~cm}$ \\
\hline Thickness & $0.053 \mathrm{~cm}$ \\
\hline
\end{tabular}

\begin{tabular}{|l|l|}
\hline \multicolumn{2}{|l|}{ PRE-IRRADIATION CHARACTERISTICS } \\
\hline BU (peak axial) & $116 \mathrm{MWd} / \mathrm{kg}$ \\
\hline Peak linear power & $33.6 \mathrm{~kW} / \mathrm{m}$ \\
\hline $\begin{array}{l}\text { Peak internal clad } \\
\text { temperature }\end{array}$ & $575^{\circ} \mathrm{C}$ \\
\hline $\begin{array}{l}\text { Fluence } \\
\text { (spectrum) }\end{array}$ & $\begin{array}{l}1.70 \times 10^{23} \mathrm{n} / \mathrm{cm}^{2} ; \text { fast } \\
\text { spectrum }\end{array}$ \\
\hline
\end{tabular}

OTHER FUEL SAMPLE INFORMATION

CDE fuel pin w/ $\mathrm{HT} 9$ cladding from the FFTF assembly ACO-1, Plenum: $\left(23.6 \mathrm{~cm}^{3}\right.$ of $\mathrm{He}+1 \mathrm{~cm}^{3}$ of $\mathrm{Xe}+1 \mathrm{~cm}^{3}$ of $\mathrm{Kr}$ ) at $1 \mathrm{~atm}$, annular insulator pellets

\begin{tabular}{|l|l|}
\hline \multicolumn{2}{|c|}{ TEST CONDITIONS, MEASUREMENTS, \& ANALYSES } \\
\hline $\begin{array}{l}\text { Coolant } \\
\text { environment }\end{array}$ & Flowing sodium \\
\hline $\begin{array}{l}\text { Transient } \\
\text { conditions }\end{array}$ & $\begin{array}{l}\text { 10-s steady-state flattop to } 33.6 \mathrm{~kW} / \mathrm{m}, 28-\mathrm{s} 5 \mathrm{c} / \mathrm{s} \\
\text { TOP to peak power of } 160.9 \mathrm{~kW} / \mathrm{m}, \mathrm{scram}\end{array}$ \\
\hline $\begin{array}{l}\text { Fuel sample } \\
\text { configuration } \\
\text { in the test }\end{array}$ & Single pin \\
\hline $\begin{array}{l}\text { Test vehicle \& } \\
\text { experiment } \\
\text { system }\end{array}$ & Modified Mark IIIC Loop \\
\hline $\begin{array}{l}\text { Measurements } \\
\text { /diagnostics } \\
\text { during test }\end{array}$ & $\begin{array}{l}\text { Thermocouples, flowmeters, fuel motion } \\
\text { (hodoscope) }\end{array}$ \\
\hline $\begin{array}{l}\text { Posttest } \\
\text { coolant flow } \\
\text { condition }\end{array}$ & No blockage \\
\hline $\begin{array}{l}\text { Posttest } \\
\text { condition of } \\
\text { test sample(s) }\end{array}$ & $\begin{array}{l}\text { No cladding failure, severe fuel motion/voiding, } \\
\text { max. cladding strain of 2.5\% }\end{array}$ \\
\hline $\begin{array}{l}\text { Posttest } \\
\text { measurements } \\
\text { \& information }\end{array}$ & $\begin{array}{l}\text { Neutron radiography, ceramography, macro and } \\
\text { metallographic examination, gamma scanning, } \\
\text { full plenum spring compression }\end{array}$ \\
\hline $\begin{array}{l}\text { Posttest } \\
\text { analyses }\end{array}$ & $\begin{array}{l}\text { Profilometry, TEMECH and SIEX-4P code } \\
\text { comparison (pre-failure and end-of-life fuel } \\
\text { condition) }\end{array}$ \\
\hline
\end{tabular}

\section{KEY SUMMARY INFORMATION ABOUT THE TEST}

Experiment CDT-1 was conducted to investigate the effects of a LMFBR transient overpower on Core Demonstration Experiment $(\mathrm{CDE})$ type fuel pins. This test provided transient performance data which supported the irradiation of CDE in the Fast Flux Test Facility to its goal burnup of 900 equivalent full power days. In order to provide a test environment which better simulated the characteristics of a LMFBR core, the test train was designed to minimize heat transfer between the fuel/sodium region and the peripheral structure with an insulating argon-filled region, known as the adiabatic region. Unfortunately, a crack in the flowtube allowed sodium to flood into this area, causing an increase in thermal conductivity. This affected the axial and radial temperature profiles, although it was not considered an issue for test validity. In spite of the higher power level and higher measured cladding temperature of CDT-3, the solid pin design of CDT-1 displayed more transient-induced strain than the annular fuel pin of CDT-3. This is a strong indicator that the annular fuel design allows for fuel-region pressure mitigation. Approximately $5.8 \mathrm{~cm}$ of fuel motion from the fuel region toward the upper plenum region was observed, providing evidence of an inherent safety feature which could potentially lessen the consequences of an overpower transient. After posttest analysis, it was concluded that the large margin to failure and the high cladding ductility of these pins allowed them to accommodate greater strain than similar pins clad in $20 \%$ cold-worked stainless steel. It was also noted that further CDE burnup was supported by the expected reduction in the rate of transient damage associated with the increase of burnup (due to reduced power levels and therefore lower temperatures). 


\section{Summary of TREAT Test CDT2}

Conducted 11 August 1987

Transient No. 2757

\section{PRE-TEST SAMPLE CHARACTERIZATION}

\begin{tabular}{|l|l|}
\hline \multicolumn{2}{|c|}{ FUEL } \\
\hline Form & He-bonded MOX \\
\hline Composition & $\begin{array}{l}\text { ACO-1: } \mathrm{UO}_{2} \text { (natural) }-24.6 \mathrm{w}_{0} \mathrm{PuO}_{2} \\
\text { FO-2: } \mathrm{UO}_{2} \text { (depleted) }-26 \% \mathrm{PuO}_{2}\end{array}$ \\
\hline Geometry & $\begin{array}{l}\text { Pellet } \\
\text { (ACO-1 solid; FO-2 solid or annular) }\end{array}$ \\
\hline Fissile height & $91.4 \mathrm{~cm}$ \\
\hline $\begin{array}{l}\text { Smear } \\
\text { density }\end{array}$ & $\begin{array}{l}\text { ACO-1: } 84.3 \% \text { of theoretical } \\
\text { FO-2 Solid: } 84.3 \% \text { of theoretical } \\
\text { FO-2 Annular: 80\% of theoretical }\end{array}$ \\
\hline
\end{tabular}

\begin{tabular}{|l|l|}
\hline \multicolumn{2}{|c|}{ CLADDING } \\
\hline Material & $\begin{array}{l}\text { Thermo-mechanically treated HT9 } \\
\text { ferritic alloy }\end{array}$ \\
\hline OD & $0.686 \mathrm{~cm}$ \\
\hline Thickmess & $0.053 \mathrm{~cm}$ \\
\hline
\end{tabular}

\begin{tabular}{|c|c|}
\hline \multicolumn{2}{|c|}{ PRE-IRRADIATION CHARACTERISTICS } \\
\hline BU (peak axial) & $\begin{array}{l}\text { ACO-1 Solid: } 116 \mathrm{MWd} / \mathrm{kg} \\
\text { FO-2 Solid: } 64 \mathrm{MWd} / \mathrm{kg} \\
\text { FO-2 Annular: } 65 \mathrm{MWd} / \mathrm{kg}\end{array}$ \\
\hline Peak linear power & $\begin{array}{l}\text { ACO-1 Solid: } 32.9 \mathrm{~kW} / \mathrm{m} \\
\text { FO-2 Solid: } 37.3 \mathrm{~kW} / \mathrm{m} \\
\text { FO-2 Annular: } 36.0 \mathrm{~kW} / \mathrm{m}\end{array}$ \\
\hline $\begin{array}{l}\text { Peak internal clad } \\
\text { temperature }\end{array}$ & $\begin{array}{l}\text { ACO-1: } 597{ }^{\circ} \mathrm{C} \\
\text { FO-2: } 549^{\circ} \mathrm{C}\end{array}$ \\
\hline $\begin{array}{l}\text { Fluence } \\
\text { (spectrum) }\end{array}$ & $\begin{array}{l}\text { ACO-1: } 1.79 \times 10^{23} \mathrm{n} / \mathrm{cm}^{2} \\
\text { FO-2: } 0.98 \times 10^{23} \mathrm{n} / \mathrm{cm}^{2} \\
\text { Fast spectrum }\end{array}$ \\
\hline
\end{tabular}

\section{OTHER FUEL SAMPLE INFORMATION}

CDE fuel pin w/ HT9 cladding from the FFTF assemblies

$\mathrm{ACO}-1$ and FO-2, annular insulator pellets

\begin{tabular}{|c|c|}
\hline \multicolumn{2}{|c|}{ TEST CONDITIONS, MEASUREMENTS, \& ANALYSES } \\
\hline $\begin{array}{l}\text { Coolant } \\
\text { environment }\end{array}$ & Flowing sodium \\
\hline $\begin{array}{l}\text { Transient } \\
\text { conditions }\end{array}$ & $\begin{array}{l}\text { 10-s steady-state flattop to approx. } 35 \mathrm{~kW} / \mathrm{m}, 2-\mathrm{s} \\
1 \text { S/s TOP to peak power of approx. } 662 \mathrm{~kW} / \mathrm{m} \text {, } \\
\text { scram }\end{array}$ \\
\hline $\begin{array}{l}\text { Fuel sample } \\
\text { configuration } \\
\text { in the test }\end{array}$ & Three pins in separate flow tubes \\
\hline $\begin{array}{l}\text { Test vehicle \& } \\
\text { experiment } \\
\text { system }\end{array}$ & Modified Mark IIIC Loop \\
\hline $\begin{array}{l}\text { Measurements } \\
\text { /diagnostics } \\
\text { during test }\end{array}$ & $\begin{array}{l}\text { Thermocouples, flowmeters, fuel motion } \\
\text { (hodoscope) }\end{array}$ \\
\hline $\begin{array}{l}\text { Posttest } \\
\text { coolant flow } \\
\text { condition }\end{array}$ & No blockage \\
\hline $\begin{array}{l}\text { Posttest } \\
\text { condition of } \\
\text { test sample(s) }\end{array}$ & $\begin{array}{l}\text { Cladding failure and severe fuel } \\
\text { movement/voiding (FO-2 solid and annular only), } \\
\text { maximum cladding strain of } 9.5 \% \text { (ACO-1 Solid } \\
\text { only), full plenum spring compression }\end{array}$ \\
\hline $\begin{array}{l}\text { Posttest } \\
\text { measurements } \\
\& \text { information }\end{array}$ & $\begin{array}{l}\text { Neutron radiography, ceramography, macro \& } \\
\text { metallographic examination, gamma scanning }\end{array}$ \\
\hline $\begin{array}{l}\text { Posttest } \\
\text { analyses }\end{array}$ & $\begin{array}{l}\text { Profilometry, TEMECH and SIEX-4P code } \\
\text { comparison (pre-failure and end-of-life fuel } \\
\text { condition) }\end{array}$ \\
\hline
\end{tabular}

KEY SUMMARY INFORMATION ABOUT THE TEST

Experiment CDT-2 was conducted to investigate the effects of a LMFBR transient overpower on Core Demonstration Experiment (CDE) type fuel pins. This test provided transient performance data which supported the irradiation of CDE in the Fast Flux Test Facility to its goal burnup of 900 equivalent full power days. Extensive pre-failure axial fuel movement from the high-worth fuel region to the low-worth plenum region was observed in the FO-2 annular pin, providing evidence of an inherent safety feature which could potentially lessen the consequences of an overpower transient. After posttest analysis, it was concluded that the large margin to failure and the high cladding ductility of these pins allowed them to accommodate greater strain than similar pins clad in $20 \%$ cold-worked stainless steel. It was also noted that further CDE burnup was supported by the expected reduction in the rate of transient damage associated with the increase of burnup (due to reduced power levels and therefore lower temperatures). 


\title{
Summary of TREAT Test CDT3
}

\author{
Conducted 31 July 1987
}

Transient No. 2752

\begin{tabular}{l}
\hline \multicolumn{2}{|l|}{ PRE-TEST SAMPLE CHARACTERIZATION } \\
\begin{tabular}{|l|l|}
\hline \multicolumn{2}{|c|}{ FUEL } \\
\hline Form & $\mathrm{He}$-bonded MOX \\
\hline Composition & $\mathrm{UO}_{2}$ (natura) $-26.0 \%_{0} \mathrm{PuO}_{2}$ \\
\hline Geometry & Annular pellet \\
\hline Fissile height & $91.4 \mathrm{~cm}$ \\
\hline $\begin{array}{l}\text { Smear } \\
\text { density }\end{array}$ & $80.0 \%$ of theoretical \\
\hline
\end{tabular}
\end{tabular}

\begin{tabular}{|l|l|}
\hline \multicolumn{2}{|c|}{ CLADDING } \\
\hline Material & $\begin{array}{l}\text { Thermo-mechanically treated HT9 } \\
\text { ferritic alloy }\end{array}$ \\
\hline OD & $0.686 \mathrm{~cm}$ \\
\hline Thickness & $0.053 \mathrm{~cm}$ \\
\hline
\end{tabular}

\begin{tabular}{|l|l|}
\hline \multicolumn{2}{|c|}{ TEST CONDITIONS, MEASUREMENTS, \& ANALYSES } \\
\hline $\begin{array}{l}\text { Coolant } \\
\text { environment }\end{array}$ & Flowing sodium \\
\hline $\begin{array}{l}\text { Transient } \\
\text { conditions }\end{array}$ & $\begin{array}{l}10-\mathrm{s} \text { steady-state flattop to approx. 35 kW/m, 2-s } \\
\text { scram }\end{array}$ \\
\hline $\begin{array}{l}\text { Fuel sample } \\
\text { configuration } \\
\text { in the test }\end{array}$ & Three pins in separate flow tubes \\
\hline $\begin{array}{l}\text { Test vehicle \& } \\
\text { experiment } \\
\text { system }\end{array}$ & Modified Mark IIIC Loop \\
\hline $\begin{array}{l}\text { Measurements } \\
\text { /diagnostics } \\
\text { during test }\end{array}$ & $\begin{array}{l}\text { Thermocouples, flowmeters, fuel motion } \\
\text { (hodoscope) }\end{array}$ \\
\hline $\begin{array}{l}\text { Posttest } \\
\text { coolant flow } \\
\text { condition }\end{array}$ & No blockage \\
\hline $\begin{array}{l}\text { Posttest } \\
\text { condition of } \\
\text { test sample(s) }\end{array}$ & $\begin{array}{l}\text { Cadding failure and severe fuel movement/ } \\
\text { voiding (FO-2 solid and annular only), maximum } \\
\text { plenum spring compression }\end{array}$ \\
\hline $\begin{array}{l}\text { Posttest } \\
\text { measurements } \\
\text { \& information }\end{array}$ & $\begin{array}{l}\text { Neutron radiography, ceramography, macro and } \\
\text { metallographic examination, gamma scanning }\end{array}$ \\
\hline $\begin{array}{l}\text { Posttest } \\
\text { analyses }\end{array}$ & $\begin{array}{l}\text { Profilometry, TEMECH and SIEX-4P code } \\
\text { comparison (pre-failure and end-of-life fuel } \\
\text { condition) }\end{array}$ \\
\hline
\end{tabular}

\begin{tabular}{|l|l|}
\hline \multicolumn{2}{|l|}{ PRE-IRRADIATION CHARACTERISTICS } \\
\hline BU (peak axial) & $63 \mathrm{MWd} / \mathrm{kg}$ \\
\hline Peak linear power & $34.6 \mathrm{~kW} / \mathrm{m}$ \\
\hline $\begin{array}{l}\text { Peak internal clad } \\
\text { temperature }\end{array}$ & $556^{\circ} \mathrm{C}$ \\
\hline $\begin{array}{l}\text { Fluence } \\
\text { (spectrum) }\end{array}$ & $\begin{array}{l}0.94 \times 10^{23} \mathrm{n}^{-\mathrm{cm}^{2}} ; \text { fast } \\
\text { spectrum }\end{array}$ \\
\hline
\end{tabular}

OTHER FUEL SAMPLE INFORMATION

CDE fuel pins w/ HT9 cladding from the FFTF assembly

FO-2, annular insulator pellets

\section{KEY SUMMARY INFORMATION ABOUT THE TEST}

Experiment CDT-3 was conducted to investigate the effects of a LMFBR transient overpower on Core Demonstration Experiment (CDE) type fuel pins. This test provided transient performance data which supported the irradiation of CDE in the Fast Flux Test Facility to its goal burnup of 900 equivalent full power days. In order to provide a test environment which better simulated the characteristics of a LMFBR core, the test train was designed to minimize heat transfer between the fuel/sodium region and the peripheral structure with an insulating argon-filled region, known as the adiabatic region. Unfortunately, a crack in the flowtube allowed sodium to flood into this area, causing an increase in thermal conductivity. This affected the axial and radial temperature profiles, although it was not considered an issue for test validity. In spite of the higher power level and higher cladding temperature of CDT-3, the annular fuel pin of CDT-3 displayed less transient-induced strain than the solid pin design of CDT-1. This is a strong indicator that the annular fuel design allows for fuel-region pressure mitigation. The annular fuel and insulator pellets also provided a channel for approx. $10 \mathrm{~g}$ of molten fuel to escape into the upper plenum region. This pre-failure axial fuel movement from high-worth to low-worth regions associated with annular fuel pins could potentially be implemented as a passive safety measure. After posttest analysis, it was concluded that the large margin to failure and the high cladding ductility of these pins allowed them to accommodate greater strain than similar pins clad in $20 \%$ cold-worked stainless steel. It was also noted that further CDE burnup was supported by the expected reduction in the rate of transient damage associated with the increase of burnup (due to reduced power levels and therefore lower temperatures) 


\section{Appendix C: One-page Summaries of CO-series Tests}

One-page summaries of each of the CDT-series tests are included here. These summaries can also be found in TREXR [6]. 


\section{Summary of TREAT Test CO1}

Conducted 5 November 1981

Transient No. 2238

\begin{tabular}{l}
\hline PRE-TEST SAMPLE CHARACTERIZATION \\
\begin{tabular}{|l|l|}
\hline \multicolumn{2}{|c|}{ FUEL } \\
\hline Form & Helium-bonded MOX \\
\hline Composition & $\mathrm{UO}_{2}$ (natural U) - $31.5 \mathrm{wt} \% \mathrm{PuO}_{2}$ \\
\hline Geometry & Annular pellet, flat ends \\
\hline Fissile height & $91.4 \mathrm{~cm}$ \\
\hline $\begin{array}{l}\text { Smear } \\
\text { density }\end{array}$ & $80.0 \%$ of theoretical \\
\hline
\end{tabular}
\end{tabular}

\begin{tabular}{|l|l|}
\hline \multicolumn{2}{|c|}{ CLADDING } \\
\hline Material & 316 stainless steel \\
\hline OD & $0.584 \mathrm{~cm}$ \\
\hline Thickness & $0.038 \mathrm{~cm}$ \\
\hline
\end{tabular}

\begin{tabular}{|l|l|}
\hline \multicolumn{2}{|l|}{ PRE-IRRADIATION CHARACTERISTICS } \\
\hline BU (peak axial) & No pre-irradiation \\
\hline Peak linear power & (not applicable) \\
\hline $\begin{array}{l}\text { Peak internal clad } \\
\text { temperature }\end{array}$ & (not applicable) \\
\hline $\begin{array}{l}\text { Fluence } \\
\text { (spectrum) }\end{array}$ & (not applicable) \\
\hline
\end{tabular}

\section{OT HER FUEL SAMPLE INFORMATION}

Fuel: $161.7 \mathrm{~g}$; pellets natural $\mathrm{UO} 2(10 \mathrm{~cm}$ upper/solid,

$46 \mathrm{~cm}$ breeder lower/annular); bottom plenum $15.4 \mathrm{~cm}^{3}$

\begin{tabular}{|c|c|}
\hline \multicolumn{2}{|c|}{ TEST CONDITIONS, MEASUREMENTS, \& ANALYSES } \\
\hline $\begin{array}{l}\text { Coolant } \\
\text { environment }\end{array}$ & Static NaK \\
\hline $\begin{array}{l}\text { Transient } \\
\text { conditions }\end{array}$ & $\$ 5 / s$ ramp insertion \\
\hline $\begin{array}{l}\text { Fuel sample } \\
\text { configuration } \\
\text { in the test }\end{array}$ & Single full-size fuel pin \\
\hline $\begin{array}{l}\text { Test vehicle \& } \\
\text { experiment } \\
\text { system }\end{array}$ & $\begin{array}{l}\text { Single-pin capsule with a massive nickel heat } \\
\text { sink at the periphery of the NaK coolant. }\end{array}$ \\
\hline $\begin{array}{l}\text { Measurements } \\
\text { /diagnostics } \\
\text { during test }\end{array}$ & $\begin{array}{l}\text { Coolant and heat-sink temperature, fuel } \\
\text { movement (by hodoscope and PINEX camera) }\end{array}$ \\
\hline $\begin{array}{l}\text { Posttest } \\
\text { coolant flow } \\
\text { condition }\end{array}$ & $\begin{array}{l}\text { Cadding and fuel debris around pin; no flow } \\
\text { data }\end{array}$ \\
\hline $\begin{array}{l}\text { Posttest } \\
\text { condition of } \\
\text { test sample(s) }\end{array}$ & Gross disruption \\
\hline $\begin{array}{l}\text { Posttest } \\
\text { measurements } \\
\text { \& information }\end{array}$ & $\begin{array}{l}\text { Location of fuel failure, fuel and cladding } \\
\text { distribution, neutron radiography, optical } \\
\text { microscopy }\end{array}$ \\
\hline $\begin{array}{l}\text { Posttest } \\
\text { analyses }\end{array}$ & Thermal-mechanical analyses \\
\hline
\end{tabular}

\section{KEY SUMMARY INFORMATION ABOUT THE TEST}

Test CO1 was performed to determine the cladding breach timing and location during a $\$ 5 / \mathrm{s}$ reactivity insertion rate overpower transient. It was a companion test to single-pin tests $\mathrm{CO} 2$ and $\mathrm{CO} 3$, which were performed in stagnant NaK on medium-burnup and high-burnup fuel, respectively. There was no pre-failure length change of the fuel, within the hodoscope's sensitivity limits (about $+/-6 \mathrm{~mm}$ ). Post-test radiographs of the test pin revealed that severe pin disruption occurred at the bottom and axial mid-plane of the fuel column. Cladding failure occurred at roughly 24 times the nominal power of $44 \mathrm{~kW} / \mathrm{m}$ for the type of pin tested, which analysis indicated was mainly by a melt-through. There was apparently a heat transfer problem (perhaps a bubble in the NaK bond) in the capsule near the bottom of the fuel pin that enhanced a melt-through failure at that location. A related test using a seven-pin bundle of fuel pins of this type in a flowing sodium loop was test LO1. 


\section{Summary of TREAT Test CO2 \\ Conducted 23 November 1981}

Transient No. 2299

\begin{tabular}{l}
\hline PRE-TEST SAMPLE CHARACTERIZATION \\
\begin{tabular}{|l|l|}
\hline \multicolumn{2}{|c|}{ FUEL } \\
\hline Form & Helium-bonded MOX \\
\hline Composition & $\begin{array}{l}\left.\mathrm{UO}_{2} \text { (natural } \mathrm{U}\right)-31.5 \mathrm{wt} \% \mathrm{PuO}_{2} \\
\text { Oxygen-to-metal ratio }=1.985\end{array}$ \\
\hline Geometry & Annular pellet, flat ends \\
\hline Fissile height & $91.4 \mathrm{~cm}$ \\
\hline $\begin{array}{l}\text { Smear } \\
\text { density }\end{array}$ & $80.0 \%$ of theoretical \\
\hline
\end{tabular}
\end{tabular}

\begin{tabular}{|l|l|}
\hline \multicolumn{2}{|c|}{ CLADDING } \\
\hline Material & 316 stainless steel \\
\hline OD & $0.584 \mathrm{~cm}$ \\
\hline Thickness & $0.038 \mathrm{~cm}$ \\
\hline
\end{tabular}

\begin{tabular}{|l|l|}
\hline PRE-IRRADIATION CHARACTERISTICS \\
\hline BU (peak axial) & $\sim 4$ at\% of HM \\
\hline Peak linear power & $30 \mathrm{~kW} / \mathrm{m}$ equivalent \\
\hline $\begin{array}{l}\text { Peak internal clad } \\
\text { temperature }\end{array}$ & (not available) \\
\hline $\begin{array}{l}\text { Fluence } \\
\text { (spectrum) }\end{array}$ & $\begin{array}{l}\text { Fluence not available; fast } \\
\text { spectrum }\end{array}$ \\
\hline
\end{tabular}

\section{OT HER FUEL SAMPLE INFORMATION}

Fuel $159.1 \mathrm{~g}$; breeder pellets natural $\mathrm{UO}_{2}(10 \mathrm{~cm}$ upper/solid, $46 \mathrm{~cm}$ lower/annular); bottom plenum 15.4 $\mathrm{cm}^{3}$ at $4.19 \mathrm{~atm}$ (cold).

As-irradiated fuel: low-power structure, $0.9 \mathrm{~mm}$ central

hole, total fission gas release $1.61 \times 10^{-3}$ moles $=29 \%$.

\begin{tabular}{|c|c|}
\hline \multicolumn{2}{|c|}{ TEST CONDITIONS, MEASUREMENTS, \& ANALYSES } \\
\hline $\begin{array}{l}\text { Coolant } \\
\text { environment }\end{array}$ & Static NaK \\
\hline $\begin{array}{l}\text { Transient } \\
\text { conditions }\end{array}$ & $\$ 5 / \mathrm{s}$ reactivity ramp insertion \\
\hline $\begin{array}{l}\text { Fuel sample } \\
\text { configuration } \\
\text { in the test }\end{array}$ & Single full size fuel pin \\
\hline $\begin{array}{l}\text { Test vehicle \& } \\
\text { experiment } \\
\text { system }\end{array}$ & $\begin{array}{l}\text { PFR/TREAT Test Vehicle Assembly (stagnant } \\
\text { NaK capsule) }\end{array}$ \\
\hline $\begin{array}{l}\text { Measurements } \\
\text { /diagnostics } \\
\text { during test }\end{array}$ & $\begin{array}{l}\text { Coolant and heat-sink temperature, fuel } \\
\text { movements (by hodoscope and PINEX camera) }\end{array}$ \\
\hline $\begin{array}{l}\text { Posttest } \\
\text { coolant flow } \\
\text { condition }\end{array}$ & $\begin{array}{l}\text { Cadding and fuel debris around pin; no flow } \\
\text { data }\end{array}$ \\
\hline $\begin{array}{l}\text { Posttest } \\
\text { condition of } \\
\text { test sample(s) }\end{array}$ & Gross disruption \\
\hline $\begin{array}{l}\text { Posttest } \\
\text { measurements } \\
\text { \& information }\end{array}$ & $\begin{array}{l}\text { Neutron radiography, optical microscopy, } \\
\text { location of fuel failure, fuel and cladding } \\
\text { redistribution, }\end{array}$ \\
\hline $\begin{array}{l}\text { Posttest } \\
\text { analyses }\end{array}$ & Thermal-mechanical analyses \\
\hline
\end{tabular}

\section{KEY SUMMARY INFORMATION ABOUT THE TEST}

Test $\mathrm{CO} 2$ was performed to determine the cladding breach timing and location during a $\$ 5 / \mathrm{s}$ reactivity insertion rate overpower transient on low burnup ( 4 at\%, peak) fuel. It was a companion test to single-pin tests $\mathrm{CO} 1$ and $\mathrm{CO} 3$, which were performed in stagnant NaK on zero-burnup and high-burnup fuel, respectively. There was no pre-failure length change of the fuel, within the hodoscope's sensitivity limits (about $+1-6 \mathrm{~mm}$ ). Cladding failure which occurred at roughly 24 times nominal power ( $44 \mathrm{~kW} / \mathrm{m}$ for pins of this type), apparently was caused by internal pressurization and the elevated cladding temperature at the top of the pin. Some fuel cladding mechanical interaction occurred in the lower part of the pin. A related test using a seven-pin bundle of fuel pins of this type in a flowing sodium loop was test LO2. 


\section{Summary of TREAT Test $\mathrm{CO} 3$}

Conducted 2 December 1981

Transient No. 2300

\begin{tabular}{|c|c|}
\hline \multicolumn{2}{|c|}{ PRE-TEST SAMPLE CHARACTERIZATION } \\
\hline \multicolumn{2}{|r|}{ FUEL } \\
\hline Form & Helium-bonded MOX \\
\hline Composition & $\begin{array}{l}73 \text { wt\% UO2 (23.5\% enriched), } 27 \\
\text { wt } \% \mathrm{PuO}_{2}\end{array}$ \\
\hline Geometry & Annular pellet, flat ends \\
\hline Fissile height & $91.44 \mathrm{~cm}$ \\
\hline $\begin{array}{l}\text { Smear } \\
\text { density }\end{array}$ & $81.5 \%$ of theoretical \\
\hline
\end{tabular}

\begin{tabular}{|l|l|}
\hline \multicolumn{2}{|c|}{ CLADDING } \\
\hline Material & 316 stainless steel \\
\hline OD & $0.584 \mathrm{~cm}$ \\
\hline Thickness & $0.038 \mathrm{~cm}$ \\
\hline
\end{tabular}

\begin{tabular}{|l|l|}
\hline PRE-IRRADIATION CHARACTERISTICS \\
\hline BU (peak axial) & 9 at\% of HM \\
\hline Peak linear power & $\begin{array}{l}54.0 \mathrm{~kW} / \mathrm{m}(27.7 \mathrm{~kW} / \mathrm{m} \\
\text { burnup-averaged) }\end{array}$ \\
\hline $\begin{array}{l}\text { Peak internal clad } \\
\text { temperature }\end{array}$ & (not available) \\
\hline $\begin{array}{l}\text { Fluence } \\
\text { (spectrum) }\end{array}$ & $\begin{array}{l}\text { Fluence not available; fast } \\
\text { spectrum }\end{array}$ \\
\hline
\end{tabular}

\section{OTHER FUEL SAMPLE INFORMATION}

Fuel $166.6 \mathrm{~g}$; breeder pellets natural $\mathrm{UO}_{2}(10 \mathrm{~cm}$ upper/solid, $46 \mathrm{~cm}$ lower/annular); bottom plenum 15.4 $\mathrm{cm}^{3}$ at $4.19 \mathrm{~atm}$ (cold).

As-irradiated fuel: medium-to-high-power structure

\begin{tabular}{|c|c|}
\hline \multicolumn{2}{|c|}{ TEST CONDITIONS, MEASUREMENTS, \& ANALYSES } \\
\hline $\begin{array}{l}\text { Coolant } \\
\text { environment }\end{array}$ & Static NaK \\
\hline $\begin{array}{l}\text { Transient } \\
\text { conditions }\end{array}$ & $\$ 5 / s$ reactivity ramp insertion \\
\hline $\begin{array}{l}\text { Fuel sample } \\
\text { configuration } \\
\text { in the test }\end{array}$ & Single full-size fuel pin \\
\hline $\begin{array}{l}\text { Test vehicle \& } \\
\text { experiment } \\
\text { system }\end{array}$ & $\begin{array}{l}\text { PFR/TREAT Test Vehicle Assembly (static NaK } \\
\text { capsule) }\end{array}$ \\
\hline $\begin{array}{l}\text { Measurements } \\
\text { /diagnostics } \\
\text { during test }\end{array}$ & $\begin{array}{l}\text { Coolant and heat-sink temperature, fuel } \\
\text { movements (by hodoscope and PINEX camera) }\end{array}$ \\
\hline $\begin{array}{l}\text { Posttest } \\
\text { coolant flow } \\
\text { condition }\end{array}$ & $\begin{array}{l}\text { Cadding and fuel debris around pin; no flow } \\
\text { data }\end{array}$ \\
\hline $\begin{array}{l}\text { Posttest } \\
\text { condition of } \\
\text { test sample(s) }\end{array}$ & Gross disruption \\
\hline $\begin{array}{l}\text { Posttest } \\
\text { measurements } \\
\& \text { information }\end{array}$ & $\begin{array}{l}\text { Location of fuel failure, fuel movement and } \\
\text { deposition, extent of fuel-cladding mechanical } \\
\text { interaction, impact of internal pressurization, } \\
\text { neutron radiography, optical microscopy }\end{array}$ \\
\hline $\begin{array}{l}\text { Posttest } \\
\text { analyses }\end{array}$ & Thermal-mechanical analyses \\
\hline
\end{tabular}

\footnotetext{
KEY SUMMARY INFORMATION ABOUT THE TEST

Test $\mathrm{CO} 3$ was performed to determine the cladding breach timing and location during a $\$ 5 / \mathrm{s}$ ramp insertion rate overpower transient on high burnup ( 9 at\%, peak) fuel. It was a companion test to single-pin tests $\mathrm{CO} 1$ and CO2, which were performed in stagnant NaK on zero burnup and low-burnup fuel, respectively. There was no pre-failure length change of the fuel, within the hodoscope's sensitivity limits (about $+1-6 \mathrm{~mm}$ ). Cladding failure occurred at $25-45 \mathrm{~ms}$ before the TREAT power peak, at a fuel elevation about one-fourth of the fuel height (which was attributed to a problem in the experiment. Cladding failure apparently was caused by fuel cladding mechanical interaction (FCMI), but strain measurements were not made. Failure by internal pressurization was delayed relative to $\mathrm{CO} 2$ by the lower cladding temperatures. The melt-through possibility was very low for $\mathrm{CO} 3$. The low failure location (about $1 / 4$ above the bottom of the fuel column) is also consistent with an FCMI, since no unusual temperature changes were detected in that region, as they were in CO1. The cladding melted near the bottom of the fuel and completely penetrated the knitmesh plug at the fuel bottom.
} 


\title{
Summary of TREAT Test CO4
}

\author{
Conducted 15 July 1982
}

Transient No. 2370

\begin{tabular}{l}
\hline PRE-TEST SAMPLE CHARACTERIZATION \\
\begin{tabular}{|l|l|}
\hline \multicolumn{2}{|c|}{ FUEL } \\
\hline Form & Helium-bonded MOX \\
\hline Composition & $\begin{array}{l}\left.\mathrm{UO}_{2} \text { (natural } \mathrm{U}\right)-31.5 \text { wt } \% \mathrm{PuO}_{2} \\
\text { Oxygen-to-metal ratio }=1.985\end{array}$ \\
\hline Geometry & Annular pellets with flat ends \\
\hline Fissile height & $91.4 \mathrm{~cm}$ \\
\hline $\begin{array}{l}\text { Smear } \\
\text { density }\end{array}$ & $79 \%$ of theoretical \\
\hline
\end{tabular}
\end{tabular}

\begin{tabular}{|l|l|}
\hline \multicolumn{2}{|c|}{ CLADDING } \\
\hline Material & $316 \mathrm{SS}, 20 \% \mathrm{CW}$ \\
\hline OD & $0.584 \mathrm{~cm}$ \\
\hline Thickness & $0.038 \mathrm{~cm}$ \\
\hline
\end{tabular}

\begin{tabular}{|l|l|}
\hline PRE-IRRADIATION CHARACTERISTICS \\
\hline BU (peak axial) & 4.2 at\% of HM \\
\hline Peak linear power & $\begin{array}{l}30 \mathrm{~kW} / \mathrm{m} \text { (17.1 } \mathrm{kW} / \mathrm{m} \\
\text { burnup-averaged) }\end{array}$ \\
\hline $\begin{array}{l}\text { Peak internal clad } \\
\text { temperature }\end{array}$ & (not available) \\
\hline $\begin{array}{l}\text { Fluence } \\
\text { (spectrum) }\end{array}$ & $\begin{array}{l}\text { Fluence not available; fast } \\
\text { spectrum }\end{array}$ \\
\hline
\end{tabular}

\section{OTHER FUEL SAMPLE INFORMATION}

Fuel $159.1 \mathrm{~g} /$ pin; breeder pellets natural $\mathrm{UO}_{2}$ (10 $\mathrm{cm}$ upper/solid, $46 \mathrm{~cm}$ lower/annular); bottom plenum $15.4 \mathrm{~cm}^{3}$ at $4.19 \mathrm{~atm}$ (cold).

As-irradiated fuel: low-power structure, $0.9 \mathrm{~mm}$ central hole, total fission gas release $1.61 \times 10^{-3}$ moles $=29 \%$.

\begin{tabular}{|l|l|}
\hline \multicolumn{2}{|c|}{ TEST CONDITIONS, MEASUREMENTS, \& ANALYSES } \\
\hline $\begin{array}{l}\text { Coolant } \\
\text { environment }\end{array}$ & $\begin{array}{l}\text { Flowing sodium at } 120 \mathrm{~g} / \mathrm{s} ; 0.47 \mathrm{MPa} \text { system } \\
\text { pressure, } 670 \mathrm{~K}\end{array}$ \\
\hline $\begin{array}{l}\text { Transient } \\
\text { conditions }\end{array}$ & $\begin{array}{l}\text { Fast rise to } 44 \mathrm{~kW} / \mathrm{m} \text { immediately followed by } \\
\text { a 15-s period power increase; scram } \\
\text { immediately upon pin fail ure }\end{array}$ \\
\hline $\begin{array}{l}\text { Fuel sample } \\
\text { configuration } \\
\text { in the test }\end{array}$ & Single fuel pin \\
\hline $\begin{array}{l}\text { Test vehicle \& } \\
\text { experiment } \\
\text { system }\end{array}$ & Mark IIIA Mod 1 loop with Single-Pin Test Train \\
\hline $\begin{array}{l}\text { Measurements } \\
\text { /diagnostics } \\
\text { during test }\end{array}$ & $\begin{array}{l}\text { Coolant temperature, flow rate, fuel movement } \\
\text { (by hodoscope) }\end{array}$ \\
\hline $\begin{array}{l}\text { Posttest } \\
\text { coolant flow } \\
\text { condition }\end{array}$ & Nearly the same as the pre-test flow rate. \\
\hline $\begin{array}{l}\text { Posttest } \\
\text { condition of } \\
\text { test sample(s) }\end{array}$ & $\begin{array}{l}\text { Cladding intact up to fuel height x/L 0.98. Local } \\
\text { breach at fuel top. Fuel dispersal from cladding. } \\
\text { most of fuel region. }\end{array}$ \\
\hline $\begin{array}{l}\text { Posttest } \\
\text { measurements } \\
\& \text { information }\end{array}$ & $\begin{array}{l}\text { Optical microscopy, neutron radiography, } \\
\text { photography, } \mathrm{x} \text { - radiography, autoradiography }\end{array}$ \\
\hline $\begin{array}{l}\text { Posttest } \\
\text { analyses }\end{array}$ & Thermal-mechanical analysis of fuel pin \\
\hline
\end{tabular}

\footnotetext{
KEY SUMMARY INFORMATION ABOUT THE TEST

Test $\mathrm{CO} 4$ was performed to characterize the failure behavior of irradiated, low-power-structure, bottom-plenum fuel pins when subjected to unprotected slow transient overpower (10 cents/s reactivity insertion) conditions beginning at a high power of $44 \mathrm{~kW} / \mathrm{m}$. The fuel pin failed at the top of the fuel region at about $123 \mathrm{~kW} / \mathrm{m}$ axial peak power. About $44 \%$ of the fuel was expelled from the pin. Fuel was expelled from regions along most of the original fuel zone, according to the melt radius of the fuel within the cladding. The flowtube was breached during the failure event, which substantially increased the flow area. Post-failure fuel motion was dispersive, resulting in a reactivity worth decrease equivalent to $~ 30 \%$ in a large fast reactor. Cladding strain was measured over most of the fuel region and was about $0.050 \%$ at a fuel height of $x / L=0.87$. The sevenpin bundle counterpart to this test is test LO3.
} 


\section{Summary of TREAT Test CO5}

Conducted 2 December 1982

Transient No. 2405

\begin{tabular}{l}
\hline PRE-TEST SAMPLE CHARACTERIZATION \\
\begin{tabular}{|l|l|}
\hline \multicolumn{2}{|c|}{ FUEL } \\
\hline Form & Helium-bonded MOX \\
\hline Composition & $\begin{array}{l}73 \mathrm{wt} \% \mathrm{UO}_{2}(23.5 \% \text { enriched), } \\
27 \mathrm{w} \% \mathrm{PuO}_{2}\end{array}$ \\
\hline Geometry & Annular pellets with flat ends \\
\hline Fissile height & $91.4 \mathrm{~cm}$ \\
\hline $\begin{array}{l}\text { Smear } \\
\text { density }\end{array}$ & $81 \%$ of theoretical \\
\hline
\end{tabular}
\end{tabular}

\begin{tabular}{|l|l|}
\hline \multicolumn{2}{|c|}{ CLADDING } \\
\hline Material & $316 \mathrm{SS}, 20 \% \mathrm{CW}$ \\
\hline OD & $0.584 \mathrm{~cm}$ \\
\hline Thickness & $0.038 \mathrm{~cm}$ \\
\hline
\end{tabular}

\begin{tabular}{|l|l|}
\hline PRE-IRRADIATION CHARACTERISTICS \\
\hline BU (peak axial) & 9 at\% of HM \\
\hline Peak linear power & $\begin{array}{l}54 \mathrm{~kW} / \mathrm{m} \\
(27.7 \mathrm{~kW} / \mathrm{m} \text { burnup- } \\
\text { averaged) }\end{array}$ \\
\hline $\begin{array}{l}\text { Peak internal clad } \\
\text { temperature }\end{array}$ & (not available) \\
\hline $\begin{array}{l}\text { Fluence } \\
\text { (spectrum) }\end{array}$ & $\begin{array}{l}\text { Fluence not available; fast } \\
\text { spectrum }\end{array}$ \\
\hline
\end{tabular}

\section{OTHER FUEL SAMPLE INFORMATION}

Fuel $166.6 \mathrm{~g}$; breeder pellets natural $\mathrm{UO}_{2}(10 \mathrm{~cm}$ upper/solid, $46 \mathrm{~cm}$ lower/annular); bottom plenum 15.4 $\mathrm{cm}^{3}$ at $4.19 \mathrm{~atm}$ (cold).

As-irradiated fuel: medium-to-high-power structure, similar overall gas retention as $4 \%$ burnup fuel in test CO4.

\begin{tabular}{|l|l|}
\hline \multicolumn{2}{|c|}{ TEST CONDITIONS, MEASUREMENTS, \& ANALYSES } \\
\hline $\begin{array}{l}\text { Coolant } \\
\text { environment }\end{array}$ & $\begin{array}{l}\text { Flowing sodium at } 120 \mathrm{~g} / \mathrm{s} ; 0.33 \mathrm{MPa} \text { system } \\
\text { pressure }\end{array}$ \\
\hline $\begin{array}{l}\text { Transient } \\
\text { conditions }\end{array}$ & $\begin{array}{l}\text { Fast rise to } 44 \mathrm{~kW} / \mathrm{m} \text { immediately followed by a } \\
\text { upon pin failure }\end{array}$ \\
\hline $\begin{array}{l}\text { Fuel sample } \\
\text { configuration } \\
\text { in the test }\end{array}$ & Single fuel pin \\
\hline $\begin{array}{l}\text { Test vehicle \& } \\
\text { experiment } \\
\text { system }\end{array}$ & Mark IlIA Mod 1 Loop with Single-Pin Test Train \\
\hline $\begin{array}{l}\text { Measurements } \\
\text { /diagnostics } \\
\text { during test }\end{array}$ & $\begin{array}{l}\text { Coolant temperature, flow rate, fuel movement } \\
\text { (by hodoscope) }\end{array}$ \\
\hline $\begin{array}{l}\text { Posttest } \\
\text { coolant flow } \\
\text { condition }\end{array}$ & Fully-blocked flow channel \\
\hline $\begin{array}{l}\text { Posttest } \\
\text { condition of } \\
\text { test sample(s) }\end{array}$ & $\begin{array}{l}\text { Localized cladding breach. Fuel dispersal from } \\
\text { cladding. Unmelted fuel on interior wall of } \\
\text { cladding along most of fuel region. }\end{array}$ \\
\hline $\begin{array}{l}\text { Posttest } \\
\text { measurements } \\
\text { \& information }\end{array}$ & $\begin{array}{l}\text { Optical microscopy, neutron radiography, } \\
\text { photography, } \mathrm{x} \text {-radiography, autoradiography }\end{array}$ \\
\hline $\begin{array}{l}\text { Posttest } \\
\text { analyses }\end{array}$ & Thermal-mechanical analysis of fuel pin. \\
\hline
\end{tabular}

\section{KEY SUMMARY INFORMATION ABOUT THE TEST}

Test CO5 was performed to characterize the failure behavior of irradiated, medium-to-high-power microstructure, bottomplenum fuel pins when subjected to unprotected slow TOP (10 cents/s reactivity insertion) conditions beginning at a high power level of $44 \mathrm{~kW} / \mathrm{m}$. The fuel expanded axially $\sim 11 \mathrm{~mm}$ prior to cladding failure. The fuel pin failed at the top of the fuel region at about $131 \mathrm{~kW} / \mathrm{m}$ axial peak power. About $33 \%$ of the fuel was expelled from the pin. Fuel was expelled from regions along most of the original fuel zone, according to the melt radius of the fuel within the cladding. Post-failure fuel motion was dispersive, resulting in a reactivity worth decrease equivalent to $\sim 15 \%$ in a large fast reactor. Cladding strain was measured over most of the fuel region and was about $0.37 \%$ at a fuel height of $x / L=0.87$. 


\section{Summary of TREAT Test CO6R \\ Conducted 20 July 1983}

Transient No. 2467

\begin{tabular}{l}
\hline PRE-TEST SAMPLE CHARACTERIZATION \\
\begin{tabular}{|l|l|}
\hline \multicolumn{2}{|c|}{ FUEL } \\
\hline Form & Helium-bonded MOX \\
\hline Composition & $\begin{array}{l}\mathrm{UO}_{2} \text { (natural U) }-31.5 w^{2} \% \mathrm{PuO}_{2} \\
\text { Oxygen-to-metal ratio }=1.985\end{array}$ \\
\hline Geometry & Annular pellets with flat ends \\
\hline Fissile height & $91.4 \mathrm{~cm}$ \\
\hline $\begin{array}{l}\text { Smear } \\
\text { density }\end{array}$ & $81 \%$ of theoretical \\
\hline
\end{tabular}
\end{tabular}

\begin{tabular}{|l|l|}
\hline \multicolumn{2}{|c|}{ CLADDING } \\
\hline Material & M316 stainless steel \\
\hline OD & $0.585 \mathrm{~cm}$ \\
\hline Thickness & $0.077 \mathrm{~cm}$ \\
\hline
\end{tabular}

\begin{tabular}{|l|l|}
\hline PRE-IRRADIATION CHARACTERISTICS \\
\hline BU (peak axial) & 4.2 at\% of HM \\
\hline Peak linear power & $\sim 30 \mathrm{~kW} / \mathrm{m}$ \\
\hline $\begin{array}{l}\text { Peak internal clad } \\
\text { temperature }\end{array}$ & (not available) \\
\hline $\begin{array}{l}\text { Fluence } \\
\text { (spectrum) }\end{array}$ & $\begin{array}{l}\text { Fluence not available; fast } \\
\text { spectrum }\end{array}$ \\
\hline
\end{tabular}

\begin{tabular}{|c|c|}
\hline \multicolumn{2}{|c|}{ TEST CONDITIONS, MEASUREMENTS, \& ANALYSES } \\
\hline $\begin{array}{l}\text { Coolant } \\
\text { environment }\end{array}$ & $\begin{array}{l}\text { Flowing sodium, inlet temperature } 670 \mathrm{~K}, 0.184 \\
\text { MPa system pressure }\end{array}$ \\
\hline $\begin{array}{l}\text { Transient } \\
\text { conditions }\end{array}$ & $\begin{array}{l}\text { Sub-nominal initial flow rate, several seconds at } \\
\sim 22 \mathrm{~kW} / \mathrm{m} \text {, then two-stage power burst, first } \\
\text { increasing power by } 3 \mathrm{x} \text { then burst of } \sim 0.40 \mathrm{~s} \\
\text { FWHM increasing power by another factor of } 18 \text {. } \\
\text { Peak power } \sim 1300 \mathrm{~kW} / \mathrm{m} \text {. }\end{array}$ \\
\hline $\begin{array}{l}\text { Fuel sample } \\
\text { configuration } \\
\text { in the test }\end{array}$ & Single pin \\
\hline $\begin{array}{l}\text { Test vehicle \& } \\
\text { experiment } \\
\text { system }\end{array}$ & Single-Pin Test Loop \\
\hline $\begin{array}{l}\text { Measurements } \\
\text { /diagnostics } \\
\text { during test }\end{array}$ & $\begin{array}{l}\text { Coolant temperature, coolant flow rate, coolant } \\
\text { pressure, fuel distribution }\end{array}$ \\
\hline $\begin{array}{l}\text { Posttest } \\
\text { coolant flow } \\
\text { condition }\end{array}$ & $\begin{array}{l}\text { (not relevant to the accident scenario due to the } \\
\text { large amount of excess energy generated) }\end{array}$ \\
\hline $\begin{array}{l}\text { Posttest } \\
\text { condition of } \\
\text { test sample(s) }\end{array}$ & Gross disruption \\
\hline $\begin{array}{l}\text { Posttest } \\
\text { measurements } \\
\text { \& information }\end{array}$ & Neutron radiography \\
\hline $\begin{array}{l}\text { Posttest } \\
\text { analyses }\end{array}$ & $\begin{array}{l}\text { Fuel-pin thermal-mechanics, coolant thermal- } \\
\text { hydraulics, fuel motion }\end{array}$ \\
\hline
\end{tabular}

\section{OT HER FUEL SAMPLE INFORMATION}

Fuel $159.1 \mathrm{~g} / \mathrm{pin}$; breeder pellets natural $\mathrm{UO}_{2}(10 \mathrm{~cm}$ upper/solid, $46 \mathrm{~cm}$ lower/annular); plenum $15.4 \mathrm{~cm}^{3}$ at $4.19 \mathrm{~atm}$ (cold).

As-irradiated fuel: low-power structure, $0.9 \mathrm{~mm}$ central

hole, total fission gas release $1.61 \times 10^{-3}$ moles $=29 \%$.

\section{KEY SUMMARY INFORMATION ABOUT THE TEST}

The objective of test CO6R was to determine the time and location of fuel-pin failure during a type of hypothetical transientunder-cooling-driven overpower (TUCOP) accident. Fuel axial expansion $(\sim 5 \mathrm{~mm})$ and some coolant voiding occurred prior to fuel pin failure. Cladding failed at approximately $960 \mathrm{~kW} / \mathrm{m}$ at a relative fuel column height of 0.79 , as the power was still rising. More than half of the burst energy was generated after the cladding failed, resulting in gross disruption of cladding and fuel. Post-failure dispersive fuel motion was equivalent to a reactivity worth reduction of $\sim 18 \%$ in a large fast reactor. The seven-pin counterpart to this experiment was test LO7. 


\section{Appendix D: One-page Summaries of LO-series Tests}

One-page summaries of each of the PFR/TREAT CO- and LO-series tests are included here. These summaries can also be found in TREXR [6] 


\section{Summary of TREAT Test L01 \\ Conducted 24 November 1980}

Transient No. 2246

PRE-TEST SAMPLE CHARACTERIZATION
\begin{tabular}{|l|l|}
\hline \multicolumn{2}{|c|}{ FUEL } \\
\hline Form & He-bonded MOX \\
\hline Composition & $\begin{array}{l}\text { UO2 (natural U)-PuO2; } \\
\text { Pul/(U+Pu) }=0.289 \\
\text { Oxygen-to-metal ratio 1.965 }\end{array}$ \\
\hline Geometry & Annular pellet, flat ends \\
\hline Fissile height & $91.4 \mathrm{~cm}$ \\
\hline $\begin{array}{l}\text { Smear } \\
\text { density }\end{array}$ & $78.5 \%$ of theoretical \\
\hline
\end{tabular}

\begin{tabular}{|l|l|}
\hline \multicolumn{2}{|c|}{ CLADDING } \\
\hline Material & SS316 20\% CW \\
\hline OD & $0.584 \mathrm{~cm}$ \\
\hline Thickness & $0.038 \mathrm{~cm}$ \\
\hline
\end{tabular}

\begin{tabular}{|l|l|}
\hline \multicolumn{2}{|c|}{ TEST CONDITIONS, MEASUREMENTS, \& ANALYSES } \\
\hline $\begin{array}{l}\text { Coolant } \\
\text { environment }\end{array}$ & Flowing sodium \\
\hline $\begin{array}{l}\text { Transient } \\
\text { conditions }\end{array}$ & $\begin{array}{l}\text { Steady power for } ~ 4 \mathrm{~s} \text { ( } ~ 31 \mathrm{~kW} / \mathrm{m} \text { at midheight } \\
\text { of hottest pin), then power burst peaking at } \\
\sim 1000 \mathrm{~kW} / \mathrm{m} \text { in } 0.79 \mathrm{~s}, \text { then scram }\end{array}$ \\
\hline $\begin{array}{l}\text { Fuel sample } \\
\text { configuration } \\
\text { in the test }\end{array}$ & 7-pin, grid-spaced bunde \\
\hline $\begin{array}{l}\text { Test vehicle \& } \\
\text { experiment } \\
\text { system }\end{array}$ & $\begin{array}{l}\text { Mark IIIA loop, dysprosium axial shaping collar, } \\
\text { BSi \& dysprosium neutron filters }\end{array}$ \\
\hline $\begin{array}{l}\text { Measurements } \\
\text { /diagnostics } \\
\text { during test }\end{array}$ & $\begin{array}{l}\text { Flowmeters, pressure transducers, } \\
\text { thermocouples, fuel motion (hodoscope) }\end{array}$ \\
\hline $\begin{array}{l}\text { Posttest } \\
\text { coolant flow } \\
\text { condition }\end{array}$ & $\begin{array}{l}\text { Complete upper blockage of once-molten fuel; } \\
\text { metal blockage at the bottom of the initial fuel } \\
\text { region }\end{array}$ \\
\hline $\begin{array}{l}\text { Posttest } \\
\text { condition of } \\
\text { test sample(s) }\end{array}$ & $\begin{array}{l}\text { Severe cladding failure and dispersive fuel } \\
\text { motion, complete pin disruption in upper two- } \\
\text { thirds of fuel zone }\end{array}$ \\
\hline $\begin{array}{l}\text { Posttest } \\
\text { measurements } \\
\text { \& information }\end{array}$ & $\begin{array}{l}\text { Macro- and micro examination, neutron } \\
\text { radiography, metallographic examination, sibling } \\
\text { pin analysis, }\end{array}$ \\
\hline $\begin{array}{l}\text { Posttest } \\
\text { analyses }\end{array}$ & $\begin{array}{l}\text { Thermal hydraulic, cladding failure, fuel and } \\
\text { cladding motion, code validation }\end{array}$ \\
\hline
\end{tabular}

U.S.-mfg. PFR-like pins; $163 \mathrm{~g} /$ pin fuel mass:

Central hole in fuel: $0.165 \mathrm{~cm}$ diameter;

Annular breeder pellets of natural UO2 $(9.4 \mathrm{~cm}$ upper, 46 $\mathrm{cm}$ lower);

$15.4 \mathrm{~cm} 3$ bottom plenum at $4.19 \mathrm{~atm}$ (cold)

\begin{tabular}{l}
\hline KEY SUMMARY INFORMATION ABOUT THE TEST \\
\hline Test LO1 subjected fresh MOX fuel to a simulation of a $\$ 5 / \mathrm{s}$ transient overpower accident. No prefailure axial fuel expansion \\
or fuel motion occurred, within the sensitivity limit (about $+/-6 \mathrm{~mm}$ ) of the hodoscope. There was severe cladding failure with \\
complete pin disruption in the upper two-thirds of the fuel region. Fuel released into the coolant channel was relocated overall \\
upward, with its dispersal corresponding to a net loss of reactivity worth of about $10 \%$ in a large fast reactor. The captor grid \\
in the flow channel above the fuel pins presented a significant impediment to fuel motion as well as a fuel collection site. The \\
posttest material configuration included a complete upper blockage by once-molten fuel and a metal blockage at the lower \\
end of the fuel region. It was concluded that cladding failed by melt-through. In comparison with a companion test (LO2) on \\
pre-irradiated fuel, the failure in LO1 occurred much later, and less fuel disruption resulted. The reactivity loss from fuel \\
disruption was only slightly more than in LO2.
\end{tabular}




\section{Summary of TREAT Test LO2

\author{
Conducted 5 March 1982
}

Transient No. 2327

\section{PRE-TEST SAMPLE CHARACTERIZATION}

\begin{tabular}{|l|l|}
\hline \multicolumn{2}{|c|}{ FUEL } \\
\hline Form & He-bonded MOX \\
\hline Composition & $\begin{array}{l}\text { UO2 (natural U)-PuO2; } \\
\text { Pu/(U+Pu) = 0.315 } \\
\text { Oxygen-to-metal ratio 1.985 }\end{array}$ \\
\hline Geometry & Annular pellets with flat ends \\
\hline Fissile height & $91.4 \mathrm{~cm}$ \\
\hline $\begin{array}{l}\text { Smear } \\
\text { density }\end{array}$ & $79 \%$ of theoretical \\
\hline
\end{tabular}

\begin{tabular}{|l|l|}
\hline \multicolumn{2}{|c|}{ CLADDING } \\
\hline Material & SS316 20\% CW \\
\hline OD & $0.584 \mathrm{~cm}$ \\
\hline Thickness & $0.038 \mathrm{~cm}$ \\
\hline
\end{tabular}

PRE-IRRADIATION CHARACTERISTICS

\begin{tabular}{|l|l|}
\hline BU (peak axial) & 4.2 at\% of $\mathrm{HM}$ \\
\hline Peak linear power & $\sim 30 \mathrm{~kW} / \mathrm{m}$ equivalent \\
\hline $\begin{array}{l}\text { Peak internal clad } \\
\text { temperature }\end{array}$ & (not available) \\
\hline $\begin{array}{l}\text { Fluence } \\
\text { (spectrum) }\end{array}$ & $\begin{array}{l}\text { Fluence not available; fast } \\
\text { spectrum }\end{array}$ \\
\hline
\end{tabular}

\section{OTHER FUEL SAMPLE INFORMATION}

Prototypic PFR fuel pins; $159.1 \mathrm{~g} /$ pin fuel mass :

Annular breeder pellets of natural $\mathrm{UO}_{2}(10 \mathrm{~cm}$ upper, 46 $\mathrm{cm}$ lower); $15.4 \mathrm{~cm}^{3}$ bottom plenum at $4.19 \mathrm{~atm}$ (cold) ;

As-irradiated low-power structure, $0.09 \mathrm{~cm}$ central hole,

$1.61 \times 10^{-3}$ moles total fission gas release $(29 \%$ of gas

generated).

\begin{tabular}{|l|l|}
\hline \multicolumn{2}{|c|}{ TEST CONDITIONS, MEASUREMENTS, \& ANALYSES } \\
\hline $\begin{array}{l}\text { Coolant } \\
\text { environment }\end{array}$ & Flowing sodium \\
\hline $\begin{array}{l}\text { Transient } \\
\text { conditions }\end{array}$ & $\begin{array}{l}\text { Steady power for } 4.1 \mathrm{~s} \text { ( } ~ 28 \mathrm{~kW} / \mathrm{m} \text { at the } \\
\text { midheight of the hottest pin), then power burst } \\
\text { peaking at } \sim 1000 \mathrm{~kW} / \mathrm{m} \text { in } 0.73 \mathrm{~s} \text {, then scram }\end{array}$ \\
\hline $\begin{array}{l}\text { Fuel sample } \\
\text { configuration } \\
\text { in the test }\end{array}$ & 7-pin bundle; grid-spaced pins \\
\hline $\begin{array}{l}\text { Test vehicle \& } \\
\text { experiment } \\
\text { system }\end{array}$ & $\begin{array}{l}\text { Mark IIIA Loop, dysprosium axial shaping collar, } \\
\text { B } \text { Si and dysprosium neutron filters }\end{array}$ \\
\hline $\begin{array}{l}\text { Measurements } \\
\text { /diagnostics } \\
\text { during test }\end{array}$ & $\begin{array}{l}\text { Flow rate, coolant pressure, temperatures, fuel } \\
\text { distribution }\end{array}$ \\
\hline $\begin{array}{l}\text { Posttest } \\
\text { coolant flow } \\
\text { condition }\end{array}$ & $\begin{array}{l}\text { Complete upper blockage of once-molten } \\
\text { fuel/cladding material }\end{array}$ \\
\hline $\begin{array}{l}\text { Posttest } \\
\text { condition of } \\
\text { test sample(s) }\end{array}$ & $\begin{array}{l}\text { Severe cladding failure, net upward dispersive } \\
\text { fuel motion, complete pin disruption in upper } 3 / 4 \\
\text { of fuel zone }\end{array}$ \\
\hline $\begin{array}{l}\text { Posttest } \\
\text { measurements } \\
\text { \& information }\end{array}$ & $\begin{array}{l}\text { Macro and micro examination, neutron } \\
\text { radiography, metallographic examination }\end{array}$ \\
\hline $\begin{array}{l}\text { Posttest } \\
\text { analyses }\end{array}$ & $\begin{array}{l}\text { Thermo-hydraulic analysis, fuel and cladding } \\
\text { motion analysis, code validation }\end{array}$ \\
\hline
\end{tabular}

\section{KEY SUMMARY INFORMATION ABOUT THE TEST}

Test LO2 subjected fresh MOX fuel to a simulation of a $\$ 5 / \mathrm{s}$ transient overpower accident. It was a counterpart to fresh-fuel test LO1, but using moderate-burnup fuel. No prefailure axial fuel expansion or fuel motion occurred, within sensitivity limit (about $+/-6 \mathrm{~mm}$ ) of the hodoscope. The cladding failed, apparently by a rapid pressurization due to fission gas release from the fuel (much earlier than the fuel pins failed in LO1). There was complete pin disruption in the upper three-fourths of the fuel region, and fuel loss was mainly from the bottom half of the fuel region. As in LO1, the fuel motion was net upward. The degree of cladding failure and extent of fuel disruption in LO2 was greater than in LO1, but the reactivity loss due to fuel dispersal occurred more slowly and was slightly less than the $10 \%$ that occurred in LO1. The upper captor grid presented a significant restraint to post-failure motion of molten fuel. By the end of the test, the fuel region has become blocked at the top and bottom by solidified metal. 


\section{Summary of TREAT Test LO3

\author{
Conducted 5 May 1982
}

Transient No. 2342

\section{PRE-TEST SAMPLE CHARACTERIZATION}

\begin{tabular}{|l|l|}
\hline \multicolumn{2}{|c|}{ FUEL } \\
\hline Form & He-bonded MOX \\
\hline Composition & $\begin{array}{l}\mathrm{UO2} \text { (natural U)-PuO2; } \\
\mathrm{Pu} /(\mathrm{U}+\mathrm{Pu})=0.315 \\
\text { Oxygen-to-metal ratio 1.980 }\end{array}$ \\
\hline Geometry & Annular pellets with flat ends \\
\hline Fissile height & $91.4 \mathrm{~cm}$ \\
\hline $\begin{array}{l}\text { Smear } \\
\text { density }\end{array}$ & $80 \%$ of theoretical \\
\hline
\end{tabular}

\begin{tabular}{|l|l|}
\hline \multicolumn{2}{|c|}{ CLADDING } \\
\hline Material & SS316 20\%CW \\
\hline OD & $0.584 \mathrm{~cm}$ \\
\hline Thickness & $0.038 \mathrm{~cm}$ \\
\hline
\end{tabular}

PRE-IRRADIATION CHARACTERISTICS

\begin{tabular}{|l|l|}
\hline BU (peak axial) & 4.2 at\% of $\mathrm{HM}$ \\
\hline Peak linear power & $\sim 30 \mathrm{~kW} / \mathrm{m}$ equivalent \\
\hline $\begin{array}{l}\text { Peak internal clad } \\
\text { temperature }\end{array}$ & (not available) \\
\hline $\begin{array}{l}\text { Fluence } \\
\text { (spectrum) }\end{array}$ & $\begin{array}{l}\text { Fluence not available; fast } \\
\text { spectrum }\end{array}$ \\
\hline
\end{tabular}

\section{OTHER FUEL SAMPLE INFORMATION}

Prototypic PFR fuel pins; $159.1 \mathrm{~g} /$ pin fuel mass :

Annular breeder pellets of natural $\mathrm{UO}_{2}(10 \mathrm{~cm}$ upper, 46

$\mathrm{cm}$ lower); $15.4 \mathrm{~cm}^{3}$ bottom plenum at $4.19 \mathrm{~atm}$ (cold) ;

As-irradiated low-power structure, $0.09 \mathrm{~cm}$ central hole,

$1.61 \times 10^{-3}$ moles total fission gas release $(29 \%$ of gas

generated).

\begin{tabular}{|l|l|}
\hline \multicolumn{2}{|c|}{ TEST CONDITIONS, MEASUREMENTS, \& ANALYSES } \\
\hline $\begin{array}{l}\text { Coolant } \\
\text { environment }\end{array}$ & Flowing sodium \\
\hline $\begin{array}{l}\text { Transient } \\
\text { conditions }\end{array}$ & $\begin{array}{l}\text { Rapid power rise first to 71 } \mathrm{kW} / \mathrm{m} \text { at midplane of } \\
\text { hettest pin; then further increase on 14-s period. } \\
\text { 122 kW/m), continuing for 2.5 slonger on 14-s } \\
\text { period generating 46\% more reactor energy than } \\
\text { planned. }\end{array}$ \\
\hline $\begin{array}{l}\text { Fuel sample } \\
\text { configuration } \\
\text { in the test }\end{array}$ & 7-pin hexagonal bundle; grid-spaced pins \\
\hline $\begin{array}{l}\text { Test vehicle \& } \\
\text { experiment } \\
\text { system }\end{array}$ & $\begin{array}{l}\text { Modified Mark IIIA loop with dual ALIPs; } \\
\text { dysprosium shaping collars }\end{array}$ \\
\hline $\begin{array}{l}\text { Measurements } \\
\text { /diagnostics } \\
\text { during test }\end{array}$ & $\begin{array}{l}\text { Flowmeters, pressure transducers, } \\
\text { thermocouples, fuel motion (hodoscope) }\end{array}$ \\
\hline $\begin{array}{l}\text { Posttest } \\
\text { coolant flow } \\
\text { condition }\end{array}$ & $\begin{array}{l}\text { Complete blockage prompty upon initial pin } \\
\text { failure which then melted as the extra energy } \\
\text { was generated. Final blockage at top of sodium } \\
\text { pump. }\end{array}$ \\
\hline $\begin{array}{l}\text { Posttest } \\
\text { condition of } \\
\text { test sample(s) }\end{array}$ & $\begin{array}{l}\text { Extremely severe cladding and fuel relocation } \\
\text { (gross disruption), molten fuel and steel } \\
\text { relocation throughoutloop components }\end{array}$ \\
\hline $\begin{array}{l}\text { Posttest } \\
\text { measurements } \\
\text { \& information }\end{array}$ & $\begin{array}{l}\text { Macro and micro examination, neutron } \\
\text { radiography, metallographic examination, fuel } \\
\text { and cladding distribution }\end{array}$ \\
\hline $\begin{array}{l}\text { Posttest } \\
\text { analyses }\end{array}$ & $\begin{array}{l}\text { Pin failure, thermal-hydraulics, post-failure fuel } \\
\text { motion, code validation }\end{array}$ \\
\hline
\end{tabular}

\section{KEY SUMMARY INFORMATION ABOUT THE TEST}

Test LO3 was conducted to investigate the effects of a slow transient overpower accident on UK Prototype Fast Reactor (PFR) pin failure and fuel motion. Cladding failure was caused by the internal cavity pressurization resulting from fuel expansion and fission gas release and occurred at a fuel height $x / L=\sim 0.8$. Fuel dispersion soon after cladding failure corresponded to a reactivity loss of about $6 \%$ in a large fast reactor. A software error prevented the planned scram from occurring upon initial pin failure and led to continued fuel heating and resulting fuel and cladding movement. Although it was predicted that the oxide fuel would limit dispersal by solidifying to form coolant channel blockages, the severe overheat allowed for the re-melting and movement of fuel. This molten fuel tended to collect within the ALIP sodium pump inlet, causing damage to the experimental hardware. 


\section{Summary of TREAT Test LO4 \\ Conducted 17 February 1983 \\ Transient No. 2422}

\section{PRE-TEST SAMPLE CHARACTERIZATION}

\begin{tabular}{|l|l|}
\hline \multicolumn{2}{|c|}{ FUEL } \\
\hline Form & He-bonded MOX \\
\hline Composition & $\begin{array}{l}\text { UO2 (natural U)-PuO2; } \\
\text { Pu/(U+Pu) }=0.315 \\
\text { Oxygen-to-metal ratio 1.985 }\end{array}$ \\
\hline Geometry & Annular pellets with flat ends \\
\hline Fissile height & $91.4 \mathrm{~cm}$ \\
\hline $\begin{array}{l}\text { Smear } \\
\text { density }\end{array}$ & $79 \%$ of theoretical density \\
\hline
\end{tabular}

\begin{tabular}{|l|l|}
\hline \multicolumn{2}{|c|}{ CLADDING } \\
\hline Material & SS316 20\% CW \\
\hline OD & $0.584 \mathrm{~cm}$ \\
\hline Thickness & $0.038 \mathrm{~cm}$ \\
\hline
\end{tabular}

\begin{tabular}{|l|l|}
\hline \multicolumn{2}{|l|}{ PRE-IRRADIATION CHARACTERISTICS } \\
\hline BU (peak axial) & 4.12 at\% of HM \\
\hline Peak linear power & $\sim 30 \mathrm{~kW} / \mathrm{m}$ equivalent \\
\hline $\begin{array}{l}\text { Peak internal clad } \\
\text { temperature }\end{array}$ & (not available) \\
\hline $\begin{array}{l}\text { Fluence } \\
\text { (spectrum) }\end{array}$ & $\begin{array}{l}\text { Fluence not available; fast } \\
\text { spectrum }\end{array}$ \\
\hline
\end{tabular}

\section{OTHER FUEL SAMPLE INFORMATION}

Prototypic PFR fuel pins; $159.1 \mathrm{~g} /$ pin fuel mass ;

Annular breeder pellets of natural $\mathrm{UO}_{2}(10 \mathrm{~cm}$ upper, 46 $\mathrm{cm}$ lower); $15.4 \mathrm{~cm}^{3}$ bottom plenum at $4.19 \mathrm{~atm}$ (cold) ;

As-irradiated low-power structure, $0.09 \mathrm{~cm}$ central hole,

$1.61 \times 10^{-3}$ moles total fission gas release $(29 \%$ of gas generated).

\begin{tabular}{|l|l|}
\hline \multicolumn{2}{|c|}{ TEST CONDITIONS, MEASUREMENTS, \& ANALYSES } \\
\hline $\begin{array}{l}\text { Coolant } \\
\text { environment }\end{array}$ & Flowing sodium, initially at 30\% of nominal \\
\hline $\begin{array}{l}\text { Transient } \\
\text { conditions }\end{array}$ & $\begin{array}{l}\text { Several seconds at } 44 \mathrm{~kW} / \mathrm{m} \text { (average pin at the } \\
\text { midplane) with flow reduction by } 50 \% \text { in } 5 \mathrm{~s} \\
\text { inducing boiling and triggering power rise with } \\
0.44 \text { s period for one e-fold, then rise on } ~ 0.18 \mathrm{~s} \\
\text { period reaching 920 kW/m in } ~ 0.35 \mathrm{~s} \text {, then } \\
\text { scram. Total energy } ~ 1500 \mathrm{~kW} / \mathrm{kg} \text { in average } \\
\text { pin at midplane. }\end{array}$ \\
\hline $\begin{array}{l}\text { Fuel sample } \\
\text { configuration } \\
\text { in the test }\end{array}$ & 7-pin hexagonal bundle; grid-spaced pins \\
\hline $\begin{array}{l}\text { Test vehicle \& } \\
\text { experiment } \\
\text { system }\end{array}$ & Mark-IIIC loop; dysprosium shaping collars \\
\hline $\begin{array}{l}\text { Measurements } \\
\text { /diagnostics } \\
\text { during test }\end{array}$ & $\begin{array}{l}\text { Pressure transducers, flowmeters, } \\
\text { thermocouples, fuel motion (hodoscope) }\end{array}$ \\
\hline $\begin{array}{l}\text { Posttest } \\
\text { coolant flow } \\
\text { condition }\end{array}$ & $\begin{array}{l}\text { Total upper blockage of once-molten fuel and } \\
\text { steel around grid spacers, lower blockage of } \\
\text { once-molten steel }\end{array}$ \\
\hline $\begin{array}{l}\text { Posttest } \\
\text { condition of } \\
\text { test sample(s) }\end{array}$ & $\begin{array}{l}\text { Gross fuel and cladding disruption (severe } \\
\text { melting and relocation) }\end{array}$ \\
\hline $\begin{array}{l}\text { Posttest } \\
\text { measurements } \\
\& \text { information }\end{array}$ & $\begin{array}{l}\text { Neutron radiography, macro examination, fuel } \\
\text { and cladding distribution }\end{array}$ \\
\hline $\begin{array}{l}\text { Posttest } \\
\text { analyses }\end{array}$ & $\begin{array}{l}\text { Pin failure, thermal-hydraulics, post-failure fuel } \\
\text { motion, code validation }\end{array}$ \\
\hline
\end{tabular}

\section{KEY SUMMARY INFORMATION ABOUT THE TEST}

Test LO4 simulated high-power, lead-subassembly transient-undercooling-driven overpower (TUCOP) conditions by causing sodium voiding prior to the power burst. (The first part of the power burst was to simulate reactivity increase due to coolant voiding in lead channels in a large fast reactor; the second part of the burst was to simulate reactivity from voiding progression into assemblies of intermediate power.) Prior to cladding failure there was significant fuel expansion but no fuel motion. Pin failure occurred at fuel height $x / L=0.66$ with fuel escaping into mostly-voided channels when the cladding was weak or molten. This was followed by severe cladding meltthrough, gross fuel and cladding disruption, flowtube meltthrough, and dispersive fuel movement. A total upper blockage of once-molten fuel and steel formed around a grid spacer above the fuel region, and a lower blockage of once-molten steel filled most of the flow channel just below the bottom of the fuel region. The fuel motion corresponded to a net loss of approximately $14 \%$ reactivity worth in a large fast reactor. 


\section{Summary of TREAT Test LO5

\author{
Conducted 1 April 1983
}

Transient No. 2432

\section{PRE-TEST SAMPLE CHARACTERIZATION}

\begin{tabular}{|l|l|}
\hline \multicolumn{2}{|c|}{ FUEL } \\
\hline Form & He-bonded MOX \\
\hline Composition & $\begin{array}{l}\mathrm{UO2} \text { (natural U)-PuO2; } \\
\text { Pu/(U+Pu) }=0.315 \\
\text { Oxygen-to-metal ratio 1.985 }\end{array}$ \\
\hline Geometry & Annular pellets with flat ends \\
\hline Fissile height & $91.4 \mathrm{~cm}$ \\
\hline $\begin{array}{l}\text { Smear } \\
\text { density }\end{array}$ & $79 \%$ of theoretical density \\
\hline
\end{tabular}

\begin{tabular}{|l|l|}
\hline \multicolumn{2}{|c|}{ CLADDING } \\
\hline Material & SS316 20\%CW \\
\hline OD & $0.584 \mathrm{~cm}$ \\
\hline Thickness & $0.038 \mathrm{~cm}$ \\
\hline
\end{tabular}

PRE-IRRADIATION CHARACTERISTICS

\begin{tabular}{|l|l|}
\hline BU (peak axial) & 4.12 at\% of HM \\
\hline Peak linear power & $\sim 30 \mathrm{~kW} / \mathrm{m}$ equivalent \\
\hline $\begin{array}{l}\text { Peak internal clad } \\
\text { temperature }\end{array}$ & (not available) \\
\hline $\begin{array}{l}\text { Fluence } \\
\text { (spectrum) }\end{array}$ & $\begin{array}{l}\text { Fluence not available; fast } \\
\text { spectrum }\end{array}$ \\
\hline
\end{tabular}

\section{OTHER FUEL SAMPLE INFORMATION}

Prototypic PFR fuel pins; $159.1 \mathrm{~g} /$ pin fuel mass :

Annular breeder pellets of natural $\mathrm{UO}_{2}(10 \mathrm{~cm}$ upper, 46 $\mathrm{cm}$ lower); $15.4 \mathrm{~cm}^{3}$ bottom plenum at $4.19 \mathrm{~atm}$ (cold) ;

As-irradiated low-power structure, $0.09 \mathrm{~cm}$ central hole,

$1.61 \times 10^{-3}$ moles total fission gas release $(29 \%$ of gas

generated).

\begin{tabular}{|l|l|}
\hline \multicolumn{2}{|c|}{ TEST CONDITIONS, MEASUREMENTS, \& ANALYSES } \\
\hline $\begin{array}{l}\text { Coolant } \\
\text { environment }\end{array}$ & $\begin{array}{l}\text { Flowing sodium; flow rate held at 36\% of } \\
\text { nominal }\end{array}$ \\
\hline $\begin{array}{l}\text { Transient } \\
\text { conditions }\end{array}$ & $\begin{array}{l}\text { Several seconds at 44 kW/m (average pin at } \\
\text { midplane), then power rise with 0.44 s period for } \\
\text { one e-fold, then rise on 0.18 s period reaching } \\
\sim 920 \mathrm{~kW} / \mathrm{m} \text { in } ~ \\
\sim 1500 \mathrm{~s} \mathrm{~s}, \text { then scram. Total energy }\end{array}$ \\
\hline $\begin{array}{l}\text { Fuel sample } \\
\text { configuration } \\
\text { in the test }\end{array}$ & 7 -pin hexagonal bundle; grid-spaced pins \\
\hline $\begin{array}{l}\text { Test vehicle \& } \\
\text { experiment } \\
\text { system }\end{array}$ & Mark-IIIC loop, dysprosium shaping collars \\
\hline $\begin{array}{l}\text { Measurements } \\
\text { /diagnostics } \\
\text { during test }\end{array}$ & $\begin{array}{l}\text { Coolant channel flow rate and pressure, } \\
\text { temperatures, fuel motion (hodoscope) }\end{array}$ \\
\hline $\begin{array}{l}\text { Posttest } \\
\text { coolant flow } \\
\text { condition }\end{array}$ & $\begin{array}{l}\text { Total blockage of once-molten fuel and steel } \\
\text { around upper breeder region, lower blockage of } \\
\text { once-molten steel }\end{array}$ \\
\hline $\begin{array}{l}\text { Posttest } \\
\text { condition of } \\
\text { test sample(s) }\end{array}$ & $\begin{array}{l}\text { Gross fuel and cladding disruption (severe } \\
\text { melting and movement), , flowtube melt-through, } \\
\text { pellet stack collapse }\end{array}$ \\
\hline $\begin{array}{l}\text { Posttest } \\
\text { measurements } \\
\text { \& information }\end{array}$ & $\begin{array}{l}\text { Neutron radiography and tomography, macro } \\
\text { examination, fuel and cladding distribution }\end{array}$ \\
\hline $\begin{array}{l}\text { Posttest } \\
\text { analyses }\end{array}$ & $\begin{array}{l}\text { Pin failure, thermal-hydraulics, post-failure fuel } \\
\text { motion, code validation }\end{array}$ \\
\hline
\end{tabular}

\section{KEY SUMMARY INFORMATION ABOUT THE TEST}

Test LO5 simulated intermediate-power-subassembly conditions by causing the planned power burst to occur while the sodium coolant was just beginning to boil. (The first part of the power burst was to simulate reactivity increase due to coolant voiding in lead channels in a large fast reactor; the second part of the burst was to simulate reactivity from voiding progression into assemblies of intermediate power.) Prior to cladding failure there was significant fuel expansion but not fuel motion. Weakened cladding failed into two-phase sodium slightly below the fuel midplane, followed by severe cladding failure and dispersive fuel movement. This resulted first in a $7 \%$ reduction in reactivity worth but ending in a fuel distribution corresponding to an overall reactivity gain of $4 \%$ in a large fast reactor. By the end of the test, a total blockage of oncemolten fuel and steel at a grid spacer near the upper breeder region and a partial lower blockage of once-molten steel at bottom of the fuel region had developed. 


\section{Summary of TREAT Test LO6}

Conducted 9 September 1983

Transient No. 2486

\section{PRE-TEST SAMPLE CHARACTERIZATION}

\begin{tabular}{|l|l|}
\hline \multicolumn{2}{|c|}{ FUEL } \\
\hline Form & He-bonded MOX \\
\hline Composition & $\begin{array}{l}\mathrm{UO2} \text { (natural U)-PuO2; } \\
\text { Pu/(U+Pu) }=0.315 \\
\text { Oxygen-to-metal ratio 1.985 }\end{array}$ \\
\hline Geometry & Annular pellets with flat ends \\
\hline Fissile height & $91.4 \mathrm{~cm}$ \\
\hline $\begin{array}{l}\text { Smear } \\
\text { density }\end{array}$ & $79 \%$ of theoretical density \\
\hline
\end{tabular}

\begin{tabular}{|l|l|}
\hline \multicolumn{2}{|c|}{ CLADDING } \\
\hline Material & SS316 20\% CW \\
\hline OD & $0.584 \mathrm{~cm}$ \\
\hline Thickness & $0.038 \mathrm{~cm}$ \\
\hline
\end{tabular}

PRE-IRRADIATION CHARACTERISTICS

\begin{tabular}{|l|l|}
\hline BU (peak axial) & No pre-irradiation \\
\hline Peak linear power & (not applicable) \\
\hline $\begin{array}{l}\text { Peak internal clad } \\
\text { temp. }\end{array}$ & (not applicable) \\
\hline $\begin{array}{l}\text { Fluence } \\
\text { (spectrum) }\end{array}$ & (not applicable) \\
\hline
\end{tabular}

\section{OTHER FUEL SAMPLE INFORMATION}

Prototypic PFR fuel pins; $159.1 \mathrm{~g} /$ pin fuel mass;

$\sim 1.8 \mathrm{~mm}$ central hole in fuel;

Annular breeder pellets of natural $\mathrm{UO}_{2}(10 \mathrm{~cm}$ upper, 46

$\mathrm{cm}$ lower); $15.4 \mathrm{~cm}^{3}$ bottom plenum at $4.19 \mathrm{~atm}$ (cold)

\begin{tabular}{|l|l|}
\hline \multicolumn{2}{|c|}{ TEST CONDITIONS, MEASUREMENTS, \& ANALYSES } \\
\hline $\begin{array}{l}\text { Coolant } \\
\text { environment }\end{array}$ & Flowing sodium, initially at 30\% of nominal \\
\hline $\begin{array}{l}\text { Transient } \\
\text { conditions }\end{array}$ & $\begin{array}{l}\text { Several seconds at 44 kW/m (average pin at the } \\
\text { midplane) with flow reduction by 50\% in 5 s } \\
\text { inducing boiling and triggering power rise with } \\
0.44 \text { s period for one e-fold, then rise on 0.18 s } \\
\text { period reaching 920 kW/m in 0.35 s, then } \\
\text { scram. }\end{array}$ \\
\hline $\begin{array}{l}\text { Fuel sample } \\
\text { configuration } \\
\text { in the test }\end{array}$ & 7-pin hexagonal bundle; grid spaced pins \\
\hline $\begin{array}{l}\text { Test vehicle \& } \\
\text { experiment } \\
\text { system }\end{array}$ & Mark-IIIC loop, dysprosium shaping collars \\
\hline $\begin{array}{l}\text { Measurements } \\
\text { /diagnostics } \\
\text { during test }\end{array}$ & $\begin{array}{l}\text { Coolant channel flow rate and pressure, } \\
\text { temperatures, fuel motion (hodoscope) }\end{array}$ \\
\hline $\begin{array}{l}\text { Posttest } \\
\text { coolant flow } \\
\text { condition }\end{array}$ & $\begin{array}{l}\text { Upper blockage of once-molten fuel and steel, } \\
\text { complete lower blockage of once-mol ten steel }\end{array}$ \\
\hline $\begin{array}{l}\text { Posttest } \\
\text { condition of } \\
\text { test sample(s) }\end{array}$ & $\begin{array}{l}\text { Gross fuel and cladding disruption (severe } \\
\text { melting and movement), flowtube melt-through }\end{array}$ \\
\hline $\begin{array}{l}\text { Posttest } \\
\text { measurements } \\
\text { \& information }\end{array}$ & $\begin{array}{l}\text { Neutron radiography and tomography, macro } \\
\text { examination, fuel and cladding distribution }\end{array}$ \\
\hline $\begin{array}{l}\text { Posttest } \\
\text { analyses }\end{array}$ & $\begin{array}{l}\text { Pin failure, thermal-hydraulics, post-failure fue } \\
\text { motion, code validation }\end{array}$ \\
\hline
\end{tabular}

\section{KEY SUMMARY INFORMATION ABOUT THE TEST}

LO6 was a fresh-fuel counterpart to LO4, providing a comparison of effects due to fission gas (in irradiated fuel) vs. fuel vapor pressure (in fresh fuel) on fuel dispersal. Lead subassembly conditions were created by causing sodium voiding prior to the planned power burst. (The first part of the power burst was to simulate reactivity increase due to coolant voiding in lead channels in a large fast reactor; the second part of the burst was to simulate reactivity from voiding progression into assemblies of intermediate power.) Prior to cladding failure there was significant fuel expansion but not fuel motion. Cladding failed at fuel height location $x / L=0.72$, followed by severe cladding disruption and dispersive fuel movement. The fresh fuel was less mobile than was the preirradiated fuel in LO4 (and in LO5 and LO7). The final fuel configuration corresponded to a reactivity worth decrease of $\sim 13 \%$ in a large fast reactor. Blockage of once-molten fuel and steel filling two-thirds of flow channel at top of fuel region; 18-cm-long complete lower blockage of once-molten steel at bottom of original fuel region. 


\section{Summary of TREAT Test LO7 \\ Conducted 16 December 1983}

Transient No. 2520

\section{PRE-TEST SAMPLE CHARACTERIZATION}

\begin{tabular}{|l|l|}
\hline \multicolumn{2}{|c|}{ FUEL } \\
\hline Form & He-bonded MOX \\
\hline Composition & $\begin{array}{l}\text { UO2 (natural U)-PuO2; } \\
\text { Pu/(U+Pu) = 0.315 } \\
\text { Oxygen-to-metal ratio 1.985 }\end{array}$ \\
\hline Geometry & Annular pellets with flat ends \\
\hline Fissile height & $91.4 \mathrm{~cm}$ \\
\hline $\begin{array}{l}\text { Smear } \\
\text { density }\end{array}$ & $79 \%$ of theoretical \\
\hline
\end{tabular}

\begin{tabular}{|l|l|}
\hline \multicolumn{2}{|c|}{ CLADDING } \\
\hline Material & SS316 20\% CW \\
\hline OD & $0.584 \mathrm{~cm}$ \\
\hline Thickness & $0.038 \mathrm{~cm}$ \\
\hline
\end{tabular}

PRE-IRRADIATION CHARACTERISTICS

\begin{tabular}{|l|l|}
\hline BU (peak axial) & 4.17 at\% of $\mathrm{HM}$ \\
\hline Peak linear power & $\sim 30 \mathrm{~kW} / \mathrm{m}$ equivalent \\
\hline $\begin{array}{l}\text { Peak internal clad } \\
\text { temperature }\end{array}$ & (not available) \\
\hline $\begin{array}{l}\text { Fluence } \\
\text { (spectrum) }\end{array}$ & $\begin{array}{l}\text { Fluence not available; fast } \\
\text { spectrum }\end{array}$ \\
\hline
\end{tabular}

\section{OTHER FUEL SAMPLE INFORMATION}

Prototypic PFR fuel pins; $159.1 \mathrm{~g} /$ pin fuel mass :

Annular breeder pellets of natural $\mathrm{UO} 2(10 \mathrm{~cm}$ upper, 46 $\mathrm{cm}$ lower); $15.4 \mathrm{~cm} 3$ bottom plenum at $4.19 \mathrm{~atm}$ (cold) ;

As-irradiated low-power structure, $0.09 \mathrm{~cm}$ central hole,

$1.61 \times 10-3$ moles total fission gas release $(29 \%$ of gas generated)

\begin{tabular}{|l|l|}
\hline \multicolumn{2}{|c|}{ TEST CONDITIONS, MEASUREMENTS, \& ANALYSES } \\
\hline $\begin{array}{l}\text { Coolant } \\
\text { environment }\end{array}$ & $\begin{array}{l}\text { Flowing sodium; flow rate held at 43\% of } \\
\text { nominal }\end{array}$ \\
\hline $\begin{array}{l}\text { Transient } \\
\text { conditions }\end{array}$ & $\begin{array}{l}\text { Several seconds at 44 kW/m (average pin at } \\
\text { midplane), then power rise with 0.44 s period for } \\
\text { one e-fold, then on } 50 \text { ms period to } ~ 1610 \\
\text { kW/m in } ~ 0.17 \text { s, then scram. Total energy } \\
\sim 1600 \text { kW/kg in average pin at midplane. }\end{array}$ \\
\hline $\begin{array}{l}\text { Fuel sample } \\
\text { configuration } \\
\text { in the test }\end{array}$ & 7-pin hexagonal bundle; grid-spaced pins \\
\hline $\begin{array}{l}\text { Test vehicle \& } \\
\text { experiment } \\
\text { system }\end{array}$ & Mark-IIICloop; dysprosium shaping collars \\
\hline $\begin{array}{l}\text { Measurements } \\
\text { /diagnostics } \\
\text { during test }\end{array}$ & $\begin{array}{l}\text { Coolant channel flow rate and pressure, } \\
\text { temperatures, fuel motion (hodoscope) }\end{array}$ \\
\hline $\begin{array}{l}\text { Posttest } \\
\text { coolant flow } \\
\text { condition }\end{array}$ & $\begin{array}{l}\text { Total blockage of once-molten fuel and steel } \\
\text { around spacer grids, lower blockage of once- } \\
\text { molten steel }\end{array}$ \\
\hline $\begin{array}{l}\text { Posttest } \\
\text { condition of } \\
\text { test sample(s) }\end{array}$ & $\begin{array}{l}\text { Gross fuel and cladding disruption (severe } \\
\text { melting and movement), flowtube melt-through, } \\
\text { pellet stack collapse }\end{array}$ \\
\hline $\begin{array}{l}\text { Posttest } \\
\text { measurements } \\
\text { \& information }\end{array}$ & $\begin{array}{l}\text { Neutron radiography and tomography, macro } \\
\text { examination, fuel and cladding distribution }\end{array}$ \\
\hline $\begin{array}{l}\text { Posttest } \\
\text { analyses }\end{array}$ & $\begin{array}{l}\text { Pin failure, thermal-hydraulics, post-failure fuet } \\
\text { motion, code validation }\end{array}$ \\
\hline
\end{tabular}

\section{KEY SUMMARY INFORMATION ABOUT THE TEST}

LO7 simulated intermediate subassembly conditions by causing the planned power burst to occur such that pins would fail into single-phase sodium. Prior to cladding failure there was significant fuel expansion but not fuel motion. The faster burst compared to the one in tests LO4-LO6 was to allow investigation of the special case of possible fuel accumulation near the failure site in fast-burst transient-undercooling-driven overpower accidents. Pin failure occurred initially near the midplane when the cladding was still strong, with fuel escaping into a channel that was essentially unvoided at that time and location. The resulting coolant dynamics were much stronger than in LO4 and LO5 and were accompanied by sharp pressure spikes suggestive of molten fuel-coolant interaction. Cladding damage was severe, and dispersive fuel movement corresponded to an overall reactivity worth net loss of approximately $4 \%$. There was a total blockage of once-molten fuel and steel around an upper spacer grid and a nearly-complete blockage of once-molten steel at the bottom of the original fuel region. 


\section{Appendix E: One-page Summaries of M-series Tests}

One-page summaries of each of the M-series tests are included here. These summaries can also be found in TREXR [6]. 


\section{Summary of TREAT Test M1}

Conducted 10 May 1985

Transient No. 2608

\begin{tabular}{l}
\hline \multicolumn{2}{|l|}{ PRE-TEST SAMPLE CHARACTERIZATION } \\
\begin{tabular}{|l|l|}
\hline \multicolumn{2}{|c|}{ FUEL } \\
\hline Form & $\begin{array}{l}\text { Lateral segments of clad fuel cut } \\
\text { from fuel pin }\end{array}$ \\
\hline Composition & U-5Fs \\
\hline Geometry & Two fuel segments, separated \\
\hline Fissile height & $1.27 \mathrm{~cm}$, each segment \\
\hline $\begin{array}{l}\text { Smear } \\
\text { density }\end{array}$ & $\begin{array}{l}\sim 75 \% \text { of theoretical } \\
\text { (17.6 g/cc as-cast) }\end{array}$ \\
\hline
\end{tabular}
\end{tabular}

\begin{tabular}{|l|l|}
\hline \multicolumn{2}{|c|}{ CLADDING } \\
\hline Material & 316 stainless steel, SA \\
\hline OD & $0.442 \mathrm{~cm}$ \\
\hline Thickness & $0.0305 \mathrm{~cm}$ \\
\hline
\end{tabular}

\begin{tabular}{|l|l|}
\hline PRE-IRRADIATION CHARACTERISTICS \\
\hline BU (peak axial) & 3.5 at. \% \\
\hline Peak linear power & (not available) \\
\hline $\begin{array}{l}\text { Peak internal clad } \\
\text { temperature }\end{array}$ & (not available) \\
\hline $\begin{array}{l}\text { Fluence } \\
\text { (spectrum) }\end{array}$ & $\begin{array}{l}\text { Fluence not available; fast } \\
\text { spectrum }\end{array}$ \\
\hline
\end{tabular}

\section{OTHER FUEL SAMPLE INFORMATION}

Sodium was vacuum-distilled out of the samples before

TREAT testing to prevent or limit sodium vapor from

obscuring the visual observation of the fuel.

\begin{tabular}{|c|c|}
\hline \multicolumn{2}{|c|}{ TEST CONDITIONS, MEASUREMENTS, \& ANALYSES } \\
\hline $\begin{array}{l}\text { Coolant } \\
\text { environment }\end{array}$ & No codant; inert gas environment \\
\hline $\begin{array}{l}\text { Transient } \\
\text { conditions }\end{array}$ & $\begin{array}{l}\text { Rapid power rise to a level at which the test fuel } \\
\text { is heated at } 70^{\circ} \mathrm{C} / \mathrm{s} \text {, holding at that level for } 30 \\
\mathrm{~s} \text {, then scram. }\end{array}$ \\
\hline $\begin{array}{l}\text { Fuel sample } \\
\text { configuration } \\
\text { in the test }\end{array}$ & $\begin{array}{l}\text { One pin segment open to } 0.255 \mathrm{MPa} \\
\text { containment; the other pin segment encased in } \\
\text { quartz tube at } 2.67 \mathrm{MPa} \text { pressure }\end{array}$ \\
\hline $\begin{array}{l}\text { Test vehicle \& } \\
\text { experiment } \\
\text { system }\end{array}$ & F-series type of dry capsule \\
\hline $\begin{array}{l}\text { Measurements } \\
\text { /diagnostics } \\
\text { during test }\end{array}$ & $\begin{array}{l}\text { High-speed photography with laser illumination; } \\
\text { thermocouples spot-welded to each sample; } \\
\text { pressure transducers; fuel motion by hodoscope }\end{array}$ \\
\hline $\begin{array}{l}\text { Posttest } \\
\text { coolant flow } \\
\text { condition }\end{array}$ & Not applicable \\
\hline $\begin{array}{l}\text { Posttest } \\
\text { condition of } \\
\text { test sample(s) }\end{array}$ & $\begin{array}{l}\text { The segment in high pressure became slighty } \\
\text { bent. The segment in low pressure lost a piece } \\
\text { of fuel and also bent. }\end{array}$ \\
\hline $\begin{array}{l}\text { Posttest } \\
\text { measurements } \\
\text { \& information }\end{array}$ & Neutron radiography \\
\hline $\begin{array}{l}\text { Posttest } \\
\text { analyses }\end{array}$ & Not available \\
\hline
\end{tabular}

\section{KEY SUMMARY INFORMATION ABOUT THE TEST}

The purpose of test M1 was to collect preliminary information regarding the transient-induced extrusion behavior of metallic fuels within cladding. One pin segment was held at low pressure to simulate pin failure conditions, while the other was encased in quartz and held at high plenum pressure simulating the plenum pressure in intact fuel pins. During the test, a chunk fell off the low-pressure segment, followed by a plume of smoke that obscured the remainder of the experiment from the camera's view. The smoke was apparently sodium vapor and other volatiles that had been trapped within the fuel. The fuel in both pins remained intact, and little fuel extrusion or swelling occurred. 


\section{Summary of TREAT Test M2}

Conducted 21 March 1985

Transient No. 2596

\begin{tabular}{|c|c|}
\hline \multicolumn{2}{|c|}{ PRE-TEST SAMPLE CHARACTERIZATION } \\
\hline \multicolumn{2}{|r|}{ FUEL } \\
\hline Form & Sodium-bonded metallic fuel \\
\hline Composition & U-5Fs \\
\hline Geometry & Cast pin \\
\hline Fissile height & $34.3 \mathrm{~cm}$, as fabricated \\
\hline $\begin{array}{l}\text { Smear } \\
\text { density }\end{array}$ & $\begin{array}{l}\sim 75 \% \text { of theoretical } \\
\text { (17.6 g/cc as-cast) }\end{array}$ \\
\hline
\end{tabular}

\begin{tabular}{|l|l|}
\hline \multicolumn{2}{|c|}{ CLADDING } \\
\hline Material & 316 stainless steel, SA \\
\hline OD & $0.442 \mathrm{~cm}$ \\
\hline Thickness & $0.0305 \mathrm{~cm}$ \\
\hline
\end{tabular}

\begin{tabular}{|l|l|}
\hline \multicolumn{2}{|l|}{ PRE-IRRADIATION CHARACTERISTICS } \\
\hline BU (peak axial) & Various; see below \\
\hline Peak linear power & $39.4 \mathrm{~kW} / \mathrm{m}$ \\
\hline $\begin{array}{l}\text { Peak internal clad } \\
\text { temperature }\end{array}$ & (not available) \\
\hline $\begin{array}{l}\text { Fluence } \\
\text { (spectrum) }\end{array}$ & $\begin{array}{l}\text { Fluence not available; } \\
\text { fast spectrum }\end{array}$ \\
\hline
\end{tabular}

\section{OTHER FUEL SAMPLE INFORMATION}

Three pins: peak axial burnups of 0.3 at. $\%, 4.4$ at. \%, and 7.9 at. \%. Each pin was wire-wrapped, with 0.124 $\mathrm{cm}$ diameter wire on a $15.2-\mathrm{cm}$ pitch.

\begin{tabular}{|l|l|}
\hline \multicolumn{2}{|c|}{ TEST CONDITIONS, MEASUREMENTS, \& ANALYSES } \\
\hline $\begin{array}{l}\text { Coolant } \\
\text { environment }\end{array}$ & Flowing sodium; same flow rate for each pin \\
\hline $\begin{array}{l}\text { Transient } \\
\text { conditions }\end{array}$ & $\begin{array}{l}\text { Power rose to nominal power (39 kW/m), held } \\
\text { for } 1.5 \mathrm{~s} \text {, then increased exponentially on an 8- } \\
\text { second period, reaching peak power of about 4 } \\
\text { times nominal. }\end{array}$ \\
\hline $\begin{array}{l}\text { Fuel sample } \\
\text { configuration } \\
\text { in the test }\end{array}$ & $\begin{array}{l}\text { 3 pins, each within its own separate thin-walled } \\
\text { stainless steel flow tube, with a He-Ar gas } \\
\text { mixture around the flow tubes }\end{array}$ \\
\hline $\begin{array}{l}\text { Test vehicle \& } \\
\text { experiment } \\
\text { system }\end{array}$ & Mark-IIIC sodium loop \\
\hline $\begin{array}{l}\text { Measurements } \\
\text { /diagnostics } \\
\text { during test }\end{array}$ & $\begin{array}{l}\text { Coolant pressure, temperature, and flow rate; } \\
\text { flowtube outer surface temperature; fuel motion } \\
\text { monitoring by hodoscope }\end{array}$ \\
\hline $\begin{array}{l}\text { Posttest } \\
\text { coolant flow } \\
\text { condition }\end{array}$ & $\begin{array}{l}\text { Coolant channel pressurization upon cladding } \\
\text { breach caused sharp flow anomaly, including } \\
\text { sharp decrease in inlet flow rate. Final } \\
\text { posttransient flow rate was about 75\% of the } \\
\text { initial flow rate. }\end{array}$ \\
\hline $\begin{array}{l}\text { Posttest } \\
\text { condition of } \\
\text { test sample(s) }\end{array}$ & $\begin{array}{l}\text { f.3\% burnup pin remained intact; other two pins } \\
\text { failed with substantial fuel loss from cladding. } \\
\text { each failed pin. }\end{array}$ \\
\hline $\begin{array}{l}\text { Posttest } \\
\text { measurements } \\
\text { \& information }\end{array}$ & $\begin{array}{l}\text { Neutron radiograph; detailed posttest } \\
\text { metallurgical examinations }\end{array}$ \\
\hline analyses & $\begin{array}{l}\text { Cadding failure analysis; pre- and post-failure } \\
\text { fuel motion analysis; thermal-hydraulic analysis }\end{array}$ \\
\hline
\end{tabular}

\footnotetext{
KEY SUMMARY INFORMATION ABOUT THE TEST

Test M2 investigated the effects of fuel motion before and after cladding failure during an overpower transient with power rise rate fast enough to cause cladding failure primarily by rapid cladding penetration by formation of low-melting-point fuelcladding phases and assisted by plenum pressure. Axial power distribution was 1.10 peak/average. Transient pre-failure axial fuel growth of the low, medium, and high burnup fuel columns was measured to be $16 \%, 15 \%$, and $3 \%$, respectively. Power was immediately shut down after the first cladding failure, at approximately four times nominal power. The failures occurred at the top of the fuel column in the two higher-burnup pins, after which the fuel (or fuel-cladding melt) was rapidly ejected through the breach and moved upward in the flow channel, leaving empty space in the bottom quarter of each pin's original fuel region. This rapid dispersal of fuel can be explained as having been due to sodium vaporization rather than fission gas pressure. Approximately $50 \%$ of the fuel's volume melted before cladding failure occurred. Coolant pressure pulses peaked at about $1.7 \mathrm{MPa}$.
} 


\title{
Summary of TREAT Test M3
}

\author{
Conducted 11 April 1985
}

Transient No. 2599

\section{PRE-TEST SAMPLE CHARACTERIZATION}

\begin{tabular}{|l|l|}
\hline \multicolumn{2}{|c|}{ FUEL } \\
\hline Form & Sodium-bonded metallic fuel \\
\hline Composition & U-5Fs \\
\hline Geometry & Cast pin \\
\hline Fissile height & $34.3 \mathrm{~cm}$, as fabricated \\
\hline $\begin{array}{l}\text { Smear } \\
\text { density }\end{array}$ & $\begin{array}{l}\sim 75 \% \text { of theoretical } \\
(17.6 \mathrm{~g} / \mathrm{cc} \text { as-cast })\end{array}$ \\
\hline
\end{tabular}

\begin{tabular}{|l|l|}
\hline \multicolumn{2}{|c|}{ CLADDING } \\
\hline Material & 316 stainless steel, SA \\
\hline OD & $0.442 \mathrm{~cm}$ \\
\hline Thickness & $0.0305 \mathrm{~cm}$ \\
\hline
\end{tabular}

\begin{tabular}{|l|l|}
\hline \multicolumn{2}{|l|}{ PRE-IRRADIATION CHARACTERISTICS } \\
\hline BU (peak axial) & Various; see below \\
\hline Peak linear power & $39.4 \mathrm{~kW} / \mathrm{m}$ \\
\hline $\begin{array}{l}\text { Peak internal clad } \\
\text { temperature }\end{array}$ & (not available) \\
\hline $\begin{array}{l}\text { Fluence } \\
\text { (spectrum) }\end{array}$ & $\begin{array}{l}\text { Fluence not available; fast } \\
\text { spectrum }\end{array}$ \\
\hline
\end{tabular}

\section{OTHER FUEL SAMPLE INFORMATION}

Three pins: peak axial burnups of 0.3 at. $\%, 4.4$ at. \%, and 7.9 at. \%. Each pin was wire-wrapped, with 0.124 $\mathrm{cm}$ diameter wire on a $15.2-\mathrm{cm}$ pitch.

\begin{tabular}{|l|l|}
\hline \multicolumn{2}{|c|}{ TEST CONDITIONS, MEASUREMENTS, \& ANALYSES } \\
\hline $\begin{array}{l}\text { Coolant } \\
\text { environment }\end{array}$ & Flowing sodium; same flow rate for each pin \\
\hline $\begin{array}{l}\text { Transient } \\
\text { conditions }\end{array}$ & $\begin{array}{l}\text { Power rose to nominal power (39 kW/m), held } \\
\text { for } 1.5 \mathrm{~s} \text {, then increased exponentially on an 8- } \\
\text { second period, reaching peak power of about } 3.8 \\
\text { times nominal. }\end{array}$ \\
\hline $\begin{array}{l}\text { Fuel sample } \\
\text { configuration } \\
\text { in the test }\end{array}$ & $\begin{array}{l}\text { 3 pins, each within its own separate thin-walled } \\
\text { stainless steel flow tube, with a He-Ar gas } \\
\text { mixture around the flow tubes }\end{array}$ \\
\hline $\begin{array}{l}\text { Test vehicle \& } \\
\text { experiment } \\
\text { system }\end{array}$ & Mark-IIIC sodium loop \\
\hline $\begin{array}{l}\text { Measurements } \\
\text { /diagnostics } \\
\text { during test }\end{array}$ & $\begin{array}{l}\text { Coolant pressure, temperature, and flow rate; } \\
\text { flowtube outer surface temperature; fuel motion } \\
\text { monitoring by hodoscope }\end{array}$ \\
\hline $\begin{array}{l}\text { Posttest } \\
\text { coolant flow } \\
\text { condition }\end{array}$ & No significant changes \\
\hline $\begin{array}{l}\text { Posttest } \\
\text { condition of } \\
\text { test sample(s) }\end{array}$ & $\begin{array}{l}\text { All pins intact. Significant axial fuel elongation } \\
\text { occurred in the lowest burnup pin. }\end{array}$ \\
\hline $\begin{array}{l}\text { Posttest } \\
\text { measurements } \\
\text { \& information }\end{array}$ & $\begin{array}{l}\text { Neutron radiograph; detailed metallurgical } \\
\text { examinations }\end{array}$ \\
\hline $\begin{array}{l}\text { Posttest } \\
\text { analyses }\end{array}$ & $\begin{array}{l}\text { Cladding failure predictions, pre-fail ure fuel } \\
\text { motion analysis, thermal-hydraulic analysis }\end{array}$ \\
\hline
\end{tabular}

\footnotetext{
KEY SUMMARY INFORMATION ABOUT THE TEST

Test M3 (like Test M2) investigated the effects of fuel motion during an overpower transient with power rise rate fast enough to cause cladding failure primarily by rapid cladding penetration by formation of low-melting-point fuel-cladding phases and assisted by plenum pressure. However, adjustments were made to the coolant flow rates in each channel to cause the lowestburnup pin to reach failure (or incipient failure) at the time that the higher burnup pins reached incipient failure. Axial power distribution was 1.10 peak/average. The transient was terminated after the power reached 3.8 times nominal. None of the pins failed during the test. Fuel expanded axially by $18 \%$ in the low burnup pin and by $4 \%$ in the medium and high burnup pins.
} 


\title{
Summary of TREAT Test M4
}

\author{
Conducted 13 January 1986
}

Transient No. 2683

\begin{tabular}{|c|c|}
\hline \multicolumn{2}{|c|}{ PRE-TEST SAMPLE CHARACTERIZATION } \\
\hline \multicolumn{2}{|r|}{ FUEL } \\
\hline Form & Sodium-bonded metallic fuel \\
\hline Composition & U-5Fs \\
\hline Geometry & Cast pin \\
\hline Fissile height & $34.3 \mathrm{~cm}$, as fabricated \\
\hline $\begin{array}{l}\text { Smear } \\
\text { density }\end{array}$ & $\begin{array}{l}\sim 75 \% \text { of theoretical } \\
\text { (17.6 g/cc as-cast) }\end{array}$ \\
\hline
\end{tabular}

\begin{tabular}{|l|l|}
\hline \multicolumn{2}{|c|}{ CLADDING } \\
\hline Material & 316 stainless steel, SA \\
\hline OD & $0.442 \mathrm{~cm}$ \\
\hline Thickness & $0.0305 \mathrm{~cm}$ \\
\hline
\end{tabular}

\begin{tabular}{|l|l|}
\hline \multicolumn{2}{|l|}{ PRE-IRRADIATION CHARACTERISTICS } \\
\hline BU (peak axial) & Various; see below \\
\hline Peak linear power & $39.4 \mathrm{~kW} / \mathrm{m}$ \\
\hline $\begin{array}{l}\text { Peak internal clad } \\
\text { temperature }\end{array}$ & (not available) \\
\hline $\begin{array}{l}\text { Fluence } \\
\text { (spectrum) }\end{array}$ & $\begin{array}{l}\text { Fuence not available; fast } \\
\text { spectrum }\end{array}$ \\
\hline
\end{tabular}

\section{OTHER FUEL SAMPLE INFORMATION}

Two pre-irradiated pins (burnups 4.4 at\% and 2.4 at\%)

and one fresh (unirradiated) pin were tested.

\begin{tabular}{|l|l|}
\hline \multicolumn{2}{|c|}{ TEST CONDITIONS, MEASUREMENTS, \& ANALYSES } \\
\hline $\begin{array}{l}\text { Coolant } \\
\text { environment }\end{array}$ & Flowing sodium \\
\hline $\begin{array}{l}\text { Transient } \\
\text { conditions }\end{array}$ & $\begin{array}{l}\text { Power rose to nominal power (39 held for 1.5 s, } \\
\text { then increased exponentially on an 8-second } \\
\text { period, reaching peak power of about } 4 \text { times } \\
\text { nominal. }\end{array}$ \\
\hline $\begin{array}{l}\text { Fuel sample } \\
\text { configuration } \\
\text { in the test }\end{array}$ & $\begin{array}{l}\text { 3 pins, each within its own separate thin-walled } \\
\text { stainless steel flow tube, with a He-Ar gas } \\
\text { mixture around the flow tubes }\end{array}$ \\
\hline $\begin{array}{l}\text { Test vehicle \& } \\
\text { experiment } \\
\text { system }\end{array}$ & Mark-IIIC sodium loop \\
\hline $\begin{array}{l}\text { Measurements } \\
\text { /diagnostics } \\
\text { during test }\end{array}$ & $\begin{array}{l}\text { Coolant pressure, temperature, and flow rate; } \\
\text { flowtube outer surface temperature; fuel motion } \\
\text { monitoring by hodoscope }\end{array}$ \\
\hline $\begin{array}{l}\text { Posttest } \\
\text { coolant flow } \\
\text { condition }\end{array}$ & $\begin{array}{l}\text { Coolant channel pressurization upon cladding } \\
\text { breach caused sharp flow anomaly, including } \\
\text { sharp decrease in inlet flow rate. Final } \\
\text { posttransient flow rate was approximately } 90 \% \\
\text { of the initial flow rate. }\end{array}$ \\
\hline $\begin{array}{l}\text { Posttest } \\
\text { condition of } \\
\text { test sample(s) }\end{array}$ & $\begin{array}{l}\text { The 2.4 at\% burnup pin failed, with a small } \\
\text { breach near the top of the fuel column. The } \\
\text { bottom quarter of the fuel column remained in } \\
\text { place. About } 1 / 3 \text { of the rest of the fuel was } \\
\text { expelled from the cladding. The other two pins } \\
\text { remained intact. }\end{array}$ \\
\hline $\begin{array}{l}\text { Posttest } \\
\text { measurements } \\
\text { anformation }\end{array}$ & $\begin{array}{l}\text { Neutron radiograph; detailed metallurgical } \\
\text { examinations. }\end{array}$ \\
\hline $\begin{array}{l}\text { Cadding failure analysis, pre- and post-failure } \\
\text { fuel motion analysis, thermal-hydraulic analysis }\end{array}$ \\
\hline
\end{tabular}

\section{KEY SUMMARY INFORMATION ABOUT THE TEST}

Test M4 focused on three issues pertaining to pre-failure fuel elongation: (1) the role of trapped sodium versus fission gas in very-low-burnup fuel, (2) the amount that would occur in fuel of burnup between the very-low and medium burnup fuels tested in $M 2$ and $M 3$, and (3) additional information to resolve the discrepancy measured in the 4.4 at\% burnup fuel tested in M2 and $M 3$. Compared to $M 2$ and $M 3$, a higher flow rate and lower sodium inlet temperature caused the fuel and cladding temperatures at the time of failure to be more prototypic of fast reactor conditions. The fuel melt radius was therefore larger at failure. The transient was quickly terminated when the 2.4 at\% burnup pin failed, at about 4.1 times nominal power for that pin. Fuel (or fuel-cladding melt) was ejected from that pin through a small breach near the top of the fuel column and swept upward. The 4.4 at\% burnup fuel elongated about the same amount as in M3. The fresh fuel elongated about $3 \%$, due to local swelling at the top, then slumped to about $90 \%$ of the initial height after the transient was terminated. Data from M2, M3, and M4 confirm that significant fuel extrusion does not occur until melting, and fission gas serves as the primary cause of extrusion, although sodium boiling also has an effect at low plenum pressures. 


\section{Summary of TREAT Test M5}

Conducted 15 August 1986

Transient No. 2714

PRE-TEST SAMPLE CHARACTERIZATION
\begin{tabular}{|l|l|}
\hline \multicolumn{2}{|c|}{ FUEL } \\
\hline Form & Sodium-bonded metallic fuel \\
\hline Composition & U-19Pu-10Zr \\
\hline Geometry & Cast fuel slug \\
\hline Fissile height & $34.3 \mathrm{~cm}$ (as-fabricated) \\
\hline $\begin{array}{l}\text { Smear } \\
\text { density }\end{array}$ & $72.5 \%$ of theoretical \\
\hline
\end{tabular}

\begin{tabular}{|l|l|}
\hline \multicolumn{2}{|c|}{ CLADDING } \\
\hline Material & D9 Steel \\
\hline OD & $0.584 \mathrm{~cm}$ \\
\hline Thickness & $0.038 \mathrm{~cm}$ \\
\hline
\end{tabular}

\begin{tabular}{|l|l|}
\hline \multicolumn{2}{|l|}{ PRE-IRRADIATION CHARACTERISTICS } \\
\hline BU (peak axial) & $\begin{array}{l}0.8 \text { at\% in one pin; } \\
1.9 \text { at\% in the other }\end{array}$ \\
\hline Peak linear power & 36 to $40 \mathrm{~kW} / \mathrm{m}$ \\
\hline $\begin{array}{l}\text { Peak internal clad } \\
\text { temperature }\end{array}$ & (not available) \\
\hline $\begin{array}{l}\text { Fluence } \\
\text { (spectrum) }\end{array}$ & $\begin{array}{l}\text { Fluence not available; fast } \\
\text { spectrum }\end{array}$ \\
\hline
\end{tabular}

\section{OTHER FUEL SAMPLE INFORMATION}

$5.0 \mathrm{cc}$ as-fabricated gas plenum volume.

2 to $3 \%$ as-irradiated axial swelling.

Wire wraps were removed prior to the test.

\begin{tabular}{|c|c|}
\hline \multicolumn{2}{|c|}{ TEST CONDITIONS, MEASUREMENTS, \& ANALYSES } \\
\hline $\begin{array}{l}\text { Coolant } \\
\text { environment }\end{array}$ & Flowing sodium; $0.4 \mathrm{MPa}$ system pressure \\
\hline $\begin{array}{l}\text { Transient } \\
\text { conditions }\end{array}$ & $\begin{array}{l}\text { Power increased quickly to nominal power, then } \\
\text { increased exponentially on an 8-s period. }\end{array}$ \\
\hline $\begin{array}{l}\text { Fuel sample } \\
\text { configuration } \\
\text { in the test }\end{array}$ & $\begin{array}{l}\text { Two pins, each located in its own } 0.038 \mathrm{~cm} \text {-thick } \\
\text { stainless steel flowtube with inert gas between } \\
\text { the flowtubes. Dimples in flowtubes centered the } \\
\text { pins in the tubes. }\end{array}$ \\
\hline $\begin{array}{l}\text { Test vehicle \& } \\
\text { experiment } \\
\text { system }\end{array}$ & Mark-IIIC sodium loop \\
\hline $\begin{array}{l}\text { Measurements } \\
\text { /diagnostics } \\
\text { during test }\end{array}$ & $\begin{array}{l}\text { Coolant inlet and outlet pressures, temperatures, } \\
\text { and total flow rates, plus flow rate in each } \\
\text { flowtube; temperatures on the outer surfaces of } \\
\text { each flow tube; fuel motion by hodoscope }\end{array}$ \\
\hline $\begin{array}{l}\text { Posttest } \\
\text { coolant flow } \\
\text { condition }\end{array}$ & Coolant boiled at end of test \\
\hline $\begin{array}{l}\text { Posttest } \\
\text { condition of } \\
\text { test sample(s) }\end{array}$ & Both fuel pins remained intact \\
\hline $\begin{array}{l}\text { Posttest } \\
\text { measurements } \\
\text { \& information }\end{array}$ & $\begin{array}{l}\text { Neutron radiograph; detailed metallurgical } \\
\text { examinations }\end{array}$ \\
\hline $\begin{array}{l}\text { Posttest } \\
\text { analyses }\end{array}$ & $\begin{array}{l}\text { Cladding failure, pre- and post-failure fuel } \\
\text { motion, thermal-hydraulics }\end{array}$ \\
\hline
\end{tabular}

\section{KEY SUMMARY INFORMATION ABOUT THE TEST}

Test M5 investigated the pre-failure axial expansion of IFR zirconium-based fuel, which was not available in time to be used in tests M2, M3, or M4. The test was intended to heat the fuel pins not quite to failure (so as to be able to reuse the loop for a subsequent test). After an initial transient that reached 3.3 times nominal power, the hodoscope data showed no fuel movement occurred. The same power transient was then rerun, but the sodium flow rate was reduced by $22 \%$. Both pins remained intact. The higher flowrate in the second run caused the fuel temperatures and melt radius to be less than prototypic. Peak power-to-flow ratio was 4.4 times nominal in the $0.8 \%$ burnup pin and 4.2 times nominal in the $1.9 \%$ burnup pin. Transient-induced fuel elongation was 2 to $3 \%$. There was evidence of eutectic fuel-cladding interaction in which a multiphase alloy was formed, a behavior not previously observed in the U-Fs fuel of tests M2 - M4. 


\section{Summary of TREAT Test M6}

Conducted 6 February 1987

Transient No. 2734

PRE-TEST SAMPLE CHARACTERIZATION
\begin{tabular}{|l|l|}
\hline \multicolumn{2}{|c|}{ FUEL } \\
\hline Form & Sodium-bonded metallic fuel \\
\hline Composition & U-19Pu-10Zr \\
\hline Geometry & Cast fuel slug \\
\hline Fissile height & $34.3 \mathrm{~cm}$ (as-fabricated) \\
\hline $\begin{array}{l}\text { Smear } \\
\text { density }\end{array}$ & $72.5 \%$ of theoretical \\
$11.2 \mathrm{~g} / \mathrm{cc}$ \\
\hline
\end{tabular}

\begin{tabular}{|l|l|}
\hline \multicolumn{2}{|c|}{ CLADDING } \\
\hline Material & D9 Steel \\
\hline OD & $0.584 \mathrm{~cm}$ \\
\hline Thickness & $0.038 \mathrm{~cm}$ \\
\hline
\end{tabular}

\begin{tabular}{|l|l|}
\hline PRE-IRRADIATION CHARACTERISTICS \\
\hline BU (peak axial) & $\begin{array}{l}1.9 \text { at\% in one pin; } \\
5.3 \text { at\% in the other }\end{array}$ \\
\hline Peak linear power & 36 to $40 \mathrm{~kW} / \mathrm{m}$ \\
\hline $\begin{array}{l}\text { Peak internal clad } \\
\text { temperature }\end{array}$ & (not available) \\
\hline $\begin{array}{l}\text { Fluence } \\
\text { (spectrum) }\end{array}$ & $\begin{array}{l}\text { Fluence not available; fast } \\
\text { spectrum }\end{array}$ \\
\hline
\end{tabular}

\section{OTHER FUEL SAMPLE INFORMATION}

$5.0 \mathrm{cc}$ as-fabricated gas plenum volume.

2 to $3 \%$ as-irradiated axial swelling.

Wire wraps were removed prior to the test.

\begin{tabular}{|l|l|}
\hline \multicolumn{2}{|c|}{ TEST CONDITIONS, MEASUREMENTS, \& ANALYSES } \\
\hline $\begin{array}{l}\text { Coolant } \\
\text { environment }\end{array}$ & Flowing sodium; 0.4 MPa system pressure. \\
\hline $\begin{array}{l}\text { Transient } \\
\text { conditions }\end{array}$ & $\begin{array}{l}\text { Power was raised first to approximately nominal } \\
\text { power, the increased exponentially on an 8-s } \\
\text { period until cladding failure was reached, then } \\
\text { sharply reduced. }\end{array}$ \\
\hline $\begin{array}{l}\text { Fuel sample } \\
\text { configuration } \\
\text { in the test }\end{array}$ & $\begin{array}{l}\text { Two pins, each located in its own 0.038 cm-thick } \\
\text { stainless steel flowtube with inert gas between } \\
\text { the flowtubes. }\end{array}$ \\
\hline $\begin{array}{l}\text { Test vehicle \& } \\
\text { experiment } \\
\text { system }\end{array}$ & Mark-IIIC sodium loop \\
\hline $\begin{array}{l}\text { Measurements } \\
\text { diagnostics } \\
\text { during test }\end{array}$ & $\begin{array}{l}\text { Coolant inlet and outlet pressures, temperatures, } \\
\text { and total flow rates, plus flow rate in each } \\
\text { flowtube; temperatures on the outer surfaces of } \\
\text { each flow tube; fuel motion by hodoscope }\end{array}$ \\
\hline $\begin{array}{l}\text { Posttest } \\
\text { coolant flow } \\
\text { condition }\end{array}$ & $\begin{array}{l}\text { Temporary reversal of flow in the flowtube of the } \\
5.3 \text { at\% burnup pin. }\end{array}$ \\
\hline $\begin{array}{l}\text { Posttest } \\
\text { condition of } \\
\text { test sample(s) }\end{array}$ & $\begin{array}{l}\text { 5.3 at.\% pin failed, releasing fuel into codant } \\
\text { flowtube. }\end{array}$ \\
\hline $\begin{array}{l}\text { Posttest } \\
\text { measurements } \\
\text { \& information }\end{array}$ & $\begin{array}{l}\text { Neutron radiograph; detailed metallurgical } \\
\text { examinations }\end{array}$ \\
\hline $\begin{array}{l}\text { Posttest } \\
\text { analyses }\end{array}$ & $\begin{array}{l}\text { Cadding failure analysis, pre- and post-failure } \\
\text { fuel motion analyses, thermal-hydraulic analysis }\end{array}$ \\
\hline
\end{tabular}

\section{KEY SUMMARY INFORMATION ABOUT THE TEST}

Test M6 was identical to M5 but used fuel samples with higher levels of pre-irradiation. The 5.3 at.\% pin failed at 4.1 times nominal power, at the top of the fuel column; the 1.9 at\% burnup pin reached 4.2 times nominal power and remained intact. The failure of the 5.3 at\% pin was accompanied by the upward expulsion into the coolant channel of two thirds of the fuel (or fuel-cladding melt) through the localized failure, yet more than half of the original flow rate was maintained in that flowtube. Pre-failure elongation was $4 \%$ in both pins, supporting the observation in M5 (relative to M2, M3, and M4) that IFR fuels exhibit less elongation than U-Fs fuel, probably because the higher operating temperature of ternary fuel leads to lower concentrations of retained fission gas. 


\section{Summary of TREAT Test M7}

Conducted 15 October 1987

Transient No. 2775

PRE-TEST SAMPLE CHARACTERIZATION
\begin{tabular}{|l|l|}
\hline \multicolumn{2}{|c|}{ FUEL } \\
\hline Form & Sodium-bonded metallic fuel \\
\hline Composition & $\begin{array}{l}\text { U-19Pu-10Zr in one pin; } \\
\text { U-10Zr in the other }\end{array}$ \\
\hline Geometry & Cast fuel slug \\
\hline Fissile height & $34.3 \mathrm{~cm}$ (as-fabricated) \\
\hline $\begin{array}{l}\text { Smear } \\
\text { density }\end{array}$ & $\begin{array}{l}72.5 \% \text { of theoretical } \\
11.2 \mathrm{~g} / \mathrm{cc}\end{array}$ \\
\hline
\end{tabular}

\begin{tabular}{|l|l|}
\hline \multicolumn{2}{|c|}{ CLADDING } \\
\hline Material & $\begin{array}{l}\text { D9 steel for the U-Pu-Zr fuel; } \\
\text { HT9 steel for the U-Zr fuel }\end{array}$ \\
\hline OD & $0.584 \mathrm{~cm}$ \\
\hline Thickness & $0.038 \mathrm{~cm}$ \\
\hline
\end{tabular}

\begin{tabular}{|l|l|}
\hline \multicolumn{2}{|l|}{ PRE-IRRADIATION CHARACTERISTICS } \\
\hline BU (peak axial) & $\begin{array}{l}9.8 \text { at\% in U-Pu-Zr pin; } \\
2.9 \text { at\% in U-Zr pin }\end{array}$ \\
\hline Peak linear power & $38 \mathrm{~kW} / \mathrm{m}$ \\
\hline $\begin{array}{l}\text { Peak internal clad } \\
\text { temperature }\end{array}$ & (not available) \\
\hline $\begin{array}{l}\text { Fluence } \\
\text { (spectrum) }\end{array}$ & $\begin{array}{l}\text { Fluence not available; fast } \\
\text { spectrum }\end{array}$ \\
\hline
\end{tabular}

\section{OTHER FUEL SAMPLE INFORMATION}

\section{$5.0 \mathrm{cc}$ as-fabricated gas plenum volume.}

2 to $3 \%$ as-irrad. axial fuel swelling (UPuZr pin),

8 to $9 \%$ as-irrad. axial fuel swelling (UZr pin),

1.09 maxlavg axial burnup distribution,

Wire wraps were removed prior to the test.

\begin{tabular}{|l|l|}
\hline \multicolumn{2}{|c|}{ TEST CONDITIONS, MEASUREMENTS, \& ANALYSES } \\
\hline $\begin{array}{l}\text { Coolant } \\
\text { environment }\end{array}$ & Flowing sodium; 0.4 MPa system pressure \\
\hline $\begin{array}{l}\text { Transient } \\
\text { conditions }\end{array}$ & $\begin{array}{l}\text { Power increased exponentially for 8 s until } \\
\text { cladding failure was reached, then sharply } \\
\text { reduced. }\end{array}$ \\
\hline $\begin{array}{l}\text { Fuel sample } \\
\text { configuration } \\
\text { in the test }\end{array}$ & $\begin{array}{l}\text { Two pins, each located in its own 0.038 cm-thick } \\
\text { stainless steel flowtube with inert gas between } \\
\text { the flowtubes }\end{array}$ \\
\hline $\begin{array}{l}\text { Test vehicle \& } \\
\text { experiment } \\
\text { system }\end{array}$ & Mark-IIIC sodium loop \\
\hline $\begin{array}{l}\text { Measurements } \\
\text { /diagnostics } \\
\text { during test }\end{array}$ & $\begin{array}{l}\text { Coolant inlet and outlet pressures, temperatures, } \\
\text { and total flow rates, plus flow rate in each } \\
\text { flowtube; temperatures on the outer surfaces of } \\
\text { each flow tube; fuel motion by hodoscope }\end{array}$ \\
\hline $\begin{array}{l}\text { Posttest } \\
\text { coolant flow } \\
\text { condition }\end{array}$ & $\begin{array}{l}\text { Temporary reversal of flow, followed by } \\
\text { restoration of 0.7 times prefailure flow rate in } \\
\text { flowtube of failed pin. }\end{array}$ \\
\hline $\begin{array}{l}\text { Posttest } \\
\text { condition of } \\
\text { test sample(s) }\end{array}$ & $\begin{array}{l}\text { U-19Pu-10Zr pin failed, releasing fuel into the } \\
\text { coolant flowtube through a small breach near the } \\
\text { top of the fuel column. The U-10Zr pin did not } \\
\text { fail. }\end{array}$ \\
\hline $\begin{array}{l}\text { Posttest } \\
\text { measurements } \\
\text { \& information }\end{array}$ & $\begin{array}{l}\text { Neutron radiography, isotopic gamma scanning, } \\
\text { laser profilometry, plenum gas analysis, macro- } \\
\text { and microphotography }\end{array}$ \\
\hline $\begin{array}{l}\text { Posttest } \\
\text { analyses }\end{array}$ & $\begin{array}{l}\text { Cadding failure, pre- and post-failure fuel } \\
\text { motion, thermal-hydraulics }\end{array}$ \\
\hline
\end{tabular}

\section{KEY SUMMARY INFORMATION ABOUT THE TEST}

In test M7, the binary (UZr) fuel sample survived a power of 4.8 times nominal, with up to one-third of the cladding thickness being lost locally near the top of the fuel column due to transient-induced fuel-cladding interaction. The ternary (UPuZr) fuel pin failed at 4.0 times nominal, rapidly ejecting half of its fuel (or molten mixture of fuel and cladding) through a breach in the cladding at the top of the fuel column into the coolant flowtube, where the melt continued moving upwards. As in tests M5 and M6, at peak power about half of the fuel inventory was molten. 


\section{Appendix F: One-page Summaries of TS-series Tests}

One-page summaries of each of the TS-series tests are included here. These summaries can also be found in TREXR [6]. 


\section{Summary of TREAT Test TS-1

\author{
Conducted 8 August 1983
}

Transient No. 2474

\section{PRE-TEST SAMPLE CHARACTERIZATION}

\begin{tabular}{|l|l|}
\hline \multicolumn{2}{|c|}{ FUEL } \\
\hline Form & He-bonded MOX \\
\hline Composition & $\mathrm{UO}_{2}$ (Natural) $-22.4 \mathrm{w}_{0} \mathrm{PuO}_{2}$ \\
\hline Geometry & Solid, dished pellet \\
\hline Fissile height & $91.4 \mathrm{~cm}$ \\
\hline $\begin{array}{l}\text { Smear } \\
\text { density }\end{array}$ & $85.6 \%$ of theoretical \\
\hline
\end{tabular}

\begin{tabular}{|l|l|}
\hline \multicolumn{2}{|c|}{ CLADDING } \\
\hline Material & $20 \%$ cold-worked type 316 SS \\
\hline OD & $0.584 \mathrm{~cm}$ \\
\hline Thickness & $0.038 \mathrm{~cm}$ \\
\hline
\end{tabular}

\begin{tabular}{|l|l|}
\hline \multicolumn{2}{|l|}{ PRE-IRRADIATION CHARACTERISTICS } \\
\hline BU (peak axial) & $\sim 2 \mathrm{MWd} / \mathrm{kg}(0.2 \%$ of $\mathrm{HM})$ \\
\hline Peak linear power & $41 \mathrm{~kW} / \mathrm{m}$ \\
\hline $\begin{array}{l}\text { Peak internal clad } \\
\text { temperature }\end{array}$ & (not available) \\
\hline $\begin{array}{l}\text { Fluence } \\
\text { (spectrum) }\end{array}$ & $\begin{array}{l}\text { Fluence not available; fast } \\
\text { spectrum }\end{array}$ \\
\hline
\end{tabular}

\section{OTHER FUEL SAMPLE INFORMATION}

FFTF driver fuel pins, wire wrapped; $4.8 \mathrm{~cm}$ Inconel reflector above the two $\mathrm{UO}_{2}$ solid upper insulator pellets; $18.5 \mathrm{~cm}^{3}$ gas plenum volume filled at $1 \mathrm{~atm}$ at room temperature

\begin{tabular}{|l|l|}
\hline \multicolumn{2}{|c|}{ TEST CONDITIONS, MEASUREMENTS, \& ANALYSES } \\
\hline $\begin{array}{l}\text { Coolant } \\
\text { environment }\end{array}$ & Flowing sodium, 103-114 g/s \\
\hline $\begin{array}{l}\text { Transient } \\
\text { conditions }\end{array}$ & $\begin{array}{l}\text { 6-s hold at } 240 \text { W/g; then rise on } 5 \text { c/s TOP } \\
\text { (20.85-s exponential period) to cladding fail ure, } \\
\text { then immediate reactor scram }\end{array}$ \\
\hline $\begin{array}{l}\text { Fuel sample } \\
\text { configuration } \\
\text { in the test }\end{array}$ & Single pin \\
\hline $\begin{array}{l}\text { Test vehicle \& } \\
\text { experiment } \\
\text { system }\end{array}$ & $\begin{array}{l}\text { Single Pin Test Loop (SPTL) - Type B, with } \\
\text { dysprosium collars to axially shape the neutron } \\
\text { flux reaching the test fuel }\end{array}$ \\
\hline $\begin{array}{l}\text { Measurements } \\
\text { /diagnostics } \\
\text { during test }\end{array}$ & $\begin{array}{l}\text { Thermocouples, flow sensors, fuel motion } \\
\text { (hodoscope) }\end{array}$ \\
\hline $\begin{array}{l}\text { Posttest } \\
\text { coolant flow } \\
\text { condition }\end{array}$ & $\begin{array}{l}\text { Incomplete blockage; } 90 \% \text { of initial flow rate, but } \\
\text { the flow tube was breached. }\end{array}$ \\
\hline $\begin{array}{l}\text { Posttest } \\
\text { condition of } \\
\text { test sample(s) }\end{array}$ & $\begin{array}{l}\text { Cladding breach was at } 79 \mathrm{~cm} \text { above fuel bottom } \\
\text { and less than } 1 \mathrm{~cm} \text { long. }\end{array}$ \\
\hline $\begin{array}{l}\text { Posttest } \\
\text { measurements } \\
\text { \& information }\end{array}$ & $\begin{array}{l}\text { Neutron radiography, ceramography, cladding } \\
\text { strain measurement }\end{array}$ \\
\hline $\begin{array}{l}\text { Posttest } \\
\text { analyses }\end{array}$ & $\begin{array}{l}\text { TEMECH thermal-mechanical analysis of fuel, } \\
\text { cladding, and flow tube, including cladding } \\
\text { failure time and location. }\end{array}$ \\
\hline
\end{tabular}

\section{KEY SUMMARY INFORMATION ABOUT THE TEST}

This was the first FFTF-type fuel pin to be transient tested in TREAT. The overpower transient closely matched the fuel heating that would occur in FFTF during a 5-cents/s reactivity insertion. The test goal was to determine the time and location of cladding failure in a lowburnup pin and to provide information for comparing with analytical predictions. Some axial fuel movement occurred $5 \mathrm{~s}$ before the cladding failed. Failure occurred at a fuel height of $84 \%$, at about 3.1 times the nominal power of $41 \mathrm{~kW} / \mathrm{m}$, and resulted in an unusually small cladding breach. Molten fuel moved upward within the fuel pin, pushing the upper insulator pellets and reflector upward enough to fully compress the spring in the pin plenum. Molten fuel that left the cladding penetrated the thin flow tube wall and entered a gas space radially beyond. The relatively-intact condition of the fuel pin after the test allowed detailed examination of the condition of the pin at the time of fail ure. The cladding was nearly penetrated by cracks near the axial elevation of the cladding breach. The fuel that remained within the pin had a large central hole that had apparenty been occupied by molten fuel. The fuel melt radius fraction (r/R) was 0.81 at $X I L-0.25$, 0.86 at $X / L=0.47$ and 0.75 , and 0.75 at $X / L=0.88$.

Test TS-1 was the low-burnup counterpart of medium-burnup-fuel test TS-2. 


\title{
Summary of TREAT Test TS-2
}

\author{
Conducted 11 January 1984
}

Transient No. 2524

\section{PRE-TEST SAMPLE CHARACTERIZATION}

\begin{tabular}{|l|l|}
\hline \multicolumn{2}{|c|}{ FUEL } \\
\hline Form & He-bonded MOX \\
\hline Composition & $\mathrm{UO}_{2}$ (Natural) $-22.4 \mathrm{w} / \mathrm{PuO}_{2}$ \\
\hline Geometry & Solid, dished pellet \\
\hline Fissile height & $91.4 \mathrm{~cm}$ \\
\hline $\begin{array}{l}\text { Smear } \\
\text { density }\end{array}$ & $85.6 \%$ of theoretical \\
\hline
\end{tabular}

\begin{tabular}{|l|l|}
\hline \multicolumn{2}{|c|}{ CLADDING } \\
\hline Material & $20 \%$ cdd-worked type 316 SS \\
\hline OD & $0.584 \mathrm{~cm}$ \\
\hline Thickness & $0.038 \mathrm{~cm}$ \\
\hline
\end{tabular}

\begin{tabular}{|l|l|}
\hline \multicolumn{2}{|l|}{ PRE-IRRADIATION CHARACTERISTICS } \\
\hline BU (peak axial) & $\sim 6 \mathrm{MWd} / \mathrm{kg}$ \\
\hline Peak linear power & $41 \mathrm{~kW} / \mathrm{m} \mathrm{BOL}, 36 \mathrm{~kW} / \mathrm{m} \mathrm{EOL}$ \\
\hline $\begin{array}{l}\text { Peak internal clad } \\
\text { temperature }\end{array}$ & (not available) \\
\hline $\begin{array}{l}\text { Fluence } \\
\text { (spectrum) }\end{array}$ & $\begin{array}{l}\text { Fluence not available; fast } \\
\text { spectrum }\end{array}$ \\
\hline
\end{tabular}

\section{OTHER FUEL SAMPLE INFORMATION}

Wire wrapped; $4.8 \mathrm{~cm}$ Inconel reflector above the two $\mathrm{UO}_{2}$ upper insulator pellets; $18.5 \mathrm{~cm}^{3}$ gas plenum volume filled at $1 \mathrm{~atm}$ at room temperature

\begin{tabular}{|l|l|}
\hline \multicolumn{2}{|c|}{ TEST CONDITIONS, MEASUREMENTS, \& ANALYSES } \\
\hline $\begin{array}{l}\text { Coolant } \\
\text { environment }\end{array}$ & Flowing sodium, 113 to $103 \mathrm{~g} / \mathrm{s}$ \\
\hline $\begin{array}{l}\text { Transient } \\
\text { conditions }\end{array}$ & $\begin{array}{l}\text { 7-s hold at } 34 \mathrm{~kW} / \mathrm{m} \text {; then rise on 20.8-s } \\
\text { exponential period (5 c/s TOP simulation) to } \\
\text { cladding failure; then immediate reactor scram }\end{array}$ \\
\hline $\begin{array}{l}\text { Fuel sample } \\
\text { configuration } \\
\text { in the test }\end{array}$ & Single pin \\
\hline $\begin{array}{l}\text { Test vehicle \& } \\
\text { experiment } \\
\text { system }\end{array}$ & $\begin{array}{l}\text { Single Pin Test Loop (SPTL) - Type B, with } \\
\text { dysprosium collars to axially shape the neutron } \\
\text { flux reaching the test fuel }\end{array}$ \\
\hline $\begin{array}{l}\text { Measurements } \\
\text { /diagnostics } \\
\text { during test }\end{array}$ & $\begin{array}{l}\text { Thermocouples, flow sensors, fuel motion } \\
\text { (hodoscope) }\end{array}$ \\
\hline $\begin{array}{l}\text { Posttest } \\
\text { coolant flow } \\
\text { condition }\end{array}$ & $\begin{array}{l}\text { 10\% of the initial flow rate, but the flowtube wall } \\
\text { had been extensively breached }\end{array}$ \\
\hline $\begin{array}{l}\text { Posttest } \\
\text { condition of } \\
\text { test sample(s) }\end{array}$ & $\begin{array}{l}\text { The fuel pin was destroyed in the fuel region } \\
\text { above X/L = 0.6. }\end{array}$ \\
\hline $\begin{array}{l}\text { Posttest } \\
\text { measurements } \\
\text { \& information }\end{array}$ & $\begin{array}{l}\text { Neutron radiography, posttest examination of } \\
\text { remains }\end{array}$ \\
\hline $\begin{array}{l}\text { Posttest } \\
\text { analyses }\end{array}$ & $\begin{array}{l}\text { TEMECH thermal-mechanical analysis of fuel, } \\
\text { cladding, and flow tube, including cladding } \\
\text { failure time and location. }\end{array}$ \\
\hline
\end{tabular}

KEY SUMMARY INFORMATION ABOUT THE TEST

This was the second FFTF-type fuel pin to be transient tested in TREAT. The overpower transient closely matched the fuel heating that would occur in FFTF during a 5-cents/s reactivity insertion. The test goal was to determine the time and location of cladding fail ure in a medium-burnup pin and to provide information for comparing with analytical predictions. About $2.5 \mathrm{~cm}$ of axial fuel relocation above the original fuel column (apparently fully compressing the spring in the pin plenum) began about $4 \mathrm{~s}$ before the cladding failed. Failure occurred at about 3.4 times the nominal end-of-life power of $36 \mathrm{~kW} / \mathrm{m}$ and resulted in extensive cladding destruction and multiple breaches of the flow tube in the axial region $X L=0.6-0.9$. More than half of the fuel inventory was ejected from the cladding. The radial melt fraction (r/R) of the fuel was $80 \%$ at $X / L=0.25$ and $87 \%$ at $X / L=0.59$.

Test TS-2 was the moderate-burnup-fuel counterpart of low-burnup-fuel test TS-1. 


\section{Appendix G: One-page Summaries of EBT-series Tests}

One-page summaries of each of the EBT-series tests are included here. These summaries can also be found in TREXR [6]. 


\section{Summary of TREAT Test EBT-1 \\ Performed 25 April 1983}

Transient No. 2441

PRE-TEST SAMPLE CHARACTERIZATION
\begin{tabular}{|l|l|}
\hline \multicolumn{2}{|c|}{ FUEL } \\
\hline Form & He-bonded MOX \\
\hline Composition & Pul(U+Pu) $=0.29 ; 81 \%$ enr. U \\
\hline Geometry & Solid pellet; $4.95 \mathrm{~mm}$ diameter \\
\hline Fissile height & $34.3 \mathrm{~cm}$ \\
\hline $\begin{array}{l}\text { Smear } \\
\text { density }\end{array}$ & $90.2 \%$ of theoretical \\
\hline
\end{tabular}

\begin{tabular}{|l|l|}
\hline \multicolumn{2}{|c|}{ CLADDING } \\
\hline Material & D9 alloy, 20\% cold worked \\
\hline OD & $5.84 \mathrm{~mm}$ \\
\hline Thickness & $0.38 \mathrm{~mm}$ \\
\hline
\end{tabular}

\begin{tabular}{|l|l|}
\hline \multicolumn{2}{|l|}{ PRE-IRRADIATION CHARACTERISTICS } \\
\hline BU (peak axial) & $9.4 \%$ of $\mathrm{HM}(90 \mathrm{MWd} / \mathrm{kg})$ \\
\hline Peak linear power & $34.8 \mathrm{~kW} / \mathrm{m}$ \\
\hline $\begin{array}{l}\text { Peak internal clad } \\
\text { temperature }\end{array}$ & (not available) \\
\hline $\begin{array}{l}\text { Fluence } \\
\text { (spectrum) }\end{array}$ & $\begin{array}{l}7.0 \times 10^{22} \mathrm{n} / \mathrm{cm}^{2} \\
\text { fast spectrum }\end{array}$ \\
\hline
\end{tabular}

OTHER FUEL SAMPLE INFORMATION

$65 \mathrm{~g}$ of fuel in the pin.

\begin{tabular}{|l|l|}
\hline \multicolumn{2}{|c|}{ TEST CONDITIONS, MEASUREMENTS, \& ANALYSES } \\
\hline $\begin{array}{l}\text { Coolant } \\
\text { environment }\end{array}$ & Static NaK \\
\hline $\begin{array}{l}\text { Transient } \\
\text { conditions }\end{array}$ & $\begin{array}{l}5.6 \text { s preheat at } ~ 40 \mathrm{~kW} / \mathrm{m} \text {, then } 50 \mathrm{c} / \mathrm{s} \text { reactivity } \\
\text { ramp overpower to } 270 \mathrm{~kW} / \mathrm{m} \text { peak }\end{array}$ \\
\hline $\begin{array}{l}\text { Fuel sample } \\
\text { configuration } \\
\text { in the test }\end{array}$ & Single pin \\
\hline $\begin{array}{l}\text { Test vehicle \& } \\
\text { experiment } \\
\text { system }\end{array}$ & $\begin{array}{l}\text { HUT-series static capsule; with boron thermal } \\
\text { neutron filtering; pin NaK-bonded to } 7.8 \text { mm- } \\
\text { thick nickel circumferential heat sink }\end{array}$ \\
\hline $\begin{array}{l}\text { Measurements } \\
\text { /diagnostics } \\
\text { during test }\end{array}$ & Thermocouples, fuel motion (hodoscope) \\
\hline $\begin{array}{l}\text { Posttest } \\
\text { coolant flow } \\
\text { condition }\end{array}$ & (not applicable) \\
\hline $\begin{array}{l}\text { Posttest } \\
\text { condition of } \\
\text { test sample(s) }\end{array}$ & Massively and extensively disrupted \\
\hline $\begin{array}{l}\text { Posttest } \\
\text { measurements } \\
\text { \& information }\end{array}$ & Neutron radiography, gas sampling \\
\hline $\begin{array}{l}\text { Posttest } \\
\text { analyses }\end{array}$ & (unknown) \\
\hline
\end{tabular}

\section{KEY SUMMARY INFORMATION ABOUT THE TEST}

Test EBT-1 duplicated the same test-fuel thermal conditions as in TREAT Test HUT5-2B (designated as HUT5-1648 in TREXR), which was performed on a 316 SS-clad pin of design similar to the pin in EBT-1 but had only half the burnup. It also duplicated the thermal conditions in test EBT-2, performed on a similar D9-clad pin but of $4.1 \%$ burnup. In EBT-1 the cladding breached at $220 \mathrm{~kW} / \mathrm{m}$ ( five times normal power) near the top of the fuel column, about $0.5 \mathrm{~s}$ before test termination. The major fuel motion occurred within $20 \mathrm{~ms}$ duration and involved fuel expansion above the fuel column and also to the sides of the pin. Fuel was also detected escaping from the cladding in the axial region 0 to $7 \mathrm{~cm}$ above the midplane. Significant fuel expulsion to above the test fuel region occurred, with significant voiding of fuel along the length of the pin. The power at initial cladding breach was $~ 7 \%$ lower than in HUT5-2B and $10 \%$ higher than in EBT-2. The location of initial breach was the same as in HUT5-2B but much higher than the midplane initial breach in EBT-2. 


\section{Summary of TREAT Test EBT-2 \\ Performed 19 April 1983}

Transient No. 2438

PRE-TEST SAMPLE CHARACTERIZATION
\begin{tabular}{|l|l|}
\hline \multicolumn{2}{|c|}{ FUEL } \\
\hline Form & He-bonded MOX; \\
\hline Composition & Pu/(U+Pu) $=0.29 ; 81 \%$ enr. U \\
\hline Geometry & Solid pellet; $4.95 \mathrm{~mm}$ diameter \\
\hline Fissile height & $34.3 \mathrm{~cm}$ \\
\hline $\begin{array}{l}\text { Smear } \\
\text { density }\end{array}$ & $90.2 \%$ of theoretical \\
\hline
\end{tabular}

\begin{tabular}{|l|l|}
\hline \multicolumn{2}{|c|}{ CLADDING } \\
\hline Material & D9 alloy, 20\% cold worked \\
\hline OD & $5.84 \mathrm{~mm}$ \\
\hline Thickness & $0.38 \mathrm{~mm}$ \\
\hline
\end{tabular}

\begin{tabular}{|l|l|}
\hline \multicolumn{2}{|l|}{ PRE-IRRADIATION CHARACTERISTICS } \\
\hline BU (peak axial) & $\sim 4.2 \%$ of $\mathrm{HM}$ \\
\hline Peak linear power & $35 \mathrm{~kW} / \mathrm{m}$ \\
\hline $\begin{array}{l}\text { Peak internal clad } \\
\text { temperature }\end{array}$ & (not available) \\
\hline $\begin{array}{l}\text { Fluence } \\
\text { (spectrum) }\end{array}$ & $\begin{array}{l}3.1 \times 10^{22} \mathrm{n} / \mathrm{cm}^{2} \\
\text { fast spectrum }\end{array}$ \\
\hline
\end{tabular}

OTHER FUEL SAMPLE INFORMATION

$65 \mathrm{~g}$ of fuel in the pin.

\begin{tabular}{|l|l|}
\hline \multicolumn{2}{|c|}{ TEST CONDITIONS, MEASUREMENTS, \& ANALYSES } \\
\hline $\begin{array}{l}\text { Coolant } \\
\text { environment }\end{array}$ & Static NaK \\
\hline $\begin{array}{l}\text { Transient } \\
\text { conditions }\end{array}$ & $\begin{array}{l}5.6 \text { s preheat at } ~ 40 \mathrm{~kW} / \mathrm{m}, \text { then } 50 \mathrm{c} / \mathrm{s} \text { reactivity } \\
\text { ramp overpower to } 270 \mathrm{~kW} / \mathrm{m} \text { peak }\end{array}$ \\
\hline $\begin{array}{l}\text { Fuel sample } \\
\text { configuration } \\
\text { in the test }\end{array}$ & Single pin \\
\hline $\begin{array}{l}\text { Test vehicle \& } \\
\text { experiment } \\
\text { system }\end{array}$ & $\begin{array}{l}\text { HUT-series static capsule; with boron thermal } \\
\text { neutron filtering; pin NaK-bonded to } 7.8 \mathrm{~mm}- \\
\text { thick nickel circumferential heat sink }\end{array}$ \\
\hline $\begin{array}{l}\text { Measurements } \\
\text { /diagnostics } \\
\text { during test }\end{array}$ & Thermocouples, fuel motion (hodoscope) \\
\hline $\begin{array}{l}\text { Posttest } \\
\text { coolant flow } \\
\text { condition }\end{array}$ & (not applicable) \\
\hline $\begin{array}{l}\text { Posttest } \\
\text { condition of } \\
\text { test sample(s) }\end{array}$ & $\begin{array}{l}\text { Greatly disrupted over most of the fuel column } \\
\text { length }\end{array}$ \\
\hline $\begin{array}{l}\text { Posttest } \\
\text { measurements } \\
\text { \& information }\end{array}$ & Neutron radiography, gas sampling \\
\hline $\begin{array}{l}\text { Posttest } \\
\text { analyses }\end{array}$ & \begin{tabular}{l} 
(unknown) \\
\hline
\end{tabular} \\
\hline
\end{tabular}

\section{KEY SUMMARY INFORMATION ABOUT THE TEST}

Test EBT-2 duplicated the same test-fuel thermal conditions as in TREAT Test HUT5-2B (designated as HUT5-1648 in TREXR), which was performed on a 316SS-clad pin of design and burnup similar to the pin in EBT-2. It also duplicated the thermal conditions in test EBT-1, performed on a similar D9-clad pin but of $9.4 \%$ burnup. In EBT-2 the cladding breached at $200 \mathrm{~kW} / \mathrm{m}$ ( five times normal power) near the fuel midplane, about $0.5 \mathrm{~s}$ before test termination. The major fuel motion occurred within 10 ms duration near the fuel midplane, accompanied by fuel loss from the top $20 \%$ and bottom $20 \%$ of the fuel column. About $0.25 \mathrm{~s}$ later, fuel suddenly moved from the midplane to lower regions of the fuel pin. The power at initial cladding breach was $\sim 18 \%$ lower than in HUT5-2B and $10 \%$ lower than in EBT-1. The midplane location of first cladding breach differed significantly from the near-top of fuel locations of initial breach in HUT5-2B and EBT-1. 


\section{Summary of TREAT Test EBT-3 \\ Performed 23 February 1984 \\ Transient No. 2543}

PRE-TEST SAMPLE CHARACTERIZATION
\begin{tabular}{|l|l|}
\hline \multicolumn{2}{|c|}{ FUEL } \\
\hline Form & $\mathrm{He}$-bonded MOX \\
\hline Composition & $\begin{array}{l}\text { 1: } \mathrm{UO}_{2}-25.0 \mathrm{PuO}_{2} \\
2: \mathrm{UO}_{2}-29.3 \mathrm{PuO}_{2} \\
\end{array}$ \\
$\begin{array}{ll}\text { 3: } \mathrm{UO}_{2}-29.3 \mathrm{PuO}_{2} \\
\text { All Pu/(Pu+U) }=0.25\end{array}$ \\
\hline Geometry & Solid pellet \\
\hline Fissile height & $34.3 \mathrm{~cm}$ \\
\hline $\begin{array}{l}\text { Smear } \\
\text { density }\end{array}$ & $\sim 86 \%$ of theoretical \\
\hline
\end{tabular}

\begin{tabular}{|c|c|}
\hline \multicolumn{2}{|r|}{ CLADDING } \\
\hline Material & $\begin{array}{l}\text { 1: } 316 \text { SS, } 20 \% \text { cold worked } \\
\text { 2: D9 austenitic alloy, } 20 \% \mathrm{CW} \\
\text { 3: HT9 ferritic alloy, air quenched, } \\
\text { tempered }\end{array}$ \\
\hline OD & $5.84 \mathrm{~mm}$ \\
\hline Thickness & $0.38 \mathrm{~mm}$ \\
\hline
\end{tabular}

\begin{tabular}{|l|l|}
\hline \multicolumn{2}{|c|}{ TEST CONDITIONS, MEASUREMENTS, \& ANALYSES } \\
\hline $\begin{array}{l}\text { Coolant } \\
\text { environment }\end{array}$ & Flowing sodium \\
\hline $\begin{array}{l}\text { Transient } \\
\text { conditions }\end{array}$ & $\begin{array}{l}\text { 6-s steady state of between } 35-38 \mathrm{~kW} / \mathrm{m} \text {, then } \\
\text { at } 10 \text { s from the start of the overpower, then } \\
\text { scram }\end{array}$ \\
\hline $\begin{array}{l}\text { Fuel sample } \\
\text { configuration } \\
\text { in the test }\end{array}$ & Three pins, each in a separate flowtube \\
\hline $\begin{array}{l}\text { Test vehicle \& } \\
\text { experiment } \\
\text { system }\end{array}$ & $\begin{array}{l}\text { Mark-IIC sodium loop with axial thermal neutron } \\
\text { filters }\end{array}$ \\
\hline $\begin{array}{l}\text { Measurements } \\
\text { /diagnostics } \\
\text { during test }\end{array}$ & $\begin{array}{l}\text { Flowmeters, thermocouples, pressure } \\
\text { transducers, fuel motion monitor (hodoscope) }\end{array}$ \\
\hline $\begin{array}{l}\text { Posttest } \\
\text { coolant flow } \\
\text { condition }\end{array}$ & No blockages \\
\hline $\begin{array}{l}\text { Posttest } \\
\text { condition of } \\
\text { test sample(s) }\end{array}$ & $\begin{array}{l}\text { No cladding failures, fuel melting in all pins, } \\
\text { maximum strain 0.7\% (in the HT9-clad pin) }\end{array}$ \\
\hline $\begin{array}{l}\text { Posttest } \\
\text { measurements } \\
\text { \& information }\end{array}$ & $\begin{array}{l}\text { Neutron radiography, macro examination, } \\
\text { profilometry, ceramography }\end{array}$ \\
\hline $\begin{array}{l}\text { Posttest } \\
\text { analyses }\end{array}$ & TMECH and SIEX code analyses \\
\hline
\end{tabular}

\begin{tabular}{|l|l|}
\hline \multicolumn{2}{|l|}{ PRE-IRRADIATION CHARACTERISTICS } \\
\hline BU (peak axial) & 82 to $92 \mathrm{MWd} / \mathrm{kg}$ \\
\hline Peak linear power & $1: 38 \mathrm{~kW} / \mathrm{m}$ \\
& $2: 37 \mathrm{~kW} / \mathrm{m}$ \\
& $3: 36 \mathrm{~kW} / \mathrm{m}$ \\
\hline Peak internal clad & (not available) \\
temperature & \\
\hline Fluence & $\sim 6.3 \times 10^{2} \mathrm{n}^{\mathrm{cm}} \mathrm{cm}^{2}$ \\
(spectrum) & (fast spectrum) \\
\hline
\end{tabular}

\section{KEY SUMMARY INFORMATION ABOUT THE TEST}

Test EBT-3 was conducted to compare the effects of a hypothetical unprotected LMFBR transient overpower accident on three fuel pins with different types of cladding irradiated to near-nominal exposure goals. Test conditions were established to allow all three pins to fail, but no failures occurred. It is believed that axial extrusion of molten fuel into the solid-insulatorpellet area served as a pressure relief mechanism for the central cavity. The peak power levels reached in the pins (in terms of multiples of their prior steady-state irradiation power) were 2.9 for the HT9-clad pin, 2.6 for the D9-clad pin, and 2.4 for the 316 SS-clad pin. The $0.7 \%$ local peaking in cladding strain in the HT9-clad pin was just above the top of the fuel and apparently due to movement of molten fuel slightly above the fuel column. The peak strain elsewhere in that pin was $0.3 \%$ and in the other two pins was $0.1 \%$. The central void in each pin was filled discontinuously by once-molten fuel, and the maximum areal melt fraction was about $36 \%$. 


\title{
Summary of TREAT Test EBTB
}

\author{
Performed 12 March 1984
}

Transient No. 2550

\section{PRE-TEST SAMPLE CHARACTERIZATION}

\begin{tabular}{|l|l|}
\hline \multicolumn{2}{|c|}{ FUEL } \\
\hline Form & He-bonded $\mathrm{MOX}$ \\
\hline Composition & $\left(96 \% \mathrm{UO}_{2,} 4 \% \mathrm{PuO}_{2}\right)$ \\
\hline Geometry & Solid dished pellets \\
\hline Fissile height & $34.4 \mathrm{~cm}$ \\
\hline $\begin{array}{l}\text { Smear } \\
\text { density }\end{array}$ & $95 \%$ of theoretical \\
\hline
\end{tabular}

\begin{tabular}{|l|l|}
\hline \multicolumn{2}{|c|}{ CLADDING } \\
\hline Material & $316 \mathrm{SS}, 20 \%$ cold-worked \\
\hline OD & $12.85 \mathrm{~mm}$ \\
\hline Thickness & $0.38 \mathrm{~mm}$ \\
\hline
\end{tabular}

\begin{tabular}{|l|l|}
\hline PRE-IRRADIATION CHARACTERISTICS \\
\hline BU (peak axial) & $3 \%$ \\
\hline Peak linear power & $\begin{array}{l}\text { (see "Other Fuel Sample } \\
\text { Information" below) }\end{array}$ \\
\hline $\begin{array}{l}\text { Peak internal clad } \\
\text { temperature }\end{array}$ & (not available) \\
\hline $\begin{array}{l}\text { Fluence } \\
\text { (spectrum) }\end{array}$ & $\begin{array}{l}1.0 \times 10^{23} \mathrm{n} / \mathrm{cm}^{2} \\
\text { (fast spectrum) }\end{array}$ \\
\hline
\end{tabular}

\section{OTHER FUEL SAMPLE INFORMATION}

The test pin had been previously irradiated at steady state, which generated $3 \%$ peak cladding strain. It was then subjected to a load-following and slow overpower test in EBR-II.

\begin{tabular}{|l|l|}
\hline \multicolumn{2}{|c|}{ TEST CONDITIONS, MEASUREMENTS, \& ANALYSES } \\
\hline $\begin{array}{l}\text { Coolant } \\
\text { environment }\end{array}$ & Flowing sodium \\
\hline $\begin{array}{l}\text { Transient } \\
\text { conditions }\end{array}$ & $\begin{array}{l}\text { 7-s preheat at low power, then } \\
\text { ended by scram on flow signal } \\
\sim 8 \text { s later }\end{array}$ \\
\hline $\begin{array}{l}\text { Fuel sample } \\
\text { configuration } \\
\text { in the test }\end{array}$ & Single pin \\
\hline $\begin{array}{l}\text { Test vehicle \& } \\
\text { experiment } \\
\text { system }\end{array}$ & $\begin{array}{l}\text { Mark-IIC sodium loop, with dysprosium collars to } \\
\text { prevent power peaking at the ends of the blanket } \\
\text { fuel column }\end{array}$ \\
\hline $\begin{array}{l}\text { Measurements } \\
\text { /diagnostics } \\
\text { during test }\end{array}$ & $\begin{array}{l}\text { Thermocouples, flow meters, fuel motion monitor } \\
\text { (hodoscope) }\end{array}$ \\
\hline $\begin{array}{l}\text { Posttest } \\
\text { coolant flow } \\
\text { condition }\end{array}$ & Complete blockage \\
\hline $\begin{array}{l}\text { Posttest } \\
\text { condition of } \\
\text { test sample(s) }\end{array}$ & $\begin{array}{l}\text { Extensive damage, especially over the top half } \\
\text { of the blanket fuel region }\end{array}$ \\
\hline $\begin{array}{l}\text { Posttest } \\
\text { measurements } \\
\text { \& information }\end{array}$ & $\begin{array}{l}\text { Neutron radiography, gamma scanning, } \\
\text { destructive examination, macrophotography }\end{array}$ \\
\hline $\begin{array}{l}\text { Posttest } \\
\text { analyses }\end{array}$ & \begin{tabular}{l} 
LIFE-4 \\
\hline
\end{tabular} \\
\hline
\end{tabular}

\section{KEY SUMMARY INFORMATION ABOUT THE TEST}

Test EBTB was performed to determine the ability of MOX blanket fuel rod to withstand an unprotected $50 \phi / s$ fast reactor overpower transient. The cladding breached when the power level in the pin reached $300 \mathrm{~kW} / \mathrm{m}(600 \%$ overpower compared to the pin's steady state irradiation power). Molten fuel expelled from the pin caused a sharp sodium flow anomaly, which triggered a reactor scram as planned. A postfailure scenario most consistent with the data is that the cladding first breached by near the fuel midplane. Molten fuel expelled through the breach was swept upward in the coolant channel to the top of the fuel region where it collected, formed a blockage, and melted through the flowtube wall. The resulting reduced cooling of the above-midplane part of the pin caused gross overheating, melting, and destruction of that part of the fuel column. There was no breach in the flowtube at the midplane. Where pellets remained relatively intact, there were no changes in blanket fuel structure nor in the fuel cracking pattern due to the transient. The initial cladding failure was deduced to be due to fuelcladding mechanical interaction. The areal melt fraction of the fuel was $26 \%$ at $x / L=0.13, \sim 60 \%$ at $x / L=0.5$, and $85 \%$ at $\mathrm{x} / \mathrm{L}=0.86$. Channel blockage was present along almost the entire upper half of the fuel column. 


\section{Appendix H: One-page Summaries of L-series Tests}

One-page summaries of each of the L-series tests are included here. These summaries can also be found in TREXR [6]. 


\title{
Summary of TREAT Test L1
}

\author{
Performed 15 September 1970
}

Transient Nos. 1136, 1137, and 1138

\section{PRE-TEST SAMPLE CHARACTERIZATION}

\begin{tabular}{|l|l|}
\hline \multicolumn{2}{|c|}{ FUEL } \\
\hline Form & He-bonded MOX \\
\hline Composition & $\begin{array}{l}\mathrm{UO}_{2}-75 \% \mathrm{PuO}_{2} \text {, with the } \mathrm{UO}_{2} \text { being } \\
65 \% \text { enriched }\end{array}$ \\
\hline Geometry & Solid pellet \\
\hline Fissile height & $34.3 \mathrm{~cm}$ \\
\hline $\begin{array}{l}\text { Smear } \\
\text { density }\end{array}$ & $\begin{array}{l}\text { Unknown, but probably } 87 \% \text { of } \\
\text { theoretical }\end{array}$ \\
\hline
\end{tabular}

\begin{tabular}{|l|l|}
\hline \multicolumn{2}{|c|}{ CLADDING } \\
\hline Material & SS 316 $(20 \% \mathrm{CW})$ \\
\hline OD & $5.84 \mathrm{~mm}$ \\
\hline Thickness & $0.38 \mathrm{~mm}$ \\
\hline
\end{tabular}

\begin{tabular}{|l|l|}
\hline \multicolumn{2}{|l|}{ PRE-IRRADIATION CHARACTERISTICS } \\
\hline BU (peak axial) & None \\
\hline Peak linear power & (not applicable) \\
\hline $\begin{array}{l}\text { Peak internal clad } \\
\text { temperature }\end{array}$ & (not applicable) \\
\hline $\begin{array}{l}\text { Fluence } \\
\text { (spectrum) }\end{array}$ & (none) \\
\hline
\end{tabular}

\section{OTHER FUEL SAMPLE INFORMATION}

The fuel pin was spiral-wrapped with a $1 \mathrm{~mm}$ diameter spacer wire.

\begin{tabular}{|l|l|}
\hline \multicolumn{2}{|c|}{ TEST CONDITIONS, MEASUREMENTS, \& ANALYSES } \\
\hline $\begin{array}{l}\text { Coolant } \\
\text { environment }\end{array}$ & $\begin{array}{l}\text { Flowing sodium, set at } 2.35 \mathrm{~m} / \mathrm{s} \text { pretest in the } \\
\text { first transient, } \sim 3.8 \mathrm{~m} / \mathrm{s} \text { in the second transient; } \\
\text { unknown in the third transient }\end{array}$ \\
\hline $\begin{array}{l}\text { Transient } \\
\text { conditions }\end{array}$ & $\begin{array}{l}\text { Three } 12-15 \mathrm{~s} \text { duration power transients, each } \\
\text { intended to produce pin power } ~ 15 \% \text { higher than } \\
\text { nominal. }\end{array}$ \\
\hline $\begin{array}{l}\text { Fuel sample } \\
\text { configuration } \\
\text { in the test }\end{array}$ & $\begin{array}{l}\text { Single pin geometry, surrounded by a flowtube } \\
\text { with 1.2-mm clearance between pin cladding and } \\
\text { flowtube wall }\end{array}$ \\
\hline $\begin{array}{l}\text { Test vehicle \& } \\
\text { experiment } \\
\text { system }\end{array}$ & Mark-IIA integral sodium loop \\
\hline $\begin{array}{l}\text { Measurements } \\
\text { /diagnostics } \\
\text { during test }\end{array}$ & $\begin{array}{l}\text { Thermocouples, flowmeters, pressure } \\
\text { transducers, and the fast-neutron hodoscope } \\
\text { fuel motion detector. }\end{array}$ \\
\hline $\begin{array}{l}\text { Posttest } \\
\text { coolant flow } \\
\text { condition }\end{array}$ & No evidence of permanent flow blockage \\
\hline $\begin{array}{l}\text { Posttest } \\
\text { condition of } \\
\text { test sample(s) }\end{array}$ & $\begin{array}{l}\text { Fuel pin was highly warped but cladding } \\
\text { remained unbreached. Evidence of localized } \\
\text { incipient cladding melting and buldging just } \\
\text { below the top of the fuel column. }\end{array}$ \\
\hline $\begin{array}{l}\text { Posttest } \\
\text { measurements } \\
\text { \& information }\end{array}$ & $\begin{array}{l}\text { Neutron radiography, eddy current examination } \\
\text { of the cladding, cladding ovality measurement }\end{array}$ \\
\hline $\begin{array}{l}\text { Posttest } \\
\text { analyses }\end{array}$ & Analyses using SAS1A \\
\hline
\end{tabular}

\section{KEY SUMMARY INFORMATION ABOUT THE TEST}

Test L1 was kept relatively simple because it was the first test to explore the ability of the sodium loop to create loss-of-flow simulations in assemblies of sodium-coded FFTF-type fuel pins. Thus, the test fuel was not pre-irradiated, and it was planned to approach, but not exceed, the cladding failure threshdd of the fuel pin when operating at $15 \%$ over design power. Programmed flow coastdown was not available, so successively more-severe power-to-flow mismatch conditions were created by subjecting the fued pin to three power transients, each producing about $15 \%$ overpower in the test pin, but using successively-ower constant sodium flow rate from one transient to the next.

During the first transient, as the TREAT power was rising to the flattop level, the inlet sodium flow quickly decreased by $\sim 40 \%$ (where it remained for the rest of the power transient), the outlet flow rate decreased by $\sim 20 \%$, and the hodoscope detected movement of testfuel pellets within the cladding. Thereafter, the outet flow signal rose steadily, rising to $\sim 2.5$ times the initial flow signal by the end of the power transient. This unexpected behavior was possibly caused by local sodium boiling where the wire wrap and adjacent thermocouple contacted the cladding, which would have caused much of the flow channel to be voided during the transient. By the end of the transient, the fuel pin had become permanently warped, with the distortion reaching the limit allowed by the restraining wall of the surrounding test section, and all of the instrument signals had returned to their pretransient levels.

The second transient initially overshot the intended power level by $60 \%$ and then gradually reached the intended level by the end of the transient. When the power peaked, the inlet and outlet flow rates both suddenly dropped by more than half. The inlet flow rate remained at that value until the end of the power transient when it abruptly returned to its initial level. The outlet flow signal stayed at its $50 \%$ level for about $2 \mathrm{~s}$, then steadily rose to twice its initial value by the end of the power transient. [No flow signals were recorded during the third transient, and the impact of the transient is unknown.] 


\section{Summary of TREAT Test L2}

Performed 28 July 1972

Transient No. 1464

\section{PRE-TEST SAMPLE CHARACTERIZATION}

\begin{tabular}{|l|l|}
\hline \multicolumn{2}{|c|}{ FUEL } \\
\hline Form & He-bonded $\mathrm{MOX}$ \\
\hline Composition & $\begin{array}{l}\mathrm{UO}_{2}-75 \% \mathrm{PuO}_{2}, \text { with the } \mathrm{UO}_{2} \text { being } \\
65 \% \text { enriched }\end{array}$ \\
\hline Geometry & Solid pellet; dished ends \\
\hline Fissile height & $34.3 \mathrm{~cm}$ \\
\hline $\begin{array}{l}\text { Smear } \\
\text { density }\end{array}$ & $87 \%$ of theoretical \\
\hline
\end{tabular}

\begin{tabular}{|l|l|}
\hline \multicolumn{2}{|c|}{ CLADDING } \\
\hline Material & SS $316(20 \% \mathrm{CW})$ \\
\hline OD & $5.84 \mathrm{~mm}$ \\
\hline Thickness & $0.38 \mathrm{~mm}$ \\
\hline
\end{tabular}

\begin{tabular}{|l|l|}
\hline \multicolumn{2}{|l|}{ PRE-IRRADIATION CHARACTERISTICS } \\
\hline BU (peak axial) & None \\
\hline Peak linear power & (not applicable) \\
\hline $\begin{array}{l}\text { Peak internal clad } \\
\text { temperature }\end{array}$ & (not applicable) \\
\hline $\begin{array}{l}\text { Fluence } \\
\text { (spectrum) }\end{array}$ & (none) \\
\hline
\end{tabular}

\section{OT HER FUEL SAMPLE INFORMATION}

The fuel pin was spiral-wrapped with a $1 \mathrm{~mm}$ diameter spacer wire. The plenum pressure was $1 \mathrm{~atm}$ at room temperature. Above the fuel column were $1.27 \mathrm{~cm}$ of $\mathrm{UO}_{2}$ insulator pellets with a $15 \mathrm{~cm}$-long Inconel reflector rod above it.

\begin{tabular}{|l|l|}
\hline \multicolumn{2}{|c|}{ TEST CONDITIONS, MEASUREMENTS, \& ANALYSES } \\
\hline $\begin{array}{l}\text { Coolant } \\
\text { environment }\end{array}$ & $\begin{array}{l}\text { Flowing sodium; programmed reduction in flow } \\
\text { rate down to 10\% in 9.5 s during power transient }\end{array}$ \\
\hline $\begin{array}{l}\text { Transient } \\
\text { conditions }\end{array}$ & $\begin{array}{l}\text { Essential constant power of } ~ 36 \mathrm{~kW} / \mathrm{m} \text { (pin- } \\
\text { bundle average) for 23 s }\end{array}$ \\
\hline $\begin{array}{l}\text { Fuel sample } \\
\text { configuration } \\
\text { in the test }\end{array}$ & $\begin{array}{l}\text { 7-pin bundle of wire-wrapped pins inside a fluted } \\
\text { flow tube }\end{array}$ \\
\hline $\begin{array}{l}\text { Test vehicle \& } \\
\text { experiment } \\
\text { system }\end{array}$ & $\begin{array}{l}\text { Mark-II integral sodium loop with axial flux- } \\
\text { shaping collars (axial max/avg power }=1.08) \\
\text { test train with argon gas space radially } \\
\text { surrounding the flow tube }\end{array}$ \\
\hline $\begin{array}{l}\text { Measurements } \\
\text { /diagnostics } \\
\text { during test }\end{array}$ & $\begin{array}{l}\text { Temperatures, coolant flow rates, coolant } \\
\text { pressures, sodium void detection, fuel motion } \\
\text { monitoring by the fast neutron hodoscope }\end{array}$ \\
\hline $\begin{array}{l}\text { Posttest } \\
\text { coolant flow } \\
\text { condition }\end{array}$ & $\begin{array}{l}\text { Complete flow blockages near bottom and top of } \\
\text { test fuel region }\end{array}$ \\
\hline $\begin{array}{l}\text { Posttest } \\
\text { condition of } \\
\text { test sample(s) }\end{array}$ & $\begin{array}{l}\text { Highly disrupted; with much fuel and cladding } \\
\text { melting, but also with some fuel pellets unmelted } \\
\text { or partially melted. Some melted fuel contained } \\
\text { steel particles. }\end{array}$ \\
\hline $\begin{array}{l}\text { Posttest } \\
\text { measurements } \\
\text { \& information }\end{array}$ & $\begin{array}{l}\text { Neutron and X-ray radiography, destructive } \\
\text { examination of the remains }\end{array}$ \\
\hline $\begin{array}{l}\text { Posttest } \\
\text { analyses }\end{array}$ & Analyses using the SAS2B code. \\
\hline
\end{tabular}

\footnotetext{
KEY SUMMARY INFORMATION ABOUT THE TEST

In Test L2, a preliminary checkout/calibration heat-balance transient was run with nominal sodium flow rate. A second preliminary transient was run with lower flow rate to check the flow-coastdown capability of the loop and to approach but not exceed the cladding failure threshold of the fuel pin.. A structural anomaly found prior to the final transient was identified posttest distorted the flow distribution among the several fuel pins but apparently did not have had much effect at lower flow rates during the experiment. Flow oscillations at the outlet began toward the end of the flow coastdown, indicating sodium boiling, voiding, and then flow chugging. Temperatures suggesting dryout were reached about $2 \mathrm{~s}$ later. The first upward flow eructation was believed to have caused the posttest-observed upward relocation of the plenum section of the fuel elements, upward movement of the reflector rods and top insulator pellets, and compression of the plenum spring. Within $2 \mathrm{~s}$ thereafter, the flow channel was completely plugged. At about this time, the upper third of the fuel columns slumped non-uniformly and non-coherently, with fuel initially spilling out of the cladding at $\sim x / L=0.75$. This was followed by a quiescent period of about $4 \mathrm{~s}$, ending in two large movements of fuel in both directions away from the midplane area. Little or no fuel passed above or below the original fuel region. The lower flow blockage was complete only over a distance of about $1 \mathrm{~cm}$. It was composed mostly of solidified steel surrounding partially intact fuel pellets. The flow blockage at the top of the fuel column was about 4 $\mathrm{cm}$ long, porous, and capped a thin layer of steel. Some of the steel apparently had vaporized.
} 


\section{Summary of TREAT Test L3}

Performed 2 January 1973

Transient No. 1483

\section{PRE-TEST SAMPLE CHARACTERIZATION}

\begin{tabular}{|l|l|}
\hline \multicolumn{2}{|c|}{ FUEL } \\
\hline Form & He-bonded $\mathrm{MOX}$ \\
\hline Composition & $\begin{array}{l}\mathrm{UO}_{2}-75 \% \mathrm{PuO}_{2}, \text { with the } \mathrm{UO}_{2} \text { being } \\
62 \% \text { enriched }\end{array}$ \\
\hline Geometry & Solid pellet; dished ends \\
\hline Fissile height & $34.3 \mathrm{~cm}$ \\
\hline $\begin{array}{l}\text { Smear } \\
\text { density }\end{array}$ & $87.5 \%$ of theoretical \\
\hline
\end{tabular}

\begin{tabular}{|l|l|}
\hline \multicolumn{2}{|c|}{ CLADDING } \\
\hline Material & SS $316(20 \% \mathrm{CW})$ \\
\hline OD & $5.84 \mathrm{~mm}$ \\
\hline Thickness & $0.38 \mathrm{~mm}$ \\
\hline
\end{tabular}

\begin{tabular}{|l|l|}
\hline \multicolumn{2}{|l|}{ PRE-IRRADIATION CHARACTERISTICS } \\
\hline BU (peak axial) & 3.5 at.\% \\
\hline Peak linear power & $\begin{array}{l}30 \mathrm{~kW} / \mathrm{m} \text { initially; then } 38 \\
\mathrm{~kW} / \mathrm{m}\end{array}$ \\
\hline $\begin{array}{l}\text { Peak internal clad } \\
\text { temperature }\end{array}$ & (not available) \\
\hline $\begin{array}{l}\text { Fluence } \\
\text { (spectrum) }\end{array}$ & $\begin{array}{l}\text { Fluence not available; fast } \\
\text { spectrum }\end{array}$ \\
\hline
\end{tabular}

\section{OT HER FUEL SAMPLE INFORMATION}

Each fuel pin was spiral-wrapped with a $1 \mathrm{~mm}$ diameter spacer wire. Plenum pressure was $1 \mathrm{~atm}$ at room temperature. Above the fuel column were $1.27 \mathrm{~cm}$ of $\mathrm{UO}_{2}$ insulator pellets with a $15 \mathrm{~cm}$ spacer tube above it. The irradiated fuel had no central void and $~ 51 \%$ fission gas retention.

\begin{tabular}{|l|l|}
\hline \multicolumn{2}{|c|}{ TEST CONDITIONS, MEASUREMENTS, \& ANALYSES } \\
\hline $\begin{array}{l}\text { Coolant } \\
\text { environment }\end{array}$ & $\begin{array}{l}\text { Flowing sodium; programmed reduction in flow } \\
\text { rate beginning about 2 s after the start of the } \\
\text { constant-power part of the transient, decreasing } \\
\text { to } 10 \% \text { of the initial value within 5 s }\end{array}$ \\
\hline $\begin{array}{l}\text { Transient } \\
\text { conditions }\end{array}$ & $\begin{array}{l}\text { Power rise for short preheat, then power } \\
\text { constant at 30 kW/m for 27 s, then scram }\end{array}$ \\
\hline $\begin{array}{l}\text { Fuel sample } \\
\text { configuration } \\
\text { in the test }\end{array}$ & $\begin{array}{l}\text { 7-pin bundle of wire-wrapped pins inside a fluted } \\
\text { flow tube }\end{array}$ \\
\hline $\begin{array}{l}\text { Test vehicle \& } \\
\text { experiment } \\
\text { system }\end{array}$ & $\begin{array}{l}\text { Mark-IIA integral sodium loop with axial flux- } \\
\text { shaping collars (axial max/avg power }=1.08) \\
\text { test train with argon gas space radially } \\
\text { surrounding the flow tube }\end{array}$ \\
\hline $\begin{array}{l}\text { Measurements } \\
\text { diagnostics } \\
\text { during test }\end{array}$ & $\begin{array}{l}\text { Temperatures, coolant flow rates, coolant } \\
\text { pressures, fuel motion monitoring by the fast } \\
\text { neutron hodoscope }\end{array}$ \\
\hline $\begin{array}{l}\text { Posttest } \\
\text { coolant flow } \\
\text { condition }\end{array}$ & Complete blockage of flow channels. \\
\hline $\begin{array}{l}\text { Posttest } \\
\text { condition of } \\
\text { test sample(s) }\end{array}$ & $\begin{array}{l}\text { Highly disrupted fuel pins, with extensive } \\
\text { removal of fuel from the mid-region of the fue } \\
\text { column and upward displacement of fuel into } \\
\text { region above the original fuel zone }\end{array}$ \\
\hline $\begin{array}{l}\text { Posttest } \\
\text { measurements } \\
\text { \& information }\end{array}$ & $\begin{array}{l}\text { Neutron and X-ray radiography, destructive } \\
\text { examination of the remains }\end{array}$ \\
\hline $\begin{array}{l}\text { Posttest } \\
\text { analyses }\end{array}$ & Analyses by SAS2A, THTE \\
\hline
\end{tabular}

\section{KEY SUMMARY INFORMATION ABOUT THE TEST}

Test L3 was the first test of preirradiated FFTF-type fuel in a loss-of-flow simulation. Early in the transient, lateral squirming of the fuel region began and continued for the first half of the constant-power part of the transient. Failure of the fluted flowtube appeared (from thermocouple failures) to occur about half-way through the transient. Fuel motion continued simultaneous with additional melting of the flowtube by molten fuel. There was a marked loss of fuel from the axial center part of the fuel region with upward fuel movement. About $4 \mathrm{~s}$ before scram, the central part of the fuel region suddenly voided within $150 \mathrm{~ms}$, with about $10 \%$ of the fuel moving upward slightly beyond the original fuel zone. Lesser fuel motions continued until scram. There was no indication of outlet flow chugging as had been observed in Test L2 on fresh fuel. By the end of the test, a blockage consisting of melted steel around the fuel elements was present at the extreme bottom of the original fuel region. Just above that blockage the fuel pellets appeared to have remained unmelted. Additional melted steel was present below the blockage. A partial blockage of molten steel and porous melted fuel had been formed at the top of the original fuel region. Along the original fuel region, most of the fluted flowtube had melted away, and fuel that was present was no longer in pellet form -except at the extreme ends of that region where large masses of melted fuel had accumulated around partially-intact fuel pellets. There was little penetration of molten steel into the fuel. Bulk melted fuel had areal porosity in the $30-40 \%$ range. No evidence was found to indicate that the tops of the fuel pins might have moved up or down as a unit. 


\section{Summary of TREAT Test L4}

Performed 23 March 1973

Transient No. 1503

\section{PRE-TEST SAMPLE CHARACTERIZATION}

\begin{tabular}{|l|l|}
\hline \multicolumn{2}{|c|}{ FUEL } \\
\hline Form & He-bonded $\mathrm{MOX}$ \\
\hline Composition & $\begin{array}{l}\mathrm{UO}_{2}-75 \% \mathrm{PuO}_{2}, \text { with the } \mathrm{UO}_{2} \text { being } \\
77 \% \text { enriched }\end{array}$ \\
\hline Geometry & Solid pellet \\
\hline Fissile height & $34.3 \mathrm{~cm}$ \\
\hline $\begin{array}{l}\text { Smear } \\
\text { density }\end{array}$ & $85.0 \%$ of theoretical \\
\hline
\end{tabular}

\begin{tabular}{|l|l|}
\hline \multicolumn{2}{|c|}{ CLADDING } \\
\hline Material & SS $316(20 \% \mathrm{CW})$ \\
\hline OD & $5.84 \mathrm{~mm}$ \\
\hline Thickness & $0.38 \mathrm{~mm}$ \\
\hline
\end{tabular}

\begin{tabular}{|l|l|}
\hline \multicolumn{2}{|l|}{ PRE-IRRADIATION CHARACTERISTICS } \\
\hline BU (peak axial) & $4.3 \mathrm{at} \%$ \\
\hline Peak linear power & $\begin{array}{l}30 \mathrm{~kW} / \mathrm{m} \text { initially; then } 38 \\
\mathrm{~kW} / \mathrm{m}\end{array}$ \\
\hline $\begin{array}{l}\text { Peak internal clad } \\
\text { temperature }\end{array}$ & (not available) \\
\hline $\begin{array}{l}\text { Fluence } \\
\text { (spectrum) }\end{array}$ & $\begin{array}{l}\text { Fluence not available; fast } \\
\text { spectrum }\end{array}$ \\
\hline
\end{tabular}

OTHER FUEL SAMPLE INFORMATION
Each fuel pin was spiral-wrapped with a $1 \mathrm{~mm}$ diameter
spacer wire. Plenum pressure was 1 atm at room
temperature. Above the fuel column were (in series) 17
$\mathrm{~cm}$ of $\mathrm{UO}_{2}$ insulator pellets, $13 \mathrm{~cm}$ of Inconel reflector,
and $29 \mathrm{~cm}$ of spacer tube. The irradiated fue
microstructure was characteristic of high-power-
irradiated fuel, with central void and $\sim 44 \%$ fission gas
retention.

\begin{tabular}{|c|c|}
\hline \multicolumn{2}{|c|}{ TEST CONDITIONS, MEASUREMENTS, \& ANALYSES } \\
\hline $\begin{array}{l}\text { Coolant } \\
\text { environment }\end{array}$ & $\begin{array}{l}\text { Flowing sodium, with } 6 \text {-s flow coastdown to zero } \\
\text { flow beginning at end of preheat }\end{array}$ \\
\hline $\begin{array}{l}\text { Transient } \\
\text { conditions }\end{array}$ & $\begin{array}{l}\text { 2-s preheat at } 52 \mathrm{~kW} / \mathrm{m} \text {, then constant power at } \\
\sim 33 \mathrm{~kW} / \mathrm{m} \text { (average over the pin cluster) for } 23 \\
\mathrm{~s} \text {, then scram, }\end{array}$ \\
\hline $\begin{array}{l}\text { Fuel sample } \\
\text { configuration } \\
\text { in the test }\end{array}$ & $\begin{array}{l}\text { 7-pin bundle of wire-wrapped pins inside a fluted } \\
\text { flow tube surrounded by an inert gas space }\end{array}$ \\
\hline $\begin{array}{l}\text { Test vehicle \& } \\
\text { experiment } \\
\text { system }\end{array}$ & $\begin{array}{l}\text { Mark-IIA integral sodium loop with axial flux- } \\
\text { shaping collars (axial max/avg power }=1.08 \text { ) } \\
\text { test train with argon gas space radially } \\
\text { surrounding the flow tube }\end{array}$ \\
\hline $\begin{array}{l}\text { Measurements } \\
\text { /diagnostics } \\
\text { during test }\end{array}$ & $\begin{array}{l}\text { Temperatures, coolant flow rates, coolant } \\
\text { pressures, fuel motion monitoring by the fast } \\
\text { neutron hodoscope }\end{array}$ \\
\hline $\begin{array}{l}\text { Posttest } \\
\text { coolant flow } \\
\text { condition }\end{array}$ & (not applicable) \\
\hline $\begin{array}{l}\text { Posttest } \\
\text { condition of } \\
\text { test sample(s) }\end{array}$ & $\begin{array}{l}\text { Gross destruction of most of the fuel region. } \\
\text { Accumulations of fuel and steel formed partial } \\
\text { blockages near the top and bottom of the original } \\
\text { fuel region (but the flowtube had been breached) }\end{array}$ \\
\hline $\begin{array}{l}\text { Posttest } \\
\text { measurements } \\
\text { \& information }\end{array}$ & $\begin{array}{l}\text { Neutron and } X \text {-ray radiography, destructive } \\
\text { examination of the remains }\end{array}$ \\
\hline $\begin{array}{l}\text { Posttest } \\
\text { analyses }\end{array}$ & Analysis with SAS2B. \\
\hline
\end{tabular}

\section{KEY SUMMARY INFORMATION ABOUT THE TEST}

In Test L4, coolant boiling apparently began (per indications of channel voiding) $5.4 \mathrm{~s}$ after the start of the 6 -s flow coastdown. Pressure increases about $1.5 \mathrm{~s}$ later suggest cladding failure and release of gas from pin(s). A rapid increase in flowtube temperatures near the top of the fuel region $\sim 3.5 \mathrm{~s}$ later was interpreted as indicating sodium film dryout in the coolant channel. The hodoscope showed the pins to be very flexible and squirming by this time. About $2 \mathrm{~s}$ later, flowtube thermocouples were contacted by materials above the steel melting point, and the hodoscope showed the presence of fuel columns leaning against the loop wall outside the flowtube. During the next $6 \mathrm{~s}$, some fuel moved from above midplane to below midplane, likely in the region outside the flowtube. Two significant pressure-generating events (eructations) occurred in the coolant channel about $2 \mathrm{~s}$ and $6 \mathrm{~s}$ later, during which fuel moved both upward and downward, and then some moved toward the axial midplane. The top third of the fuel did not slump. Little fuel motion happened during the next $3.5 \mathrm{~s}$ until scram occurred. Posttest radiography showed that all of the fuel elements had failed, and masses of fuel (with voids through them) were present at the top and bottoms of the fuel columns, with little fuel in between them. Some fuel had moved to above the original top of the fuel region, extending higher than in test $L 2$ but not as high or as widely distributed as in test L3. At the bottom of the fuel columns, fuel melting was significantly less than had occurred in tests $L 2$ and $L 3$. 


\title{
Summary of TREAT Test L5
}

\author{
Performed 7 November 1974
}

Transient No. 1606

\section{PRE-TEST SAMPLE CHARACTERIZATION}

\begin{tabular}{|l|l|}
\hline \multicolumn{2}{|c|}{ FUEL } \\
\hline Form & He-bonded $\mathrm{MOX}$ \\
\hline Composition & $\begin{array}{l}\mathrm{UO}_{2}-75 \% \mathrm{PuO}_{2}, \text { with the } \mathrm{UO}_{2} \text { being } \\
30 \% \text { enriched }\end{array}$ \\
\hline Geometry & Solid pellet \\
\hline Fissile height & $86.4 \mathrm{~cm}$ \\
\hline $\begin{array}{l}\text { Smear } \\
\text { density }\end{array}$ & $85.0 \%$ of theoretical \\
\hline
\end{tabular}

\begin{tabular}{|l|l|}
\hline \multicolumn{2}{|c|}{ CLADDING } \\
\hline Material & SS $316(20 \% \mathrm{CW})$ \\
\hline OD & $5.84 \mathrm{~mm}$ \\
\hline Thickness & $0.38 \mathrm{~mm}$ \\
\hline
\end{tabular}

\begin{tabular}{|l|l|}
\hline \multicolumn{2}{|l|}{ PRE-IRRADIATION CHARACTERISTICS } \\
\hline BU (peak axial) & 8 at.\% \\
\hline Peak linear power & $40 \mathrm{~kW} / \mathrm{m}$ \\
\hline $\begin{array}{l}\text { Peak internal clad } \\
\text { temperature }\end{array}$ & (not available) \\
\hline $\begin{array}{l}\text { Fluence } \\
\text { (spectrum) }\end{array}$ & $\begin{array}{l}\text { Fluence unavailable; thermal } \\
\text { spectrum }\end{array}$ \\
\hline
\end{tabular}

\section{OTHER FUEL SAMPLE INFORMATION}

Fuel microstructure approximated moderate-powerstructure FFTF fuel. The plenum in each pin was backfilled after irradiation with $\mathrm{Xe}$ and $\mathrm{He}$ at $1 \mathrm{~atm}$. Above and below the fuel column were $13 \mathrm{~mm}$ lengths of $\mathrm{UO}_{2}$ insulator pellets. Above the upper insulator was a 7.6 $\mathrm{cm}$ long Inconel reflector, then a $23 \mathrm{~cm}$ long stainless steel tube.

\begin{tabular}{|l|l|}
\hline \multicolumn{2}{|c|}{ TEST CONDITIONS, MEASUREMENTS, \& ANALYSES } \\
\hline $\begin{array}{l}\text { Coolant } \\
\text { environment }\end{array}$ & $\begin{array}{l}\text { Flowing sodium with an initial rate 40\% of FFTF } \\
\text { nominal, then coasting down for 4.7 s to 15\% of } \\
\text { the initial flow rate starting 4 s into the initial } \\
\text { preheat phase of the transient, }\end{array}$ \\
\hline $\begin{array}{l}\text { Transient } \\
\text { conditions } \\
\text { reactivity step was initiated (when cladding } \\
\text { melting was predicted to have begun), raising } \\
\text { the power on a 0.835 s period and reaching } \\
\text { peak power (nearly six times the preheat power) } \\
\text { 1.6 slater, then scram. }\end{array}$ \\
\hline $\begin{array}{l}\text { Fuel sample } \\
\text { configuration } \\
\text { in the test }\end{array}$ & $\begin{array}{l}\text { 3-pin bunde of wire-wrapped pins inside a 1.47- } \\
\text { mm thick fluted stainless steel flow tube } \\
\text { surrounded by an inert-gas-filled region }\end{array}$ \\
\hline $\begin{array}{l}\text { Test vehicle \& } \\
\text { experiment } \\
\text { system }\end{array}$ & Mark-IIC integral sodium loop \\
\hline $\begin{array}{l}\text { Measurements } \\
\text { diagnostics } \\
\text { during test }\end{array}$ & $\begin{array}{l}\text { Temperatures, coolant flow rates, coolant } \\
\text { pressures, fuel motion monitoring by the 0.5-m } \\
\text { fast neutron hodoscope (over only 60\% of the } \\
\text { test fuel region) }\end{array}$ \\
\hline $\begin{array}{l}\text { Posttest } \\
\text { coolant flow } \\
\text { condition }\end{array}$ & No sodium flow could be achieved posttest. \\
\hline $\begin{array}{l}\text { Posttest } \\
\text { condition of } \\
\text { test sample(s) }\end{array}$ & $\begin{array}{l}\text { Gross fail ure of all pins over nearly the entire } \\
\text { length of the fuel region. }\end{array}$ \\
\hline $\begin{array}{l}\text { Posttest } \\
\text { measurements } \\
\text { \& information }\end{array}$ & $\begin{array}{l}\text { Neutron radiography, gamma-ray scans, macro- } \\
\text { and micro-exainations of the fuel remains }\end{array}$ \\
\hline analyses & Analyses with SAS3A \\
\hline
\end{tabular}

\section{KEY SUMMARY INFORMATION ABOUT THE TEST}

Test L5 was planned to simulate the conditions in moderate-power FFTF subassemblies during a loss-of-flow accident in which fuel slumping in high-power subassemblies causes a power excursion. The actual power burst rose about half as fast and reached only half the peak power than planned, but generated approximately the planned test-fuel energy. There were indications of sodium boiling and flow reversal (voiding) $\sim 0.3 \mathrm{~s}$ before the power burst started. Thermocouple failures indicating steel melting occurred after reactor scram. Most of the fuel motion occurred after scram (when the reactor power had dropped so far that the signal to the hodoscope was too low to be useful). Fuel apparently moved from near the fuel midplane and accumulated at fuel axial locations $x / L=0.11$ to 0.15 and $x / L=0.59$ to 0.66 . Only close to the top and bottom of the original fuel region was the original geometry maintained. Nearly complete blockages of solidified steel filling the flow channel around the fuel elements formed between 7 and $33 \mathrm{~mm}$ above the top of the original fuel column (at the bottom of the reflector rod). A blockage also formed between 8 and $25 \mathrm{~mm}$ above the bottom of the fuel region and consisted of steel that had filled almost the entire cross section of the flow tube including most of the area originally occupied by fuel. The cladding tubes were intact below the lower blockage but completely destroyed above the blockage. Just below the upper blockage was an accumulation of many fuel chunks of size 0.5 to $3 \mathrm{~mm}$, mixed with steel. Chunks of fuel were present everywhere above and below the region $x / L=0.2$ to 0.8 . It appeared that most of the molten cladding moved either upward to form the upper blockage or laterally to mix with melted parts of the flowtube before draining. All disrupted fuel remained between the upper and lower cladding blockages and little was found outside the flowtube. 


\section{Summary of TREAT Test L6}

Performed 18 October 1978

Transient No. 2068

\section{PRE-TEST SAMPLE CHARACTERIZATION}

\begin{tabular}{|l|l|}
\hline \multicolumn{2}{|c|}{ FUEL } \\
\hline Form & He-bonded MOX \\
\hline Composition & $\mathrm{UO}_{2}-75 \% \mathrm{PuO}_{2}$ \\
\hline Geometry & Solid pellet with dished ends \\
\hline Fissile height & $86.4 \mathrm{~cm}$ \\
\hline $\begin{array}{l}\text { Smear } \\
\text { density }\end{array}$ & $85.5 \%$ of theoretical \\
\hline
\end{tabular}

\begin{tabular}{|l|l|}
\hline \multicolumn{2}{|c|}{ CLADDING } \\
\hline Material & SS $316(20 \% \mathrm{CW})$ \\
\hline OD & $5.84 \mathrm{~mm}$ \\
\hline Thickness & $0.38 \mathrm{~mm}$ \\
\hline
\end{tabular}

\begin{tabular}{|l|l|}
\hline \multicolumn{2}{|l|}{ PRE-IRRADIATION CHARACTERISTICS } \\
\hline BU (peak axial) & 3 at.\% \\
\hline Peak linear power & $41 \mathrm{~kW} / \mathrm{m}$ \\
\hline $\begin{array}{l}\text { Peak internal clad } \\
\text { temperature }\end{array}$ & (not available) \\
\hline $\begin{array}{l}\text { Fluence } \\
\text { (spectrum) }\end{array}$ & $\begin{array}{l}\text { Fluence unavail able; thermal } \\
\text { spectrum }\end{array}$ \\
\hline
\end{tabular}

\section{OTHER FUEL SAMPLE INFORMATION}

Fuel microstructure approximated moderate-powerstructure FFTF fuel. The plenum in each pin was backfilled after irradiation with $\mathrm{Xe}$ and $\mathrm{He}$ at $1 \mathrm{~atm}$. Above and below the fuel column were $13 \mathrm{~mm}$ lengths of $\mathrm{UO}_{2}$ insulator pellets. Above the upper insulator was a 7.6 $\mathrm{cm}$ long Inconel reflector, then a $23 \mathrm{~cm}$ long stainless steel tube.

\begin{tabular}{|l|l|}
\hline \multicolumn{2}{|c|}{ TEST CONDITIONS, MEASUREMENTS, \& ANALYSES } \\
\hline $\begin{array}{l}\text { Coolant } \\
\text { environment }\end{array}$ & $\begin{array}{l}\text { Flowing sodium with an initial rate 40\% of FFTF } \\
\text { nominal, then coasting down starting } 4 \mathrm{~s} \text { into the } \\
\text { initial preheat phase of the transient, }\end{array}$ \\
\hline $\begin{array}{l}\text { Transient } \\
\text { conditions }\end{array}$ & $\begin{array}{l}\text { Constant power at } ~ 36 \mathrm{~kW} / \mathrm{m} \text { for } 9 \mathrm{~s} \text {; then rapid } \\
\text { power increase to } 10 \text { times nominal. Scram rod } \\
\text { motion began } 200 \mathrm{~ms} \text { after peak power (allowing } \\
\text { hodoscope viewing during the expected time of } \\
\text { fuel motion). }\end{array}$ \\
\hline $\begin{array}{l}\text { Fuel sample } \\
\text { configuration } \\
\text { in the test }\end{array}$ & $\begin{array}{l}\text { 3-pin bundle of wire-wrapped pins inside a 1.47- } \\
\text { mm thick fluted stainless steel flow tube } \\
\text { surrounded by an inert-gas-filled region }\end{array}$ \\
\hline $\begin{array}{l}\text { Test vehicle \& } \\
\text { experiment } \\
\text { system }\end{array}$ & Mark-IIC integral sodium loop \\
\hline $\begin{array}{l}\text { Measurements } \\
\text { /diagnostics } \\
\text { during test }\end{array}$ & $\begin{array}{l}\text { Temperatures, coolant flow rates, codant } \\
\text { pressures, fuel motion monitoring by the 1.2-m } \\
\text { fast neutron hodoscope }\end{array}$ \\
\hline $\begin{array}{l}\text { Posttest } \\
\text { coolant flow } \\
\text { condition }\end{array}$ & $\begin{array}{l}\text { Posttest coolant flow capability information was } \\
\text { unavailable. }\end{array}$ \\
\hline $\begin{array}{l}\text { Posttest } \\
\text { condition of } \\
\text { test sample(s) }\end{array}$ & Gross disruption of the original fuel region. \\
\hline $\begin{array}{l}\text { Posttest } \\
\text { measurements } \\
\text { \& information }\end{array}$ & Neutron radiography \\
\hline $\begin{array}{l}\text { Posttest } \\
\text { analyses }\end{array}$ & $\begin{array}{l}\text { Analyses of fuel redistribution in terms of } \\
\text { equivalent worth changes. }\end{array}$ \\
\hline
\end{tabular}

\section{KEY SUMMARY INFORMATION ABOUT THE TEST}

Test L6 duplicated the sequence of test L5 (flow coastdown, coolant voiding, cladding motion, and then fuel motion) until the initiation of the power increase, and generated the same amount of test-fuel energy up to peak power. When tests $L 5$, L6, and L7 were being planned, it was considered possible to terminate an LOF accident by early fuel dispersal driven mainly by release of entrained fission gases, so a test objective was to avoid generating significant fuel vapor pressures. It was calculated that the maximum fuel temperatures during the test did not exceed $4000^{\circ} \mathrm{C}$, and thus no significant fuel vapor pressure was generated. The change in axial distribution of fuel during the test, as determined by the hodoscope, can be described in terms of the corresponding changes in fuel worth if those fuel motions were to occur in a fast power reactor (using the central subassemblies of CRBR as an example). In L6, the fuel worth declined by $\sim 3 \%$ during the 200 ms between peak power and scram. No pressure pulses were observed during the test. 


\section{Summary of TREAT Test L7}

Performed 10 September 1977

Transient No. 1932

\section{PRE-TEST SAMPLE CHARACTERIZATION}

\begin{tabular}{|l|l|}
\hline \multicolumn{2}{|c|}{ FUEL } \\
\hline Form & He-bonded $\mathrm{MOX}$ \\
\hline Composition & $\mathrm{UO}_{2}-75 \% \mathrm{PuO}_{2}$ \\
\hline Geometry & Solid pellet with dished ends \\
\hline Fissile height & $86.4 \mathrm{~cm}$ \\
\hline $\begin{array}{l}\text { Smear } \\
\text { density }\end{array}$ & $85.5 \%$ of theoretical \\
\hline
\end{tabular}

\begin{tabular}{|l|l|}
\hline \multicolumn{2}{|c|}{ CLADDING } \\
\hline Material & SS $316(20 \% \mathrm{CW})$ \\
\hline OD & $5.84 \mathrm{~mm}$ \\
\hline Thickness & $0.38 \mathrm{~mm}$ \\
\hline
\end{tabular}

\begin{tabular}{|l|l|}
\hline \multicolumn{2}{|l|}{ PRE-IRRADIATION CHARACTERISTICS } \\
\hline BU (peak axial) & 3 at.\% \\
\hline Peak linear power & $41 \mathrm{~kW} / \mathrm{m}$ ("nominal") \\
\hline $\begin{array}{l}\text { Peak internal clad } \\
\text { temperature }\end{array}$ & (not available) \\
\hline $\begin{array}{l}\text { Fluence } \\
\text { (spectrum) }\end{array}$ & $\begin{array}{l}\text { Fluence unavailable; thermal } \\
\text { spectrum }\end{array}$ \\
\hline
\end{tabular}

\section{OTHER FUEL SAMPLE INFORMATION}

Fuel microstructure was like moderate-power-structure FFTF fuel. Plenum back-filled after irradiation with $\mathrm{Xe}$ and $\mathrm{He}$ at $1 \mathrm{~atm}$. Above and below the fuel column were $13 \mathrm{~mm}$ lengths of $\mathrm{UO}_{2}$ insulator pellets. Above the upper insulator was a $7.6 \mathrm{~cm}$ long Inconel reflector, then a 23 $\mathrm{cm}$ long stainless steel tube.

\begin{tabular}{|l|l|}
\hline \multicolumn{2}{|c|}{ TEST CONDITIONS, MEASUREMENTS, \& ANALYSES } \\
\hline $\begin{array}{l}\text { Coolant } \\
\text { environment }\end{array}$ & $\begin{array}{l}\text { Flowing sodium with an initial rate 40\% of FFTF } \\
\text { nominal, then coasting down starting 4 s into the } \\
\text { initial preheat phase of the transient, }\end{array}$ \\
\hline $\begin{array}{l}\text { Transient } \\
\text { conditions }\end{array}$ & $\begin{array}{l}\sim 36 \mathrm{~kW} / \mathrm{m} \text { for } 9 \mathrm{~s} \text {; then rapid power increase to } \\
23 \text { times nominal. Scram rod motion began } \\
\text { after peak power to allow adequate hodoscope } \\
\text { signal level during the expected time of fuel } \\
\text { motion. }\end{array}$ \\
\hline $\begin{array}{l}\text { Fuel sample } \\
\text { configuration } \\
\text { in the test }\end{array}$ & $\begin{array}{l}\text { 3-pin bunde of wire-wrapped pins, inside a 1.47- } \\
\text { mm-thick 16-mm-OD fluted stainless sted flow } \\
\text { tube, surrounded by an inert-gas-filled region, } \\
\text { bounded by a 0.9-mm-thick 25-mm-OD SS } \\
\text { "inner tube." }\end{array}$ \\
\hline $\begin{array}{l}\text { Test vehicle \& } \\
\text { experiment } \\
\text { system }\end{array}$ & Mark-IIC integral sodium loop \\
\hline $\begin{array}{l}\text { Measurements } \\
\text { /diagnostics } \\
\text { during test }\end{array}$ & $\begin{array}{l}\text { Temperatures, coolant flow rates, coolant } \\
\text { pressures, fuel motion monitoring by the 1.2-m } \\
\text { fast neutron hodoscope }\end{array}$ \\
\hline $\begin{array}{l}\text { Posttest } \\
\text { coolant flow } \\
\text { condition }\end{array}$ & Information not available \\
\hline $\begin{array}{l}\text { Posttest } \\
\text { condition of } \\
\text { test sample(s) }\end{array}$ & Great disruption of the original fuel region. \\
\hline $\begin{array}{l}\text { Posttest } \\
\text { measurements } \\
\text { \& information }\end{array}$ & Neutron radiography \\
\hline $\begin{array}{l}\text { Posttest } \\
\text { analyses }\end{array}$ & $\begin{array}{l}\text { Analyses of fuel redistribution in terms of } \\
\text { SAS3D (with PRIMAR-3) }\end{array}$ \\
\hline
\end{tabular}

\section{KEY SUMMARY INFORMATION ABOUT THE TEST}

Test L7 (like Test L6) duplicated the sequence of tests L5 and L6 (flow coastdown, coolant voiding, cladding motion, and then fuel motion) until the initiation of the power increase, and generate the same amount of test-fuel energy up to peak power as in L5. When tests L5, L6, and $\mathrm{L} 7$ were being planned, it was considered possible to terminate an LOF accident by early fuel dispersal driven mainly by release of entrained fission gases, so a test objective was to avoid generating significant fuel vapor pressures. It was calculated posttest that the maximum fuel temperature exceeded $4000^{\circ} \mathrm{C}$ (above which significant fuel vapor pressure would be generated) beginning about half way between peak power and scram. Instrumentation indicated that sodium boiling started about $0.3 \mathrm{~s}$ before the power burst began, and that voiding continued slowly for about $0.6 \mathrm{~s}$. At that point (when the power was 17 times nominal, $0.13 \mathrm{~s}$ before peak power), sodium was rapidly ejected from the fuel region, with no pressure pulses being detected. The important fuel movements occurred during the 200$\mathrm{ms}$ period between peak power and scram. At the end of the test, most of the fuel from the bottom $60 \%$ of the fuel region had relocated to the top $40 \%$ of the fuel region and slightly higher, and much fuel had moved outside the flowtube wall and collected in a large-diameter mass near the top of the fuel region. Only a little fuel moved axially more than $\sim 2 \mathrm{~cm}$ above the original top of the fuel region, and little had moved beyond the original bottom of the fuel region. The change in axial distribution of fuel during the test, as determined by the hodoscope, can be described in terms of the corresponding changes in fuel worth if those fuel motions were to occur in a fast power reactor (using the central subassemblies of CRBR as an example). In L7, the fuel worth declined by $\sim 10 \%$, at a nearly-constant rate of $\sim 60 \mathrm{~d} / \mathrm{s}$ per dollar from the onset of fuel motion to scram. However, at the time the peak fuel temperature was calculated to have reached $4000^{\circ} \mathrm{C}$ (calculated), the equivalent fuel worth had decreased by only $\sim 4 \phi / s$ per dollar relative to intact geometry. 


\section{Summary of TREAT Test L8 \\ Performed 9 May 1978 \\ Transient No. 2007}

\section{PRE-TEST SAMPLE CHARACTERIZATION}

\begin{tabular}{|l|l|}
\hline \multicolumn{2}{|c|}{ FUEL } \\
\hline Form & He-bonded MOX \\
\hline Composition & $\mathrm{UO}_{2}-75 \% \mathrm{PuO}_{2}$ (U was 30\% enr.) \\
\hline Geometry & Solid pellet with dished ends \\
\hline Fissile height & $86.4 \mathrm{~cm}$ \\
\hline $\begin{array}{l}\text { Smear } \\
\text { density }\end{array}$ & $85.5 \%$ of theoretical \\
\hline
\end{tabular}

\begin{tabular}{|l|l|}
\hline \multicolumn{2}{|c|}{ CLADDING } \\
\hline Material & SS $316(20 \% \mathrm{CW})$ \\
\hline OD & $5.84 \mathrm{~mm}$ \\
\hline Thickness & $0.38 \mathrm{~mm}$ \\
\hline
\end{tabular}

\begin{tabular}{|l|l|}
\hline \multicolumn{2}{|l|}{ PRE-IRRADIATION CHARACTERISTICS } \\
\hline BU (peak axial) & 3 at.\% \\
\hline Peak linear power & $20.3 \mathrm{~kW} / \mathrm{m}$ ("nominal") \\
\hline $\begin{array}{l}\text { Peak internal clad } \\
\text { temperature }\end{array}$ & (not available) \\
\hline $\begin{array}{l}\text { Fluence } \\
\text { (spectrum) }\end{array}$ & $\begin{array}{l}\text { Fluence unavail able; thermal } \\
\text { spectrum }\end{array}$ \\
\hline
\end{tabular}

\section{OTHER FUEL SAMPLE INFORMATION}

Irradiated fuel was unrestructured, to represent lowpower FFTF fuel. Plenum back-filled after irradiation with $\mathrm{Xe}$ and $\mathrm{He}$ at $1 \mathrm{~atm}$. Above and below the fuel column were 13 mm lengths of $\mathrm{UO}_{2}$ insulator pellets. Above the upper insulator was a $7.6 \mathrm{~cm}$ long Inconel reflector, then a $23 \mathrm{~cm}$ long stainless steel tube. About $10 \%$ fission gas released from pre-tested fuel.

\begin{tabular}{|c|c|}
\hline \multicolumn{2}{|c|}{ TEST CONDITIONS, MEASUREMENTS, \& ANALYSES } \\
\hline $\begin{array}{l}\text { Coolant } \\
\text { environment }\end{array}$ & $\begin{array}{l}\text { Flowing sodium with an initial rate of } \sim 180 \\
\mathrm{~cm} 3 / \mathrm{s} \text {, then coasting down at } \sim 20 \mathrm{~cm} 3 / \mathrm{s} \text { per } \\
\text { second starting } 0.5 \text { s before the beginning of the } \\
\text { power hold }\end{array}$ \\
\hline $\begin{array}{l}\text { Transient } \\
\text { conditions }\end{array}$ & $\begin{array}{l}\text { 2.4-s hold at } 3.9 \text { times nominal power, then } \\
\text { power burst of } 255 \text { ms full-width-at-half- } \\
\text { maximum that peaked at } 75 \text { times nominal }\end{array}$ \\
\hline $\begin{array}{l}\text { Fuel sample } \\
\text { configuration } \\
\text { in the test }\end{array}$ & $\begin{array}{l}\text { 3-pin bunde of wire-wrapped pins, inside a } 1.47 \text { - } \\
\text { mm-thick } 16-\mathrm{mm}-\mathrm{OD} \text { fluted stainless steel flow } \\
\text { tube, surrounded by an inert-gas-filled region, } \\
\text { bounded by a } 0.9-\mathrm{mm} \text {-thick } 25 \text {-mm-OD SS } \\
\text { "intermediate tube," surrounded by a 0.9-mm- } \\
\text { thick } 31 \text {-mm-OD "outer tube." }\end{array}$ \\
\hline $\begin{array}{l}\text { Test vehicle \& } \\
\text { experiment } \\
\text { system }\end{array}$ & Mark-IICB integral sodium loop \\
\hline $\begin{array}{l}\text { Measurements } \\
\text { /diagnostics } \\
\text { during test }\end{array}$ & $\begin{array}{l}\text { Temperatures, coolant flow rates, coolant } \\
\text { pressures, fuel motion monitoring by the } 1.2-\mathrm{m} \\
\text { fast neutron hodoscope }\end{array}$ \\
\hline $\begin{array}{l}\text { Posttest } \\
\text { coolant flow } \\
\text { condition }\end{array}$ & Posttest coolant flow capability is unavailable. \\
\hline $\begin{array}{l}\text { Posttest } \\
\text { condition of } \\
\text { test sample(s) }\end{array}$ & Gross disruption of the original fuel region. \\
\hline $\begin{array}{l}\text { Posttest } \\
\text { measurements } \\
\text { \& information }\end{array}$ & $\begin{array}{l}\text { Posttest destructive examination of the fuel } \\
\text { remains. }\end{array}$ \\
\hline $\begin{array}{l}\text { Posttest } \\
\text { analyses }\end{array}$ & $\begin{array}{l}\text { Analyses of fuel redistribution data in terms of } \\
\text { equivalent worth change. } \\
\text { Analyses with SAS3D, LEVITATE, and PLUTO2 }\end{array}$ \\
\hline
\end{tabular}

\section{KEY SUMMARY INFORMATION ABOUT THE TEST}

Test $L 8$ achieved its objectives of causing initial failure of a low-power-structure pin to occur at a power level at least 30 times nominal, with failure into almost-boiling flowing sodium. The total test-fuel energy generated was similar to that in tests $L 5, L 6$, and L7. Sharp pressure pulses and flow anomalies occurred as the power was rising past 75 times nominal power, indicating cladding failure. Fuel motion that occurred within $0.1 \mathrm{~s}$ after cladding failure amounted to an equivalent reactivity worth loss of $15-20 \%$ (if described in terms of the corresponding changes in fuel worth if those fuel motions were to occur in a fast power reactor [using the central subassemblies of CRBR as an example]). The worth value did not change during the next $0.15 \mathrm{~s}$ as the power level began dropping from its peak. Much of the flowtube melted, except at the top and bottom end of the original fuel region. Melting of the "intermediate tube" occurred mostly above the fuel midplane. The fuel posttest axial distribution was as follows: $\sim 10 \%$ was below the original fuel region (some fuel as far as $21 \mathrm{~cm}$ below); $20 \%$ within the bottom half of the original region (but practically no fuel at $\mathrm{x} / \mathrm{L}=0.37-0.45$ ); $\sim 60 \%$ in the upper half of the original fuel region; and $\sim 10 \%$ above the original fuel region (some extending as far as $40 \mathrm{~cm}$ above). Over the range $x / L=$ 0.57 to 0.73 , the fuel was limited radially by the "outer tube." From $x / L=0.85$ to 1.00 it was limited radially by the "intermediate tube." Generally, the fuel was porous and brittle. Some fuel that moved downward had to flow within the annular space between the flowtube and the intermediate tube because spongy was fuel blocking the interior of the flowtube. There was no indication that the melted cladding moved upward, and there was no accumulation of melted steel in the upper part of the original fuel region or above. Much of the fuel contained finely-dispersed steel. 


\section{Appendix I: One-page Summaries of PINEX-series Tests}

One-page summaries of each of the PINEX-series tests are included here. These summaries can also be found in TREXR [6]. 


\section{Summary of TREAT Test PINEX-2 \\ Performed 16 January 1978 \\ Transient No. 1986}

\section{PRE-TEST SAMPLE CHARACTERIZATION}

\begin{tabular}{|l|l|}
\hline \multicolumn{2}{|c|}{ FUEL } \\
\hline Form & He-bonded MOX \\
\hline Composition & $\begin{array}{l}25 \text { wt \% PuO2, 75 wt \% UO2 } \\
\text { (natural U) }\end{array}$ \\
\hline Geometry & $\begin{array}{l}\text { Annular pellets } \\
(0.81 \mathrm{~mm} \mathrm{ID})\end{array}$ \\
\hline Fissile height & $86.4 \mathrm{~cm}$ \\
\hline $\begin{array}{l}\text { Smear } \\
\text { density }\end{array}$ & $86.1 \%$ of theoretical density \\
\hline
\end{tabular}

\begin{tabular}{|l|l|}
\hline \multicolumn{2}{|c|}{ CLADDING } \\
\hline Material & $316 \mathrm{SS}, 20 \% \mathrm{CW}$ \\
\hline OD & $0.584 \mathrm{~cm}$ \\
\hline Thickness & $0.038 \mathrm{~cm}$ \\
\hline
\end{tabular}

\begin{tabular}{|l|l|}
\hline \multicolumn{2}{|l|}{ PRE-IRRADIATION CHARACTERISTICS } \\
\hline BU (peak axial) & $\begin{array}{l}19.5 \mathrm{GWd} \\
\mathrm{HM} \text { ) }\end{array}$ \\
\hline Peak linear power & $41 \mathrm{~kW} / \mathrm{m}$ at\% of \\
\hline $\begin{array}{l}\text { Peak internal clad } \\
\text { temperature }\end{array}$ & (unknown) \\
\hline $\begin{array}{l}\text { Fluence } \\
\text { (spectrum) }\end{array}$ & $\begin{array}{l}\text { Fluence is unavailable; } \\
\text { thermal spectrum (GETR) }\end{array}$ \\
\hline
\end{tabular}

\section{OTHER FUEL SAMPLE INFORMATION}

Fuel mass $=165 \mathrm{~g}$. Upper $\mathrm{UO}_{2}$ insulator col umn $4.83 \mathrm{~mm} \mathrm{OD}, 1.78 \mathrm{~mm} \mathrm{ID}, 12.7 \mathrm{~mm}$ high. Reflector $I D=1.70 \mathrm{~mm}$, length $4.93 \mathrm{~mm}$. Initial plenum pressure of 1.6 MPa. The pre-irradiation caused closure of top 15 $\mathrm{cm}$ of the central void in the fuel.

\begin{tabular}{|c|c|}
\hline \multicolumn{2}{|c|}{ TEST CONDITIONS, MEASUREMENTS, \& ANALYSES } \\
\hline $\begin{array}{l}\text { Coolant } \\
\text { environment }\end{array}$ & Static NaK \\
\hline $\begin{array}{l}\text { Transient } \\
\text { conditions }\end{array}$ & $\begin{array}{l}\text { Initial power flattop, then a power ramp to } \\
\text { simulate a } 5 \$ / \mathrm{sec} \text { overpower transient. Power } \\
\text { burst full-width at half maximum was } \sim 0.2 \mathrm{~s} \text {. }\end{array}$ \\
\hline $\begin{array}{l}\text { Fuel sample } \\
\text { configuration } \\
\text { in the test }\end{array}$ & Single pin \\
\hline $\begin{array}{l}\text { Test vehicle \& } \\
\text { experiment } \\
\text { system }\end{array}$ & $\begin{array}{l}\text { GETR/TREAT Mark II type capsule, with axial } \\
\text { power shaping such that the top of the fuel } \\
\text { received about } 40 \% \text { power compared to the fuel } \\
\text { midplane }\end{array}$ \\
\hline $\begin{array}{l}\text { Measurements } \\
\text { /diagnostics } \\
\text { during test }\end{array}$ & $\begin{array}{l}\text { PINEX pinhole imaging system; fuel motion } \\
\text { (hodoscope), temperatures near fuel pin }\end{array}$ \\
\hline $\begin{array}{l}\text { Posttest } \\
\text { coolant flow } \\
\text { condition }\end{array}$ & (not applicable) \\
\hline $\begin{array}{l}\text { Posttest } \\
\text { condition of } \\
\text { test sample(s) }\end{array}$ & $\begin{array}{l}\text { No cladding failure. } 10 \mathrm{~g} \text { of molten fuel ejected } \\
\text { upward into reflector region and fission gas } \\
\text { plenum. Maximum cladding strain of } 1.0 \% \text {. Fuel } \\
\text { melting occurred over relative fuel height } 0.15 \text { to } \\
0.70 \text {. }\end{array}$ \\
\hline $\begin{array}{l}\text { Posttest } \\
\text { measurements } \\
\text { \& information }\end{array}$ & $\begin{array}{l}\text { Neutron radiography, } x \text {-radiography, } \\
\text { ceramographic microstructural examination }\end{array}$ \\
\hline $\begin{array}{l}\text { Posttest } \\
\text { analyses }\end{array}$ & $\begin{array}{l}\text { Code comparison among the ORIGEN, TEMP, } \\
\text { LAFM, HOTPIM, FUMO-T, FRAX-2, and PINEX- } \\
\text { AR codes. }\end{array}$ \\
\hline
\end{tabular}

\section{KEY SUMMARY INFORMATION ABOUT THE TEST}

PINEX-2 was conducted to demonstrate the mitigation effects of internal fuel motion in a hypothetical transient overpower accident and to verify the PINEX pinhole diagnostic system concept An annular design was employed to promote fuel relocation to the plenum. The power transient was designed to provide sufficient fuel melting and fission gas release to make fuel motion likely and to be high enough for fuel detection by the PINEX camera system. Scram was programmed to occur at the time of initial fuel melting, to reflect the possible negative reactivity effect that axial fuel extrusion would have in a power reactor. The pin survived to $\sim 27$ times nominal power. The PINEX system capability was demonstrated as intended. The hodoscope detected six fuel-motion events, including three after scram. Posttest examination of the pin showed once-molten fuel filled the passage through the reflector, more than half the available volume in the pin plenum, and the fuel column central void above and below the region of gross melting. 


\section{Summary of TREAT Test PINEX-3 \\ Performed 27 February 1980 \\ Transient No. 2196}

\begin{tabular}{l}
\hline \multicolumn{2}{|l|}{ PRE-TEST SAMPLE CHARACTERIZATION } \\
\begin{tabular}{|l|l|}
\hline \multicolumn{2}{|c|}{ FUEL } \\
\hline Form & He-bonded MOX \\
\hline Composition & $\begin{array}{l}25 \text { wt } \% \text { PuO2, 75 w \% UO2 } \\
\text { (natural U) }\end{array}$ \\
\hline Geometry & $\begin{array}{l}\text { Annular pellets } \\
(0.81 \text { mm ID) }\end{array}$ \\
\hline Fissile height & $86.36 \mathrm{~cm}$ \\
\hline $\begin{array}{l}\text { Smear } \\
\text { density }\end{array}$ & $86.1 \%$ of theoretical density \\
\hline
\end{tabular}
\end{tabular}

\begin{tabular}{|l|l|}
\hline \multicolumn{2}{|c|}{ CLADDING } \\
\hline Material & $316 \mathrm{SS}, 20 \% \mathrm{CW}$ \\
\hline OD & $0.584 \mathrm{~cm}$ \\
\hline Thickness & $0.038 \mathrm{~cm}$ \\
\hline
\end{tabular}

\begin{tabular}{|l|l|}
\hline \multicolumn{2}{|l|}{ PRE-IRRADIATION CHARACTERISTICS } \\
\hline BU (peak axial) & $\begin{array}{l}19.5 \mathrm{GWd} \\
\mathrm{HM} \text { ) }\end{array}$ \\
\hline Peak linear power & $41 \mathrm{~kW} / \mathrm{m}$ at\% of \\
\hline $\begin{array}{l}\text { Peak internal clad } \\
\text { temperature }\end{array}$ & (unknown) \\
\hline $\begin{array}{l}\text { Fluence } \\
\text { (spectrum) }\end{array}$ & $\begin{array}{l}\text { Fluence is unavailable; } \\
\text { thermal spectrum (GETR) }\end{array}$ \\
\hline
\end{tabular}

\section{OTHER FUEL SAMPLE INFORMATION}

Fuel mass $=165 \mathrm{~g}$. Upper $\mathrm{UO}_{2}$ insulator column $4.83 \mathrm{~mm} \mathrm{OD}, 1.78 \mathrm{~mm} \mathrm{ID}, 12.7 \mathrm{~mm}$ high. Reflector $\mathrm{ID}=1.70 \mathrm{~mm}$, length $4.93 \mathrm{~mm}$. Initial plenum pressure of 1.6 $\mathrm{MPa}$. The pre-irradiation caused closure of top 15 $\mathrm{cm}$ of the central void in the fuel.

\begin{tabular}{|c|c|}
\hline \multicolumn{2}{|c|}{ TEST CONDITIONS, MEASUREMENTS, \& ANALYSES } \\
\hline $\begin{array}{l}\text { Coolant } \\
\text { environment }\end{array}$ & Static NaK \\
\hline $\begin{array}{l}\text { Transient } \\
\text { conditions }\end{array}$ & $\begin{array}{l}\text { Initial low-level steady-state at } 40 \mathrm{~kW} / \mathrm{m} \text { for } 5.5-\mathrm{s} \\
\text { duration followed by a } 50 \mathrm{~s} / \mathrm{s} \text { TOP to maximum } \\
\text { power of } 300 \mathrm{~kW} / \mathrm{m}\end{array}$ \\
\hline $\begin{array}{l}\text { Fuel sample } \\
\text { configuration } \\
\text { in the test }\end{array}$ & Single pin \\
\hline $\begin{array}{l}\text { Test vehicle \& } \\
\text { experiment } \\
\text { system }\end{array}$ & $\begin{array}{l}\text { Nickel heat sink static capsule in containment } \\
\text { vessel, } B_{6} \text { Si axial flux shaping collars }\end{array}$ \\
\hline $\begin{array}{l}\text { Measurements } \\
\text { /diagnostics } \\
\text { during test }\end{array}$ & $\begin{array}{l}\text { Fuel motion (hodoscope), photography (pinhole } \\
\text { imaging system), thermocouples }\end{array}$ \\
\hline $\begin{array}{l}\text { Posttest } \\
\text { coolant flow } \\
\text { condition }\end{array}$ & (not applicable) \\
\hline $\begin{array}{l}\text { Posttest } \\
\text { condition of } \\
\text { test sample(s) }\end{array}$ & $\begin{array}{l}\text { Massive cladding failure, no significant } \\
\text { redistribution of fuel to outside of pin, only the } \\
\text { top } 18 \% \text { of the fuel and cladding in the initial fuel } \\
\text { region remained intact }\end{array}$ \\
\hline $\begin{array}{l}\text { Posttest } \\
\text { measurements } \\
\text { \& information }\end{array}$ & $\begin{array}{l}\text { X-radiography, neutron radiography, destructive } \\
\text { examination, microstructure analysis }\end{array}$ \\
\hline $\begin{array}{l}\text { Posttest } \\
\text { analyses }\end{array}$ & Analyses with TEMP, LAFM, FUMO-E codes. \\
\hline
\end{tabular}

\section{KEY SUMMARY INFORMATION ABOUT THE TEST}

PINEX-3 was conducted to demonstrate the mitigation effects of internal fuel motion in a hypothetical transient overpower accident and to verify the PINEX pinhole diagnostic system concept. An annular design was employed for the fuel, reflectors, and insulators to promote fuel relocation to the fission gas plenum. The power transient was designed to provide sufficient fuel melting and fission gas release to make fuel motion likely and to be high enough for fuel detection by the PINEX camera system. Scram was programmed to occur at the time of initial fuel melting, to reflect the possible negative reactivity effect that axial fuel extrusion would have in a power reactor. Cladding failure occurred without ejection of large quantities of molten fuel into the fission gas plenum. Fuel relocated from below the midplane to above the midplane, followed by gradual net downward movement of fuel. Posttest examination showed some fuel in the upper reflector region, a small plug of fuel in the lower insulator pellet, and a likely blockage in the axial hole in the upper fuel region. Results from the PINEX system were compared with those from the hodoscope. 


\section{Appendix J: One-page Summaries of RFT-series Tests}

One-page summaries of each of the RFT-series tests are included here. These summaries can also be found in TREXR [6]. 


\title{
Summary of TREAT Test RFT-L1
}

\author{
Performed August 1982
}

\begin{tabular}{l}
\hline \multicolumn{2}{|c|}{ PRE-TEST SAMPLE CHARACTERIZATION } \\
\begin{tabular}{|l|l|}
\hline \multicolumn{2}{|c|}{ FUEL } \\
\hline Form & He-bonded MOX \\
\hline Composition & $\begin{array}{l}\text { Pin 1: } \mathrm{UO}_{2}-22 \% \mathrm{PuO}_{2} \\
\text { Pin 2: } \mathrm{UO}_{2}-22 \% \mathrm{PuO}_{2} \\
\text { Pin 3: } \mathrm{UO}_{2}-27 \% \mathrm{PuO}_{2} \\
\left.\text { (Natural } \mathrm{UO}_{2}, \mathrm{O} / \mathrm{M}=1.96\right)\end{array}$ \\
\hline Geometry & Solid pellet \\
\hline Fissile height & $\begin{array}{l}\text { Pin 1: } 91.2 \mathrm{~cm} \\
\text { Pin 2: } 91.4 \mathrm{~cm} \\
\text { Pin 3: } 91.8 \mathrm{~cm}\end{array}$ \\
\hline $\begin{array}{l}\text { Smear } \\
\text { density }\end{array}$ & $\sim 85 \%$ of theoretical \\
\hline
\end{tabular}
\end{tabular}

\begin{tabular}{|l|l|}
\hline \multicolumn{2}{|c|}{ TEST CONDITIONS, MEASUREMENTS, \& ANALYSES } \\
\hline $\begin{array}{l}\text { Coolant } \\
\text { environment }\end{array}$ & Flowing sodium \\
\hline $\begin{array}{l}\text { Transient } \\
\text { conditions }\end{array}$ & $\begin{array}{l}\text { Short }(\sim 6 \mathrm{~s}) \text { preheat at slightly more than } \\
\text { nominal power, then FFTF 50 c/s overpower } \\
\text { ramp reaching about 5 times nominal pin power } \\
\text { in 2.4 s, then scram. } \\
\text { Pin 1 power was 5\% higher than Pin 2 power } \\
\text { and 4\%lower than Pin 3 power. }\end{array}$ \\
\hline $\begin{array}{l}\text { Fuel sample } \\
\text { configuration } \\
\text { in the test }\end{array}$ & Three pins in separate flow tubes \\
\hline $\begin{array}{l}\text { Test vehicle \& } \\
\text { experiment } \\
\text { system }\end{array}$ & $\begin{array}{l}\text { Mark-IIIA sodium loop, with axial flux-shaping } \\
\text { collars }\end{array}$ \\
\hline $\begin{array}{l}\text { Measurements } \\
\text { /diagnostics } \\
\text { during test }\end{array}$ & Thermocouples, flowmeters \\
\hline $\begin{array}{l}\text { Posttest } \\
\text { coolant flow } \\
\text { condition }\end{array}$ & (not available) \\
\hline $\begin{array}{l}\text { Posttest } \\
\text { condition of } \\
\text { test sample(s) }\end{array}$ & Cladding of only Pin 3 failed (at x/L = 0.8). \\
\hline $\begin{array}{l}\text { Posttest } \\
\text { measurements } \\
\text { \& information }\end{array}$ & \begin{tabular}{l} 
Macro-examination, neutron radiography, \\
gamma scanning, fission gas sampling, \\
\hline $\begin{array}{l}\text { Posttest } \\
\text { analyses }\end{array}$
\end{tabular} \\
\hline
\end{tabular}

\section{OTHER FUEL SAMPLE INFORMATION}

FFTF driver fuel pins; $145 \mathrm{~mm}$ long Inconel reflector above upper insulator pellets

\section{KEY SUMMARY INFORMATION ABOUT THE TEST}

Test RFT-L1 was conducted to investigate the effects of a $50 \% / s$ unprotected overpower transient on very low burnup FFTF driver fuel pins of different irradiation power levels. The test conditions were selected to cause fuel melting and cladding strain but no cladding failure. The failure of Pin 3 may have been due to the fact that its power level was lower than that of the other two pins, and it had been irradiated at a lower temperature than the other pins. A hole was found in the flowtube of Pin 3 near the elevation where Pin 3 failed. All pins had fuel melting and central void formation but no internal fuel motion. The posttest condition of the fuel pins provided data for use in evaluating, improving, and validating computer models for predicting transient fuel pin behavior. 


\section{Summary of TREAT Test RFT-L2}

Performed 22 June 1983

Transient No. 2457

PRE-TEST SAMPLE CHARACTERIZATION
\begin{tabular}{|l|l|}
\hline \multicolumn{2}{|c|}{ FUEL } \\
\hline Form & $\mathrm{He}$-bonded $\mathrm{MOX}$ \\
\hline Composition & $\begin{array}{l}\text { Pin 1: } \mathrm{UO}_{2}-27 \% \mathrm{PuO}_{2} \\
\text { Pin 2: } \mathrm{UO}_{2}-22 \% \mathrm{PuO}_{2} \\
\text { Pin 3: } \mathrm{UO}_{2}-27 \% \mathrm{PuO}_{2} \\
\left.\text { (Natural } \mathrm{UO}_{2}, \mathrm{O} / \mathrm{M}=1.96\right)\end{array}$ \\
\hline Geometry & Solid pellet \\
\hline Fissile height & $91.4 \mathrm{~cm}$ \\
\hline $\begin{array}{l}\text { Smear } \\
\text { density }\end{array}$ & $85 \%$ of theoretical \\
\hline
\end{tabular}

\begin{tabular}{|l|l|}
\hline \multicolumn{2}{|c|}{ CLADDING } \\
\hline Material & SS $316(20 \% \mathrm{CW})$ \\
\hline OD & $5.84 \mathrm{~mm}$ \\
\hline Thickness & $0.38 \mathrm{~mm}$ \\
\hline
\end{tabular}

\begin{tabular}{|c|c|}
\hline \multicolumn{2}{|c|}{ PRE-IRRADIATION CHARACTERISTICS } \\
\hline BU (peak axial) & $\begin{array}{l}\text { Pin } 1: 1.5 \mathrm{MWd} / \mathrm{kg} \\
\text { Pin 2: } 2.3 \mathrm{MWd} / \mathrm{kg} \\
\text { Pin 3: } 1.5 \mathrm{MWd} / \mathrm{kg}\end{array}$ \\
\hline Peak linear power & $\begin{array}{l}\text { Pin 1: 27 kW/m } \\
\text { Pin 2: } \sim 41 \mathrm{~kW} / \mathrm{m} \\
\text { Pin } 3: \sim 26 \mathrm{~kW} / \mathrm{m}\end{array}$ \\
\hline $\begin{array}{l}\text { Peak internal clad } \\
\text { temperature }\end{array}$ & $\begin{array}{l}\text { Pin } 1: 522^{\circ} \mathrm{C} \\
\text { Pin } 2: 603^{\circ} \mathrm{C} \\
\text { Pin } 3: 516^{\circ} \mathrm{C}\end{array}$ \\
\hline $\begin{array}{l}\text { Fluence } \\
\text { (spectrum) }\end{array}$ & $\begin{array}{l}\text { Fluence not available; fast } \\
\text { spectrum }\end{array}$ \\
\hline
\end{tabular}

\begin{tabular}{|l|l|}
\hline \multicolumn{2}{|c|}{ TEST CONDITIONS, MEASUREMENTS, \& ANALYSES } \\
\hline $\begin{array}{l}\text { Coolant } \\
\text { environment }\end{array}$ & Flowing sodium \\
\hline $\begin{array}{l}\text { Transient } \\
\text { conditions }\end{array}$ & $\begin{array}{l}\text { Short ( 7 s) preheat at pin powers between 1X } \\
\text { and 2x nominal, then 5 } \text { d/s overpower transient } \\
\text { reaching pin powers of 3.7 times nominal (Pin 1), } \\
\text { 2.0 times nominal (Pin 2), and 3.4 times nominal } \\
\text { (Pin 3) }\end{array}$ \\
\hline $\begin{array}{l}\text { Fuel sample } \\
\text { configuration } \\
\text { in the test }\end{array}$ & Three pins, each in a separate flowtube \\
\hline $\begin{array}{l}\text { Test vehicle \& } \\
\text { experiment } \\
\text { system }\end{array}$ & $\begin{array}{l}\text { Mark-IIIA sodium loop, with axial flux-shaping } \\
\text { collars }\end{array}$ \\
\hline $\begin{array}{l}\text { Measurements } \\
\text { /diagnostics } \\
\text { during test }\end{array}$ & Thermocouples, flowmeters \\
\hline $\begin{array}{l}\text { Posttest } \\
\text { coolant flow } \\
\text { condition }\end{array}$ & Essentially the same as pretest. \\
\hline $\begin{array}{l}\text { Posttest } \\
\text { condition of } \\
\text { test sample(s) }\end{array}$ & All pins remained intact. \\
\hline $\begin{array}{l}\text { Posttest } \\
\text { measurements } \\
\text { \& information }\end{array}$ & $\begin{array}{l}\text { Macro-examination, neutron radiography, } \\
\text { gamma scanning, fission gas sampling, } \\
\text { ceramographic examination }\end{array}$ \\
\hline $\begin{array}{l}\text { Posttest } \\
\text { analyses }\end{array}$ & $\begin{array}{l}\text { Analyses using TEMECH, SAS4A, FPIN2, and } \\
\text { LIFE codes }\end{array}$ \\
\hline
\end{tabular}

\section{OTHER FUEL SAMPLE INFORMATION}

FFTF driver fuel pins; $145 \mathrm{~mm}$ long Inconel reflector above upper insulator pellets

\section{KEY SUMMARY INFORMATION ABOUT THE TEST}

Test RFT-L3 was conducted to investigate the effects of a $50 \phi / s$ unprotected overpower transient on very low burnup FFTF driver fuel pins that had been irradiated at different power levels. The test conditions were selected to cause fuel melting and cladding strain but not cladding failure. Peak power reached in the fuel was 3.4-3.7 times nominal in Pins 1 and 3 (the two lower-power-irradiated pins) and 2.0 times nominal in Pin 2 (the higher-power-irradiated pin). The maximum measured strains were $0.56 \%$ in Pin 1, none in Pin 2, and $0.14 \%$ in Pin 3 . All pins had fuel melting and central void formation. The maximum melt radius was $\sim 0.75 \mathrm{r} / \mathrm{R}$ in Pins 1 and 3 and $\sim 0.60 \mathrm{r} / \mathrm{R}$ in Pin 2, all peaking at a height $\mathrm{x} / \mathrm{L} \sim 0.6$. The posttest condition of the fuel pins provided data for use in evaluating, improving, and validating computer models for predicting transient fuel pin 


\title{
Summary of TREAT Test RFT-L3
}

\author{
Performed 30 January 1984
}

Transient No. 2531

\begin{tabular}{l}
\hline PRE-TEST SAMPLE CHARACTERIZATION \\
\begin{tabular}{|l|l|}
\hline \multicolumn{2}{|c|}{ FUEL } \\
\hline Form & $\mathrm{He}$-bonded $\mathrm{MOX}$ \\
\hline Composition & $\begin{array}{l}\mathrm{UO}_{2}-23 \% \mathrm{PuO}_{2} \\
\left.\text { (Natural } \mathrm{UO}_{2}, \mathrm{O} / \mathrm{M}=1.96\right)\end{array}$ \\
\hline Geometry & Solid pellet \\
\hline Fissile height & $91.4 \mathrm{~cm}$ \\
\hline $\begin{array}{l}\text { Smear } \\
\text { density }\end{array}$ & $85 \%$ of theoretical \\
\hline
\end{tabular}
\end{tabular}

\begin{tabular}{|l|l|}
\hline \multicolumn{2}{|c|}{ CLADDING } \\
\hline Material & SS $316(20 \% \mathrm{CW})$ \\
\hline OD & $5.82 \mathrm{~mm}$ \\
\hline Thickness & $0.38 \mathrm{~mm}$ \\
\hline
\end{tabular}

\begin{tabular}{|l|l|}
\hline \multicolumn{2}{|c|}{ TEST CONDITIONS, MEASUREMENTS, \& ANALYSES } \\
\hline $\begin{array}{l}\text { Coolant } \\
\text { environment }\end{array}$ & Flowing sodium \\
\hline $\begin{array}{l}\text { Transient } \\
\text { conditions }\end{array}$ & $\begin{array}{l}\text { 7-s preheat at steady power, then } 5 \text { c/s } \\
\text { overpower ramp for } ~ 13 \text { s reaching peak pin } \\
\text { then scram }\end{array}$ \\
\hline $\begin{array}{l}\text { Fuel sample } \\
\text { configuration } \\
\text { in the test }\end{array}$ & Three pins, each in a separate flowtube \\
\hline $\begin{array}{l}\text { Test vehicle \& } \\
\text { experiment } \\
\text { system }\end{array}$ & $\begin{array}{l}\text { Mark-IIIA sodium loop, with axial flux-shaping } \\
\text { collars }\end{array}$ \\
\hline $\begin{array}{l}\text { Measurements } \\
\text { /diagnostics } \\
\text { during test }\end{array}$ & Thermocouples, flowmeters \\
\hline $\begin{array}{l}\text { Posttest } \\
\text { coolant flow } \\
\text { condition }\end{array}$ & Essentially the same as pretest. \\
\hline $\begin{array}{l}\text { Posttest } \\
\text { condition of } \\
\text { test sample(s) }\end{array}$ & No cladding failures. \\
\hline $\begin{array}{l}\text { Posttest } \\
\text { measurements } \\
\text { \& information }\end{array}$ & $\begin{array}{l}\text { Neutron radiography, gamma scanning, } \\
\text { profilometry, destruction examination }\end{array}$ \\
\hline $\begin{array}{l}\text { Posttest } \\
\text { analyses }\end{array}$ & Analyses with SAS4A and TEMECH \\
\hline
\end{tabular}

\begin{tabular}{|l|l|}
\hline \multicolumn{2}{|l|}{ PRE-IRRADIATION CHARACTERISTICS } \\
\hline BU (peak axial) & $\begin{array}{l}\text { Pin 1: } 2.6 \% \mathrm{HM} \\
\text { Pin 2: } 5.2 \% \mathrm{HM} \\
\text { Pin 3: } 5.2 \% \mathrm{HM}\end{array}$ \\
\hline Peak linear power & $\begin{array}{l}\text { Pin 1: } \sim 35 \mathrm{~kW} / \mathrm{m} \\
\text { Pin 2: } \sim 36 \mathrm{~kW} / \mathrm{m} \\
\text { Pin 3: } \sim 38 \mathrm{~kW} / \mathrm{m}\end{array}$ \\
\hline $\begin{array}{l}\text { Peak internal clad } \\
\text { temperature }\end{array}$ & $\begin{array}{l}\text { Pin } 1: 463^{\circ} \mathrm{C} \\
\text { Pin 2: } 485^{\circ} \mathrm{C} \\
\text { Pin 3: } 481^{\circ} \mathrm{C}\end{array}$ \\
\hline Fluence & Pin 1: $0.418 \times 10^{23} \mathrm{n} / \mathrm{cm}^{2}$ \\
(spectrum) & Pin 2: $0.869 \times 10^{23} \mathrm{n} / \mathrm{cm}^{2}$ \\
& Pin 3: $0.870 \times 10^{23} \mathrm{n} / \mathrm{cm}^{2}$ \\
& Fast spectrum \\
\hline
\end{tabular}

\section{OTHER FUEL SAMPLE INFORMATION}

FFTF driver fuel pins; $145 \mathrm{~mm}$ long Inconel reflector above upper insulator pellets

\begin{tabular}{l}
\hline KEY SUMMARY INFORMATION ABOUT THE TEST \\
\hline Test RFT-L3 was conducted to investigate the effects of a $5 \phi / s$ unprotected overpower transient on low-to moderate burnup \\
irradiated FFTF driver fuel pins. The test conditions were selected to cause fuel melting and cladding strain but no cladding \\
failure. The fuel reached about 1.8 times nominal power, resulting in fuel melting and central void formation. Measurable \\
cladding strain occurred only in the lower-burnup pin. The posttest condition of the fuel pins provided data for use in \\
evaluating, improving, and validating computer models for predicting transient fuel pin behavior.
\end{tabular}




\title{
Summary of TREAT Test RFT-L4
}

\author{
Performed 9 February 1984
}

Transient No. 2535

PRE-TEST SAMPLE CHARACTERIZATION
\begin{tabular}{|l|l|}
\hline \multicolumn{2}{|c|}{ FUEL } \\
\hline Form & $\mathrm{He}$-bonded $\mathrm{MOX}$ \\
\hline Composition & $\mathrm{UO}_{2}-22 \% \mathrm{PuO}_{2}$ \\
\hline Geometry & Solid pellet \\
\hline Fissile height & $91.4 \mathrm{~cm}$ \\
\hline $\begin{array}{l}\text { Smear } \\
\text { density }\end{array}$ & $85 \%$ of theoretical \\
\hline
\end{tabular}

\begin{tabular}{|l|l|}
\hline \multicolumn{2}{|c|}{ TEST CONDITIONS, MEASUREMENTS, \& ANALYSES } \\
\hline $\begin{array}{l}\text { Coolant } \\
\text { environment }\end{array}$ & Flowing sodium \\
\hline $\begin{array}{l}\text { Transient } \\
\text { conditions }\end{array}$ & $\begin{array}{l}\sim 6.2-s \text { preheat at steady power, then } 1 \text { \$/s } \\
\text { overpower ramp for } ~ 125 \text { s reaching peak pin } \\
\text { powers of } 265-298 \mathrm{~kW} / \mathrm{m} \text { ( } 6.8 \text { times nominal), } \\
\text { then scram }\end{array}$ \\
\hline $\begin{array}{l}\text { Fuel sample } \\
\text { configuration } \\
\text { in the test }\end{array}$ & Three pins in separate flowtubes \\
\hline $\begin{array}{l}\text { Test vehicle \& } \\
\text { experiment } \\
\text { system }\end{array}$ & $\begin{array}{l}\text { Mark-IIIA sodium loop, with axial flux-shaping } \\
\text { collars }\end{array}$ \\
\hline $\begin{array}{l}\text { Measurements } \\
\text { /diagnostics } \\
\text { during test }\end{array}$ & Thermocouples, flowmeters \\
\hline $\begin{array}{l}\text { Posttest } \\
\text { coolant flow } \\
\text { condition }\end{array}$ & Essentially the same as pretest. \\
\hline $\begin{array}{l}\text { Posttest } \\
\text { condition of } \\
\text { test sample(s) }\end{array}$ & No cladding failures. \\
\hline $\begin{array}{l}\text { Posttest } \\
\text { measurements } \\
\text { \& information }\end{array}$ & $\begin{array}{l}\text { Gamma scanning, neutron radiography, } \\
\text { profilometry, destructive examinations }\end{array}$ \\
\hline $\begin{array}{l}\text { Posttest } \\
\text { analyses }\end{array}$ & Analysis with SAS4A and TEMECH codes. \\
\hline
\end{tabular}

\section{OTHER FUEL SAMPLE INFORMATION}

FFTF driver fuel pins; $145 \mathrm{~mm}$ long Inconel reflector above upper insulator pellets

\begin{tabular}{|l|l|}
\hline \multicolumn{2}{|l|}{ PRE-IRRADIATION CHARACTERISTICS } \\
\hline BU (peak axial) & $\begin{array}{l}\text { Pin 1: } 2.6 \% \mathrm{HM} \\
\text { Pin 2: } 5.4 \% \mathrm{HM} \\
\\
\text { Pin 3: } 5.3 \% \mathrm{HM}\end{array}$ \\
\hline Peak linear power & $\begin{array}{l}\text { Pin } 1: 35 \mathrm{~kW} / \mathrm{m} \\
\text { Pin 2: } 36 \mathrm{~kW} / \mathrm{m} \\
\text { Pin 3: } 38 \mathrm{~kW} / \mathrm{m}\end{array}$ \\
\hline Peak internal clad & Pin 1:517 $\mathrm{C}$ \\
temperature & Pin 2: $520^{\circ} \mathrm{C}$ \\
& Pin 3: $530^{\circ} \mathrm{C}$ \\
\hline Fluence & Pin $1: 0.423 \times 10^{23} \mathrm{n} / \mathrm{cm}^{2}$ \\
(spectrum) & Pin 2: $0.877 \times 10^{23} \mathrm{n} / \mathrm{cm}^{2}$ \\
& Pin 3: $0.872 \times 10^{23} \mathrm{n} / \mathrm{cm}^{2}$ \\
& Fast spectrum \\
\hline
\end{tabular}

\section{KEY SUMMARY INFORMATION ABOUT THE TEST}

Test RFT-L4 was conducted to investigate the effects of a $1 \$ / s$ unprotected overpower transient on low to intermediate burnup FFTF driver fuel pins. The test conditions were selected to cause fuel melting and cladding strain but no cladding failure. The fuel pins reached about 6.8 times nominal power and experienced fuel melting, central void formation, and (in the lower burnup pin only) measurable cladding strain. The posttest condition of the fuel pins provided data for use in evaluating, improving, and validating computer models for predicting transient fuel pin behavior. 


\section{Appendix K: One-page Summaries of STEP-series Tests}

One-page summaries of each of the STEP-series tests are included here. These summaries can also be found in TREXR [6]. 


\section{Summary of TREAT Test STEP1 \\ Performed 19 June 1984 \\ Transient No. 2568}

\section{PRE-TEST SAMPLE CHARACTERIZATION}

\begin{tabular}{|l|l|}
\hline \multicolumn{2}{|c|}{ FUEL } \\
\hline Form & He-bonded cm uranium dioxide \\
\hline Composition & $\begin{array}{l}\mathrm{UO}_{2} \\
8.26 \mathrm{wt} \%{ }^{235} \mathrm{U} \text { in U (as fabricated) }\end{array}$ \\
\hline Geometry & Solid pellet stack inside cladding \\
\hline Fissile height & $100 \mathrm{~cm}$ \\
\hline $\begin{array}{l}\text { Smear } \\
\text { density }\end{array}$ & $9.77 \mathrm{~g} / \mathrm{cm}^{3}$ \\
\hline
\end{tabular}

\begin{tabular}{|l|l|}
\hline \multicolumn{2}{|c|}{ CLADDING } \\
\hline Material & Zircaloy-4 \\
\hline OD & $0.950 \mathrm{~cm}$ \\
\hline Thickness & 0.063 \\
\hline
\end{tabular}

\begin{tabular}{|l|l|}
\hline PRE-IRRADIATION CHARACTERISTICS \\
\hline Burnup & $\begin{array}{l}33.1-36.4 \mathrm{GWd} / \mathrm{T} \text { (axial } \\
\text { average) }\end{array}$ \\
\hline Peak linear power & $21.9-26.7 \mathrm{~kW} / \mathrm{m}$ \\
\hline $\begin{array}{l}\text { Peak internal clad } \\
\text { temperature }\end{array}$ & (not available) \\
\hline $\begin{array}{l}\text { Fluence } \\
\text { (spectrum) }\end{array}$ & $\begin{array}{l}\text { Fluence not available; thermal } \\
\text { spectrum }\end{array}$ \\
\hline
\end{tabular}

\section{OTHER FUEL SAMPLE INFORMATION}

BR-3 elements from Belgonucleaire

Fill gas: He at $20 \mathrm{~atm}$

\begin{tabular}{|l|l|}
\hline \multicolumn{2}{|c|}{ TEST CONDITIONS, MEASUREMENTS, \& ANALYSES } \\
\hline $\begin{array}{l}\text { Coolant } \\
\text { environment }\end{array}$ & Fowing steam \\
\hline $\begin{array}{l}\text { Transient } \\
\text { conditions }\end{array}$ & $\begin{array}{l}20.6 \text { min. at low pressure with an initial heatup } \\
\text { rate of } 1.8 \mathrm{~K} / \mathrm{s}\end{array}$ \\
\hline $\begin{array}{l}\text { Fuel sample } \\
\text { configuration } \\
\text { in the test }\end{array}$ & 4-pin bundle \\
\hline $\begin{array}{l}\text { Test vehicle \& } \\
\text { experiment } \\
\text { system }\end{array}$ & $\begin{array}{l}\text { Single-pass steam system, fission-product } \\
\text { sampling system, fission gas and hydrogen } \\
\text { collection systems }\end{array}$ \\
\hline $\begin{array}{l}\text { Measurements } \\
\text { /diagnostics } \\
\text { during test }\end{array}$ & $\begin{array}{l}\text { Pressures, temperature profiles, steam flow rate, } \\
\text { hydrogen concentration, fuel motion } \\
\text { (hodoscope) }\end{array}$ \\
\hline $\begin{array}{l}\text { Posttest } \\
\text { coolant flow } \\
\text { condition }\end{array}$ & $\begin{array}{l}\text { Fission product build-up caused partial, } \\
\text { temporary blockage }\end{array}$ \\
\hline $\begin{array}{l}\text { Posttest } \\
\text { condition of } \\
\text { test sample(s) }\end{array}$ & $\begin{array}{l}\text { Severe fuel damage over } 90 \% \text { of the fuel column } \\
\text { (in central \& upper part) }\end{array}$ \\
\hline $\begin{array}{l}\text { Posttest } \\
\text { measurements } \\
\text { \& info }\end{array}$ & $\begin{array}{l}\text { SEM examination, electron microprobe, neutron } \\
\text { radiography, gamma ray scanning, X-ray } \\
\text { diffraction, mass spectrometry, chemical } \\
\text { composition analysis, particle size classification }\end{array}$ \\
\hline $\begin{array}{l}\text { Posttest } \\
\text { analyses }\end{array}$ & $\begin{array}{l}\text { SOAP computer code validation, fission product } \\
\text { analysis, thermodynamic analysis, thermal- } \\
\text { hydraulics, hydrogen generation and release }\end{array}$ \\
\hline
\end{tabular}

\section{KEY SUMMARY INFORMATION ABOUT THE TEST}

STEP-1 investigated the release of fission products and the interaction between steam and fission products during the simulation of a PWR large-break loss-of-coolant accident with failure of emergency-core cooling injection. Calculations indicate a peak fuel temperature of $2900 \mathrm{~K}$. Hydrogen generation corresponded to approximately $90 \%$ cladding oxidation. Damage was severe throughout the central and top portions of the fuel column. An early, large release of volatile fission products was observed at relatively low temperatures, believed to have been associated with the cladding ballooning that occurred. Fuel motion was overall upward, with some fuel movement toward the midplane during the time of vigorous oxidation. Partial blockage of released material occurred, which was spontaneously relieved. Fission product deposits collected during the transient were composed mainly of cesium, tin, molybdenum, rubidium, and possible silicon. It was found that the chemistry of fission-product deposits was complex and that fuel and cladding particles were released by mechanical processes. 


\section{Summary of TREAT Test STEP2 \\ Performed 20 November 1984}

Transient No. 2586

\section{PRE-TEST SAMPLE CHARACTERIZATION}

\begin{tabular}{|l|l|}
\hline \multicolumn{2}{|c|}{ FUEL } \\
\hline Form & He-bonded uranium dioxide \\
\hline Composition & $\begin{array}{l}\mathrm{UO}_{2} \\
8.26 \mathrm{wt} \%{ }^{235} \mathrm{U} \text { in U (as fabricated) }\end{array}$ \\
\hline Geometry & Solid pellet stack inside cladding \\
\hline Fissile height & $100 \mathrm{~cm}$ \\
\hline $\begin{array}{l}\text { Smear } \\
\text { density }\end{array}$ & $9.77 \mathrm{~g} / \mathrm{cm}^{3}$ \\
\hline
\end{tabular}

\begin{tabular}{|l|l|}
\hline \multicolumn{2}{|c|}{ CLADDING } \\
\hline Material & Zircaloy-4 \\
\hline OD & $0.950 \mathrm{~cm}$ \\
\hline Thickness & $0.063 \mathrm{~cm}$ \\
\hline
\end{tabular}

\begin{tabular}{|l|l|}
\hline \multicolumn{2}{|l|}{ PRE-IRRADIATION CHARACTERISTICS } \\
\hline Burnup & $30.6 \mathrm{GWdT}$ (axial average) \\
\hline Peak linear power & $30.1-30.2 \mathrm{~kW} / \mathrm{m}$ \\
\hline $\begin{array}{l}\text { Peak internal clad } \\
\text { temperature }\end{array}$ & (not available) \\
\hline $\begin{array}{l}\text { Fluence } \\
\text { (spectrum) }\end{array}$ & $\begin{array}{l}\text { Fluence not available; thermal } \\
\text { spectrum }\end{array}$ \\
\hline
\end{tabular}

\section{OTHER FUEL SAMPLE INFORMATION}

BR-3 elements from Belgonucleaire

Fill gas: He at $20 \mathrm{~atm}$

\begin{tabular}{|c|c|}
\hline \multicolumn{2}{|c|}{ TEST CONDITIONS, MEASUREMENTS, \& ANALYSES } \\
\hline $\begin{array}{l}\text { Coolant } \\
\text { environment }\end{array}$ & Flowing steam \\
\hline $\begin{array}{l}\text { Transient } \\
\text { conditions }\end{array}$ & $\begin{array}{l}20.5 \text { minutes at low pressure with a fuel heatup } \\
\text { rate of } 2.1 \mathrm{~K} / \mathrm{s} \text { (slowest achievable in test but } \\
\text { intended to simulate an accident having an initial } \\
\text { heatup rate of } 0.3 \mathrm{~K} / \mathrm{s} \text { ) }\end{array}$ \\
\hline $\begin{array}{l}\text { Fuel sample } \\
\text { configuration } \\
\text { in the test }\end{array}$ & 4-pin bunde \\
\hline $\begin{array}{l}\text { Test vehicle \& } \\
\text { experiment } \\
\text { system }\end{array}$ & $\begin{array}{l}\text { Single-pass steam system; fission-product } \\
\text { sampling system, fission gas and hydrogen } \\
\text { collection systems }\end{array}$ \\
\hline $\begin{array}{l}\text { Measurements } \\
\text { /diagnostics } \\
\text { during test }\end{array}$ & $\begin{array}{l}\text { Pressures, temperature profiles, steam flow rate, } \\
\text { hydrogen concentration, fuel motion } \\
\text { (hodoscope) }\end{array}$ \\
\hline $\begin{array}{l}\text { Posttest } \\
\text { coolant flow } \\
\text { condition }\end{array}$ & Fission product build-up caused partial blockage \\
\hline $\begin{array}{l}\text { Posttest } \\
\text { condition of } \\
\text { test sample(s) }\end{array}$ & $\begin{array}{l}\text { Extensive fuel damage in the upper three-fourths } \\
\text { of the fuel column }\end{array}$ \\
\hline $\begin{array}{l}\text { Posttest } \\
\text { measurements } \\
\text { \& info }\end{array}$ & $\begin{array}{l}\text { SEM examination, electron microprobe, neutron } \\
\text { radiography, gamma ray scanning, X-ray } \\
\text { diffraction, mass spectrometry, chemical } \\
\text { composition analysis, and particle size } \\
\text { classification }\end{array}$ \\
\hline $\begin{array}{l}\text { Posttest } \\
\text { analyses }\end{array}$ & $\begin{array}{l}\text { SOAP computer code validation, fission product } \\
\text { analysis, thermodynamic analysis, thermal- } \\
\text { hydraulics, hydrogen generation and release }\end{array}$ \\
\hline
\end{tabular}

\section{KEY SUMMARY INFORMATION ABOUT THE TEST}

STEP-2 investigated the release of fission products and the interaction between steam and fission products during the simulation of a BWR accident caused by failure of the high-pressure emergency core cooling and long-term decay heat removal systems, with automatic depressurization and temporary low pressure coolant injection assumed to occur. Calculations indicate a peak fuel temperature of $2700 \mathrm{~K}$. Hydrogen generation corresponded to approximately $70 \%$ cladding oxidation. Damage was severe throughout the central and top portions of the fuel column. An early and partial blockage of released material occurred which (unlike in STEP-1) was not spontaneously relieved, so the test was terminated before the pressure increase could have caused a facilities problem. A large release of fission products was observed at relatively low temperatures, believed to have been caused by cladding ballooning. Fission product deposits during the transient were composed mainly of cesium, tin, molybdenum, rubidium, and silicon, accompanied by tellurium. lodine was co-located with high levels of nickel and no cesium. It was found that the chemistry of fission-product deposits was complex and that fuel and cladding particles were released by mechanical processes. 


\section{Summary of TREAT Test STEP3 \\ Performed 30 January 1985}

Transient No. 2588

\section{PRE-TEST SAMPLE CHARACTERIZATION}

\begin{tabular}{|l|l|}
\hline \multicolumn{2}{|c|}{ FUEL } \\
\hline Form & He-bonded uranium dioxide \\
\hline Composition & $\begin{array}{l}\mathrm{UO}_{2} \\
8.26 \mathrm{wt} \%{ }^{235} \mathrm{U} \text { in U (as fabricated) }\end{array}$ \\
\hline Geometry & Solid pellet stack inside cladding \\
\hline Fissile height & $100 \mathrm{~cm}$ \\
\hline $\begin{array}{l}\text { Smear } \\
\text { density }\end{array}$ & $9.77 \mathrm{~g} / \mathrm{cm}^{3}$ \\
\hline
\end{tabular}

\begin{tabular}{|l|l|}
\hline \multicolumn{2}{|c|}{ CLADDING } \\
\hline Material & Zircal oy-4 \\
\hline OD & $0.950 \mathrm{~cm}$ \\
\hline Thickness & $0.063 \mathrm{~cm}$ \\
\hline
\end{tabular}

\begin{tabular}{|l|l|}
\hline PRE-IRRADIATION CHARACTERISTICS \\
\hline Burnup & $\begin{array}{l}35.2-36.0 \mathrm{GWd} / \mathrm{T} \text { (axial } \\
\text { average) }\end{array}$ \\
\hline Peak linear power & $33.6-34.2 \mathrm{~kW} / \mathrm{m}$ \\
\hline $\begin{array}{l}\text { Peak internal clad } \\
\text { temperature }\end{array}$ & (not available) \\
\hline $\begin{array}{l}\text { Fluence } \\
\text { (spectrum) }\end{array}$ & $\begin{array}{l}\text { Fluence not available; thermal } \\
\text { spectrum }\end{array}$ \\
\hline
\end{tabular}

\section{OTHER FUEL SAMPLE INFORMATION}

BR-3 elements from Belgonucleaire

Fill gas: He at $20 \mathrm{~atm}$

\begin{tabular}{|c|c|}
\hline \multicolumn{2}{|c|}{ TEST CONDITIONS, MEASUREMENTS, \& ANALYSES } \\
\hline $\begin{array}{l}\text { Coolant } \\
\text { environment }\end{array}$ & Flowing steam \\
\hline $\begin{array}{l}\text { Transient } \\
\text { conditions }\end{array}$ & $\begin{array}{l}21.4 \mathrm{~min} \text {. at high pressure with heatup rate of } \\
1.1 \mathrm{~K} / \mathrm{s}\end{array}$ \\
\hline $\begin{array}{l}\text { Fuel sample } \\
\text { configuration } \\
\text { in the test }\end{array}$ & 4-pin bundle \\
\hline $\begin{array}{l}\text { Test vehicle \& } \\
\text { experiment } \\
\text { system }\end{array}$ & $\begin{array}{l}\text { Single-pass steam system, fission-product } \\
\text { sampling system, fission gas and hydrogen } \\
\text { collection systems }\end{array}$ \\
\hline $\begin{array}{l}\text { Measurements } \\
\text { /diagnostics } \\
\text { during test }\end{array}$ & $\begin{array}{l}\text { Pressures, temperature profiles, steam flow rate, } \\
\text { hydrogen concentration, fuel motion } \\
\text { (hodoscope) }\end{array}$ \\
\hline $\begin{array}{l}\text { Posttest } \\
\text { coolant flow } \\
\text { condition }\end{array}$ & No blockage \\
\hline $\begin{array}{l}\text { Posttest } \\
\text { condition of } \\
\text { test sample(s) }\end{array}$ & $\begin{array}{l}\text { Major fission product release; severe fuel } \\
\text { damage limited to upper third of fuel column }\end{array}$ \\
\hline $\begin{array}{l}\text { Posttest } \\
\text { measurements } \\
\text { \& info }\end{array}$ & $\begin{array}{l}\text { SEM examination, electron microprobe, neutron } \\
\text { radiography, gamma ray scanning, X-ray } \\
\text { diffraction, mass spectrometry, chemical } \\
\text { composition analysis, and particle size } \\
\text { classification }\end{array}$ \\
\hline $\begin{array}{l}\text { Posttest } \\
\text { analyses }\end{array}$ & $\begin{array}{l}\text { SOAP computer code validation, fission product } \\
\text { analysis, thermodynamic analysis, thermal- } \\
\text { hydraulics, hydrogen generation and release }\end{array}$ \\
\hline
\end{tabular}

\footnotetext{
KEY SUMMARY INFORMATION ABOUT THE TEST

STEP-3 investigated the release of fission products and the interaction between steam and fission products during the simulation of a PWR station blackout by creating transient conditions typical of the failure of the feedwater system combined with failure to recover station electrical power. Calculations indicate a peak fuel temperature of $2200 \mathrm{~K}$, which was lower than expected. Hydrogen generation corresponded to approximately $40 \%$ cladding oxidation. Damage was severe over the upper three-fourths of the fuel column. Fuel movement was overall upward. A smaller release of fission products occurred in this high pressure test than in the low-pressure tests STEP-1 and -2 because convective flow was induced due to the high pressure conditions, resulting in lower fuel temperatures. Cesium, rubidium, and a trace of iodine were the only fission products found in the deposits. However, the test also demonstrated the tendency for component elements of ceramic and stainless steel structures to be volatilized and transported in high pressure steam. Aerosol deposits were found to contain mainly silicon and iron.
} 


\section{Summary of TREAT Test STEP4 \\ Conducted 2 March 1985 \\ Transient No. 2590}

\section{PRE-TEST SAMPLE CHARACTERIZATION}

\begin{tabular}{|l|l|}
\hline \multicolumn{2}{|c|}{ FUEL } \\
\hline Form & He-bonded uranium dioxide \\
\hline Composition & $\begin{array}{l}\mathrm{UO}_{2} \\
8.26 \mathrm{wt} \%{ }^{235} \mathrm{U} \text { in U (as fabricated) }\end{array}$ \\
\hline Geometry & Solid pellet stack inside cladding \\
\hline Fissile height & $100 \mathrm{~cm}$ \\
\hline $\begin{array}{l}\text { Smear } \\
\text { density }\end{array}$ & $9.77 \mathrm{~g} / \mathrm{cm}^{3}$ \\
\hline
\end{tabular}

\begin{tabular}{|l|l|}
\hline \multicolumn{2}{|c|}{ CLADDING } \\
\hline Material & Zircal oy-4 \\
\hline OD & $0.950 \mathrm{~cm}$ \\
\hline Thickness & $0.063 \mathrm{~cm}$ \\
\hline
\end{tabular}

\begin{tabular}{|l|l|}
\hline PRE-IRRADIATION CHARACTERISTICS \\
\hline Burnup & $\begin{array}{l}34.9-36.0 \mathrm{GWd} / \mathrm{T} \text { (axial } \\
\text { average) }\end{array}$ \\
\hline Peak linear power & $33.6-34.5 \mathrm{~kW} / \mathrm{m}$ \\
\hline $\begin{array}{l}\text { Peak internal clad } \\
\text { temp. }\end{array}$ & (not available) \\
\hline $\begin{array}{l}\text { Fluence } \\
\text { (spectrum) }\end{array}$ & $\begin{array}{l}\text { Fluence not available; thermal } \\
\text { spectrum }\end{array}$ \\
\hline
\end{tabular}

\section{OTHER FUEL SAMPLE INFORMATION}

BR-3 elements from Belgonucleaire

Fill gas: $\mathrm{He}$ at $20 \mathrm{~atm}$

\begin{tabular}{|c|c|}
\hline \multicolumn{2}{|c|}{ TEST CONDITIONS, MEASUREMENTS, \& ANALYSES } \\
\hline $\begin{array}{l}\text { Coolant } \\
\text { environment }\end{array}$ & Flowing steam \\
\hline $\begin{array}{l}\text { Transient } \\
\text { conditions }\end{array}$ & $\begin{array}{l}23.8 \mathrm{~min} \text {. at high pressure ( } 8 \mathrm{MPa} \text { ) with a fast } \\
\text { heatup rate (initially } 1.0 \mathrm{~K} / \mathrm{s} \text { ) }\end{array}$ \\
\hline $\begin{array}{l}\text { Fuel sample } \\
\text { configuration } \\
\text { in the test }\end{array}$ & $\begin{array}{l}\text { 4-pin bundle; control rod material present (to } \\
\text { simulate PWR control rod) }\end{array}$ \\
\hline $\begin{array}{l}\text { Test vehicle \& } \\
\text { experiment } \\
\text { system }\end{array}$ & $\begin{array}{l}\text { Single-pass steam system, fission-product } \\
\text { sampling system, fission gas and hydrogen } \\
\text { collection systems }\end{array}$ \\
\hline $\begin{array}{l}\text { Measurements } \\
\text { /diagnostics } \\
\text { during test }\end{array}$ & $\begin{array}{l}\text { Pressures, temperature profiles, steam flow rate, } \\
\text { hydrogen concentration, f fuel motion } \\
\text { (hodoscope) }\end{array}$ \\
\hline $\begin{array}{l}\text { Posttest } \\
\text { coolant flow } \\
\text { condition }\end{array}$ & No blockage \\
\hline $\begin{array}{l}\text { Posttest } \\
\text { condition of } \\
\text { test sample(s) }\end{array}$ & $\begin{array}{l}\text { Upper third of the control rod material melted } \\
\text { and flowed downward; severe fuel damage was } \\
\text { limited to the upper quarter of the fuel column. }\end{array}$ \\
\hline $\begin{array}{l}\text { Posttest } \\
\text { measurements } \\
\text { \& info }\end{array}$ & $\begin{array}{l}\text { SEM examination, electron microprobe, neutron } \\
\text { radiography, gamma ray scanning, } X \text {-ray } \\
\text { diffraction, mass spectrometry. }\end{array}$ \\
\hline $\begin{array}{l}\text { Posttest } \\
\text { analyses }\end{array}$ & $\begin{array}{l}\text { SOAP computer code validation, fission product } \\
\text { analysis, thermodynamic analysis, thermal- } \\
\text { hydraulics, hydrogen generation and release }\end{array}$ \\
\hline
\end{tabular}

\footnotetext{
KEY SUMMARY INFORMATION ABOUT THE TEST

STEP-4 investigated the release of fission products and the interaction between steam and fission products during the simulation of a PWR station blackout by creating transient conditions typical of the failure of the feedwater system and failure to recover electrical power. The test was planned to be a duplicate of STEP-3 except that it also contained a simulated silver-indium-cadmium PWR control rod. Calculations indicate a peak fuel temperature below $2200 \mathrm{~K}$. Hydrogen generation corresponded to approximately $30 \%$ cladding oxidation. Fuel movement was overall upward. No fission products were found in the deposits. However, the test demonstrated the tendency for component elements of ceramic and stainless steel structures to be volatilized and transported in high pressure steam. Aerosol deposits were found to contain mainly iron with silicon.
} 


\section{Appendix L: One-page Summaries of R-series Tests}

One-page summaries of each of the R-series tests are included here. These summaries can also be found in TREXR [6]. 


\section{Summary of TREAT Test R-3}

Performed 19 October 1973

Transient No. 1526

\section{PRE-TEST SAMPLE CHARACTERIZATION}

\begin{tabular}{|l|l|}
\hline \multicolumn{2}{|c|}{ FUEL } \\
\hline Form & He-bonded oxide \\
\hline Composition & $\mathrm{UO}_{2,1} 14 \%{ }^{235} \mathrm{U}$ in U \\
\hline Geometry & Solid pellets with dished ends \\
\hline Fissile height & $\begin{array}{l}91.44 \mathrm{~cm} \text { (prototypic of FTR power } \\
\text { fuel design) }\end{array}$ \\
\hline $\begin{array}{l}\text { Smear } \\
\text { density }\end{array}$ & $9.4 \mathrm{~g} / \mathrm{cm}^{3}$ \\
\hline
\end{tabular}

\begin{tabular}{|l|l|}
\hline \multicolumn{2}{|c|}{ CLADDING } \\
\hline Material & $316 \mathrm{SS} 20 \% \mathrm{CW}$ \\
\hline OD & $0.584 \mathrm{~cm}$ \\
\hline Thickness & $0.038 \mathrm{~cm}$ \\
\hline
\end{tabular}

\begin{tabular}{|l|l|}
\hline \multicolumn{2}{|l|}{ PRE-IRRADIATION CHARACTERISTICS } \\
\hline BU (peak axial) & (Not pre-irradiated) \\
\hline Peak linear power & Not applicable \\
\hline $\begin{array}{l}\text { Peak internal clad } \\
\text { temperature }\end{array}$ & Not applicable \\
\hline $\begin{array}{l}\text { Fluence } \\
\text { (spectrum) }\end{array}$ & Not applicable \\
\hline
\end{tabular}

\section{OTHER FUEL SAMPLE INFORMATION}

$173 \mathrm{~g}$ fuel per pin. $14.4 \mathrm{~cm}$ upper and lower Inconel reflectors. 0.8-mm wire wrap.

\begin{tabular}{|c|c|}
\hline \multicolumn{2}{|c|}{ TEST CONDITIONS, MEASUREMENTS, \& ANALYSES } \\
\hline $\begin{array}{l}\text { Coolant } \\
\text { environment }\end{array}$ & $\begin{array}{l}316^{\circ} \mathrm{C} \text { sodium; flow at } 7.3 \mathrm{~m} / \mathrm{s} \text { for } 6 \mathrm{~s} \text { after } \\
\text { reaching steady power, then dropping rapidy to } \\
\text { a low level, followed by a more-gradual decay } \\
\text { until boiling began. }\end{array}$ \\
\hline $\begin{array}{l}\text { Transient } \\
\text { conditions }\end{array}$ & $\begin{array}{l}\text { Steady reactor power initially generating } 31 \\
\mathrm{~kW} / \mathrm{m} \text { average in the test fuel }\end{array}$ \\
\hline $\begin{array}{l}\text { Fuel sample } \\
\text { configuration } \\
\text { in the test }\end{array}$ & Single fuel pin \\
\hline $\begin{array}{l}\text { Test vehicle \& } \\
\text { experiment } \\
\text { system }\end{array}$ & $\begin{array}{l}\text { Gas-driven, once-through loop. Circular } \\
\text { stainless steel flowtube ( } 7.61 \mathrm{~mm} \text { ID, } 0.5 \text {-mm } \\
\text { thick) surrounded by a molybdenum reflector } \\
\text { tube ( } 27.4 \mathrm{~mm} I D, 1.5 \mathrm{~mm} \text { thick), with a } 9.4-\mathrm{mm} \\
\text { wide gas-filled annul us in between }\end{array}$ \\
\hline $\begin{array}{l}\text { Measurements } \\
\text { /diagnostics } \\
\text { during test }\end{array}$ & $\begin{array}{l}\text { Flowmeters, pressure transducers, } \\
\text { thermocouples, fuel motion detector } \\
\text { (hodoscope) }\end{array}$ \\
\hline $\begin{array}{l}\text { Posttest } \\
\text { coolant flow } \\
\text { condition }\end{array}$ & $\begin{array}{l}\text { Complete inlet blockage due to accumulation of } \\
\text { steel. }\end{array}$ \\
\hline $\begin{array}{l}\text { Posttest } \\
\text { condition of } \\
\text { test sample(s) }\end{array}$ & $\begin{array}{l}\text { A spiral-distorted column of bare fuel pellets, } \\
\text { which subsequently collapsed. }\end{array}$ \\
\hline $\begin{array}{l}\text { Posttest } \\
\text { measurements } \\
\text { \& information }\end{array}$ & Neutron radiography, macro-examination \\
\hline $\begin{array}{l}\text { Posttest } \\
\text { analyses }\end{array}$ & $\begin{array}{l}\text { Thermal-hydraulics, cladding motion and fuel } \\
\text { motion (SAS4A) }\end{array}$ \\
\hline
\end{tabular}

\section{KEY SUMMARY INFORMATION ABOUT THE TEST}

Test R3 was the first of the R-series tests and served as a proof test for subsequent seven-pin tests. (R1 and R2 were calibration and checkout tests). When local boiling began, the flow velocity had decreased to $1.8 \mathrm{~m} / \mathrm{s}(22 \%$ of the initial flow rate) and was decreasing at $12 \% / \mathrm{s}$. Inlet flow reversal occurred 1.7 s later. Relative to the time of flow reversal (reference time $=0$ ), the following sequence of events occurred: initial cladding failure at $1.2 \mathrm{~s}$, flowtube failure at $3.6 \mathrm{~s}$, and reactor power termination at $10.5 \mathrm{~s}$. (In locations where the flowtube had disappeared, radiant heat loss from hot fuel pins to the much-coder Mo tube became large. Significant oscillations of the inlet flow occurred after boiling began, including before inlet flow reversal. No significant pressure events were recorded. The hodoscope indicated that the fuel column moved into a helical shape in two phases, the first one limited radially by the cladding, and the second one limited radially by the Mo reflector. The fuel column was still upright when the reactor power was brought down, but when neutron radiographs were taken later, the fuel column had collapsed. Posttest examination revealed that over the length of the fuel column, almost all of the cladding, wire wrap, and flowtube had melted. A large mass of solidified steel had formed around the bottom insulator pellets of the fuel pin (just below the active fuel column) completely blocking the flow channel. Below the insulator pellets, the flowtube and cladding were essentially undamaged. A small amount of once-melted steel was located around the insulator pellets above the active fuel. 


\section{Summary of TREAT Test R-4}

Performed 9 November 1973

Transient No. 1527

\section{PRE-TEST SAMPLE CHARACTERIZATION}

\begin{tabular}{|l|l|}
\hline \multicolumn{2}{|c|}{ FUEL } \\
\hline Form & He-bonded oxide \\
\hline Composition & $\begin{array}{l}\mathrm{UO}_{2} \\
\text { Outer pins: } 14 \%{ }^{235} \mathrm{U} \text { in U } \\
\text { Inner pin: } 20 \%{ }^{225} \mathrm{U} \text { in U }\end{array}$ \\
\hline Geometry & Solid pellets with dished ends \\
\hline Fissile height & $\begin{array}{l}91.44 \mathrm{~cm} \text { (prototypic of FTR power } \\
\text { fuel design) }\end{array}$ \\
\hline $\begin{array}{l}\text { Smear } \\
\text { density }\end{array}$ & $9.4 \mathrm{~g} / \mathrm{cm}^{3}$ \\
\hline
\end{tabular}

\begin{tabular}{|l|l|}
\hline \multicolumn{2}{|c|}{ CLADDING } \\
\hline Material & $316 \mathrm{SS} 20 \% \mathrm{CW}$ \\
\hline OD & $0.584 \mathrm{~cm}$ \\
\hline Thickness & $0.038 \mathrm{~cm}$ \\
\hline
\end{tabular}

\begin{tabular}{|l|l|}
\hline \multicolumn{2}{|l|}{ PRE-IRRADIATION CHARACTERISTICS } \\
\hline BU (peak axial) & (Not pre-irradiated) \\
\hline Peak linear power & Not applicable \\
\hline $\begin{array}{l}\text { Peak internal clad } \\
\text { temperature }\end{array}$ & Not applicable \\
\hline $\begin{array}{l}\text { Fluence } \\
\text { (spectrum) }\end{array}$ & Not applicable \\
\hline
\end{tabular}

\section{OTHER FUEL SAMPLE INFORMATION}

$173 \mathrm{~g}$ fuel per pin. $14.4 \mathrm{~cm}$ upper and lower Inconel reflectors.

\begin{tabular}{|l|l|}
\hline \multicolumn{2}{|c|}{ TEST CONDITIONS, MEASUREMENTS, \& ANALYSES } \\
\hline $\begin{array}{l}\text { Coolant } \\
\text { environment }\end{array}$ & $\begin{array}{l}320^{\circ} \mathrm{C} \text { sodium; at } 7.5 \mathrm{~m} / \mathrm{s} \text { for } 3 \mathrm{~s} \text { after reaching } \\
\text { steady power, then gradual decrease to } 2.1 \mathrm{~m} / \mathrm{s} \\
\text { during } 2.1 \mathrm{~s} \text {, then gradual decrease to } 1.5 \mathrm{~m} / \mathrm{s} \\
\text { during } 4 \mathrm{~s} \text {, then decrease to zero in } 0.5 \mathrm{~s} .\end{array}$ \\
\hline $\begin{array}{l}\text { Transient } \\
\text { conditions }\end{array}$ & $\begin{array}{l}\text { Steady reactor power initially generating 31 } \\
\text { kW/m average in test-fuel bundle }\end{array}$ \\
\hline $\begin{array}{l}\text { Fuel sample } \\
\text { configuration } \\
\text { in the test }\end{array}$ & 7-pin hexagonal bunde \\
\hline $\begin{array}{l}\text { Test vehicle \& } \\
\text { experiment } \\
\text { system }\end{array}$ & $\begin{array}{l}\text { Gas-driven, once-through loop. The hexagonal } \\
0.5 \text {-mm thick stainless steel flowtube was } \\
\text { surrounded by a molybdenum tube, with an } ~ 2.5 \\
\text { mm intermediate gas gap. }\end{array}$ \\
\hline $\begin{array}{l}\text { Measurements } \\
\text { diagnostics } \\
\text { during test }\end{array}$ & $\begin{array}{l}\text { Flowmeters, pressure transducers, } \\
\text { thermocouples, fuel motion detector } \\
\text { (hodoscope) }\end{array}$ \\
\hline $\begin{array}{l}\text { Posttest } \\
\text { coolant flow } \\
\text { condition }\end{array}$ & Complete flow blockages at the inlet and outlet. \\
\hline $\begin{array}{l}\text { Posttest } \\
\text { condition of } \\
\text { test sample(s) }\end{array}$ & Gross meltdown of cladding and flowtube. \\
\hline $\begin{array}{l}\text { Posttest } \\
\text { measurements } \\
\text { \& information }\end{array}$ & Neutron radiography, macro-examination \\
\hline $\begin{array}{l}\text { Posttest } \\
\text { analyses }\end{array}$ & $\begin{array}{l}\text { Thermal-hydraulics, cladding motion and fuel } \\
\text { motion (SAS4A) }\end{array}$ \\
\hline
\end{tabular}

\section{KEY SUMMARY INFORMATION ABOUT THE TEST}

Test R4 focused on codant and cladding behavior under conditions representative of the central region of a high power-to-flow subassembly in the FTR (FFTF) beginning of life core, providing data to validate the SAS code voiding model. The objective was to allow the test to proceed as far into the destructive stage of the sequence as the TREAT limitation on integrated power would permit, well beyond the start of molten-fuel motion.

Sustained channel voiding began when the codant velocity had fallen to $1.83 \mathrm{~m} / \mathrm{s}$ and the flow decay rate was $12 \% / \mathrm{s}$. Relative to the time of boiling-induced inlet flow reversal (reference time $=0$ ), the following sequence of events occurred: initial cladding failure at 1.13 $\mathrm{s}$, upper coolant-channel blockage complete at $2.5 \mathrm{~s}$, flowtube fail ure at $3.14 \mathrm{~s}$ causing a major flow perturbation due to nitrogen gas entry from outside the flowtube, lower coolant-channel blockage complete at $5.20 \mathrm{~s}$, much fuel moved downward during the next $5 \mathrm{~s}$, and reactor power termination at $10.15 \mathrm{~s}$. No significant pressure events were recorded.

Posttest examination revealed that the steel cladding and flowtube was completely gone from the upper two-thirds of the initial testfuel region. A complete, $0.3-\mathrm{cm}$-thick steel planar blockage had formed at the upper insulator pellets. At the bottom, a 15-cm-thick steel blockage had formed extending radially to the molybdenum liner and extending downward $5 \mathrm{~cm}$ into the region of the lower reflector rods. A large mass of once-molten fuel was located on top of and partially buried within this blockage. Near the fuel midplane, fuel melting was complete, and most of the fuel had drained downward, leaving a thick, irregular crust on the wall of the Mo liner. In the upper part of the original fuel region, fuel melting was not complete, with only center melting of pellets. Although the molten fuel from the pellet centers had drained out, the sintered pellets remained in their stacked configuration in that region. The hodoscope data could not be processed. 


\section{Summary of TREAT Test R-5}

Performed 6 December 1973

Transient No. 1528

\section{PRE-TEST SAMPLE CHARACTERIZATION}

\begin{tabular}{|l|l|}
\hline \multicolumn{2}{|c|}{ FUEL } \\
\hline Form & He-bonded oxide \\
\hline Composition & $\begin{array}{l}\mathrm{UO}_{2} \\
\text { Outer pins: } 14 \%{ }^{235} \mathrm{U} \text { in U } \\
\text { Inner pin: } 20 \%{ }^{225} \mathrm{U} \text { in U }\end{array}$ \\
\hline Geometry & Solid pellets with dished ends \\
\hline Fissile height & $\begin{array}{l}91.44 \mathrm{~cm} \text { (prototypic of FTR power } \\
\text { fuel design) }\end{array}$ \\
\hline $\begin{array}{l}\text { Smear } \\
\text { density }\end{array}$ & $9.4 \mathrm{~g} / \mathrm{cm}^{3}$ \\
\hline
\end{tabular}

\begin{tabular}{|l|l|}
\hline \multicolumn{2}{|c|}{ CLADDING } \\
\hline Material & $316 \mathrm{SS} 20 \% \mathrm{CW}$ \\
\hline OD & $0.584 \mathrm{~cm}$ \\
\hline Thickness & $0.038 \mathrm{~cm}$ \\
\hline
\end{tabular}

\begin{tabular}{|l|l|}
\hline \multicolumn{2}{|l|}{ PRE-IRRADIATION CHARACTERISTICS } \\
\hline BU (peak axial) & (Not pre-irradiated) \\
\hline Peak linear power & Not applicable \\
\hline $\begin{array}{l}\text { Peak internal clad } \\
\text { temperature }\end{array}$ & Not applicable \\
\hline $\begin{array}{l}\text { Fluence } \\
\text { (spectrum) }\end{array}$ & Not applicable \\
\hline
\end{tabular}

\section{OTHER FUEL SAMPLE INFORMATION}

$173 \mathrm{~g}$ fuel per pin. $14.4 \mathrm{~cm}$ upper and lower Inconel reflectors.

\begin{tabular}{|l|l|}
\hline \multicolumn{2}{|c|}{ TEST CONDITIONS, MEASUREMENTS, \& ANALYSES } \\
\hline $\begin{array}{l}\text { Coolant } \\
\text { environment }\end{array}$ & $\begin{array}{l}320^{\circ} \mathrm{C} \text { sodium; at } 7.5 \mathrm{~m} / \mathrm{s} \text { for } 3 \mathrm{~s} \text { after reaching } \\
\text { steady power, then gradual decrease to } 2.1 \mathrm{~m} / \mathrm{s} \\
\text { during } 2.1 \mathrm{~s} \text {, then gradual decrease to } 1.5 \mathrm{~m} / \mathrm{s} \\
\text { during } 4 \mathrm{~s} \text {, then decrease to zero in } 0.5 \mathrm{~s} .\end{array}$ \\
\hline $\begin{array}{l}\text { Transient } \\
\text { conditions }\end{array}$ & $\begin{array}{l}\text { Steady reactor power initially generating 31 } \\
\text { kW/m average in test-fuel bunde }\end{array}$ \\
\hline $\begin{array}{l}\text { Fuel sample } \\
\text { configuration } \\
\text { in the test }\end{array}$ & 7-pin hexagonal bunde \\
\hline $\begin{array}{l}\text { Test vehicle \& } \\
\text { experiment } \\
\text { system }\end{array}$ & $\begin{array}{l}\text { Gas-driven, once-through loop. The hexagonal } \\
0.5 \text {-mm thick stainless steel flowtube was } \\
\text { surrounded by a molybdenum tube, with an } ~ 2.5 \\
\text { mm intermediate gas gap. }\end{array}$ \\
\hline $\begin{array}{l}\text { Measurements } \\
\text { diagnostics } \\
\text { during test }\end{array}$ & $\begin{array}{l}\text { Flowmeters, pressure transducers, } \\
\text { thermocouples, fuel motion detector } \\
\text { (hodoscope) }\end{array}$ \\
\hline $\begin{array}{l}\text { Posttest } \\
\text { coolant flow } \\
\text { condition }\end{array}$ & $\begin{array}{l}\text { Small fraction of initial sodium flow rate possible, } \\
\text { corresponding to } 99 \% \text { plugging (at the inlet). }\end{array}$ \\
\hline $\begin{array}{l}\text { Posttest } \\
\text { condition of } \\
\text { test sample(s) }\end{array}$ & $\begin{array}{l}\text { Extensive melting and relocation of cladding. } \\
\text { Little or no fuel melting. }\end{array}$ \\
\hline $\begin{array}{l}\text { Posttest } \\
\text { measurements } \\
\text { \& information }\end{array}$ & Neutron radiography, macro-examination \\
\hline $\begin{array}{l}\text { Posttest } \\
\text { analyses }\end{array}$ & $\begin{array}{l}\text { Thermal-hydraulics, cladding motion and fuel } \\
\text { motion (SAS4A) }\end{array}$ \\
\hline
\end{tabular}

\section{KEY SUMMARY INFORMATION ABOUT THE TEST}

Test R5 focused on codant and cladding behavior under conditions representative of the central region of a high power-to-flow subassembly in the FTR (FFTF) beginning of life core, to examine initial cladding motion caused by vapor streaming. The objective was to reproduce the conditions of test $\mathrm{R} 4$ but to terminate the test before fuel melting to preserve evidence of early motion of molten cladding.

Sustained channel voiding began when the coolant velocity had fallen to $1.83 \mathrm{~m} / \mathrm{s}$ and the flow decay rate was $12 \% / \mathrm{s}$. Relative to the time of boiling-induced inlet flow reversal (reference time $=0$ ), the following sequence of events occurred: initial cladding failure at $1.14 \mathrm{~s}$, upper flow-channel blockage at $2.6 \mathrm{~s}$, flowtube failure at $3.29 \mathrm{~s}$ causing a major flow perturbation due to nitrogen gas entry from outside the flowtube, reactor power termination at $4.45 \mathrm{~s}$, and lower flow-channel blockage $6.20 \mathrm{~s}$.

No significant pressure events were recorded. Posttest examination revealed that the cladding and flowtube were completely gone from the upper two-thirds of the initial test-fuel region. A thin, incomplete plug had formed in the coolant channel around the upper insulator pellets. An 8-cm-thick steel plug had formed at the bottom of the initial fuel region, which caused a nearly-complete flow blockage. The lower plug contained intact fuel pellet stacks. Above the lower plug the pellet stacks had become considerably jumbled, as also occurred at the top of the initial fuel region, but in between those locations the fused pellet stacks remained intact. There was very little centerline melting among the pellets. 


\title{
Summary of TREAT Test R-6
}

\author{
Performed 9 August 1974
}

Transient No. 1596

\section{PRE-TEST SAMPLE CHARACTERIZATION}

\begin{tabular}{|l|l|}
\hline \multicolumn{2}{|c|}{ FUEL } \\
\hline Form & He-bonded oxide \\
\hline Composition & $\begin{array}{l}\mathrm{UO}_{2} \\
\text { Outer pins: } 14 \%{ }^{235} \mathrm{U} \text { in U } \\
\text { Inner pin: } 20 \%{ }^{225} \mathrm{U} \text { in U }\end{array}$ \\
\hline Geometry & Solid pellets with dished ends \\
\hline Fissile height & $\begin{array}{l}91.44 \mathrm{~cm} \text { (prototypic of FTR power } \\
\text { fuel design) }\end{array}$ \\
\hline $\begin{array}{l}\text { Smear } \\
\text { density }\end{array}$ & $9.4 \mathrm{~g} / \mathrm{cm}^{3}$ \\
\hline
\end{tabular}

\begin{tabular}{|l|l|}
\hline \multicolumn{2}{|c|}{ CLADDING } \\
\hline Material & $316 \mathrm{SS} 20 \% \mathrm{CW}$ \\
\hline OD & $0.584 \mathrm{~cm}$ \\
\hline Thickness & $0.038 \mathrm{~cm}$ \\
\hline
\end{tabular}

\begin{tabular}{|l|l|}
\hline \multicolumn{2}{|l|}{ PRE-IRRADIATION CHARACTERISTICS } \\
\hline BU (peak axial) & (Not pre-irradiated) \\
\hline Peak linear power & Not applicable \\
\hline $\begin{array}{l}\text { Peak internal clad } \\
\text { temperature }\end{array}$ & Not applicable \\
\hline $\begin{array}{l}\text { Fluence } \\
\text { (spectrum) }\end{array}$ & Not applicable \\
\hline
\end{tabular}

\section{OTHER FUEL SAMPLE INFORMATION}

$173 \mathrm{~g}$ fuel per pin. $14.4 \mathrm{~cm}$ upper and lower Inconel reflectors.

\begin{tabular}{|l|l|}
\hline \multicolumn{2}{|c|}{ TEST CONDITIONS, MEASUREMENTS, \& ANALYSES } \\
\hline $\begin{array}{l}\text { Coolant } \\
\text { environment }\end{array}$ & $\begin{array}{l}320 \circ \text { C sodium; at } 7.4 \mathrm{~m} / \mathrm{s} \text { for } 3 \mathrm{~s} \text { after reaching } \\
\text { steady power, then linear decrease to } 3.3 \mathrm{~m} / \mathrm{s} \text { in } \\
\text { then decrease to zero in } 0.5 \mathrm{~s} \text {. (Boiling began at } \\
2 \mathrm{~m} / \mathrm{s} \text {.) }\end{array}$ \\
\hline $\begin{array}{l}\text { Transient } \\
\text { conditions }\end{array}$ & $\begin{array}{l}\text { Steady reactor power initially generating } 28 \\
\text { kW/m until scram. }\end{array}$ \\
\hline $\begin{array}{l}\text { Fuel sample } \\
\text { configuration } \\
\text { in the test }\end{array}$ & 7-pin hexagonal bunde \\
\hline $\begin{array}{l}\text { Test vehicle \& } \\
\text { experiment } \\
\text { system }\end{array}$ & $\begin{array}{l}\text { Gas-driven, once-through loop. The hexagonal } \\
\text { 0.5-mm thick stainless steel flowtube was } \\
\text { surrounded by a molybdenum tube, with an } ~ 2.5 \\
\text { mm intermediate gas gap. }\end{array}$ \\
\hline $\begin{array}{l}\text { Measurements } \\
\text { /diagnostics } \\
\text { during test }\end{array}$ & $\begin{array}{l}\text { Flowmeters, pressure transducers, } \\
\text { thermocouples, fuel motion detector } \\
\text { (hodoscope) }\end{array}$ \\
\hline $\begin{array}{l}\text { Posttest } \\
\text { coolant flow } \\
\text { condition }\end{array}$ & Complete flow blockage at the inlet. \\
\hline $\begin{array}{l}\text { Posttest } \\
\text { condition of } \\
\text { test sample(s) }\end{array}$ & $\begin{array}{l}\text { Gross meltdown of cladding and flowtube. Pell et } \\
\text { stacks of three pins still standing. }\end{array}$ \\
\hline $\begin{array}{l}\text { Posttest } \\
\text { measurements } \\
\text { \& information }\end{array}$ & Neutron radiography, macro-examination \\
\hline $\begin{array}{l}\text { Posttest } \\
\text { analyses }\end{array}$ & $\begin{array}{l}\text { Thermal-hydraulics, cladding motion and fuel } \\
\text { motion (SAS4A) }\end{array}$ \\
\hline
\end{tabular}

\section{KEY SUMMARY INFORMATION ABOUT THE TEST}

Test R6 focused on codant and cladding behavior under conditions representative of the central region of a high power-to-flow subassembly in the FTR (FFTF) beginning of life core, particularly to obtain fuel slumping information for input to planning the subsequent R7 test (which would include a power pulse consistent with reactivity addition due to fuel slumping). Test R6 reproduced test R4 as closely as possible and obtained fuel-motion data using the hodoscope.

Relative to the time of boiling-induced inlet flow reversal (reference time $=0$ ), the following sequence of events occurred: initial cladding failure at $0.90 \mathrm{~s}$, flowtube fail ure at $3.00 \mathrm{~s}$, structural melting beginning at $3.65 \mathrm{~s}$, an inlet blockage forming at $4.35 \mathrm{~s}$, downward fuel motion beginning at $5 \mathrm{~s}$ and ending at $7 \mathrm{~s}$, and the reactor scramming at $8.2 \mathrm{~s}$. No major pressure events were recorded.

Posttest examination revealed that three pins retained their column shape, although many of the pellets had become hollow. The hexagonal flowtube had melted totally along the length of the active fuel section. At the bottom of the fuel elements, cladding and spacer wires had completely melted. The steel from the flowtube and cladding, combined with some molybdenum from the wall surrounding the flowtube, had filled spaces around pellet stacks to form a massive blockage about $18 \mathrm{~cm}$ high near the original bottom of the fuel region. In the upper blanket region was a steel blockage extending across, but not totally filling, the area inside the flowtube. The top $15 \%$ of the fuel region $(x / L=0.85$ to 1.0 ) was practically empty. Elsewhere in the original fuel region the fuel was mainly a collection of short stacks of pellets or individual pellets (or pieces of pellets) in a jumble; the pellets were unmelted or partially-melted. The greatest evidence of fuel melting was among the pellets in the region $x / L=0.4$ to 0.8 . No fuel was found above or below the original ends of the fuel columns. Within the once-melted fuel, finely-dispersed metallic particles were generally found. 


\title{
Summary of TREAT Test R-7
}

\author{
Performed 27 September 1974
}

Transient No. 1602

\section{PRE-TEST SAMPLE CHARACTERIZATION}

\begin{tabular}{|l|l|}
\hline \multicolumn{2}{|c|}{ FUEL } \\
\hline Form & He-bonded oxide \\
\hline Composition & $\begin{array}{l}\mathrm{UO}_{2} \\
\text { Outer pins: } 14 \%{ }^{235} \mathrm{U} \text { in U } \\
\text { Inner pin: } 20 \%{ }^{225} \mathrm{U} \text { in U }\end{array}$ \\
\hline Geometry & Solid pellets with dished ends \\
\hline Fissile height & $\begin{array}{l}91.44 \mathrm{~cm} \text { (prototypic of FTR power } \\
\text { fuel design) }\end{array}$ \\
\hline $\begin{array}{l}\text { Smear } \\
\text { density }\end{array}$ & $9.4 \mathrm{~g} / \mathrm{cm}^{3}$ \\
\hline
\end{tabular}

\begin{tabular}{|l|l|}
\hline \multicolumn{2}{|c|}{ CLADDING } \\
\hline Material & $316 \mathrm{SS} 20 \% \mathrm{CW}$ \\
\hline OD & $0.584 \mathrm{~cm}$ \\
\hline Thickness & $0.038 \mathrm{~cm}$ \\
\hline
\end{tabular}

\begin{tabular}{|l|l|}
\hline \multicolumn{2}{|l|}{ PRE-IRRADIATION CHARACTERISTICS } \\
\hline BU (peak axial) & (Not pre-irradiated) \\
\hline Peak linear power & Not applicable \\
\hline $\begin{array}{l}\text { Peak internal clad } \\
\text { temperature }\end{array}$ & Not applicable \\
\hline $\begin{array}{l}\text { Fluence } \\
\text { (spectrum) }\end{array}$ & Not applicable \\
\hline
\end{tabular}

\section{OTHER FUEL SAMPLE INFORMATION}

$173 \mathrm{~g}$ fuel per pin. $14.4 \mathrm{~cm}$ upper and lower Inconel reflectors.

\begin{tabular}{|c|c|}
\hline \multicolumn{2}{|c|}{ TEST CONDITIONS, MEASUREMENTS, \& ANALYSES } \\
\hline $\begin{array}{l}\text { Coolant } \\
\text { environment }\end{array}$ & $\begin{array}{l}315^{\circ} \mathrm{C} \text { sodium; at constant } 7.3 \mathrm{~m} / \mathrm{s} \text { during the } \\
\text { first } 3 \mathrm{~s} \text { after reaching steady power, then flow } \\
\text { coastdown decreasing to } 40-50 \% \text { of full flow } \\
\text { within about } 2 \mathrm{~s} \text {. }\end{array}$ \\
\hline $\begin{array}{l}\text { Transient } \\
\text { conditions }\end{array}$ & $\begin{array}{l}\text { Reactor power generating } 27 \mathrm{~kW} / \mathrm{m} \text { for } 17 \mathrm{~s} \\
\text { followed by a power puls having } 0.18 \mathrm{~s} \text { full- } \\
\text { width-at-half-maximum, initiated } 6.2 \mathrm{~s} \text { after inlet } \\
\text { flow reversal, with peak } 15 \text { times the steady- } \\
\text { state power }\end{array}$ \\
\hline $\begin{array}{l}\text { Fuel sample } \\
\text { configuration } \\
\text { in the test }\end{array}$ & 7-pin hexagonal bunde \\
\hline $\begin{array}{l}\text { Test vehicle \& } \\
\text { experiment } \\
\text { system }\end{array}$ & $\begin{array}{l}\text { Gas-driven, once-through loop. The hexagonal } \\
0.5 \text {-mm thick stainless steel flowtube was } \\
\text { surrounded by a molybdenum tube, with an } \sim 2.5 \\
\text { mm intermediate gas gap. }\end{array}$ \\
\hline $\begin{array}{l}\text { Measurements } \\
\text { /diagnostics } \\
\text { during test }\end{array}$ & $\begin{array}{l}\text { Flowmeters, pressure transducers, } \\
\text { thermocouples, acoustic sensor, fuel motion } \\
\text { detector (hodoscope) }\end{array}$ \\
\hline $\begin{array}{l}\text { Posttest } \\
\text { coolant flow } \\
\text { condition }\end{array}$ & Complete flow blockage at the inlet. \\
\hline $\begin{array}{l}\text { Posttest } \\
\text { condition of } \\
\text { test sample(s) }\end{array}$ & Gross meltdown \\
\hline $\begin{array}{l}\text { Posttest } \\
\text { measurements } \\
\text { \& information }\end{array}$ & Neutron radiography, macro-examination \\
\hline $\begin{array}{l}\text { Posttest } \\
\text { analyses }\end{array}$ & $\begin{array}{l}\text { Thermal-hydraulics, cladding motion and fuel } \\
\text { motion (SAS4A) }\end{array}$ \\
\hline
\end{tabular}

\section{KEY SUMMARY INFORMATION ABOUT THE TEST}

Test R7 simulated an unprotected loss-of-flow situation in the central channels of a high power-to-flow subassembly in an FTR (FFTF)like reactor including a power excursion due to reactivity feedback associated with fuel slumping. Conditions for motion of molten core materials driven by fuel and steel vapor pressure within a region plugged at top and bottom by steel blockages were predicted. The power pulse was timed to coincide with the onset of fuel slumping using analytical predictions and results from test R6. The R7 apparatus was nearly identical to that of test R6, but (unlike in the previous R-series tests) the annular gas space outside the flowtube was filled at a reduced pressure so that when the flowtube began melting, the gas pressure would approximately equal the sodium channel pressure. The hodoscope viewed only the axial-center half of the original fuel region $(x / L=0.25$ to 0.75$)$.

Relative to the time of boiling-induced inlet flow reversal (reference time $=0$ ), the following sequence of events occurred: initial cladding failure at $1.24 \mathrm{~s}$, upper flow channel blockage at $2.8 \mathrm{~s}$, flowtube failure at $3.72 \mathrm{~s}$, melted structural material beginning to move at $4.8 \mathrm{~s}$, a significant amount of fuel having moved radially beyond the flowtube by $5.5 \mathrm{~s}$, the power spike beginning at $6.2 \mathrm{~s}, \sim 40 \%$ of the fuel initially in the region $x / L=0.25$ to $0.75(250 \mathrm{~g})$ moving axially out of that region, an inlet blockage forming at $6.6 \mathrm{~s}, \sim 170 \mathrm{~g}$ of fuel returning to the region $x / L=0.25$ to 0.75 , the reactor scramming at $7.7 \mathrm{~s}$, and then $\sim 120 \mathrm{~g}$ of fuel again leaving the region $x / L=0.25$ to 0.75 . No major pressure events were recorded.

Posttest examination revealed that claddings, spacer wires, and the flowtube were completely melted from $x / L=0$ to 1.0 . Fuel pellets filled the inside of the Mo tube in a disarray from $x / L=0$ to 0.06 and in irregular stacks from $x / L=0.06$ to 0.12 . Melted steel (but no melted fuel) completely filled the spaces between these pellets radially outward to the Mo wall. A dense mass of fuel, most of which had previously been melted, filled the region $x / L=0.13$ to 0.25 . Above it, from $x / L=0.25$ to 0.5 , was a less-dense, sponge-like mass. From $x / L=0.5$ to 0.6 was a dense fuel mass that occupied much of the area within the Mo tube. On top of that was a more-dense fuel accumulation. There was another accumulation of fuel at the top of the original fuel region $(x / L=0.97-1.05)$. An incomplete flow blockage was present at the top of the original fuel column region. 


\section{Summary of TREAT Test R-8

\author{
Performed 7 May 1976
} \\ Transient No. 1780}

\section{PRE-TEST SAMPLE CHARACTERIZATION}

\begin{tabular}{|l|l|}
\hline \multicolumn{2}{|c|}{ FUEL } \\
\hline Form & He-bonded oxide \\
\hline Composition & $\begin{array}{l}\mathrm{UO}_{2} \\
\text { Outer pins: } 14 \%{ }^{235} \mathrm{U} \text { in U } \\
\text { Inner pin: } 20 \%{ }^{225} \mathrm{U} \text { in U }\end{array}$ \\
\hline Geometry & Solid pellet w/dished ends \\
\hline Fissile height & $\begin{array}{l}91.44 \mathrm{~cm} \text { (prototypic of FTR power } \\
\text { fuel design) }\end{array}$ \\
\hline $\begin{array}{l}\text { Smear } \\
\text { density }\end{array}$ & $9.4 \mathrm{~g} / \mathrm{cm}^{3}$ \\
\hline
\end{tabular}

\begin{tabular}{|l|l|}
\hline \multicolumn{2}{|c|}{ CLADDING } \\
\hline Material & $316 \mathrm{SS} 20 \% \mathrm{CW}$ \\
\hline OD & $0.584 \mathrm{~cm}$ \\
\hline Thickness & $0.038 \mathrm{~cm}$ \\
\hline
\end{tabular}

\begin{tabular}{|l|l|}
\hline PRE-IRRADIATION CHARACTERISTICS \\
\hline BU (peak axial) & (Not pre-irradiated) \\
\hline Peak linear power & Not applicable \\
\hline $\begin{array}{l}\text { Peak internal clad } \\
\text { temperature }\end{array}$ & Not applicable \\
\hline $\begin{array}{l}\text { Fluence } \\
\text { (spectrum) }\end{array}$ & Not applicable \\
\hline
\end{tabular}

\section{OTHER FUEL SAMPLE INFORMATION}

Three pins were pressurized with Xe to nominal end-oflife pressure (1.4 MPa). Four pins had beginning-of-life pressure. $173 \mathrm{~g}$ fuel per pin. $14.4 \mathrm{~cm}$ upper and lower Inconel reflectors.

\begin{tabular}{|l|l|}
\hline \multicolumn{2}{|c|}{ TEST CONDITIONS, MEASUREMENTS, \& ANALYSES } \\
\hline $\begin{array}{l}\text { Coolant } \\
\text { environment }\end{array}$ & $\begin{array}{l}315^{\circ} \mathrm{C} \text { sodium; at constant 7.15 m/s during the } \\
\text { first 3 s after reaching steady power, then flow } \\
\text { coastdown. }\end{array}$ \\
\hline $\begin{array}{l}\text { Transient } \\
\text { conditions }\end{array}$ & $\begin{array}{l}\text { Steady reactor power initially producing } 31.8 \\
\text { kW/m in the test fuel. }\end{array}$ \\
\hline $\begin{array}{l}\text { Fuel sample } \\
\text { configuration } \\
\text { in the test }\end{array}$ & 7-pin hexagonal bundle \\
\hline $\begin{array}{l}\text { Test vehicle \& } \\
\text { experiment } \\
\text { system }\end{array}$ & $\begin{array}{l}\text { Gas-driven, once-through loop. The hexagonal } \\
\text { surrounded by a molybdenum tube, with an } ~ 2.5 \\
\text { mm intermediate gas gap. }\end{array}$ \\
\hline $\begin{array}{l}\text { Measurements } \\
\text { /diagnostics } \\
\text { during test }\end{array}$ & $\begin{array}{l}\text { Flowmeters, pressure transducers, } \\
\text { thermocouples, acoustic sensor, fuel motion } \\
\text { detector (hodoscope) }\end{array}$ \\
\hline $\begin{array}{l}\text { Posttest } \\
\text { coolant flow } \\
\text { condition }\end{array}$ & Complete flow blockage at the inlet. \\
\hline $\begin{array}{l}\text { Posttest } \\
\text { condition of } \\
\text { test sample(s) }\end{array}$ & $\begin{array}{l}\text { Gross meltdown (except for a length of partially- } \\
\text { intact cladding expelled from the fuel region) }\end{array}$ \\
\hline $\begin{array}{l}\text { Posttest } \\
\text { measurements } \\
\text { \& information }\end{array}$ & Neutron radiography, macro-examination \\
\hline $\begin{array}{l}\text { Posttest } \\
\text { analyses }\end{array}$ & $\begin{array}{l}\text { Thermal-hydraulics, cladding motion and fuel } \\
\text { motion (SAS4A) }\end{array}$ \\
\hline
\end{tabular}

KEY SUMMARY INFORMATION ABOUT THE TEST

Test R8 investigated the effect on the loss-of-flow sequence of the release of pressurized non-condensable plenum gas into the coolant channel. Sodium voiding began $9.5 \mathrm{~s}$ into the coastdown when the coolant velocity had decreased by $71 \%$. The inlet flow reversed $0.7 \mathrm{~s}$ later. Relative to the time of flow reversal (reference time $=0$ ), the following sequence occurred: cladding fail ures began at $0.71 \mathrm{~s}$ (above the midplane, with the three pressurized pins failing within $20 \mathrm{~ms}$ ), the flowtube breached at $0.78 \mathrm{~s}$ (and $2 \%$ of the fuel moved above the original fuel region), sodium was expelled from the fued region from 1.5 to $2.2 \mathrm{~s}$, there was single attempt by the inlet sodium to re-enter the fuel region, an inlet blockage formed at $2.5 \mathrm{~s}$, and downward fuel motion began at $3.8 \mathrm{~s}$ and continued until scram at $7.8 \mathrm{~s}$. The downward fuel motion was into the lower third of the original fuel region and radially beyond the flowtube to the surrounding Mo tube.

Posttest examination showed there was no steel blockage in the upper insulator and reflector region, although much of the original steel there had melted. There was essentially no fuel above the original fuel region. The top parts of two of the pressurized pins had been driven upward $6 \mathrm{~cm}$ and $10 \mathrm{~cm}$, limited by a mechanical restraint. The other pressurized pin had been mechanically severed $9 \mathrm{~cm}$ below the top of the original fuel column, and the long cladding segment above the severed location had been driven $74 \mathrm{~cm}$ upward, without restraint. The bottom $5 \mathrm{~cm}$ of the latter cladding segment showed an axial rip, apparenty indicating the location and mode of initial cladding breach. The long cladding segment appeared to have slid off of the top pellets and reflector rod during its ejection.

The entire upper quarter of the original fuel region $(x / L=0.75$ to 1.0$)$ had slumped down, and fuel had significanty penetrated into the region outside of the flowtube. The top $30 \%$ of the flowtube along the fuel column ( $x / L=0.7$ to 1.0 ) had also melted away, some of it apparenty moving downward along the inner surface of the molybdenum tube. Practically no fuel remained in the fuel region $x / L=0.6$ to 1.0). Some fuel remained in the region $x / L=0.1$ to 0.6 , but from $x / L=-0.1$ to 0.1 there was a dense accumulation of fuel on top of steel extending radially to the Mo tube. 


\section{Summary of TREAT Test R-9

\author{
Performed 9 April 1976
}

Transient No. 1776

\section{PRE-TEST SAMPLE CHARACTERIZATION}

\begin{tabular}{|l|l|}
\hline \multicolumn{2}{|c|}{ FUEL } \\
\hline Form & He-bonded oxide \\
\hline Composition & $\begin{array}{l}\mathrm{UO}_{2} \\
\text { Outer pins: } 14 \%{ }^{235} \mathrm{U} \text { in U } \\
\text { Inner pin: } 20 \%{ }^{235} \mathrm{U} \text { in U }\end{array}$ \\
\hline Geometry & Solid pellet with dished ends \\
\hline Fissile height & $91.44 \mathrm{~cm}$ (prototypic of FFTF/FTR) \\
\hline $\begin{array}{l}\text { Smear } \\
\text { density }\end{array}$ & $\begin{array}{l}\text { Outer pins: } 9.40 \mathrm{~g} / \mathrm{cm}^{3} \\
\text { Inner pin: } 9.39 \mathrm{~g} / \mathrm{cm}^{3}\end{array}$ \\
\hline
\end{tabular}

\begin{tabular}{|l|l|}
\hline \multicolumn{2}{|c|}{ CLADDING } \\
\hline Material & $316 \mathrm{SS} \mathrm{20 \%} \mathrm{CW}$ \\
\hline OD & $0.584 \mathrm{~cm}$ \\
\hline Thickness & $0.038 \mathrm{~cm}$ \\
\hline
\end{tabular}

PRE-IRRADIATION CHARACTERISTICS

\begin{tabular}{|l|l|}
\hline BU (peak axial) & (Not preirradiated) \\
\hline Peak linear power & Not applicable \\
\hline $\begin{array}{l}\text { Peak internal clad } \\
\text { temperature }\end{array}$ & Not applicable \\
\hline $\begin{array}{l}\text { Fluence } \\
\text { (spectrum) }\end{array}$ & Not applicable \\
\hline
\end{tabular}

\section{OTHER FUEL SAMPLE INFORMATION}

$173 \mathrm{~g}$ fuel per pin; $14.4 \mathrm{~cm}$ long upper and lower Inconel reflectors; $8.67 \mathrm{~cm}^{3}$ plenum volume, sealed at $1 \mathrm{~atm}$ at $24^{\circ} \mathrm{C}$

\begin{tabular}{|c|c|}
\hline \multicolumn{2}{|c|}{ TEST CONDITIONS, MEASUREMENTS, \& ANALYSES } \\
\hline $\begin{array}{l}\text { Coolant } \\
\text { environment }\end{array}$ & $\begin{array}{l}\text { Flowing sodium at } 7.6 \mathrm{~m} / \mathrm{s}, 371^{\circ} \mathrm{C} \text { inlet } \\
\text { temperature }\end{array}$ \\
\hline $\begin{array}{l}\text { Transient } \\
\text { conditions }\end{array}$ & $\begin{array}{l}5.2-\mathrm{s} \text { flattop preheat at } 33 \mathrm{~kW} / \mathrm{m} \text { (bundle axial } \\
\text { average), then } 50 \mathrm{~d} / \mathrm{s} \text { TOP burst }(\sim 1.5 \text {-s e-fold } \\
\text { period). Reactor scrammed } 3.5 \text { s into burst. }\end{array}$ \\
\hline $\begin{array}{l}\text { Fuel sample } \\
\text { configuration } \\
\text { in the test }\end{array}$ & 7-pin hexagonal bunde \\
\hline $\begin{array}{l}\text { Test vehicle \& } \\
\text { experiment } \\
\text { system }\end{array}$ & $\begin{array}{l}\text { Gas-driven once-through sodium loop. A } 0.5 \text { - } \\
\text { mm-thick hexagonal stainless steel flowtube } \\
\text { surrounded the fuel pin bundle. }\end{array}$ \\
\hline $\begin{array}{l}\text { Measurements } \\
\text { /diagnostics } \\
\text { during test }\end{array}$ & $\begin{array}{l}\text { Flowmeters, pressure transducers, } \\
\text { thermocouples, acoustic microphone, flux } \\
\text { monitor wires, fuel motion detector (hodoscope) }\end{array}$ \\
\hline $\begin{array}{l}\text { Posttest } \\
\text { coolant flow } \\
\text { condition }\end{array}$ & No flow was possible. \\
\hline $\begin{array}{l}\text { Posttest } \\
\text { condition of } \\
\text { test sample(s) }\end{array}$ & $\begin{array}{l}\text { Gross melting and extensive redistribution of fuel } \\
\text { and cladding, with about half of the fuel } \\
\text { relocated downstream of the initial fuel region. } \\
\text { Complete upper flow blockage and possibly } \\
\text { complete lower flow blockage. }\end{array}$ \\
\hline $\begin{array}{l}\text { Posttest } \\
\text { measurements } \\
\& \text { information }\end{array}$ & $\begin{array}{l}\text { Gamma, X-ray, and neutron radiography; macro- } \\
\text { examinations, X-ray spectroscopy }\end{array}$ \\
\hline $\begin{array}{l}\text { Posttest } \\
\text { analyses }\end{array}$ & $\begin{array}{l}\text { Fuel and cladding motion (SAS4A), thermal- } \\
\text { hydraulics }\end{array}$ \\
\hline
\end{tabular}

\section{KEY SUMMARY INFORMATION ABOUT THE TEST}

Test R-9 investigated the codant, cladding, and fued dynamics resulting from an FFTF $50 \mathrm{~d} / \mathrm{s}$ transient overpower accident on beginningof-life full-length fuel and provided data for comparison with SAS computations. Codant boiling was detected $2.58 \mathrm{~s}$ into the overpower ramp, and initial pin failure occurred 0.14 s later, when the bundle-averaged fuel power was $150 \mathrm{~kW} / \mathrm{m}$. The initial pin fail ure did not cause an inlet flow reversal or high-pressure pulses, but there was significant voiding of the test-fuel region, and the coolant flowtube failed (creating a path for materials to move into the annular space outside the flowtube). The inlet flow briefly reversed at $0.05 \mathrm{~s}, 0.14 \mathrm{~s}$, and $0.67 \mathrm{~s}$ after initial pin failure. Fuel motion began $0.07 \mathrm{~s}$ after the first reversal, with upward fuel motion into the fuel pin plenum region (which continued during the next $0.45 \mathrm{~s}$ ). At the second inlet sodium flow reversal, most of the fuel region was voided of sodium, although some sodium may have flowed into the annulus outside the breached flowtube. Beginning $0.09 \mathrm{~s}$ before the third flow reversal, much more fuel began moving to and above the top of the original fuel zone and continued to do so for the next $0.40 \mathrm{~s}$. Downward fuel motion began $0.06 \mathrm{~s}$ after the third inlet sodium flow reversal, with fuel moving below the original fuel region. The reactor scrammed $1.02 \mathrm{~s}$ after initial fuel pin failure, followed $0.5 \mathrm{~s}$ later by a significant (but not very energetic) sodium flow event. Inlet codant flow oscillations of decreasing magnitude occurred as the sodium repeatedly attempted to reenter the hot voided fuel zone. Misalignment of the hodoscope collimator prevented quantitative mass analysis of the fuel-motion data. Posttest examination showed that over $90 \%$ of the test fuel had melted, the flowtube had melted over the upper $75 \%$ of the original fuel region (and above) and had been redistributed, and about $53 \%$ of the fuel had been swept downstream above the active fuel zone, The upper parts of three of the pins had been pushed 23 to $42 \mathrm{~cm}$ above their initial locations, and the upper axial reflectors had completely melted. The coolant channel had become totally blocked by a $27-\mathrm{cm}$-high accumulation of fuel and steel centered at an elevation $47 \mathrm{~cm}$ above the original fuel region. Some material had relocated as high as one meter above the original fuel region. The lower reflector region had become considerably blocked by a $4.5-\mathrm{cm}$ high accumulation of fuel and possibly once-molten metal. 


\title{
Summary of TREAT Test R-12
}

\author{
Performed 23 June 1977
}

Transient No. 1915

\begin{tabular}{l}
\hline \multicolumn{2}{|l|}{ PRE-TEST SAMPLE CHARACTERIZATION } \\
\begin{tabular}{|l|l|}
\hline \multicolumn{2}{|c|}{ FUEL } \\
\hline Form & He-bonded oxide \\
\hline Composition & $\begin{array}{l}\mathrm{UO}_{2} \\
\text { Outer pins: } 14 \% 235 \mathrm{U} \text { in U } \\
\text { Inner pin: } 20 \% 235 \mathrm{U} \text { in U }\end{array}$ \\
\hline Geometry & Solid pellet with dished ends \\
\hline Fissile height & $\begin{array}{l}91.44 \mathrm{~cm} \text { (prototypic of } \\
\text { FFTF/FTR design) }\end{array}$ \\
\hline $\begin{array}{l}\text { Smear } \\
\text { density }\end{array}$ & $\begin{array}{l}\text { Outer pins: } 9.40 \mathrm{~g} / \mathrm{cm}^{3} \\
\text { Inner pin: } 9.39 \mathrm{~g} / \mathrm{cm}^{3}\end{array}$ \\
\hline
\end{tabular}
\end{tabular}

\begin{tabular}{|l|l|}
\hline \multicolumn{2}{|c|}{ CLADDING } \\
\hline Material & $316 \mathrm{SS} 20 \% \mathrm{CW}$ \\
\hline OD & $0.584 \mathrm{~cm}$ \\
\hline Thickness & $0.038 \mathrm{~cm}$ \\
\hline
\end{tabular}

\begin{tabular}{|c|c|}
\hline \multicolumn{2}{|c|}{ PRE-IRRADIATION CHARACTERISTICS } \\
\hline BU & (Not pre-irradiated) \\
\hline Peak linear power & Not applicable \\
\hline $\begin{array}{l}\text { Peak internal clad } \\
\text { temperature }\end{array}$ & Not applicable \\
\hline Fluence & Not applicable \\
\hline \multicolumn{2}{|c|}{ OTHER FUEL SAMPLE INFORMATION } \\
\hline \multicolumn{2}{|c|}{$\begin{array}{l}173 \mathrm{~g} \text { fued per pin; } 14.4 \mathrm{~cm} \text { long upper and lower Incone } \\
\text { reflectors; } 8.67 \mathrm{~cm}^{3} \text { plenum, sealed at } 1 \mathrm{~atm} \text { at } 24{ }^{\circ} \mathrm{C} \text {. }\end{array}$} \\
\hline
\end{tabular}

\begin{tabular}{|c|c|}
\hline \multicolumn{2}{|c|}{ TEST CONDITIONS, MEASUREMENTS, \& ANALYSES } \\
\hline $\begin{array}{l}\text { Coolant } \\
\text { environment }\end{array}$ & $\begin{array}{l}\text { Flowing sodium at } 7.6 \mathrm{~m} / \mathrm{s}, 371^{\circ} \mathrm{C} \text { inlet } \\
\text { temperature }\end{array}$ \\
\hline $\begin{array}{l}\text { Transient } \\
\text { conditions }\end{array}$ & $\begin{array}{l}\text { 5-s preheat at } 34 \mathrm{~kW} / \mathrm{m} \text { (bundle axial average), } \\
\text { then } 50 \mathrm{c} / \mathrm{s} \text { TOP burst ( 1.6-s e-fold period). } \\
\text { Reactor scrammed } 2.8 \mathrm{~s} \text { into burst (at } 170 \mathrm{~kW} / \mathrm{m} \\
\text { average) due to inlet flowrate drop. }\end{array}$ \\
\hline $\begin{array}{l}\text { Fuel sample } \\
\text { configuration } \\
\text { in the test }\end{array}$ & 7-pin hexagonal bundle \\
\hline $\begin{array}{l}\text { Test vehicle \& } \\
\text { experiment } \\
\text { system }\end{array}$ & $\begin{array}{l}\text { Gas-driven once-through sodium loop. A } 0.5- \\
\text { mm-thick hexagonal stainless steel flowtube } \\
\text { surrounded the fuel pin bunde. }\end{array}$ \\
\hline $\begin{array}{l}\text { Measurements } \\
\text { /diagnostics } \\
\text { during test }\end{array}$ & $\begin{array}{l}\text { Flowmeters, pressure transducers, } \\
\text { thermocouples, acoustic microphone, flux } \\
\text { monitor wires, fuel motion detector (hodoscope) }\end{array}$ \\
\hline $\begin{array}{l}\text { Posttest } \\
\text { coolant flow } \\
\text { condition }\end{array}$ & Same as initial flow rate. \\
\hline $\begin{array}{l}\text { Posttest } \\
\text { condition of } \\
\text { test sample(s) }\end{array}$ & $\begin{array}{l}\text { Failure of all seven pins. } 10 \% \text { of fuel escaped } \\
\text { from cladding, of which half relocated to above } \\
\text { the initial fuel region. The flowtube also failed. }\end{array}$ \\
\hline $\begin{array}{l}\text { Posttest } \\
\text { measurements } \\
\text { \& information }\end{array}$ & $\begin{array}{l}\text { Gamma, X-ray, and neutron radiography; macro- } \\
\text { examinations, X-ray spectroscopy }\end{array}$ \\
\hline $\begin{array}{l}\text { Posttest } \\
\text { analyses }\end{array}$ & $\begin{array}{l}\text { Fuel and cladding motion (SAS4A), thermal- } \\
\text { hydraulics }\end{array}$ \\
\hline
\end{tabular}

\section{KEY SUMMARY INFORMATION ABOUT THE TEST}

An objective of test R12 was to investigate post-failure fuel coolability in an FFTF 50 c/s transient overpower accident simulation with reactor scram occurring immediately upon initial disruptive pin failure. Test R12 was to be compared to test R9, in which the power transient was identical to the one in R-12 but continued long after pin failure. As in test R9, a steel heat sink adjacent to the bottom of the fuel caused $25 \%$ lower power along the bottom third of the test fuel than along the top third. The power burst in R12 was automatically terminated when the inlet flow rate dropped by $60 \%$, which occurred $2.82 \mathrm{~s}$ into the burst when the major coolant dynamics event of the test occurred (at a reactor energy within $5 \%$ of the energy at initial pin failure in test R9). However, failure of one or more fuel pins had occurred when only two-thirds of the reactor burst energy had been generated, accompanied by minor coolant perturbations, flowtube breach, and $2 \%$ of the fuel relocating above the fuel columns. During the event causing scram, expelled fuel moved back into the fuelcolumn region. After scram, $6 \%$ of the original fuel mass moved from the bottom half of the original fuel zone to above the midplane. Full coolant flow was reestablished posttest.

Posttest examination showed that all seven pins failed in a highly-localized region at the top of the active fuel zone, the hexagonal flowtube had been breached at that location, about $5 \%$ of the fuel inventory had been swept downstream out of the active fuel zone, and $90 \%$ of the fuel remained within the cladding tubes. The breaches of five of the peripheral pins faced the central pin; the breach of the other peripheral pin faced the flowtube breach. The breaches were due mainly to cladding melting; there was no cladding diameter increase anywhere along the fuel columns. Some molten fuel evidenty flowed downward within the cladding. By the end of the test, the central pin had lost about $13 \%$ of its fuel, and the peripheral pins had each lost $5-20 \%$. The fuel axial distribution was roughly uniform except sharply lower at the fuel top, and the magnitude of the loss varied linearly with the measured fuel peak-axial areal melt fraction, which varied from $55-88 \%$. The plenum springs had compressed $7-22 \mathrm{~mm}$ among the test pins due to the transient. 


\section{Appendix M: One-page Summaries of E-series Tests}

One-page summaries of each of the E-series tests are included here. These summaries can also be found in TREXR [6]. 


\title{
Summary of TREAT Test E1
}

\author{
Performed 2 July 1969
}

Transient No. 1281

\section{PRE-TEST SAMPLE CHARACTERIZATION}

\begin{tabular}{|l|l|}
\hline \multicolumn{2}{|c|}{ FUEL } \\
\hline Form & Uranium oxide \\
\hline Composition & $\mathrm{UO}_{2}, 13 \%$ enriched $\mathrm{U}$ \\
\hline Geometry & Clad pellet stack \\
\hline Fissile height & $\sim 305 \mathrm{~mm}$ \\
\hline $\begin{array}{l}\text { Smear } \\
\text { density }\end{array}$ & $9.9 \mathrm{~g} / \mathrm{cm}^{3}(90 \%$ of theoretical) \\
\hline
\end{tabular}

\begin{tabular}{|l|l|}
\hline \multicolumn{2}{|c|}{ CLADDING } \\
\hline Material & Type 304 L stainless steel, annealed \\
\hline OD & $7.37 \mathrm{~cm}$ \\
\hline Thickness & $0.51 \mathrm{~mm}$ \\
\hline
\end{tabular}

\begin{tabular}{|l|l|}
\hline \multicolumn{2}{|l|}{ PRE-IRRADIATION CHARACTERISTICS } \\
\hline BU (peak axial) & Zero \\
\hline Peak linear power & (not applicable) \\
\hline $\begin{array}{l}\text { Peak internal clad } \\
\text { temperature }\end{array}$ & (not applicable) \\
\hline $\begin{array}{l}\text { Fluence } \\
\text { (spectrum) }\end{array}$ & (not applicable) \\
\hline
\end{tabular}

\section{OTHER FUEL SAMPLE INFORMATION}

There was only a stainless-sted coil spring on top of the $\mathrm{UO}_{2}$ fuel-pellet stack.

\begin{tabular}{|l|l|}
\hline \multicolumn{2}{|c|}{ TEST CONDITIONS, MEASUREMENTS, \& ANALYSES } \\
\hline $\begin{array}{l}\text { Coolant } \\
\text { environment }\end{array}$ & $\begin{array}{l}\text { Sodium flow at } 3.8 \mathrm{~m} / \mathrm{s}(\sim 1100 \mathrm{~cm} 3 / \mathrm{s}) \text { and } 410 \\
{ }^{\circ} \mathrm{C}\end{array}$ \\
\hline $\begin{array}{l}\text { Transient } \\
\text { conditions }\end{array}$ & $\begin{array}{l}\text { A } 0.27 \text { s full-width-at-half-maximum power burst } \\
\text { driven by a reactivity step, generating } \sim 1.7 \mathrm{~kJ} / \mathrm{g} \\
\text { in the test fuel, and } ~ 1900 \mathrm{~kW} / \mathrm{m} \text { at peak power }\end{array}$ \\
\hline $\begin{array}{l}\text { Fuel sample } \\
\text { configuration } \\
\text { in the test }\end{array}$ & $\begin{array}{l}\text { Single cladded fuel pin surrounded by six } \\
\text { helium-filled hollow cladding tubes ("dummy } \\
\text { pins"); all cladding wire-wrapped }\end{array}$ \\
\hline $\begin{array}{l}\text { Test vehicle \& } \\
\text { experiment } \\
\text { system }\end{array}$ & Mark-II integral sodium loop \\
\hline $\begin{array}{l}\text { Measurements } \\
\text { /diagnostics } \\
\text { during test }\end{array}$ & $\begin{array}{l}\text { Temperature, flow rates, pressures, fuel motion } \\
\text { (hodoscope) }\end{array}$ \\
\hline $\begin{array}{l}\text { Posttest } \\
\text { coolant flow } \\
\text { condition }\end{array}$ & Same as initial flow rate \\
\hline $\begin{array}{l}\text { Posttest } \\
\text { condition of } \\
\text { test sample(s) }\end{array}$ & $\begin{array}{l}\text { No cladding failure but extensive axial in-pin fuel } \\
\text { relocation. }\end{array}$ \\
\hline $\begin{array}{l}\text { Posttest } \\
\text { measurements } \\
\text { \& information }\end{array}$ & $\begin{array}{l}\text { X-radiography, neutron radiography, macro- } \\
\text { photography, micro-photography, cladding } \\
\text { diameter measurements }\end{array}$ \\
\hline $\begin{array}{l}\text { Posttest } \\
\text { analyses }\end{array}$ & Thermal-hydraulics, cladding mechanics \\
\hline
\end{tabular}

\section{KEY SUMMARY INFORMATION ABOUT THE TEST}

Test $\mathrm{E} 1$ was the first in a series of meltdown tests on stainless-steel-clad oxide fuel in a flowing-sodium environment allowing coolant expulsion and reentry. The total test-fuel energy generation was chosen to be similar to that in stagnant-sodium TREAT test S-3. Gross in-pin axial fuel motion began late in the transient when the power had dropped to about half its peak and $\sim 1.6 \mathrm{~kJ} / \mathrm{g}$ had been generated in the test fuel. The cladding remained intact despite experiencing a strain of $\sim 1.1 \%$ along the original fuel-column region, the latter being much larger than could have been due to hoop stress caused by gas pressure. Failure was probably prevented by the axial fuel motion. The fuel had moved upward within the cladding, some reaching the top of the plenum, resulting in about a dozen large voids, roughly spaced axially about every two to three $\mathrm{cm}$ along nearly the entire length of the pin. The voids, apparently formed by large gas bubbles, were rounded at the top of the void and tapered at the bottom. The maximum areal melt fraction of the fuel was $\sim 80 \%$, with an outer rim remaining unmelted, although some melted fuel penetrated cracks in the rim and contacted the cladding. 


\title{
Summary of TREAT Test E2
}

\author{
Performed 4 September 1969
}

Transient No. 1283

\section{PRE-TEST SAMPLE CHARACTERIZATION}

\begin{tabular}{|l|l|}
\hline \multicolumn{2}{|c|}{ FUEL } \\
\hline Form & Uranium oxide \\
\hline Composition & $\mathrm{UO}_{2}, 13 \%$ enriched $\mathrm{U}$ \\
\hline Geometry & Clad pellet stack \\
\hline Fissile height & $\sim 305 \mathrm{~mm}$ \\
\hline $\begin{array}{l}\text { Smear } \\
\text { density }\end{array}$ & $9.9 \mathrm{~g} / \mathrm{cm}^{3}(90 \%$ of theoretical) \\
\hline
\end{tabular}

\begin{tabular}{|l|l|}
\hline \multicolumn{2}{|c|}{ CLADDING } \\
\hline Material & Type 304 L stainless steel, annealed \\
\hline OD & $7.37 \mathrm{~mm}$ \\
\hline Thickness & $0.51 \mathrm{~mm}$ \\
\hline
\end{tabular}

\begin{tabular}{|l|l|}
\hline \multicolumn{2}{|l|}{ PRE-IRRADIATION CHARACTERISTICS } \\
\hline BU (peak axial) & Zero \\
\hline Peak linear power & (not applicable) \\
\hline $\begin{array}{l}\text { Peak internal clad } \\
\text { temperature }\end{array}$ & (not applicable) \\
\hline $\begin{array}{l}\text { Fluence } \\
\text { (spectrum) }\end{array}$ & (not applicable) \\
\hline
\end{tabular}

\section{OTHER FUEL SAMPLE INFORMATION}

On top of the $\mathrm{UO}_{2}$ fuel pellet stack was a depleted 1.3cm-long $\mathrm{UO}_{2}$ fuel pellet; then a 5-cm long, 12-g,

stainless steel rod.

\begin{tabular}{|l|l|}
\hline \multicolumn{2}{|c|}{ TEST CONDITIONS, MEASUREMENTS, \& ANALYSES } \\
\hline $\begin{array}{l}\text { Coolant } \\
\text { environment }\end{array}$ & $\begin{array}{l}\text { Sodium flow at } 4.0 \mathrm{~m} / \mathrm{s}(\sim 1100 \mathrm{~cm} / \mathrm{s}) \text { and } 400 \\
\text { 'C }\end{array}$ \\
\hline $\begin{array}{l}\text { Transient } \\
\text { conditions }\end{array}$ & $\begin{array}{l}\text { A 0.24 s full-width-at-half-maximum power burst } \\
\text { driven by a reactivity step, reaching } 3400 \mathrm{~kW} / \mathrm{m} \\
\text { peak linear power in the test fuel and generating } \\
\sim 2.7 \mathrm{~kJ} / \mathrm{g} .\end{array}$ \\
\hline $\begin{array}{l}\text { Fuel sample } \\
\text { configuration } \\
\text { in the test }\end{array}$ & $\begin{array}{l}\text { Single cladded fued pin surrounded by six } \\
\text { helium-filled hollow cladding tubes ("dummy } \\
\text { pins"); all cladding wire-wrapped }\end{array}$ \\
\hline $\begin{array}{l}\text { Test vehicle \& } \\
\text { experiment } \\
\text { system }\end{array}$ & Mark-II integral sodium loop \\
\hline $\begin{array}{l}\text { Measurements } \\
\text { /diagnostics } \\
\text { during test }\end{array}$ & $\begin{array}{l}\text { Temperature, flow rates, pressures, fuel motion } \\
\text { (hodoscope) }\end{array}$ \\
\hline $\begin{array}{l}\text { Posttest } \\
\text { coolant flow } \\
\text { condition }\end{array}$ & Nearly complete flow blockage \\
\hline $\begin{array}{l}\text { Posttest } \\
\text { condition of } \\
\text { test sample(s) }\end{array}$ & $\begin{array}{l}\text { Cadding failure; fuel extremely fragmented and } \\
\text { extensively dispersed }\end{array}$ \\
\hline $\begin{array}{l}\text { Posttest } \\
\text { measurements } \\
\text { \& information }\end{array}$ & $\begin{array}{l}\text { X-radiography, macro-photography, micro- } \\
\text { photography, cladding diameter measurements, } \\
\text { electron microprobe analysis }\end{array}$ \\
\hline $\begin{array}{l}\text { Posttest } \\
\text { analyses }\end{array}$ & Thermal-hydraulics, cladding mechanics \\
\hline
\end{tabular}

\footnotetext{
KEY SUMMARY INFORMATION ABOUT THE TEST

Test E2 was the first test in TREAT on the meltdown of stainless-steel-clad oxide fuel in a flowing-sodium environment allowing codant expulsion and reentry. Gadding failure occurred late in the transient when the power had dropped to about half its peak and about 1.8 $\mathrm{kJ} / \mathrm{g}$ had been generated in the test fuel. Extensive failure of the central pin cladding occurred along the top $4 \mathrm{~cm}$ of the fuel stack. To a lesser extent, the cladding also melted through along a spiral path following the downstream side of the spiral spacer wire. The maximum calculated areal fuel melting before failure was $90 \%$. The fuel was extremely fragmented and dispersed, with packed fines nearly completely blocking the flow channels. The insulator pellet and stainless steel rod had moved upward about $8 \mathrm{~mm}$. There was no fuel left in the cladding except for $\sim 6 \%$ in the form of a $0.3 \mathrm{~mm}$ thick apparently unmelted circumferential shell of fuel against the bottom third of the cladding inner surface. Nearly all of the expelled fuel had become finely divided by the end of the test. The fines had become packed among the cladding tubes. The central pin cladding strain was $1.5 \%$ along the original length of the fuel column. There was no evidence of chemical reaction between fuel and cladding. The six dummy tubes were melted through on the sides facing adjacent regions of massive failures of the central pin. Conversion of fuel energy to work on the codant was $\sim 0.06 \%$.
} 


\section{Summary of TREAT Test E3}

Performed 19 June 1970

Transient No. 1322

\section{PRE-TEST SAMPLE CHARACTERIZATION}

\begin{tabular}{|l|l|}
\hline \multicolumn{2}{|c|}{ FUEL } \\
\hline Form & Uranium oxide \\
\hline Composition & $\mathrm{UO}_{2}, 13.3 \%$ enriched U. \\
\hline Geometry & Pellet stack in cladding \\
\hline Fissile height & $145 \mathrm{~mm}$ \\
\hline $\begin{array}{l}\text { Smear } \\
\text { density }\end{array}$ & $\sim 9.2 \mathrm{~g} / \mathrm{cm}^{3}$ \\
\hline
\end{tabular}

\begin{tabular}{|l|l|}
\hline \multicolumn{2}{|c|}{ CLADDING } \\
\hline Material & Type 304 L stainless steel (annealed) \\
\hline OD & $4.42 \mathrm{~mm}$ \\
\hline Thickness & $0.23 \mathrm{~mm}$ \\
\hline
\end{tabular}

\begin{tabular}{|l|l|}
\hline \multicolumn{2}{|l|}{ PRE-IRRADIATION CHARACTERISTICS } \\
\hline BU (peak axial) & $4.8,4.8$, and 6.3 at\% \\
\hline Peak linear power & (not available) \\
\hline $\begin{array}{l}\text { Peak internal clad } \\
\text { temperature }\end{array}$ & (not available) \\
\hline $\begin{array}{l}\text { Fluence } \\
\text { (spectrum) }\end{array}$ & $\begin{array}{l}\text { Fluence not available } \\
\text { (thermal spectrum) }\end{array}$ \\
\hline
\end{tabular}

\section{OTHER FUEL SAMPLE INFORMATION}

Fuel $\mathrm{O} / \mathrm{M}=2.019$. Overall pin length $=22.5 \mathrm{~cm}$. Spacer wire of $1.2 \mathrm{~mm}$ diameter. Gas release fraction $\sim 23 \%$.

Central void radius $\sim 0.25 \mathrm{~mm}$

\begin{tabular}{|l|l|}
\hline \multicolumn{2}{|c|}{ TEST CONDITIONS, MEASUREMENTS, \& ANALYSES } \\
\hline $\begin{array}{l}\text { Coolant } \\
\text { environment }\end{array}$ & $\begin{array}{l}\text { Flowing sodium at } 400{ }^{\circ} \mathrm{C} \text { and } 4.4 \mathrm{~m} / \mathrm{s}(366 \\
\left.\mathrm{cm}^{3} / \mathrm{s} \text {; flow cross sectional area }=0.82 \mathrm{~cm}^{2}\right)\end{array}$ \\
\hline $\begin{array}{l}\text { Transient } \\
\text { conditions }\end{array}$ & $\begin{array}{l}\text { Reactivity-step burst transient having } 35-\mathrm{ms} \\
\text { initial period, } 150 \text { ms full-width-at-half-maximum, } \\
\text { producing a peak fuel power of } 11.4 \mathrm{~kW} / \mathrm{g} \text { and a } \\
\text { pin-cluster-averaged fuel energy of } 2.2 \mathrm{~kJ} / \mathrm{g} .\end{array}$ \\
\hline $\begin{array}{l}\text { Fuel sample } \\
\text { configuration } \\
\text { in the test }\end{array}$ & $\begin{array}{l}\text { Three-pin bunde; fluted flow boundary to } \\
\text { simulate neighboring pins }\end{array}$ \\
\hline $\begin{array}{l}\text { Test vehicle \& } \\
\text { experiment } \\
\text { system }\end{array}$ & $\begin{array}{l}\text { Mark-IIA sodium loop; no thermal neutron filter; } \\
\text { sodium annul us outside of the flowtube }\end{array}$ \\
\hline $\begin{array}{l}\text { Measurements } \\
\text { /diagnostics } \\
\text { during test }\end{array}$ & $\begin{array}{l}\text { Temperatures at various locations; flow rates } \\
\text { and pressures at inlet and outlet; fuel motion } \\
\text { (hodoscope) }\end{array}$ \\
\hline $\begin{array}{l}\text { Posttest } \\
\text { coolant flow } \\
\text { condition }\end{array}$ & Complete flow blockage \\
\hline $\begin{array}{l}\text { Posttest } \\
\text { condition of } \\
\text { test sample(s) }\end{array}$ & Highly disrupted \\
\hline $\begin{array}{l}\text { Posttest } \\
\text { measurements } \\
\text { \& information }\end{array}$ & $\begin{array}{l}\text { Neutron radiography, macro-examinations, } \\
\text { microphotography }\end{array}$ \\
\hline $\begin{array}{l}\text { Posttest } \\
\text { analyses }\end{array}$ & $\begin{array}{l}\text { Thermal-to-work energy conversion, severe- } \\
\text { accident fuel-pin computations }\end{array}$ \\
\hline
\end{tabular}

KEY SUMMARY INFORMATION ABOUT THE TEST

Test E3 aimed to obtain data on the severity of energy conversion to work resulting from failure of high-gas-content irradiated-oxide LMFBR fuel into coolant-filled channels in a hypothetical transient-overpower (TOP) accident. The data were to help determine whether trapped fission gas would tend to fragment the fuel expelled from the fuel pin and thereby promote an energetic fuel-coolant thermal interaction, or whether the fission gas would blanket the fuel and inhibit fuel-coolant heat transfer, It was the first test to study the consequences of irradiated oxide fuel under TOP conditions. Cladding fail ure, indicated by sudden coolant channel voiding prior to codant boiling, occurred $15 \mathrm{~ms}$ before peak power, when the highest local energy input in the hottest pin was $\sim 860 \mathrm{~J} / \mathrm{g}$. The void growth rate was $~ 9.5 \mathrm{~cm}^{3} / \mathrm{ms}$, leading to complete voiding of the fueled region within about $12 \mathrm{~ms}$ from the time of fail ure. Gross fuel motion, generally a steady upward fuel dispersal, began within $\sim 30 \mathrm{~ms}$ after the cladding failure and was essentially complete in about $100 \mathrm{~ms}$. Peak recorded pressures were at most $6 \mathrm{MPa}$. The fuel was likely at incipient melting, or had just begun to melt, when the cladding failed. The ratio of coolant work done to the total fission energy generated by the time the work was done was about $0.3 \%$.

Posttest examination showed that all of the cladding had been destroyed except for at the extreme upper end of the fuel pin. About two-thirds of the total fuel and cladding inventory moved upward, out of the original fuel region, where it formed a flow blockage. Much of the fuel in the blockage was highly fragmented. Some cladding moved downward, but not in sufficient quantity to cause a flow blockage at the inlet. There was little or no interaction between the fuel and the surrounding steel structures in the original fuel-column region. There was some mixing of fuel and steel, but not on a large scale. 


\section{Summary of TREAT Test E4}

Performed 17 November 1970

Transient No. 1346

\section{PRE-TEST SAMPLE CHARACTERIZATION}

\begin{tabular}{|l|l|}
\hline \multicolumn{2}{|c|}{ FUEL } \\
\hline Form & Fast reactor mixed oxide \\
\hline Composition & $72 \% \mathrm{UO}_{2}-28 \% \mathrm{PuO}_{2 ;} 65 \%$ enr. U \\
\hline Geometry & Clad pellet stack \\
\hline Fissile height & $343 \mathrm{~mm}$ \\
\hline $\begin{array}{l}\text { Smear } \\
\text { density }\end{array}$ & $9.76 \mathrm{~g} / \mathrm{cm}^{3}(88.04 \%$ of theoretical $)$ \\
\hline
\end{tabular}

\begin{tabular}{|l|l|}
\hline \multicolumn{2}{|c|}{ CLADDING } \\
\hline Material & Type 316 stainless steel \\
\hline OD & $5.84 \mathrm{~mm}$ \\
\hline Thickness & $0.38 \mathrm{~mm}$ \\
\hline
\end{tabular}

\begin{tabular}{|l|l|}
\hline \multicolumn{2}{|l|}{ PRE-IRRADIATION CHARACTERISTICS } \\
\hline BU (peak axial) & (Not pre-irradiated) \\
\hline Peak linear power & (Not applicable) \\
\hline $\begin{array}{l}\text { Peak internal clad } \\
\text { temperature }\end{array}$ & (Not applicable) \\
\hline $\begin{array}{l}\text { Fluence } \\
\text { (spectrum) }\end{array}$ & (Not applicable) \\
\hline
\end{tabular}

OTHER FUEL SAMPLE INFORMATION

1-mm-diameter wire wrap

\begin{tabular}{|l|l|}
\hline \multicolumn{2}{|c|}{ TEST CONDITIONS, MEASUREMENTS, \& ANALYSES } \\
\hline $\begin{array}{l}\text { Coolant } \\
\text { environment }\end{array}$ & $\begin{array}{l}\text { Flowing sodium at } 5.4 \mathrm{~m} / \mathrm{s} \text { (142 } \mathrm{cm}^{3} / \mathrm{s} \text { ) and } 425 \\
\text { 'C }\end{array}$ \\
\hline $\begin{array}{l}\text { Transient } \\
\text { conditions }\end{array}$ & $\begin{array}{l}\text { Power transient produced by a } 2.25 \% \text { KK/k } \\
\text { reactivity step, with } 66 \text { ms initial period, } \\
\text { generating } 740 \mathrm{MW} \text {-s with full-width-at-half- } \\
\text { maximum of 230 ms. }\end{array}$ \\
\hline $\begin{array}{l}\text { Fuel sample } \\
\text { configuration } \\
\text { in the test }\end{array}$ & Single pin \\
\hline $\begin{array}{l}\text { Test vehicle \& } \\
\text { experiment } \\
\text { system }\end{array}$ & $\begin{array}{l}\text { Mark-IIA integral sodium loop, with BsSi filtering. } \\
\text { flowtube was a 0.55-mm-wide evacuated } \\
\text { annulus, then a 0.8 mm-thick stainless steel } \\
\text { tube, then a 10-mm-thick sodium annulus. }\end{array}$ \\
\hline $\begin{array}{l}\text { Measurements } \\
\text { /diagnostics } \\
\text { during test }\end{array}$ & $\begin{array}{l}\text { Temperatures, inlet and outlet pressures, inlet } \\
\text { and outlet flow rates. (Hodoscope data were not } \\
\text { available.) }\end{array}$ \\
\hline $\begin{array}{l}\text { Posttest } \\
\text { coolant flow } \\
\text { condition }\end{array}$ & Complete flow blockage at both inlet and outlet \\
\hline $\begin{array}{l}\text { Posttest } \\
\text { condition of } \\
\text { test sample(s) }\end{array}$ & Highly disrupted \\
\hline $\begin{array}{l}\text { Posttest } \\
\text { measurements } \\
\text { \& information }\end{array}$ & $\begin{array}{l}\text { Neutron radiography, axial gamma scan, macro- } \\
\text { photography, microphotography, particle size } \\
\text { analysis }\end{array}$ \\
\hline $\begin{array}{l}\text { Posttest } \\
\text { analyses }\end{array}$ & Thermal-hydraulic, fuel-pin transient behavior \\
\hline
\end{tabular}

\section{KEY SUMMARY INFORMATION ABOUT THE TEST}

Test E4 subjected a fresh LMFBR fuel pin to a fast transient overpower excursion sufficient to cause pin failure. It was nearly identical to Test $\mathrm{H} 2$ which used a slower overpower transient. Upward in-pin fuel motion may have begun as early as $100 \mathrm{~ms}$ before cladding fail ure. Mild sodium boiling existed for 25 to $50 \mathrm{~ms}$ before failure. The cladding failed $85 \mathrm{~ms}$ after peak power (when $~ 70 \%$ of the transient energy had been generated and $1.6 \mathrm{~kJ} / \mathrm{g}$ had been generated in the test fuel). A little fuel was promptly released upon fail ure. Within $10 \mathrm{~ms}$ after failure, the flow channel along the entire pin completely voided itself of sodium, and the outlet started to become blocked, apparenty by freezing of upward-driven molten fuel. Three more sodium expulsion events occurred at the outtet, and the blockage was complete by $\sim 50 \mathrm{~ms}$ after cladding fail ure. The flowtube likely failed during this time, which would have opened an entrance to the surrounding initiallyevacuated annulus and may have caused the observed sudden flow reversal of the initially-expelled sodium slug. A minor secondary event occurred $65 \mathrm{~ms}$ after cladding fail ure, involving redistribution of much fuel and steel. No energetic fuel-coolant interactions occurred. Time-dependent fuel-motion data recorded by the hodoscope were found posttest to have been corrupted by an extraneous signal and rendered unusable. From the posttest examination, it appeared that the major point of failure was within $5 \mathrm{~cm}$ of the top of the fuel col umn. A large fraction of the fuel moved above the original fuel region. The entire cladding had melted along the original fuel region, and some of that steel formed a blockage near the inlet. There was no evidence of massive fuel relocation toward the inlet. The flowtube wall had been penetrated in many locations along the upper half of the original fuel region, and extensively melted in the region extending more than $\sim 10 \mathrm{~cm}$ higher. Fuel had been pushed upward within the remaining intact cladding of the fuel pin plenum. 


\section{Summary of TREAT Test E6}

Performed 18 January 1973

Transient No. 1487

\section{PRE-TEST SAMPLE CHARACTERIZATION}

\begin{tabular}{|l|l|}
\hline \multicolumn{2}{|c|}{ FUEL } \\
\hline Form & He-bonded MOX \\
\hline Composition & $\begin{array}{l}75 \mathrm{wt} \% \mathrm{UO}_{2} \text { (77\% enriched center } \\
\text { pin; } 65 \% \text { enriched edge pins) } \\
25 \text { wt\% } \mathrm{PuO}_{2}\end{array}$ \\
\hline Geometry & Solid pellet \\
\hline Fissile height & $343 \mathrm{~mm}$ \\
\hline $\begin{array}{l}\text { Smear } \\
\text { density }\end{array}$ & $\begin{array}{l}9.23 \mathrm{~g} / \mathrm{cm}^{3} \text { center pin } \\
9.65 \mathrm{~g} / \mathrm{cm}^{3} \text { edge pins }\end{array}$ \\
\hline
\end{tabular}

\begin{tabular}{|l|l|}
\hline \multicolumn{2}{|c|}{ CLADDING } \\
\hline Material & 316 stainless steel, 20\% CW \\
\hline OD & $5.84 \mathrm{~mm}$ \\
\hline Thickness & $0.39 \mathrm{~mm}$ \\
\hline
\end{tabular}

\begin{tabular}{|l|l|}
\hline \multicolumn{2}{|l|}{ PRE-IRRADIATION CHARACTERISTICS } \\
\hline BU (peak axial) & $\begin{array}{l}4.6 \text { at\% HM (center pin) } \\
\text { No burnup in edge pins. }\end{array}$ \\
\hline Peak linear power & $39 \mathrm{~kW} / \mathrm{m}$ (center pin) \\
\hline $\begin{array}{l}\text { Peak internal clad } \\
\text { temperature }\end{array}$ & (not available) \\
\hline $\begin{array}{l}\text { Fluence } \\
\text { (spectrum) }\end{array}$ & $\begin{array}{l}4 \times 10^{22} \mathrm{n} / \mathrm{cm}^{2} \text { (center pin); } \\
\text { fast spectrum (EBR-II) }\end{array}$ \\
\hline
\end{tabular}

\section{OTHER FUEL SAMPLE INFORMATION}

FFTF/FTR-like wire-wrapped pins. Lower $\mathrm{UO}_{2}$ insulator: $13 \mathrm{~mm}$ long. Upper $\mathrm{UO}_{2}$ insulator: $13 \mathrm{~mm}$ in edge pins, $170 \mathrm{~mm}$ in center pin. Upper Inconel reflector above insulator was $152 \mathrm{~mm}$ long in edge pins, $127 \mathrm{~mm}$ in center pin. Total fuel mass in test $=466 \mathrm{~g}$.

\begin{tabular}{|l|l|}
\hline \multicolumn{2}{|c|}{ TEST CONDITIONS, MEASUREMENTS, \& ANALYSES } \\
\hline $\begin{array}{l}\text { Coolant } \\
\text { environment }\end{array}$ & Flowing sodium at $400{ }^{\circ} \mathrm{C}$ and $615 \mathrm{~cm} 3 / \mathrm{s}$ \\
\hline $\begin{array}{l}\text { Transient } \\
\text { conditions }\end{array}$ & $\begin{array}{l}\text { Preheat at } 167 \mathrm{MW} \text { TREAT power }(54 \mathrm{~kW} / \mathrm{m} \\
\text { center pin, } 65 \mathrm{~kW} / \mathrm{m} \text { edge pins) for } 3 \mathrm{~s}, \text { then a } \\
\text { power ramp with } 183 \text { ms period reaching } 2250 \\
\text { MW peak 0.52 s later, then scram. Power burst } \\
\text { full-width-at-half-maximum was } 0.28 \mathrm{~s} .\end{array}$ \\
\hline $\begin{array}{l}\text { Fuel sample } \\
\text { configuration } \\
\text { in the test }\end{array}$ & $\begin{array}{l}\text { Seven-pin bundle, with one irradiated pin } \\
\text { surrounded by six fresh edge pins }\end{array}$ \\
\hline $\begin{array}{l}\text { Test vehicle \& } \\
\text { experiment } \\
\text { system }\end{array}$ & $\begin{array}{l}\text { Mark-IIA integral sodium loop with B6Si filter. } \\
\text { Stainless steel flowtube } 0.89 \text { mm thick } \\
\text { surrounded by a } 2.4 \text { mm gas-filled annul us } \\
\text { bounded by a 0.89-mm thick stainless steel } \\
\text { outer wall. }\end{array}$ \\
\hline $\begin{array}{l}\text { Measurements } \\
\text { /diagnostics } \\
\text { during test }\end{array}$ & $\begin{array}{l}\text { Temperatures, coolant flow rate and pressure, } \\
\text { fuel motion (hodoscope) }\end{array}$ \\
\hline $\begin{array}{l}\text { Posttest } \\
\text { coolant flow } \\
\text { condition }\end{array}$ & Complete flow blockage. \\
\hline $\begin{array}{l}\text { Posttest } \\
\text { condition of } \\
\text { test sample(s) }\end{array}$ & $\begin{array}{l}\text { Highly disrupted, except near the bottom, with } \\
\text { little axial dispersal of material. }\end{array}$ \\
\hline $\begin{array}{l}\text { Posttest } \\
\text { measurements } \\
\text { \& information }\end{array}$ & $\begin{array}{l}\text { Neutron radiography, axial gamma scanning, } \\
\text { macro-examinations, microphotography }\end{array}$ \\
\hline $\begin{array}{l}\text { Posttest } \\
\text { analyses }\end{array}$ & Thermal-hydraulics, severe-accident analysis \\
\hline
\end{tabular}

\section{KEY SUMMARY INFORMATION ABOUT THE TEST}

Test E6 subjected a bundle of six fresh pins surrounding one high-power-irradiated pin to conditions simulating an LMFBR hypothetical $\$ 3 / \mathrm{s}$ transient overpower accident. (The test was the fast-transient counterpart to the $50 \& / \mathrm{s}$ TOP test H4.) As the fuel heated during the power burst, the fuel pins bowed, and some fuel $(5$ to $10 \mathrm{~g})$ may have moved slightly upward within the cladding of the hottest edge pins(s), from fuel axial center. Fuel-pin fail ure occurred $550 \mathrm{~ms}$ after the start of the overpower ramp ( 15 ms after peak power), causing sudden reversal of inlet flow and sharp acceleration of outlet flow. Two more similar flow events followed: one at $\sim 45 \mathrm{~ms}$ and one at $\sim 90$ ms after peak power (at TREAT power $\sim 1900 \mathrm{MW}$ and $\sim 1100 \mathrm{MW}$, respectively), suggesting additional pin failures. Fuel dispersal, and rupture of the flowtube at a fued relative height $x / L=0.65$ to 0.8 , were associated with the last of these events. Posttest examinations showed cladding melting as low as $x / L=0.15$ in the edge pins and as low as $x / L=0.4$ in the central pin. There was little fuel/steel debris in the flow channels in the lower third of the original fuel region. Considerable fuel/steel material had moved radially through the flowtube breach outward to the surrounding outer wall over the region $x / L=0.7$ to 1.0. Most of the upper half of the (preirradiated) fuel from the center pin had redistributed fairly uniformly along the length of the original fuel region, with extra accumulation at $x / L=1.3$ to 1.5 , and had a sponge-like appearance. Most of the fuel/steel material that had dispersed axially (less than $2 \%$ of the total test fuel mass) was distributed throughout a 250-mm-long region above the fuel column. Most of the axially-dispersed fuel was the pre-irradiated fuel. The debris accumulation in the region $x / L=0.8$ to 1.05 caused the flow blockage. 


\title{
Summary of TREAT Test E7
}

\author{
Performed 9 March 1973
}

Transient No. 1499

\section{PRE-TEST SAMPLE CHARACTERIZATION}

\begin{tabular}{|l|l|}
\hline \multicolumn{2}{|c|}{ FUEL } \\
\hline Form & He-bonded MOX \\
\hline Composition & $\begin{array}{l}75 \mathrm{wt} \% \mathrm{UO}_{2} \text { (77\% enriched) } \\
25 \mathrm{wt} \% \mathrm{PuO}_{2}\end{array}$ \\
\hline Geometry & Solid pellet \\
\hline Fissile height & $343 \mathrm{~mm}$ \\
\hline $\begin{array}{l}\text { Smear } \\
\text { density }\end{array}$ & $9.2 \mathrm{~g} / \mathrm{cm}^{3}$ \\
\hline
\end{tabular}

\begin{tabular}{|l|l|}
\hline \multicolumn{2}{|c|}{ CLADDING } \\
\hline Material & 316 stainless steel, 20\% CW \\
\hline OD & $5.84 \mathrm{~mm}$ \\
\hline Thickness & $0.38 \mathrm{~mm}$ \\
\hline
\end{tabular}

\section{PRE-IRRADIATION CHARACTERISTICS}

\begin{tabular}{|l|l|}
\hline BU (peak axial) & 4.2 at $\% \mathrm{HM}$ \\
\hline Peak linear power & $32-38 \mathrm{~kW} / \mathrm{m}$ \\
\hline $\begin{array}{l}\text { Peak internal clad } \\
\text { temperature }\end{array}$ & $\sim 500^{\circ} \mathrm{C}$ \\
\hline $\begin{array}{l}\text { Fluence } \\
\text { (spectrum) }\end{array}$ & $\begin{array}{l}\text { (Fluence not available) } \\
\text { Fast (EBR-II) spectrum }\end{array}$ \\
\hline
\end{tabular}

\section{OTHER FUEL SAMPLE INFORMATION}

FFTR/FTR-type wire-wrapped HEDL N-F fuel pins. 170 $\mathrm{mm} \mathrm{UO}_{2}$ insulator above the fuel; $13 \mathrm{~mm} \mathrm{UO}_{2}$ insulator below the fuel. Inconel reflector $127 \mathrm{~mm}$ long above the upper insulator pellets. High-power-structure fuel after irradiation.

\begin{tabular}{|c|c|}
\hline \multicolumn{2}{|c|}{ TEST CONDITIONS, MEASUREMENTS, \& ANALYSES } \\
\hline $\begin{array}{l}\text { Coolant } \\
\text { environment }\end{array}$ & $\begin{array}{l}\text { Sodium at } ~ 380^{\circ} \mathrm{C} \text { and initial flowrate of } 3.9 \mathrm{~m} / \mathrm{s} \\
\left(570 \mathrm{~cm}^{3} / \mathrm{s}\right)\end{array}$ \\
\hline $\begin{array}{l}\text { Transient } \\
\text { conditions }\end{array}$ & $\begin{array}{l}\text { } 2.4 \mathrm{~s} \text { at } ~ 140 \mathrm{MW} \text { TREAT power ( } 41 \mathrm{~kW} / \mathrm{m} \text { in } \\
\text { central pin, } 53 \mathrm{~kW} / \mathrm{m} \text { in } 5 \text { hottest edge pins), } \\
\text { then a power burst rising on } 180 \text { ms period, } \\
\text { reaching } 2560 \mathrm{MW} \text { at } 0.54 \mathrm{~s} \text { after start of burst. } \\
\text { Power dropped to }<200 \mathrm{MW} \text { within next } 0.4 \mathrm{~s} \text {. } \\
\text { Burst full-width-at-half-maximum }=310 \mathrm{~ms} \text {. }\end{array}$ \\
\hline $\begin{array}{l}\text { Fuel sample } \\
\text { configuration } \\
\text { in the test }\end{array}$ & 7-pin bunde of nominally-identical pins \\
\hline $\begin{array}{l}\text { Test vehicle \& } \\
\text { experiment } \\
\text { system }\end{array}$ & $\begin{array}{l}\text { Mark-II integral sodium loop with } \mathrm{B}_{6} \mathrm{Si} \text { filtering. } \\
\text { Fluted, } 0.9-\mathrm{mm} \text { thick, stainless steel flowtube } \\
\text { surrounded the pin bunde. Outside the flowtube } \\
\text { was a } 0.76 \text { - } \mathrm{mm} \text {-thick stainless tube; a } 3.2 \mathrm{~mm} \\
\text { annul us existed between the tubes. }\end{array}$ \\
\hline $\begin{array}{l}\text { Measurements } \\
\text { /diagnostics } \\
\text { during test }\end{array}$ & $\begin{array}{l}\text { Coolant and flowtube temperatures, coolant flow } \\
\text { rate and pressure, fuel motion (hodoscope) }\end{array}$ \\
\hline $\begin{array}{l}\text { Posttest } \\
\text { coolant flow } \\
\text { condition }\end{array}$ & Complete flow blockage \\
\hline $\begin{array}{l}\text { Posttest } \\
\text { condition of } \\
\text { test sample(s) }\end{array}$ & $\begin{array}{l}\text { Highly disrupted. All of cladding and nearly all of } \\
\text { fuel had melted. Considerable fuel and steel } \\
\text { above original top of fuel region. }\end{array}$ \\
\hline $\begin{array}{l}\text { Posttest } \\
\text { measurements } \\
\text { \& information }\end{array}$ & $\begin{array}{l}\text { Neutron radiography, macroscopic examination, } \\
\text { optical microscopy }\end{array}$ \\
\hline $\begin{array}{l}\text { Posttest } \\
\text { analyses }\end{array}$ & $\begin{array}{l}\text { Thermal-hydraulic; hodoscope fuel-motion data } \\
\text { analysis }\end{array}$ \\
\hline
\end{tabular}

\section{KEY SUMMARY INFORMATION ABOUT THE TEST}

Test E7 subjected high-power-microstructure irradiated MOX fuel to a hypothetical $\$ 3 / s$ transient overpower LMFBR accident. Flow disturbances began about $400 \mathrm{~ms}$ into the power burst (at TREAT power of $1500 \mathrm{MW}$ ). During the next $130 \mathrm{~ms}$ (as the TREAT power reached its peak), three successive jumps in the outlet flow rate occurred, and the inlet flow gradually slowed and then reversed direction, corresponding to codant voiding in the fuel region. Thereafter, the outlet flow suddenly decreased substantially and then gradually decreased further, reaching zero at $\sim 300 \mathrm{~ms}$ after peak TREAT power. The peak pressure recorded during the test was $0.5 \mathrm{MPa}$, at the inlet. Post-failure fuel motion was mostly lateral, occurring at various times, with small amounts of fuel moving to the top and bottom of the original fuel region. Posttest radiography and macro-examination of the remains revealed that a few partially-intact pellets remained at the original bottom of the fuel region and that a little fuel and steel had flowed below that region. The flowtube had melted al most totally within the original fuel region and above (fuel relative height $x / L=0$ to 1.25). The cladding and spacer wires had melted completely within $x / L=0$ to 1.0 , and the fuel columns had melted from $x / L=0.05$ to 1.0. Most of the steel movement had preceded the fuel movement, with steel extending to the wall radially beyond the flowtube. Large globules of steel were found within the fuel in some regions. Accumulations of fuel and steel were of low density between $x / L=0$ and 0.35 , were not present between $x / L=0.35$ and 0.50 , were of medium density between $x / L=0.5$ and 0.8 , were of high density between $x / L=0.8$ and 1.0 , and were of medium density above that point. 


\title{
Summary of TREAT Test E8
}

\author{
Performed 9 July 1974
}

Transient No. 1588

\section{PRE-TEST SAMPLE CHARACTERIZATION}

\begin{tabular}{|l|l|}
\hline \multicolumn{2}{|c|}{ FUEL } \\
\hline Form & He-bonded MOX \\
\hline Composition & $\begin{array}{l}75 \text { wt\% } \mathrm{UO}_{2} \text { (65\% enriched) } \\
25 \text { wt } \mathrm{PuO}_{2}\end{array}$ \\
\hline Geometry & Solid pellet \\
\hline Fissile height & $343 \mathrm{~mm}$ \\
\hline $\begin{array}{l}\text { Smear } \\
\text { density }\end{array}$ & $85.5 \%$ of theoretical \\
\hline
\end{tabular}

\begin{tabular}{|l|l|}
\hline \multicolumn{2}{|c|}{ CLADDING } \\
\hline Material & 316 stainless steel, 20\% CW \\
\hline OD & $5.84 \mathrm{~mm}$ \\
\hline Thickness & $0.38 \mathrm{~mm}$ \\
\hline
\end{tabular}

\begin{tabular}{|l|l|}
\hline \multicolumn{2}{|l|}{ PRE-IRRADIATION CHARACTERISTICS } \\
\hline BU (peak axial) & $\sim 5$ at\% HM \\
\hline Peak linear power & $30 \mathrm{~kW} / \mathrm{m}$ \\
\hline $\begin{array}{l}\text { Peak internal clad } \\
\text { temperature }\end{array}$ & (not available) \\
\hline $\begin{array}{l}\text { Fluence } \\
\text { (spectrum) }\end{array}$ & $\begin{array}{l}\text { (Fluence not available) } \\
\text { Fast (EBR-II) spectrum }\end{array}$ \\
\hline
\end{tabular}

\section{OT HER FUEL SAMPLE INFORMATION}

FFTF/FTR-type wire-wrapped PNL-10 fuel pins; low power microstructure but with partial small central void. $356 \mathrm{~mm} \mathrm{UO}_{2}$ insulator above the fuel; $169 \mathrm{~mm} \mathrm{UO}_{2}$ insulator below the fuel. Inconel or stainless steel reflector $127 \mathrm{~mm}$ long above the upper insulator pellets.

\begin{tabular}{|l|l|}
\hline \multicolumn{2}{|c|}{ TEST CONDITIONS, MEASUREMENTS, \& ANALYSES } \\
\hline $\begin{array}{l}\text { Coolant } \\
\text { environment }\end{array}$ & $\begin{array}{l}\text { Sodium at } 400{ }^{\circ} \mathrm{C} \text { and an initial flowrate of } 575 \\
\mathrm{~cm}^{3} / \mathrm{s}\end{array}$ \\
\hline $\begin{array}{l}\text { Transient } \\
\text { conditions }\end{array}$ & $\begin{array}{l}\text { 2 } \mathrm{s} \text { at } 115 \mathrm{MW} \text { TREAT power (46 } \mathrm{kW} / \mathrm{m} \text { in test } \\
\text { fuel, then a power burst reaching about } 2180 \\
\text { MW TREAT power within } 500 \mathrm{~ms} \text { (1.4 MJ/kg } \\
\text { energy in the test fuel). Peak power (2535 MW) } \\
\text { occurred about } 80 \text { ms later. Then scram. }\end{array}$ \\
\hline $\begin{array}{l}\text { Fuel sample } \\
\text { configuration } \\
\text { in the test }\end{array}$ & 7-pin bundle of preirradiated pins \\
\hline $\begin{array}{l}\text { Test vehicle \& } \\
\text { experiment } \\
\text { system }\end{array}$ & $\begin{array}{l}\text { Mark-IIC integral sodium loop with Dy filtering. } \\
\text { Fluted, 0.9-mm thick, stainless steel flowtube } \\
\text { surrounded the pin bundle. }\end{array}$ \\
\hline $\begin{array}{l}\text { Measurements } \\
\text { /diagnostics } \\
\text { during test }\end{array}$ & $\begin{array}{l}\text { Coolant pressure, flow rate, temperatures, fuel } \\
\text { motion }\end{array}$ \\
\hline $\begin{array}{l}\text { Posttest } \\
\text { coolant flow } \\
\text { condition }\end{array}$ & Complete flow blockage \\
\hline $\begin{array}{l}\text { Posttest } \\
\text { condition of } \\
\text { test sample(s) }\end{array}$ & $\begin{array}{l}\text { Grossly disrupted; pellet stack structure only in } \\
\text { the bottom fourth of the fuel region. }\end{array}$ \\
\hline $\begin{array}{l}\text { Posttest } \\
\text { measurements } \\
\text { \& information }\end{array}$ & $\begin{array}{l}\text { Neutron radiography, gamma scanning, } \\
\text { photography, optical microscopy }\end{array}$ \\
\hline $\begin{array}{l}\text { Posttest } \\
\text { analyses }\end{array}$ & Thermal-hydraulics; hodoscope data analysis \\
\hline
\end{tabular}

\section{KEY SUMMARY INFORMATION ABOUT THE TEST}

Test E8 subjected low-power-microstructure irradiated MOX fuel to a hypothetical \$3/s transient overpower LMFBR accident. The initial cladding failure occurred at $2180 \mathrm{MW}$ TREAT power (test fuel power about 29 times the $30 \mathrm{~kW} / \mathrm{m}$ nominal preirradiation power), when about $40 \%$ of the TREAT overpower burst energy had been generated. Failure was accompanied by $2 \%$ of the fuel mass being promptly ejected above the original top of the fuel column, rapid sodium voiding and inlet flow reversal, and low (1.5 MPa) pressure pulses at the inlet. Upward fuel dispersal occurred only during the first $80 \mathrm{~ms}$ after fuel motion began, after which a fuel-debris blockage apparently occurred, involving about $60 \mathrm{~g}$ of fuel. Subsequent fuel motions (all during the next $120 \mathrm{~ms}$ ) occurred mostly within the original fuel region. The posttest distribution of fuel and steel was basically an accumulation of mostly steel plus some fuel located as far as $33 \mathrm{~cm}$ above the original fued region, a dense accumulation of fuel and steel in the top half of the original fuel region $(x / L=0.5$ to 1.0 , including complete melting of the flowtube), the region $x / L=0.25$ to 0.5 essential voided of both fuel and steel, and some fuel and steel in the region $x / L=0$ to 0.25. All of the fuel in the upper two-thirds of the fuel columns apparently melted. Most of the steel that had melted moved above the top of the initial fuel region. 


\section{Appendix N: One-page Summaries of H-series Tests}

One-page summaries of each of the H-series tests are included here. These summaries can also be found in TREXR [6]. 


\section{Summary of TREAT Test H1}

Performed 2 February 1970

Transient No. 1298

\section{PRE-TEST SAMPLE CHARACTERIZATION}

\begin{tabular}{|l|l|}
\hline \multicolumn{2}{|c|}{ FUEL } \\
\hline Form & Fast reactor U-Pu oxide \\
\hline Composition & U-15\%Pu, U enriched to $60 \% 235 \mathrm{U}$ \\
\hline Geometry & Annular pellet, $0.76 \mathrm{~mm} \mathrm{ID}$ \\
\hline Fissile height & $37.6 \mathrm{~cm}$ \\
\hline $\begin{array}{l}\text { Smear } \\
\text { density }\end{array}$ & $9.15 \mathrm{~g} / \mathrm{cm}^{3}$ \\
\hline
\end{tabular}

\begin{tabular}{|l|l|}
\hline \multicolumn{2}{|c|}{ CLADDING } \\
\hline Material & 316 stainless steel \\
\hline OD & $5.84 \mathrm{~mm}$ \\
\hline Thickness & $0.38 \mathrm{~mm}$ \\
\hline
\end{tabular}

\begin{tabular}{|l|l|}
\hline \multicolumn{2}{|l|}{ PRE-IRRADIATION CHARACTERISTICS } \\
\hline BU (peak axial) & None \\
\hline Peak linear power & (not applicable) \\
\hline $\begin{array}{l}\text { Peak internal clad } \\
\text { temperature }\end{array}$ & (not applicable) \\
\hline $\begin{array}{l}\text { Fluence } \\
\text { (spectrum) }\end{array}$ & (none) \\
\hline
\end{tabular}

\section{OTHER FUEL SAMPLE INFORMATION}

PFR-design fuel pin, but with a wire wrap of $0.8 \mathrm{~mm}$ diameter. Fuel mass $=69.7 \mathrm{~g}$. An extensometer was

located within the pin plenum.

\begin{tabular}{|c|c|}
\hline \multicolumn{2}{|c|}{ TEST CONDITIONS, MEASUREMENTS, \& ANALYSES } \\
\hline $\begin{array}{l}\text { Coolant } \\
\text { environment }\end{array}$ & $\begin{array}{l}\text { Flowing sodium at } 0.4 \mathrm{~m} / \mathrm{s} \text {, with initial loop } \\
\text { plenum pressure of } 3 \mathrm{bar} \text {. }\end{array}$ \\
\hline $\begin{array}{l}\text { Transient } \\
\text { conditions }\end{array}$ & $\begin{array}{l}\text { Natural burst transient of } 0.36 \mathrm{~s} \text { full-width-at-half- } \\
\text { maximum, generating } 960 \mathrm{~J} / \mathrm{g} \text { in the test fuel. }\end{array}$ \\
\hline $\begin{array}{l}\text { Fuel sample } \\
\text { configuration } \\
\text { in the test }\end{array}$ & $\begin{array}{l}\text { Single pin oriented with plenum below the fuel, } \\
\text { toward the coolant inlet. }\end{array}$ \\
\hline $\begin{array}{l}\text { Test vehicle \& } \\
\text { experiment } \\
\text { system }\end{array}$ & $\begin{array}{l}\text { Mark-II sodium loop with } \mathrm{B}_{6} \text { Si filtering. Single- } \\
\text { pin test train with insulated flow-tube wall } 8.28 \\
\text { mm ID, } 0.79 \text { mm thickness }\end{array}$ \\
\hline $\begin{array}{l}\text { Measurements } \\
\text { /diagnostics } \\
\text { during test }\end{array}$ & Temperature, flow rate \\
\hline $\begin{array}{l}\text { Posttest } \\
\text { coolant flow } \\
\text { condition }\end{array}$ & Same as at the start of the test. \\
\hline $\begin{array}{l}\text { Posttest } \\
\text { condition of } \\
\text { test sample(s) }\end{array}$ & $\begin{array}{l}\text { The fuel pin remained intact, with little effect due } \\
\text { to the test. }\end{array}$ \\
\hline $\begin{array}{l}\text { Posttest } \\
\text { measurements } \\
\text { \& information }\end{array}$ & $\begin{array}{l}\text { Visual inspection, radiation monitoring, neutron } \\
\text { radiography, eddy current inspection of cladding, } \\
\text { X-radiography }\end{array}$ \\
\hline $\begin{array}{l}\text { Posttest } \\
\text { analyses }\end{array}$ & Thermal-hydraulics \\
\hline
\end{tabular}

\section{KEY SUMMARY INFORMATION ABOUT THE TEST}

Test $\mathrm{H} 1$ was intended to be a preliminary, failure-threshold test to begin the $\mathrm{H}$-series. Posttest visual inspection and radiation monitoring found no damage to the fuel pin caused by the experiment. Eddy current examination indicated possible abnormal regions in the cladding amounting to $10-20 \%$ of the wall thickness located in the fuel region 4 to $9 \mathrm{~cm}$ from the plenum end of the fuel. The insulator pellet at the end of the fuel column farthest from the plenum was badly fractured due to the transient. It was determined posttest that the extensometer was probably in contact with the end of the fuel column and bound within the cladding prior to the test (and thus possibly mechanically restraining axial fuel expansion). The fuel pellets became extensively cracked, and irregularities formed in the fuel central void, as a result of the transient. 


\title{
Summary of TREAT Test H2
}

\author{
Performed 11 June 1970
}

Transient No. 1317

\section{PRE-TEST SAMPLE CHARACTERIZATION}

\begin{tabular}{|c|c|}
\hline \multicolumn{2}{|r|}{ FUEL } \\
\hline Form & Fast reactor U-Pu oxide \\
\hline Composition & $75 \% \mathrm{UO}_{2}-25 \% \mathrm{PuO}_{2} ; 65 \%$ enr. U \\
\hline Geometry & Clad pellet stack \\
\hline Fissile height & $34.4 \mathrm{~cm}$ \\
\hline $\begin{array}{l}\text { Smear } \\
\text { density }\end{array}$ & $9.86 \mathrm{~g} / \mathrm{cm}^{3}$ ( $89 \%$ of theoretical) \\
\hline
\end{tabular}

\begin{tabular}{|l|l|}
\hline \multicolumn{2}{|c|}{ CLADDING } \\
\hline Material & Type 316 stainless steel \\
\hline OD & $5.84 \mathrm{~mm}$ \\
\hline Thickness & $0.38 \mathrm{~mm}$ \\
\hline
\end{tabular}

\begin{tabular}{|l|l|}
\hline \multicolumn{2}{|l|}{ PRE-IRRADIATION CHARACTERISTICS } \\
\hline BU (peak axial) & (Not pre-irradiated) \\
\hline Peak linear power & (Not applicable) \\
\hline $\begin{array}{l}\text { Peak internal clad } \\
\text { temperature }\end{array}$ & (Not applicable) \\
\hline $\begin{array}{l}\text { Fluence } \\
\text { (spectrum) }\end{array}$ & (Not applicable) \\
\hline
\end{tabular}

OTHER FUEL SAMPLE INFORMATION

1-mm-diameter wire wrap

\begin{tabular}{|l|l|}
\hline \multicolumn{2}{|c|}{ TEST CONDITIONS, MEASUREMENTS, \& ANALYSES } \\
\hline $\begin{array}{l}\text { Coolant } \\
\text { environment }\end{array}$ & $\begin{array}{l}\text { Flowing sodium at } 6.3 \mathrm{~m} / \mathrm{s} \text { (165 } \mathrm{cm}^{3} / \mathrm{s} \text { ) and } 400 \\
\text { 'C }\end{array}$ \\
\hline $\begin{array}{l}\text { Transient } \\
\text { conditions }\end{array}$ & $\begin{array}{l}\text { Power transient produced by a } 1.99 \% \Delta \mathrm{k} / \mathrm{k} \\
\text { reactivity step, with } 80 \mathrm{~ms} \text { initial period, } \\
\text { generating } 490 \mathrm{MW} \text {-s with full-width-at-half- } \\
\text { maximum of } \sim 1.3 \mathrm{~s} \text {. }\end{array}$ \\
\hline $\begin{array}{l}\text { Fuel sample } \\
\text { configuration } \\
\text { in the test }\end{array}$ & Single pin \\
\hline $\begin{array}{l}\text { Test vehicle \& } \\
\text { experiment } \\
\text { system }\end{array}$ & $\begin{array}{l}\text { Mark-IIA integral sodium loop, with B6Si filtering. } \\
\text { flowtube was a 0.55-mm wide evacuated } \\
\text { annulus, then a 0.8-mm thick stainless steel } \\
\text { tube, then a 10-mm-thick sodium annulus. }\end{array}$ \\
\hline $\begin{array}{l}\text { Measurements } \\
\text { /diagnostics } \\
\text { during test }\end{array}$ & $\begin{array}{l}\text { Temperatures, inlet pressure, inlet and outlet } \\
\text { flow rates; fuel motion monitoring by the } \\
\text { hodoscope }\end{array}$ \\
\hline $\begin{array}{l}\text { Posttest } \\
\text { coolant flow } \\
\text { condition }\end{array}$ & Complete blockage. \\
\hline $\begin{array}{l}\text { Posttest } \\
\text { condition of } \\
\text { test sample(s) }\end{array}$ & Extensively damaged \\
\hline $\begin{array}{l}\text { Posttest } \\
\text { measurements } \\
\text { \& information }\end{array}$ & $\begin{array}{l}\text { Neutron radiography, macro-photography, } \\
\text { microphotography, particle-size analysis }\end{array}$ \\
\hline $\begin{array}{l}\text { Posttest } \\
\text { analyses }\end{array}$ & Thermal hydraulics \\
\hline
\end{tabular}

\section{KEY SUMMARY INFORMATION ABOUT THE TEST}

Test $\mathrm{H} 2$ subjected a fresh LMFBR fuel pin to a moderately-fast transient overpower excursion sufficient to cause pin failure. (It was nearly identical to test E4 which used a faster overpower transient.) Upward in-pin fuel motion may have begun as early as $100 \mathrm{~ms}$ before cladding failure. Mild sodium boiling existed for 25 to $50 \mathrm{~ms}$ before failure. The cladding failed $0.21 \mathrm{~s}$ after peak power (when the power level was only $\sim 30 \%$ of peak power, $\sim 90 \%$ of the transient energy had been generated, and $1.5 \mathrm{~kJ} / \mathrm{g}$ had been generated in the test fuel). At failure, a little fuel was prompty released, the inlet flow reversed, and an outlet flow spike occurred. The flow channel at the outlet started to become blocked by relocated fuel and cladding within $\sim 10 \mathrm{~ms}$ after cladding failure and was essentially completely blocked by $\sim 50 \mathrm{~ms}$. A major event occurred $200 \mathrm{~ms}$ after cladding failure, involving redistribution of much fuel and steel. A large fraction of the fuel moved above the original fuel region. There was no evidence of massive fuel movement toward the inlet, and no energetic fuel-codant interactions occurred. Because the radial power profile in the fuel pin was strongly peaked toward the outer periphery, fuel melting began within $0.5 \mathrm{~mm}$ from the fuel outer surface and then moved inward. Thermal equilibrium conditions in the test-fuel pin were being approached when the pin failed; hence the test may be considered more characteristic of a scram-protected accident than an unprotected accident.

Posttest examination revealed that the flowtube wall was penetrated near the top of the fuel column, allowing a large (2.5 times) increase in available volume for sodium to move into laterally. Little molten fuel or steel had entered that space, though. A large fraction of the cladding had been destroyed, and fuel had extensively dispersed out to the flowtube wall but mostly remaining axially within the original fuel region. The inside surface of the flowtube was extensively coated with fuel particles. A complete flow blockage had formed just above the original fuel top. The top of the fuel pin had moved upward $\sim 4 \mathrm{~cm}$. 


\title{
Summary of TREAT Test H3
}

\author{
Performed 29 June 1972
}

Transient No. 1456

\section{PRE-TEST SAMPLE CHARACTERIZATION}

\begin{tabular}{|l|l|}
\hline \multicolumn{2}{|c|}{ FUEL } \\
\hline Form & Fast reactor U-Pu oxide \\
\hline Composition & $\begin{array}{l}75 \mathrm{wt} \% \mathrm{UO}_{2}-25 \mathrm{wt} \% \mathrm{PuO}_{2} \\
65 \% \text { enriched } \mathrm{U} \quad \text { (central pin); } \\
\mathrm{O} / \mathrm{M}=1.97\end{array}$ \\
\hline Geometry & Solid, dished pellets \\
\hline Fissile height & $34.2 \mathrm{~cm}$ \\
\hline $\begin{array}{l}\text { Smear } \\
\text { density }\end{array}$ & $88.9 \%$ of theoretical \\
\hline
\end{tabular}

\begin{tabular}{|l|l|}
\hline \multicolumn{2}{|c|}{ CLADDING } \\
\hline Material & Type 316 stainless steel \\
\hline OD & $5.84 \mathrm{~mm}$ \\
\hline Thickness & $0.38 \mathrm{~mm}$ \\
\hline
\end{tabular}

\begin{tabular}{|l|l|}
\hline \multicolumn{2}{|l|}{ PRE-IRRADIATION CHARACTERISTICS } \\
\hline BU (peak axial) & $\sim 3.5$ at\% \\
\hline Peak linear power & $35 \mathrm{~kW} / \mathrm{m}$ \\
\hline $\begin{array}{l}\text { Peak internal clad } \\
\text { temperature }\end{array}$ & (not available) \\
\hline $\begin{array}{l}\text { Fluence } \\
\text { (spectrum) }\end{array}$ & $\begin{array}{l}\sim 3.2 \times 10^{22} \mathrm{n} / \mathrm{cm}^{2} \\
\text { Fast (EBR-II) spectrum }\end{array}$ \\
\hline
\end{tabular}

\section{OTHER FUEL SAMPLE INFORMATION}

FFTF-type fuel pins, one preirradiated at intermediatepower, the others unirradiated. The fuel column was bounded above and below by $1.3 \mathrm{~cm}$ of $\mathrm{UO}_{2}$ insulator pellets. A $15-\mathrm{cm}$ long Inconel reflector was above the upper insulator pellets. Fuel mass $=68 \mathrm{~g} / \mathrm{pin}$.

\begin{tabular}{|c|c|}
\hline \multicolumn{2}{|c|}{ TEST CONDITIONS, MEASUREMENTS, \& ANALYSES } \\
\hline $\begin{array}{l}\text { Coolant } \\
\text { environment }\end{array}$ & Flowing sodium at $4 \mathrm{~m} / \mathrm{s}\left(740 \mathrm{~cm}^{3} / \mathrm{s}\right)$ and $375{ }^{\circ} \mathrm{C}$ \\
\hline $\begin{array}{l}\text { Transient } \\
\text { conditions }\end{array}$ & $\begin{array}{l}\text { Preheat at } 55 \mathrm{~kW} / \mathrm{m} \text { for } 3.0 \mathrm{~s} \text {, followed by a } 0.25 \\
\text { s power increase to } \sim 140 \mathrm{~kW} / \mathrm{m} \text {, holding at that } \\
\text { power for } 0.4 \mathrm{~s} \text {, then scram. }\end{array}$ \\
\hline $\begin{array}{l}\text { Fuel sample } \\
\text { configuration } \\
\text { in the test }\end{array}$ & $\begin{array}{l}\text { 7-pin bundle, pre-irradiated central pin } \\
\text { surrounded by six unirradiated pins }\end{array}$ \\
\hline $\begin{array}{l}\text { Test vehicle \& } \\
\text { experiment } \\
\text { system }\end{array}$ & $\begin{array}{l}\text { Mark-IIA integral sodium loop with } B_{6} \text { Si filtering } \\
\text { and axial flux-shaping collars. Fluted stainless } \\
\text { steel flowtube } 0.9 \mathrm{~mm} \text { thick in good thermal } \\
\text { contact with } 2.2 \mathrm{~mm} \text { thick stainless steel outer } \\
\text { wall. }\end{array}$ \\
\hline $\begin{array}{l}\text { Measurements } \\
\text { /diagnostics } \\
\text { during test }\end{array}$ & $\begin{array}{l}\text { Sodium temperature, flow rate, and pressure. } \\
\text { Flowtube temperatures. Fuel motion using the } \\
\text { hodoscope. }\end{array}$ \\
\hline $\begin{array}{l}\text { Posttest } \\
\text { coolant flow } \\
\text { condition }\end{array}$ & Same as at the beginning of the test. \\
\hline $\begin{array}{l}\text { Posttest } \\
\text { condition of } \\
\text { test sample(s) }\end{array}$ & No cladding failures. No fuel movement. \\
\hline $\begin{array}{l}\text { Posttest } \\
\text { measurements } \\
\text { \& information }\end{array}$ & $\begin{array}{l}\text { Neutron radiography, gamma scanning fuel } \\
\text { radiochemical measurements. Visual inspection; } \\
\text { Macro- and microphotography. Areal fuel melt } \\
\text { fraction. Cladding bow, strain, and eddy-current } \\
\text { measurement. Laser gas sampling of the } \\
\text { central-pin fuel. }\end{array}$ \\
\hline $\begin{array}{l}\text { Posttest } \\
\text { analyses }\end{array}$ & Thermal-hydraulic, cladding mechanics \\
\hline
\end{tabular}

\section{KEY SUMMARY INFORMATION ABOUT THE TEST}

Test $\mathrm{H} 3$ was the first transient overpower failure-threshold experiment in TREAT that used irradiated fuel and also employed a TREAT transient that was shaped to produce test-fuel temperature distributions typical of steady state prior to the overpower excursion. The overpower ramp simulated a 0.5-1 \$/s reactivity ramp in FFTF/FTR. There were no pressure or flow events during the test, and no fuel motion was detected. Posttest examinations revealed no transient-induced cladding strain occurred. No fuel melting or radial swelling occurred in the central pin, but calculations indicated the peak fuel temperature was just short of the solidus. Measurements indicate up to $\sim 40 \%$ of the fuel area in the hottest fresh pin exceeded the solidus. Relative to an untested preirradiated sibling pin, no grain growth occurred in the fuel of the preirradiated tested pin due to the transient. 


\section{Summary of TREAT Test H4}

Performed 19 December 1973

Transient No. 1530

\section{PRE-TEST SAMPLE CHARACTERIZATION}

\begin{tabular}{|l|l|}
\hline \multicolumn{2}{|c|}{ FUEL } \\
\hline Form & Fast reactor MOX \\
\hline Composition & $\begin{array}{l}75 \text { wt\% } \mathrm{UO}_{2}-25 \text { wt\% } \mathrm{PuO}_{2} \\
77 \% \text { enriched U (central pin) } \\
\text { O/M=1.97 }\end{array}$ \\
\hline Geometry & $\begin{array}{l}\text { Solid pellets but with full-length } \\
\text { central void after pre-irradiation }\end{array}$ \\
\hline Fissile height & $34.4 \mathrm{~cm}$ \\
\hline $\begin{array}{l}\text { Smear } \\
\text { density }\end{array}$ & $\begin{array}{l}84.6 \% \text { of theoretical (as-fabricated } \\
\text { preirradiated fuel); 87.5\% fresh pins }\end{array}$ \\
\hline
\end{tabular}

\begin{tabular}{|l|l|}
\hline \multicolumn{2}{|c|}{ CLADDING } \\
\hline Material & Type 316 stainless steel, 20\% CW \\
\hline OD & $5.84 \mathrm{~mm}$ \\
\hline Thickness & $0.38 \mathrm{~mm}$ \\
\hline
\end{tabular}

\begin{tabular}{|l|l|}
\hline \multicolumn{2}{|l|}{ PRE-IRRADIATION CHARACTERISTICS } \\
\hline BU (peak axial) & 4.4 at.\% \\
\hline Peak linear power & $35 \mathrm{~kW} / \mathrm{m}$ \\
\hline $\begin{array}{l}\text { Peak internal clad } \\
\text { temperature }\end{array}$ & $510^{\circ} \mathrm{C}$ \\
\hline $\begin{array}{l}\text { Fluence } \\
\text { (spectrum) }\end{array}$ & $\begin{array}{l}3.78 \times 10^{\circ 2} \mathrm{~cm}^{-2} \\
\text { Fast (EBR-II) spectrum }\end{array}$ \\
\hline
\end{tabular}

\section{OTHER FUEL SAMPLE INFORMATION}

Above the preirradiated fuel was a $17-\mathrm{cm}$ high stack of $\mathrm{UO}_{2}$ pellets, then a $\sim 13 \mathrm{~cm}$ Inconel rod. Above the fresh fuel was a $\sim 1 \mathrm{~cm}$ stack of $\mathrm{UO}_{2}$ pellets, then a $\sim 15 \mathrm{~cm}$ Inconel rod. All pins were wire-wrapped. Fuel mass $64.3 \mathrm{~g} / \mathrm{pin}$

\begin{tabular}{|l|l|}
\hline \multicolumn{2}{|c|}{ TEST CONDITIONS, MEASUREMENTS, \& ANALYSES } \\
\hline $\begin{array}{l}\text { Coolant } \\
\text { environment }\end{array}$ & $\begin{array}{l}\text { Flowing sodium at } 3.5 \mathrm{~m} / \mathrm{s}(730 \mathrm{~cm} / \mathrm{s}) \text { and } 370 \\
{ }^{\circ} \mathrm{C}\end{array}$ \\
\hline $\begin{array}{l}\text { Transient } \\
\text { conditions }\end{array}$ & $\begin{array}{l}\text { Preheat at } 70 \mathrm{~kW} / \mathrm{m} \text { (235 MW TREAT power) for } \\
2.5 \mathrm{~s} \text {, followed by a power rise on a 0.96-s } \\
\text { period reaching a peak of } 654 \text { MW TREAT } \\
\text { power, with 1.06 s full-width-at-half-maximum. }\end{array}$ \\
\hline $\begin{array}{l}\text { Fuel sample } \\
\text { configuration } \\
\text { in the test }\end{array}$ & $\begin{array}{l}\text { 7-pin bundle, pre-irradiated central pin } \\
\text { surrounded by six unirradiated pins }\end{array}$ \\
\hline $\begin{array}{l}\text { Test vehicle \& } \\
\text { experiment } \\
\text { system }\end{array}$ & $\begin{array}{l}\text { Mark-IIA integral sodium loop with B } \text { and filtering } \\
\text { steel flowtube } 0.9 \text { mm thick surrounded by a } \\
\text { circular 0.9 mm stainless steel outer tube with a } \\
2.4 \text { mm annular helium gap in between }\end{array}$ \\
\hline $\begin{array}{l}\text { Measurements } \\
\text { /diagnostics } \\
\text { during test }\end{array}$ & $\begin{array}{l}\text { Sodium flow rate, pressure, and temperature. } \\
\text { Flowtube temperature. Fuel motion (by } \\
\text { hodoscope) }\end{array}$ \\
\hline $\begin{array}{l}\text { Posttest } \\
\text { coolant flow } \\
\text { condition }\end{array}$ & 10\% of the initial flow rate. \\
\hline $\begin{array}{l}\text { Posttest } \\
\text { condition of } \\
\text { test sample(s) }\end{array}$ & $\begin{array}{l}\text { Gross disruption of all seven fuel pins over the } \\
\text { initial fuel region. }\end{array}$ \\
\hline $\begin{array}{l}\text { Posttest } \\
\text { measurements } \\
\text { \& information }\end{array}$ & Neutron radiography \\
\hline $\begin{array}{l}\text { Posttest } \\
\text { analyses }\end{array}$ & Thermal-hydraulic \\
\hline
\end{tabular}

\section{KEY SUMMARY INFORMATION ABOUT THE TEST}

In test $\mathrm{H} 4$, a preirradiated fuel pin containing a nearly axially-uniform distribution of high-power-structure fuel was tested to failure under conditions representative of a hypothetical $50 c$ unprotected overpower transient in FFTF/FTR. Six fresh pins surrounding the preirradiated pin helped to simulate the local thermal-hydraulic environment in a subassembly of pins. Pin failure occurred at $580 \mathrm{MW}(\sim 170$ $\mathrm{kW} / \mathrm{m}$ in the test fuel) as the power was rising and when the sodium was still well below boiling. The thermocouple data suggested failure was near the fuel midplane. The flowtube was breached within $50 \mathrm{~ms}$ after cladding failure, and the sodium flow into the fuel region began decreasing. At $\sim 0.30 \mathrm{~s}$ after cladding failure, the inlet flow reversed, and $20-25 \mathrm{~g}$ of fuel (about one-third of the inventory in a pin) moved upward from the middle third of the fuel region, with about $10 \mathrm{~g}$ moving to the top of the original fuel region. About $50 \mathrm{~ms}$ later, $30-35 \mathrm{~g}$ of fuel was driven downward from the upper half of the fuel region to the lower half, and $70 \mathrm{~ms}$ later fuel was dispersed upward from the bottom half of the fuel region. Several inlet sodium reentry-expulsion events followed. Due to earlier-than-predicted cladding failure and with no automatic reactor shutdown upon cladding failure, post-failure fission energy generation ( $25 \%$ of the burst energy) was greater than desired and resulted in the melting and relocation of nearly all of the fuel in the seven pins. Most of the fuel and cladding moved upward, with molten steel moving ahead of the fuel. A mass of molten steel froze just above the top of the original fuel column, partially blocking the coolant channels. Gross upward motion of fuel and steel occurred late in the test and was apparenty stopped by the partial steel blockage, resulting in material containing most of the fuel from the seven pins collecting in a 10-cm-high region extending to just above the original top of the fuel columns and nearly filling the cross section bounded by the tube surrounding the flowtube. There were scattered deposits of fuel (mostly pre-irradiated fuel) on the outside of the cladding tubes adjacent to the pin plenum regions. A dense region of fuel debris formed over the bottom $5 \mathrm{~cm}$ of the original fuel column. Sodium flowposttest may have by-passed the lower blockage through the breached flowtube. The flow tube was significantly melted in the axial region near the fuel midplane. 


\title{
Summary of TREAT Test H5
}

\author{
Performed 5 February 1973
}

Transient No. 1490

PRE-TEST SAMPLE CHARACTERIZATION
\begin{tabular}{|l|l|}
\hline \multicolumn{2}{|c|}{ FUEL } \\
\hline Form & Fast reactor MOX \\
\hline Composition & $\begin{array}{l}75 \text { wt } \% \mathrm{U}_{2}-25 \mathrm{w} \% \mathrm{PuO}_{2} \\
65 \% \text { enriched U (central pin) } \\
\text { O/M=1.97 }\end{array}$ \\
\hline Geometry & Solid pellets \\
\hline Fissile height & $34.1 \mathrm{~cm}$ \\
\hline $\begin{array}{l}\text { Smear } \\
\text { density }\end{array}$ & $88.6 \%$ of theoretical \\
\hline
\end{tabular}

\begin{tabular}{|l|l|}
\hline \multicolumn{2}{|c|}{ CLADDING } \\
\hline Material & Type 316 stainless steel, 20\% CW \\
\hline OD & $5.84 \mathrm{~mm}$ \\
\hline Thickness & $0.38 \mathrm{~mm}$ \\
\hline
\end{tabular}

PRE-IRRADIATION CHARACTERISTICS

\begin{tabular}{|l|l|}
\hline BU (peak axial) & $3.4 \mathrm{at} \%$ \\
\hline Peak linear power & $34 \mathrm{~kW} / \mathrm{m}$ \\
\hline $\begin{array}{l}\text { Peak internal clad } \\
\text { temperature }\end{array}$ & $\sim 550{ }^{\circ} \mathrm{C}$ \\
\hline $\begin{array}{l}\text { Fluence } \\
\text { (spectrum) }\end{array}$ & $\begin{array}{l}\sim 3.2 \times 10^{22} \mathrm{n} / \mathrm{cm}^{2} \\
\text { Fast (EBR-II) spectrum }\end{array}$ \\
\hline
\end{tabular}

\section{OTHER FUEL SAMPLE INFORMATION}

Average fission gas retention in the pre-irradiated fuel was $\sim 52 \%$. Each pin contained $\sim 67.5 \mathrm{~g}$ of fuel

\begin{tabular}{|c|c|}
\hline \multicolumn{2}{|c|}{ TEST CONDITIONS, MEASUREMENTS, \& ANALYSES } \\
\hline $\begin{array}{l}\text { Coolant } \\
\text { environment }\end{array}$ & $\begin{array}{l}\text { Flowing sodium at } \sim 3.3 \mathrm{~m} / \mathrm{s}\left(\sim 600 \mathrm{~cm}^{3} / \mathrm{s}\right) \text { and } \\
350{ }^{\circ} \mathrm{C}\end{array}$ \\
\hline $\begin{array}{l}\text { Transient } \\
\text { conditions }\end{array}$ & $\begin{array}{l}\text { Preheat at } 34 \mathrm{~kW} / \mathrm{m} \text { in the central pin ( } 114 \mathrm{MW} \\
\text { TREAT power) for } 3.0 \mathrm{~s} \text {, followed by a power } \\
\text { rise on } 1.1 \text {-s period with } 1.0 \mathrm{~s} \text { full-width at half } \\
\text { maximum and peak of } 600 \mathrm{MW} \text { TREAT power }\end{array}$ \\
\hline $\begin{array}{l}\text { Fuel sample } \\
\text { configuration } \\
\text { in the test }\end{array}$ & $\begin{array}{l}\text { 7-pin bundle, pre-irradiated central pin } \\
\text { surrounded by six unirradiated pins }\end{array}$ \\
\hline $\begin{array}{l}\text { Test vehicle \& } \\
\text { experiment } \\
\text { system }\end{array}$ & $\begin{array}{l}\text { Mark-IIA integral sodium loop with } \mathrm{B}_{6} \text { Si filtering } \\
\text { and axial flux-shaping collars. Fluted stainless } \\
\text { steel flowtube } 0.9 \mathrm{~mm} \text { thick surrounded by a } \\
\text { circular } 0.9 \mathrm{~mm} \text { stainless steel outer tube with a } \\
2.4 \mathrm{~mm} \text { annular helium gap. }\end{array}$ \\
\hline $\begin{array}{l}\text { Measurements } \\
\text { /diagnostics } \\
\text { during test }\end{array}$ & $\begin{array}{l}\text { Sodium temperatures, pressures, and outtet flow } \\
\text { rate. Flowtube temperatures. Fuel motion using } \\
\text { the hodoscope. }\end{array}$ \\
\hline $\begin{array}{l}\text { Posttest } \\
\text { coolant flow } \\
\text { condition }\end{array}$ & $40 \%$ of the initial flow rate \\
\hline $\begin{array}{l}\text { Posttest } \\
\text { condition of } \\
\text { test sample(s) }\end{array}$ & $\begin{array}{l}\text { Slight, localized failure of the pre-irradiated pin. } \\
\text { Mild failures of the fresh pins with considerable } \\
\text { melting of cladding but little fuel release. }\end{array}$ \\
\hline $\begin{array}{l}\text { Posttest } \\
\text { measurements } \\
\& \text { information }\end{array}$ & $\begin{array}{l}\text { Neutron radiography, macro-examination, } \\
\text { cladding diameters, axial gamma scans, fission } \\
\text { product distribution using electron microprobe }\end{array}$ \\
\hline $\begin{array}{l}\text { Posttest } \\
\text { analyses }\end{array}$ & $\begin{array}{l}\text { Thermal-hydraulic, cladding mechanics, codant } \\
\text { dynamics }\end{array}$ \\
\hline
\end{tabular}

\section{KEY SUMMARY INFORMATION ABOUT THE TEST}

In test $\mathrm{H} 5$, a preirradiated FFTF-type fuel pin containing intermediate-power-structure fuel (equiaxed and columnar grains but essentially no central void at the midplane) was tested to failure under conditions representing a hypothetical $50 c$ unprotected overpower transient in FFTF/FTR. Six fresh pins surrounding the pre-irradiated pin helped to simulate the local thermal-hydraulic environment in a subassembly of pins. (Five of those six pins had been used in the periphery of the seven-pin cluster in TREAT test H3, where they had experienced no measurable cladding plastic strain and at most $40 \%$ of the fuel area above solidus.) Pin failure occurred at $\sim 180 \mathrm{~kW} / \mathrm{m}$ in the pre-irradiated pin, prior to codant boiling. About 60-70 ms thereafter, one or more of the hottest fresh pins failed (at $\sim 260 \mathrm{~kW} / \mathrm{m}$ ), and two small breaches were formed in the flowtube at elevations $x / L=0.7$ and 0.9 . The TREAT power began dropping fast starting 10 ms later. Because only $\sim 15 \%$ of the overpower transient energy was generated after failure, the condition of the pin cluster immediately following pin fail ure was preserved. Little fuel motion occurred during the test. Peak pressure recorded was $1.5 \mathrm{MPa}$.

During disassembly of the hardware, the pins were individually removed from the pin bundle. It was concluded from the posttest examination that the central, preirradiated pin was the first pin to fail. The failure site of that pin was at a fuel elevation $X / L=0.84$ and extended axially only $2 \mathrm{~cm}$, with a short spiral breach (following the spacer wire) extending immediately above. About $1 \mathrm{~g}$ of fuel was lost from the exposed fuel pellets at the failure site. Only $10 \%$ of the fuel cross section at that elevation had exceeded the solidus temperature during the test. At the fuel axial midplane ( $x / L=0.5), 25 \%$ of the fuel area had exceeded the solidus, was very porous, and was surrounded by, but not in close contact with, a shell of unmelted fuel. About 0.2 to $0.4 \%$ cladding strain was measured posttest in that pin. Cladding of the fresh pins melted above the axial midplane, apparently after codant voiding began. No inelastic strain occurred in any of the fresh pin cladding where measurements could be made. The maximum fuel areal fraction above solidus in the hottest fresh pins was $50-60 \%$. 


\title{
Summary of TREAT Test $\mathrm{H} 6$
}

\author{
Performed 10 November 1977
}

Transient No. 1950

\section{PRE-TEST SAMPLE CHARACTERIZATION}

\begin{tabular}{|l|l|}
\hline \multicolumn{2}{|c|}{ FUEL } \\
\hline Form & Fast reactor MOX \\
\hline Composition & $\mathrm{UO}^{2}-25 \% \mathrm{PuO}^{2} ; 65 \%$ enriched U \\
\hline Geometry & Pellet stack \\
\hline Fissile height & $34.3 \mathrm{~cm}$ \\
\hline $\begin{array}{l}\text { Smear } \\
\text { density }\end{array}$ & $85 \%$ of theoretical \\
\hline
\end{tabular}

\begin{tabular}{|l|l|}
\hline \multicolumn{2}{|c|}{ CLADDING } \\
\hline Material & 316 stainless steel $(20 \%$ cold-worked) \\
\hline OD & $5.84 \mathrm{~mm}$ \\
\hline Thickness & $0.38 \mathrm{~mm}$ \\
\hline
\end{tabular}

\section{PRE-IRRADIATION CHARACTERISTICS}

\begin{tabular}{|l|l|}
\hline BU (peak axial) & $\sim 6$ at.\% \\
\hline Peak linear power & $30 \mathrm{~kW} / \mathrm{m}$ \\
\hline $\begin{array}{l}\text { Peak internal clad } \\
\text { temperature }\end{array}$ & Not available. \\
\hline $\begin{array}{l}\text { Fluence } \\
\text { (spectrum) }\end{array}$ & $\begin{array}{l}\text { Fluence not available. } \\
\text { Fast (EBR-II) spectrum }\end{array}$ \\
\hline
\end{tabular}

Fuel mass $\sim 64 \mathrm{~g} / \mathrm{pin}$. Above the fuel column was $17 \mathrm{~cm}$ of $\mathrm{UO}_{2}$ then $13 \mathrm{~cm}$ of stainless steel reflector. Pins were wire-wrapped

\begin{tabular}{|l|l|}
\hline \multicolumn{2}{|c|}{ TEST CONDITIONS, MEASUREMENTS, \& ANALYSES } \\
\hline $\begin{array}{l}\text { Coolant } \\
\text { environment }\end{array}$ & Flowing sodium initially at $\sim 6 \mathrm{~m} / \mathrm{s}$ and $470{ }^{\circ} \mathrm{C}$ \\
\hline $\begin{array}{l}\text { Transient } \\
\text { conditions }\end{array}$ & $\begin{array}{l}\text { Preheat for } 2.25 \mathrm{~s} \text { at } 36 \mathrm{~kW} / \mathrm{m}(\sim 100 \mathrm{MW} \\
\text { TREAT power) followed by a power rise on a } \\
1.65 \mathrm{~s} \text { period peaking at } 685 \mathrm{MW} \text {. Overpower } \\
\text { burst full-width-at-half-maximum was } \sim 1.5 \mathrm{~s} .\end{array}$ \\
\hline $\begin{array}{l}\text { Fuel sample } \\
\text { configuration } \\
\text { in the test }\end{array}$ & 7 -pin bundle of pre-irradiated pins \\
\hline $\begin{array}{l}\text { Test vehicle \& } \\
\text { experiment } \\
\text { system }\end{array}$ & $\begin{array}{l}\text { Mark-IIC integral sodium loop with neutron } \\
\text { filtering and axial-flux-shaping collars; fluted } \\
\text { stainless steel flowtube surrounded by inert-gas } \\
\text { annulus. }\end{array}$ \\
\hline $\begin{array}{l}\text { Measurements } \\
\text { /diagnostics } \\
\text { during test }\end{array}$ & $\begin{array}{l}\text { Sodium and flowtube temperatures, inlet and } \\
\text { outlet pressure, inlet and outlet flow rate, fuel } \\
\text { motion (using the hodoscope) }\end{array}$ \\
\hline $\begin{array}{l}\text { Posttest } \\
\text { coolant flow } \\
\text { condition }\end{array}$ & Complete flow blockage \\
\hline $\begin{array}{l}\text { Posttest } \\
\text { condition of } \\
\text { test sample(s) }\end{array}$ & $\begin{array}{l}\text { Top half of fuel region was highly disrupted. } \\
\text { Bottom half was largely intact. Small amounts of } \\
\text { fuel moved axially far above the original fuel } \\
\text { region. }\end{array}$ \\
\hline $\begin{array}{l}\text { Posttest } \\
\text { measurements } \\
\text { \& information }\end{array}$ & Neutron radiography \\
\hline $\begin{array}{l}\text { Posttest } \\
\text { analyses }\end{array}$ & Thermal-hydraulic \\
\hline
\end{tabular}

\section{KEY SUMMARY INFORMATION ABOUT THE TEST}

Test $\mathrm{H} 6$ subjected the test pins (a low-to-moderate-power structure pre-irradiated pin and six surrounding fresh pins) to a simulated hypothetical 50 \&/s FFTF/FTR unprotected overpower transient. Pin fail ure was first indicated when the TREAT power was $530 \mathrm{MW}$, the pin linear power was $\sim 200 \mathrm{~kW} / \mathrm{m}$, and two-thirds of the overpower burst energy had been generated. The inlet flow rate decreased only about $20 \%$ (too little to trigger TREAT scram), although the outtet flow rate spiked, and about $5 \mathrm{~g}$ of fuel was expelled upward in the flow channel and lodged on the cladding adjacent to the pin reflectors. A significant outlet flow acceleration occurred $0.15 \mathrm{~s}$ later, followed by outlet flow oscillations and a gradual decrease in the inlet flow rate. Some fuel accumulated in a region centered on the fuel midplane, followed by movement of about $3 \mathrm{~g}$ of fuel to above the active fuel region.

About $0.48 \mathrm{~s}$ after initial failure (when $93 \%$ of the overpower burst energy had been generated), a larger event occurred, involving a large, rapid outet flow increase, abrupt stopping of the inlet flow (triggering reactor scram), and a pressure spike of $\sim 12 \mathrm{MPa}$ at the inlet. The flowtube had failed by this time, and sodium was entering the gas space surrounding the flow tube. The event caused considerable fuel motion, with about $3 \%$ of the fuel inventory in the test moving to regions near the upper reflector, about the same amount moving to well below the active fuel zone (probably dropping within the gas annulus), and fuel also moving within the original fuel region.

Posttest examination showed that the lower half of the active fuel column region remained nearly intact. Fuel from the top half of the fuel column region had moved to locations above or below the original fuel region. Some fuel had moved also into the gas annulus surrounding the flowtube. No axial movement of fuel pins (solid cladding containing fuel) was apparent. 


\section{Appendix O: One-page Summaries of EOS-series Tests}

One-page summaries of each of the EOS-series tests are included here. These summaries can also be found in TREXR [6]. 


\section{Summary of TREAT Test EOS-1 \\ Performed 17 February 1977 \\ Transient No. 1885}

\begin{tabular}{l}
\hline \multicolumn{2}{|c|}{ PRE-TEST SAMPLE CHARACTERIZATION } \\
\begin{tabular}{|l|l|}
\hline \multicolumn{2}{|c|}{ FUEL } \\
\hline Form & Fast reactor He-bonded MOX \\
\hline Composition & $\begin{array}{l}75 \% \mathrm{UO}_{2}, 25 \% \mathrm{PuO}_{2} \\
65 \% \text { enr. U in test pin; natural U in } \\
\text { control pin; } \\
\text { O/M ratio }=1.996\end{array}$ \\
\hline Geometry & Solid pellet \\
\hline Fissile height & $10.2 \mathrm{~cm}$ \\
\hline $\begin{array}{l}\text { Smear } \\
\text { density }\end{array}$ & $85 \%$ of theoretical \\
\hline
\end{tabular}
\end{tabular}

\begin{tabular}{|l|l|}
\hline \multicolumn{2}{|c|}{ CLADDING } \\
\hline Material & 316 stainless steel \\
\hline OD & $5.34 \mathrm{~mm}$ test pin; $6.60 \mathrm{~mm}$ control pin \\
\hline Thickness & $0.13 \mathrm{~mm}$ test pin; $0.76 \mathrm{~mm}$ control pin \\
\hline
\end{tabular}

\begin{tabular}{|l|l|}
\hline \multicolumn{2}{|l|}{ PRE-IRRADIATION CHARACTERISTICS } \\
\hline BU (peak axial) & None \\
\hline Peak linear power & (not applicable) \\
\hline $\begin{array}{l}\text { Peak internal clad } \\
\text { temperature }\end{array}$ & (not applicable) \\
\hline $\begin{array}{l}\text { Fluence } \\
\text { (spectrum) }\end{array}$ & (not applicable) \\
\hline
\end{tabular}

\begin{tabular}{l}
\hline OTHER FUEL SAMPLE INFORMATION \\
\hline $\begin{array}{l}\text { Boron nitride pellets were located at each end of the fuel } \\
\text { column. }\end{array}$ \\
\hline
\end{tabular}

\begin{tabular}{|c|c|}
\hline \multicolumn{2}{|c|}{ TEST CONDITIONS, MEASUREMENTS, \& ANALYSES } \\
\hline $\begin{array}{l}\text { Coolant } \\
\text { environment }\end{array}$ & No codant \\
\hline $\begin{array}{l}\text { Transient } \\
\text { conditions }\end{array}$ & $\begin{array}{l}\text { High-intensity, short durationTREAT power } \\
\text { burst, initial period = } 23 \text { ms, full-width-at-half- } \\
\text { maximum }=110 \mathrm{~ms} \text {, generating peak power of } \\
4300 \mathrm{~kW} / \mathrm{m} \text { in the test pin (approximately } 100 \\
\text { times nominal power) and } 935 \mathrm{~kW} / \mathrm{m} \text { in the } \\
\text { contrd pin }\end{array}$ \\
\hline $\begin{array}{l}\text { Fuel sample } \\
\text { configuration } \\
\text { in the test }\end{array}$ & $\begin{array}{l}\text { Each pin in a separate sealed capsule. } \\
\text { Capsules located in tandem vertically }\end{array}$ \\
\hline $\begin{array}{l}\text { Test vehicle } \& \\
\text { experiment } \\
\text { system }\end{array}$ & $\begin{array}{l}\text { Mark-IIC Capsule Containment Assembly } \\
\text { (modification of part of a Mark-IICloop), with } \\
\text { boron thermal-neutron filtering. A 2.3-mm-thick } \\
\text { boron nitride wall (I.D. = } 8.74 \mathrm{~mm} \text { ) surrounded } \\
\text { the test fuel pin. A thicker BN wall surrounded } \\
\text { the contro pin. }\end{array}$ \\
\hline $\begin{array}{l}\text { Measurements } \\
\text { /diagnostics } \\
\text { during test }\end{array}$ & $\begin{array}{l}\text { Pressure, temperature, fuel motion, including a } \\
\text { thermocouple located close to the fuel column }\end{array}$ \\
\hline $\begin{array}{l}\text { Posttest } \\
\text { coolant flow } \\
\text { condition }\end{array}$ & (not applicable) \\
\hline $\begin{array}{l}\text { Posttest } \\
\text { condition of } \\
\text { test sample(s) }\end{array}$ & $\begin{array}{l}\text { Test pin failed, complete fuel dispersal radially } \\
\text { and axially } \\
\text { Control pin did not fail. }\end{array}$ \\
\hline $\begin{array}{l}\text { Posttest } \\
\text { measurements } \\
\text { \& information }\end{array}$ & $\begin{array}{l}\text { Hodoscope scans, gamma radiography, micro- } \\
\text { examination }\end{array}$ \\
\hline $\begin{array}{l}\text { Posttest } \\
\text { analyses }\end{array}$ & Transient fuel behavior \\
\hline
\end{tabular}

\section{KEY SUMMARY INFORMATION ABOUT THE TEST}

Test EOS-1 was conducted to provide experimental evidence of the effect of fission gas on fuel motion during a high-power burst in the absence of sodium codant. It provided baseline data to compare with the effects of a similar transient on pre-irradiate fuels in tests EOS2 and EOS-3. The test pin in EOS-1 was intended to fail. The control pin was designed to survive intact to supply calibration data and transient-induced microstructural changes for fuel behavior code validation. Based on data from the thermocouple located close to the fuel column, it was concluded that the fresh test fuel pin failed at or soon after peak transient power, just when calculations indicated that the test fuel liquidus was reached, when about half of the transient energy had been generated. It was presumed that some fuel vapor pressure was the cause of fuel being driven outward to contact the thermocouple (since no fission gas was present), and it was believed that the observed upward dispersal of the test fuel was caused by stainless steel vapor pressure generated from hot fuel-steel contact. 


\section{Summary of TREAT Test EOS-2 \\ Performed 22 February 1977 \\ Transient No. 1886}

PRE-TEST SAMPLE CHARACTERIZATION
\begin{tabular}{|l|l|}
\hline \multicolumn{2}{|c|}{ FUEL } \\
\hline Form & Fast reactor He-bonded MOX \\
\hline Composition & $\begin{array}{l}75 \% \mathrm{UO}_{2}, 25 \% \mathrm{PuO}_{2} \\
65 \% \text { enr. U in test pin; natural U in } \\
\text { control pin } \\
\text { Initial O/M ratio = 1.996 }\end{array}$ \\
\hline Geometry & Solid pellet \\
\hline Fissile height & $10.2 \mathrm{~cm}$ \\
\hline $\begin{array}{l}\text { Smear } \\
\text { density }\end{array}$ & $85 \%$ of theoretical \\
\hline
\end{tabular}

\begin{tabular}{|l|l|}
\hline \multicolumn{2}{|c|}{ CLADDING } \\
\hline Material & 316 stainless steel, $20 \% \mathrm{CW}$ \\
\hline OD & $5.34 \mathrm{~mm}$ test pin; $6.60 \mathrm{~mm}$ control pin \\
\hline Thickness & $0.13 \mathrm{~mm}$ test pin; $0.76 \mathrm{~mm}$ control pin \\
\hline
\end{tabular}

\begin{tabular}{|l|l|}
\hline PRE-IRRADIATION CHARACTERISTICS \\
\hline BU (peak axial) & $\begin{array}{l}\text { Test pin: 0.85 at\% of HM } \\
\text { Contrd pin: no burnup }\end{array}$ \\
\hline Peak linear power & $\sim 37 \mathrm{~kW} / \mathrm{m}$ \\
\hline $\begin{array}{l}\text { Peak internal clad } \\
\text { temperature }\end{array}$ & Not available \\
\hline $\begin{array}{l}\text { Fluence } \\
\text { (spectrum) }\end{array}$ & $\begin{array}{l}\text { Fluence not available; } \\
\text { Fast (EBR-II) spectrum }\end{array}$ \\
\hline
\end{tabular}

\section{OTHER FUEL SAMPLE INFORMATION}

High-power-structure in pre-irradiated fuel (central void radius $r / R=0.18$, columnar zone outer radius $r / R=0.62$, equiaxed zone outer radius $r / R=0.75$, unrestructured zone outer radius $r / R=1.0$. The test pin was modified after pre-irradiation: the fuel column was shortened, BN pellets were placed at each end of the fuel column, the cladding was resealed at $2 \mathrm{~atm}$. pressure, and the cladding thickness was reduced to $0.13 \mathrm{~mm}$.

\begin{tabular}{|c|c|}
\hline \multicolumn{2}{|c|}{ TEST CONDITIONS, MEASUREMENTS, \& ANALYSES } \\
\hline $\begin{array}{l}\text { Coolant } \\
\text { environment }\end{array}$ & No codant \\
\hline $\begin{array}{l}\text { Transient } \\
\text { conditions }\end{array}$ & $\begin{array}{l}\text { High-intensity, short duration power burst, initial } \\
\text { period =24 ms, full-width-at-half-maximum = } \\
120 \mathrm{~ms} \text {, generating peak power of } 4055 \mathrm{~kW} / \mathrm{m} \text { in } \\
\text { the test pin (approximately } 100 \text { times nominal } \\
\text { power) and } 920 \mathrm{~kW} / \mathrm{m} \text { in the control pin }\end{array}$ \\
\hline $\begin{array}{l}\text { Fuel sample } \\
\text { configuration } \\
\text { in the test }\end{array}$ & $\begin{array}{l}\text { Each pin in a separate sealed capsule. } \\
\text { Capsules located in tandem vertically }\end{array}$ \\
\hline $\begin{array}{l}\text { Test vehicle \& } \\
\text { experiment } \\
\text { system }\end{array}$ & $\begin{array}{l}\text { Mark-IIC Capsule Containment Assembly } \\
\text { (modification of part of Mark-\|Cloop), with boron } \\
\text { thermal-neutron filtering. A 2.3-mm-thick boron } \\
\text { nitride wall (I.D. }=8.74 \mathrm{~mm} \text { ) surrounded the test } \\
\text { fuel pin. (A thicker BN wall surrounded the } \\
\text { contrd pin.) }\end{array}$ \\
\hline $\begin{array}{l}\text { Measurements } \\
\text { /diagnostics } \\
\text { during test }\end{array}$ & $\begin{array}{l}\text { Pressure, temperature, fuel motion, including a } \\
\text { thermocouple located close to the fuel column }\end{array}$ \\
\hline $\begin{array}{l}\text { Posttest } \\
\text { coolant flow } \\
\text { condition }\end{array}$ & (not applicable) \\
\hline $\begin{array}{l}\text { Posttest } \\
\text { condition of } \\
\text { test sample(s) }\end{array}$ & $\begin{array}{l}\text { Test pin failed, complete fuel dispersal radially } \\
\text { and axially } \\
\text { Contrd pin: no cladding failure }\end{array}$ \\
\hline $\begin{array}{l}\text { Posttest } \\
\text { measurements } \\
\text { \& information }\end{array}$ & $\begin{array}{l}\text { Hodoscope scans, gamma radiography, micro- } \\
\text { examination }\end{array}$ \\
\hline $\begin{array}{l}\text { Posttest } \\
\text { analyses }\end{array}$ & Transient fuel behavior \\
\hline
\end{tabular}

\section{KEY SUMMARY INFORMATION ABOUT THE TEST}

Test EOS-2 was conducted to provide experimental evidence of the effect of fission gas on fuel motion of high-power-structure fuel during a fast high-power burst in the absence of sodium coolant, for comparison with results of EOS-3 (on medium-power-structure irradiated fuel) and EOS-1 (on fresh fuel). The test pin was intended to fail, while the control pin was designed to survive intact to supply calibration data and transient-induced microstructural changes for fuel behavior code validation. Loss of intact geometry of the test pin occurred at peak power (when roughly half of the transient energy had been generated), at a fuel specific energy roughly $200 \mathrm{~J} / \mathrm{g}$ lower than at fuel failure in EOS-1 and $\sim 100 \mathrm{~J} / \mathrm{g}$ higher than at failure in test EOS-3. The time lag between the first-detected axial fuel motion and significant axial fuel motion was about $60 \mathrm{~ms}$. Most of the fuel initially moved upward. The upward fuel motion appeared to be more energetic or extensive than in EOS-1. The posttest appearance of the fuel material was like that in EOS-1. Posttest evidence indicated that after fuel movement ended and the fuel solidified, molten steel tended to drain downward.

Pellets from the fresh fuel pin remained in good condition in the test, with no evidence of exterior cracks and no increase in diameter. 


\section{Summary of TREAT Test EOS-3 \\ Performed 20 April 1977 \\ Transient No. 1904}

\section{PRE-TEST SAMPLE CHARACTERIZATION}

\begin{tabular}{|l|l|}
\hline \multicolumn{2}{|c|}{ FUEL } \\
\hline Form & Fast reactor He-bonded MOX \\
\hline Composition & $\begin{array}{l}75 \% \mathrm{UO}_{2}, 25 \% \mathrm{PuO}_{2} \\
93 \% \text { enr. U in test pin; 9\% enr. U in } \\
\text { control pin } \\
\text { Initial O/M ratio = 1.975 }\end{array}$ \\
\hline Geometry & Solid pellet \\
\hline Fissile height & $10.2 \mathrm{~cm}$ \\
\hline $\begin{array}{l}\text { Smear } \\
\text { density }\end{array}$ & $85 \%$ of theoretical \\
\hline
\end{tabular}

\begin{tabular}{|l|l|}
\hline \multicolumn{2}{|c|}{ CLADDING } \\
\hline Material & 304 stainless steel, annealed \\
\hline OD & $5.79 \mathrm{~mm}$ test pin; $7.06 \mathrm{~mm}$ control pin \\
\hline Thickness & $0.13 \mathrm{~mm}$ test pin; $0.76 \mathrm{~mm}$ control pin \\
\hline
\end{tabular}

\begin{tabular}{|l|l|}
\hline \multicolumn{2}{|l|}{ PRE-IRRADIATION CHARACTERISTICS } \\
\hline BU (peak axial) & $\begin{array}{l}\text { Test pin: 0.81 at\% of HM } \\
\text { Contrd pin: no burnup }\end{array}$ \\
\hline Peak linear power & $\sim 27 \mathrm{~kW} / \mathrm{m}$ \\
\hline $\begin{array}{l}\text { Peak internal clad } \\
\text { temperature }\end{array}$ & Not available \\
\hline $\begin{array}{l}\text { Fluence } \\
\text { (spectrum) }\end{array}$ & $\begin{array}{l}\text { Fluence not available; } \\
\text { Fast (EBR-II) spectrum }\end{array}$ \\
\hline
\end{tabular}

\section{OTHER FUEL SAMPLE INFORMATION}

Medium-power-structure in pre-irradiated fuel (no central void or columnar zone, equiaxed zone outer radius $r / R=0.48-0.62$, unrestructured zone outer radius $r / R=1.0$. The test pin was modified after pre-irradiation: the fuel column was shortened, BN pellets were placed at each end of the fuel column, the cladding was resealed at 2 atm pressure, and the cladding thickness was reduced to $0.13 \mathrm{~mm}$

\begin{tabular}{|c|c|}
\hline \multicolumn{2}{|c|}{ TEST CONDITIONS, MEASUREMENTS, \& ANALYSES } \\
\hline $\begin{array}{l}\text { Coolant } \\
\text { environment }\end{array}$ & No codant \\
\hline $\begin{array}{l}\text { Transient } \\
\text { conditions }\end{array}$ & $\begin{array}{l}\text { High-intensity, short duration power burst, initial } \\
\text { period of } 27 \mathrm{~ms} \text {, full-width-at-half-maximum = } \\
120 \mathrm{~ms} \text {, generating peak power of } 4055 \mathrm{~kW} / \mathrm{m} \text { in } \\
\text { the test pin (approximately } 100 \text { times nominal } \\
\text { power) and } 920 \mathrm{~kW} / \mathrm{m} \text { in the control pin }\end{array}$ \\
\hline $\begin{array}{l}\text { Fuel sample } \\
\text { configuration } \\
\text { in the test }\end{array}$ & $\begin{array}{l}\text { Each pin in a separate sealed capsule. } \\
\text { Capsules located in tandem vertically }\end{array}$ \\
\hline $\begin{array}{l}\text { Test vehicle \& } \\
\text { experiment } \\
\text { system }\end{array}$ & $\begin{array}{l}\text { Mark-IIC Capsule Containment Assembly } \\
\text { (modification of part of Mark-\|Cloop) with boron } \\
\text { thermal-neutron filtering. A 2.3-mm-thick boron } \\
\text { nitride wall (I.D. = } 9.14 \mathrm{~mm} \text { ) surrounded the test } \\
\text { fuel pin. A thicker BN wall surrounded the control } \\
\text { pin.) }\end{array}$ \\
\hline $\begin{array}{l}\text { Measurements } \\
\text { /diagnostics } \\
\text { during test }\end{array}$ & $\begin{array}{l}\text { Pressure, temperature, fuel motion, including a } \\
\text { thermocouple located close to the fuel column }\end{array}$ \\
\hline $\begin{array}{l}\text { Posttest } \\
\text { coolant flow } \\
\text { condition }\end{array}$ & (not applicable) \\
\hline $\begin{array}{l}\text { Posttest } \\
\text { condition of } \\
\text { test sample(s) }\end{array}$ & $\begin{array}{l}\text { Test pin failed, complete fuel dispersal radially } \\
\text { and axially, fuel was completely melted and had } \\
\text { slumped downward. } \\
\text { The control pin did not fail. }\end{array}$ \\
\hline $\begin{array}{l}\text { Posttest } \\
\text { measurements } \\
\text { \& information }\end{array}$ & $\begin{array}{l}\text { Hodoscope scans, gamma radiography, gamma } \\
\text { scanning, micro-examination }\end{array}$ \\
\hline $\begin{array}{l}\text { Posttest } \\
\text { analyses }\end{array}$ & Transient fuel behavior \\
\hline
\end{tabular}

\section{KEY SUMMARY INFORMATION ABOUT THE TEST}

Test EOS-3 was conducted to provide experimental evidence of the effect of fission gas on fuel motion of medium-power-structure fuel during a high-power burst in the absence of sodium coolant, for comparison with results of EOS-2 (on high-power-structure irradiated fuel) and EOS-1 (on fresh fuel). The test pin was intended to fail, while the control pin was designed to survive intact to supply calibration data and transient-induced microstructural changes for fuel behavior code validation. Loss of intact geometry of the test pin near peak power (when roughly half of the transient energy had been generated), earlier than in fresh-fuel test EOS- 1 by roughly $300 \mathrm{~J} / \mathrm{g}$ and earlier than in test EOS-2 by $\sim 100 \mathrm{~J} / \mathrm{g}$ It is believed that axial fuel dispersal of the test fuel (generally upward) in EOS-3 was due to fission gas pressure increase. The time lag between first indications of fuel motion and significant fuel motion was about $60 \mathrm{~ms}$. Posttest examination showed that, by the end of the test, all of the fuel had been melted, and most of the cladding had been melted. The molten steel was present as discrete lumps associated with fuel and as a finely-dispersed metallic phase within the fuel. Fuel material was more porous (density about $8.4 \mathrm{~g} / \mathrm{cm}^{3}$ ) than the fuel remains in EOS-1 and EOS-2 (ranging from 9.3 to $10.7 \mathrm{~g} / \mathrm{cm}^{3}$ ).

Pellets from the fresh fuel pin remained in good condition in the test, with no evidence of exterior cracks and no increase in diameter. 


\section{Appendix P: One-page Summaries of F-series Tests}

One-page summaries of each of the F-series tests are included here. These summaries can also be found in TREXR [6]. 


\title{
Summary of TREAT Test F1
}

\author{
Performed 21 November 1974
}

Transient No. 1609

\section{PRE-TEST SAMPLE CHARACTERIZATION}

\begin{tabular}{|l|l|}
\hline \multicolumn{2}{|c|}{ FUEL } \\
\hline Form & Fast reactor gas-bonded $\mathrm{MOX}$ \\
\hline Composition & $75 \% \mathrm{UO}_{2}-25 \% \mathrm{PuO}_{2} ; 75 \%$ enr. U \\
\hline Geometry & Pellet stack in cladding \\
\hline Fissile height & $34.3 \mathrm{~cm}$ \\
\hline $\begin{array}{l}\text { Smear } \\
\text { density }\end{array}$ & (not available) \\
\hline
\end{tabular}

\begin{tabular}{|l|l|}
\hline \multicolumn{2}{|c|}{ CLADDING } \\
\hline Material & 316 stainless steel \\
\hline OD & $5.84 \mathrm{~mm}$ \\
\hline Thickness & $0.38 \mathrm{~mm}$ \\
\hline
\end{tabular}

\begin{tabular}{|l|l|}
\hline \multicolumn{2}{|l|}{ PRE-IRRADIATION CHARACTERISTICS } \\
\hline BU (peak axial) & $2.35 \mathrm{at} \%$ \\
\hline Peak linear power & $39.4 \mathrm{~kW} / \mathrm{m}$ \\
\hline $\begin{array}{l}\text { Peak internal clad } \\
\text { temperature }\end{array}$ & (not available) \\
\hline $\begin{array}{l}\text { Fluence } \\
\text { (spectrum) }\end{array}$ & $\begin{array}{l}\text { Fluence not available. } \\
\text { Fast (EBR-II) flux. }\end{array}$ \\
\hline
\end{tabular}

\section{OTHER FUEL SAMPLE INFORMATION}

Pre-irradiated fuel contained a central void $(r / R=0.16$, columnar region to $r / R=0.65$, equiaxed region to $r / R=0.69$, and unrestructured region to $r / R=1.00$. The wire wrap on the pin was removed pretest. The plenum was vented, re-filled with $X e$ to $0.8 \mathrm{~atm}$ absolute pressure, and re-sealed before the test. About $37 \%$ of the fission gas generated during the pre-irradiation was retained in the fuel.

\begin{tabular}{|c|c|}
\hline \multicolumn{2}{|c|}{ TEST CONDITIONS, MEASUREMENTS, \& ANALYSES } \\
\hline $\begin{array}{l}\text { Coolant } \\
\text { environment }\end{array}$ & $\begin{array}{l}\text { No codant. Capsule was filled with } \mathrm{Ar}-3 \% \mathrm{He} \text { at } \\
\sim 1 \mathrm{~atm} \text { pressure pretest. }\end{array}$ \\
\hline $\begin{array}{l}\text { Transient } \\
\text { conditions }\end{array}$ & $\begin{array}{l}\text { Steady power (at } ~ 38 \mathrm{~kW} / \mathrm{m} \text { peak-axial in the } \\
\text { test fuel (and } 27 \mathrm{~kW} / \mathrm{m} \text { in the heated wall) during } \\
\text { the } 9.7 \mathrm{~s} \text { of the transient (until fuel motion altered } \\
\text { the power coupling with the TREAT core). } \\
\text { Reactor power remained constant and until } \\
\text { scram. }\end{array}$ \\
\hline $\begin{array}{l}\text { Fuel sample } \\
\text { configuration } \\
\text { in the test }\end{array}$ & Single pin \\
\hline $\begin{array}{l}\text { Test vehicle \& } \\
\text { experiment } \\
\text { system }\end{array}$ & $\begin{array}{l}\text { Dry, sealed capsule, filtered with } \mathrm{B}_{6} \text { Si to harden } \\
\text { the neutron spectrum. Dysprosium collars } \\
\text { provided an EBR-II-like axial fuel power profile. } \\
\text { Surrounding the test pin was tungsten-UO2 tube } \\
\text { (enriched U), } 7.87 \text { mm ID, } 0.76 \text { mm thickness, } \\
\text { wrapped in } 0.05 \text {-mm-thick tungsten foil to } \\
\text { provide a heated wall around the test fuel. } \\
\text { Radially outward was a } 0.5 \text {-mm thick polished } \\
\text { Mo reflector and then a stainless steel heat sink. }\end{array}$ \\
\hline $\begin{array}{l}\text { Measurements } \\
\text { /diagnostics } \\
\text { during test }\end{array}$ & $\begin{array}{l}\text { Thermocouple above and below the test fuel } \\
\text { region; capsule pressure transducer, hodoscope } \\
\text { (fuel motion detection) }\end{array}$ \\
\hline $\begin{array}{l}\text { Posttest } \\
\text { coolant flow } \\
\text { condition }\end{array}$ & (not applicable) \\
\hline $\begin{array}{l}\text { Posttest } \\
\text { condition of } \\
\text { test sample(s) }\end{array}$ & Highly disrupted \\
\hline $\begin{array}{l}\text { Posttest } \\
\text { measurements } \\
\text { \& information }\end{array}$ & $\begin{array}{l}\text { Neutron radiography, gamma scanning, macro- } \\
\text { and microphotography }\end{array}$ \\
\hline $\begin{array}{l}\text { Posttest } \\
\text { analyses }\end{array}$ & Thermal, fuel motion \\
\hline
\end{tabular}

\section{KEY SUMMARY INFORMATION ABOUT THE TEST}

Test F1 investigated the effect of fission gas on fuel motion at design power levels following cladding melting and drainage during a hypothetical LMFBR Ioss-of-flow accident simulation. Extensive fuel swelling apparenty occurred prior to gross axial fuel motion. The hodoscope indicated that fuel-column bending began about $2 \mathrm{~s}$ into the $9.7-\mathrm{s}$ tong transient and lasted for $5 \mathrm{~s}$. Small masses of fuel moved downward during that time period. From $4.4 \mathrm{~s}$ to $6.1 \mathrm{~s}$ into the transient, the entire fuel column expanded upward $\sim 1.5 \mathrm{~cm}$. Significant slumping of fuel involving $10 \mathrm{~g}$ of fuel from the midplane region began $12.7 \mathrm{~s}$ into the transient (1.4 $\mathrm{s}$ before reactor scram). Posttest examination showed that the heated wall contained the test fuel. The cladding had melted over the entire length of the fuel column. A single fuel pellet had moved to just above the top of the original fuel region. Otherwise no fuel moved beyond the ends of the original fuel region. In the upper and lower $10 \%$ of the fuel column (elevations where pre-irradiation retained fission gas concentration was highest), the posttest condition of the fuel pellets showed radial swelling that varied gradually from litte swelling at the colder end to swelling at the hotter end extending radially (up to 1.5 times the initial radius) to the heated wall. Most of the upper half of the original axial fuel region $(x / L=0.45-0.89$ ) was voided of fuel. The region $x / L=0.09-0.44$ was filled with once-molten fuel which had flowed down to the top of the partially-melted swelled pellets at the bottom. A large ingot of melted cladding was found near the bottom of the original fue region. Metallic phases were present in the once-molten fuel. 


\title{
Summary of TREAT Test F2
}

\author{
Performed 22 November 1974
}

Transient No. 1610

\section{PRE-TEST SAMPLE CHARACTERIZATION}

\begin{tabular}{|l|l|}
\hline \multicolumn{2}{|c|}{ FUEL } \\
\hline Form & Fast reactor gas-bonded $\mathrm{MOX}$ \\
\hline Composition & $75 \% \mathrm{UO}_{2}-25 \% \mathrm{PuO}_{2} ; 80 \%$ enr. U \\
\hline Geometry & Pellet stack in cladding \\
\hline Fissile height & $34.3 \mathrm{~cm}$ \\
\hline $\begin{array}{l}\text { Smear } \\
\text { density }\end{array}$ & (not available) \\
\hline
\end{tabular}

\begin{tabular}{|l|l|}
\hline \multicolumn{2}{|c|}{ CLADDING } \\
\hline Material & 316 stainless steel \\
\hline OD & $5.84 \mathrm{~mm}$ \\
\hline Thickness & $0.38 \mathrm{~mm}$ \\
\hline
\end{tabular}

\begin{tabular}{|l|l|}
\hline \multicolumn{2}{|l|}{ PRE-IRRADIATION CHARACTERISTICS } \\
\hline BU (peak axial) & 0.35 at\% \\
\hline Peak linear power & $38 \mathrm{~kW} / \mathrm{m}$ \\
\hline $\begin{array}{l}\text { Peak internal clad } \\
\text { temperature }\end{array}$ & (not available) \\
\hline $\begin{array}{l}\text { Fluence } \\
\text { (spectrum) }\end{array}$ & $\begin{array}{l}\text { Fluence not available. } \\
\text { Fast (EBR-II) flux }\end{array}$ \\
\hline
\end{tabular}

\section{OTHER FUEL SAMPLE INFORMATION}

Pre-irradiated fuel contained a central void $(r / R=0.26$, columnar region to $r / R=0.81$, equiaxed region to $r / R=0.87$, and unrestructured region to $r / R=1.00$. The wire wrap on the pin was removed pretest.

\begin{tabular}{|c|c|}
\hline \multicolumn{2}{|c|}{ TEST CONDITIONS, MEASUREMENTS, \& ANALYSES } \\
\hline $\begin{array}{l}\text { Coolant } \\
\text { environment }\end{array}$ & $\begin{array}{l}\text { No codant. Capsule was filled with } \mathrm{Ar}-3 \% \mathrm{He} \text { at } \\
\sim 1 \mathrm{~atm} \text { pressure pretest. }\end{array}$ \\
\hline $\begin{array}{l}\text { Transient } \\
\text { conditions }\end{array}$ & $\begin{array}{l}\text { Steady power preheat for } 6.5 \mathrm{~s} \text { at } 41 \mathrm{~kW} / \mathrm{m} \text { in } \\
\text { the test fuel }(28 \mathrm{~kW} / \mathrm{m} \text { in the heated wall), then a } \\
\sim 450 \mathrm{~ms} \text { full-width-at-half-maximum power burst } \\
\text { to } \sim 11 \text { times the preheat level. }\end{array}$ \\
\hline $\begin{array}{l}\text { Fuel sample } \\
\text { configuration } \\
\text { in the test }\end{array}$ & Single pin \\
\hline $\begin{array}{l}\text { Test vehicle \& } \\
\text { experiment } \\
\text { system }\end{array}$ & 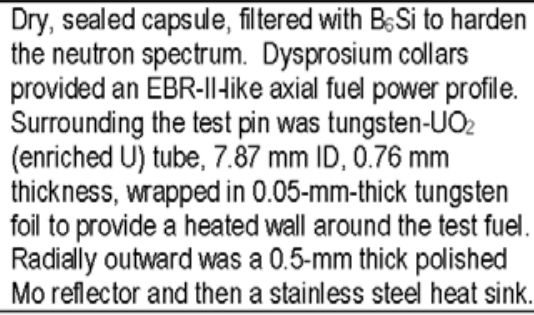 \\
\hline $\begin{array}{l}\text { Measurements } \\
\text { /diagnostics } \\
\text { during test }\end{array}$ & $\begin{array}{l}\text { Thermocouple above and below the test fuel } \\
\text { region; capsule pressure transducer, hodoscope } \\
\text { (fuel motion detection) }\end{array}$ \\
\hline $\begin{array}{l}\text { Posttest } \\
\text { coolant flow } \\
\text { condition }\end{array}$ & (not applicable) \\
\hline $\begin{array}{l}\text { Posttest } \\
\text { condition of } \\
\text { test sample(s) }\end{array}$ & Highly disrupted \\
\hline $\begin{array}{l}\text { Posttest } \\
\text { measurements } \\
\text { \& information }\end{array}$ & $\begin{array}{l}\text { Neutron radiography, gamma scanning, macro- } \\
\text { and microphotography }\end{array}$ \\
\hline $\begin{array}{l}\text { Posttest } \\
\text { analyses }\end{array}$ & Thermal, fuel motion \\
\hline
\end{tabular}

\section{KEY SUMMARY INFORMATION ABOUT THE TEST}

Test F2 investigated the effect of fission gas on fuel motion resulting from a power burst following cladding melting and drainage during a hypothetical LMFBR Ioss-of-flow accident simulation. Just before the power burst began, the hodoscope data indicated that the fuel column was still erect, and the lower thermocouple indicated a substantial amount of molten steel (confirmed posttest) had drained to the bottom of the initial fuel region. The major fuel motion event was upward motion of the top $\sim 10 \mathrm{~cm}$ of the fuel column and occurred when about one-third of the TREAT burst energy had been generated. A second, upward fuel motion event occurred 0.14 slater (around peak reactor power) involving an additional 5 to $8 \mathrm{~cm}$ of the fuel column. During the following $0.7 \mathrm{~s}$, much of the fuel that had moved upward fell back down and accumulated in the region around the bottom half of the pin. Posttest it was determined that fuel was located over a 16-cm-high region extending as high as $19 \mathrm{~cm}$ above the original top of the fuel column, in which was a $10-\mathrm{cm}$-high annular region filled with once-molten fuel. Progressively below that mass were a 14-cm-high region $(x / L=0.7-1.1)$ voided of fuel, a 10-cm-high region $(x / L=0.4-$ 0.7) partially voided of fuel, a 9-cm-high region ( $\mathrm{x} / \mathrm{L}=0.1-0.4)$ region of once-molten fuel extending radially outward to the heated wall, and a 3 -cm-high region $(x / L=0-0.1)$ containing some fuel. Below the original fuel region was a large mass of solidified cladding steel. At an unknown time during the burst, the heated wall had deformed along almost the entire length of the original fuel region, its $\mathrm{UO}_{2}$ phase had melted and mostly relocated (thereby becoming indistinguishable from fuel), and a considerable amount of test fuel had leaked radially through the wall. 


\section{Summary of TREAT Test F3 \\ Performed 3 July 1980}

Transient No. 2216

\section{PRE-TEST SAMPLE CHARACTERIZATION}

\begin{tabular}{|l|l|}
\hline \multicolumn{2}{|c|}{ FUEL } \\
\hline Form & Fast reactor $\mathrm{MOX}$ \\
\hline Composition & $\begin{array}{l}75 \% \mathrm{UO}_{2}-25 \% \mathrm{PuO}_{2}, 45 \% \text { enr. U } \\
\mathrm{O}^{\mathrm{M}}=1.97+1-0.02\end{array}$ \\
\hline Geometry & Solid-pellet stack in cladding \\
\hline Fissile height & $5.1 \mathrm{~cm}$ \\
\hline $\begin{array}{l}\text { Smear } \\
\text { density }\end{array}$ & $88 \%$ of theoretical \\
\hline
\end{tabular}

\begin{tabular}{|l|l|}
\hline \multicolumn{2}{|c|}{ CLADDING } \\
\hline Material & 316 stainless steel \\
\hline OD & $6.35 \mathrm{~mm}$ \\
\hline Thickness & $0.41 \mathrm{~mm}$ \\
\hline
\end{tabular}

\begin{tabular}{|l|l|}
\hline \multicolumn{2}{|l|}{ PRE-IRRADIATION CHARACTERISTICS } \\
\hline BU (peak axial) & 9 at\% \\
\hline Peak linear power & $26.2-29.5 \mathrm{~kW} / \mathrm{m}$ \\
\hline $\begin{array}{l}\text { Peak internal clad } \\
\text { temperature }\end{array}$ & (not available) \\
\hline $\begin{array}{l}\text { Fluence } \\
\text { (spectrum) }\end{array}$ & $\begin{array}{l}\text { Fluence not available. } \\
\text { Fast (EBR-II) spectrum. }\end{array}$ \\
\hline
\end{tabular}

\section{OT HER FUEL SAMPLE INFORMATION}

The fuel pin was made from an $8.2-\mathrm{cm}$ length of the $34.3 \mathrm{~cm}$ fuel region of a pre-irradiated pin. $1.6 \mathrm{~cm}$ of fuel at each cut end was removed, tantalum pellets were placed against the ends of the remaining fuel stack, and end plugs were welded on. The $10-11 \mathrm{~g}$ of fuel (and adjacent cladding) in the finished pin was from an axial location $x / L=0.67-0.83$ along the original fuel column. The fued sample likely had large equiaxed grains at the center of the fuel column.

\begin{tabular}{|l|l|}
\hline \multicolumn{2}{|c|}{ TEST CONDITIONS, MEASUREMENTS, \& ANALYSES } \\
\hline $\begin{array}{l}\text { Coolant } \\
\text { environment }\end{array}$ & No codant \\
\hline $\begin{array}{l}\text { Transient } \\
\text { conditions }\end{array}$ & $\begin{array}{l}\text { Steady TREAT power generating (prior to test- } \\
\text { fuel relocation) } 82 \text { kW/m in the test fuel. }\end{array}$ \\
\hline $\begin{array}{l}\text { Fuel sample } \\
\text { configuration } \\
\text { in the test }\end{array}$ & Single pin \\
\hline $\begin{array}{l}\text { Test vehicle \& } \\
\text { experiment } \\
\text { system }\end{array}$ & $\begin{array}{l}\text { Dry capsule with a window adjacent to the fuel } \\
\text { transient. Included as CdS thermal neutron } \\
\text { filter. }\end{array}$ \\
\hline $\begin{array}{l}\text { Measurements } \\
\text { /diagnostics } \\
\text { during test }\end{array}$ & $\begin{array}{l}\text { High speed photography at 2000 frames/s; } \\
\text { fast-neutron hodoscope }\end{array}$ \\
\hline $\begin{array}{l}\text { Posttest } \\
\text { coolant flow } \\
\text { condition }\end{array}$ & (not applicable) \\
\hline $\begin{array}{l}\text { Posttest } \\
\text { condition of } \\
\text { test sample(s) }\end{array}$ & Highly disrupted \\
\hline $\begin{array}{l}\text { Posttest } \\
\text { measurements } \\
\text { \& information }\end{array}$ & Neutron radiography, macro-examination \\
\hline $\begin{array}{l}\text { Posttest } \\
\text { analyses }\end{array}$ & Thermal \\
\hline
\end{tabular}

\footnotetext{
KEY SUMMARY INFORMATION ABOUT THE TEST

Test F3 was performed to determine the timing and mode of disruption of fuel pins in the lead power-to-flow subassemblies during an unprotected loss-of-flow situation in an LMFBR with a moderately positive sodium void reactivity. The fuel power level was about 2.7 times nominal power in lead power-to-flow subassemblies in CRBR. Fuel failure occurred when approximately $870 \mathrm{~J} / \mathrm{g}$ of energy had been generated in the test fuel. The initial fuel motion caused fuel to move laterally $25 \mathrm{~mm}$ to the mirror in about $1.5 \mathrm{~ms}$. Calculations indicate the fuel was not molten when the cladding first failed. Posttest examination showed that the mid-section of the fuel region was destroyed, the upper segment of the pin had moved down about $4.5 \mathrm{~cm}$ and was tilted, the fuel had partially melted, and there was some fuel dispersal. There was no fuel in either the upper or lower fuel pin segment. The bottom $0.5 \mathrm{~mm}$ of the upper tantalum pellet showed signs of having been heat-affected by the transient.
} 


\section{Summary of TREAT Test F4 \\ Performed 12 June 1980 \\ Transient No. 2214}

\section{PRE-TEST SAMPLE CHARACTERIZATION}

\begin{tabular}{|l|l|}
\hline \multicolumn{2}{|c|}{ FUEL } \\
\hline Form & Fast reactor $\mathrm{MOX}$ \\
\hline Composition & $\begin{array}{l}75 \% \mathrm{UO}_{2}-25 \% \mathrm{PuO}_{2}, 45 \% \text { enr. U } \\
\mathrm{O} / \mathrm{M}=1.97+1-0.02 \cup_{2}-\mathrm{PuO}_{2}\end{array}$ \\
\hline Geometry & Solid-pellet stack in cladding \\
\hline Fissile height & $5.1 \mathrm{~cm}$ \\
\hline $\begin{array}{l}\text { Smear } \\
\text { density }\end{array}$ & $88 \%$ of theoretical \\
\hline
\end{tabular}

\begin{tabular}{|l|l|}
\hline \multicolumn{2}{|c|}{ CLADDING } \\
\hline Material & 316 stainless steel \\
\hline OD & $6.35 \mathrm{~mm}$ \\
\hline Thickness & $0.41 \mathrm{~mm}$ \\
\hline
\end{tabular}

\begin{tabular}{|l|l|}
\hline \multicolumn{2}{|l|}{ PRE-IRRADIATION CHARACTERISTICS } \\
\hline BU (peak axial) & 9 at\% \\
\hline Peak linear power & $26.2-29.5 \mathrm{~kW} / \mathrm{m}$ \\
\hline $\begin{array}{l}\text { Peak internal clad } \\
\text { temperature }\end{array}$ & (not available) \\
\hline $\begin{array}{l}\text { Fluence } \\
\text { (spectrum) }\end{array}$ & $\begin{array}{l}\text { Fluence not available. } \\
\text { Fast (EBR-II) spectrum. }\end{array}$ \\
\hline
\end{tabular}

\section{OT HER FUEL SAMPLE INFORMATION}

The fuel pin was made from an $8.2-\mathrm{cm}$ length of the $34.3 \mathrm{~cm}$ fuel region of a pre-irradiated pin. $1.6 \mathrm{~cm}$ of fuel at each cut end was removed, tantalum pellets were placed against the ends of the remaining fuel stack, and end plugs were welded on. The 10-11 g of fuel (and adjacent cladding) in the finished pin was from an axial location $x / L=0.67-0.83$ along the original fuel column. The fued sample likely had large equiaxed grains at the center of the fuel column.

\begin{tabular}{|l|l|}
\hline \multicolumn{2}{|c|}{ TEST CONDITIONS, MEASUREMENTS, \& ANALYSES } \\
\hline $\begin{array}{l}\text { Coolant } \\
\text { environment }\end{array}$ & No codant \\
\hline $\begin{array}{l}\text { Transient } \\
\text { conditions }\end{array}$ & $\begin{array}{l}\text { Steady TREAT power generating (prior to test- } \\
\text { fuel relocation) 216 kW/m in the test fuel. }\end{array}$ \\
\hline $\begin{array}{l}\text { Fuel sample } \\
\text { configuration } \\
\text { in the test }\end{array}$ & Single pin \\
\hline $\begin{array}{l}\text { Test vehicle \& } \\
\text { experiment } \\
\text { system }\end{array}$ & $\begin{array}{l}\text { Dry capsule with a window adjacent to the fuel } \\
\text { column to allow optical diagnostics during the } \\
\text { transient. Included a CdS neutron filter. }\end{array}$ \\
\hline $\begin{array}{l}\text { Measurements } \\
\text { /diagnostics } \\
\text { during test }\end{array}$ & $\begin{array}{l}\text { High speed photography at 2000 frames/s; } \\
\text { fast-neutron hodoscope }\end{array}$ \\
\hline $\begin{array}{l}\text { Posttest } \\
\text { coolant flow } \\
\text { condition }\end{array}$ & (not applicable) \\
\hline $\begin{array}{l}\text { Posttest } \\
\text { condition of } \\
\text { test sample(s) }\end{array}$ & Highly disrupted \\
\hline $\begin{array}{l}\text { Posttest } \\
\text { measurements } \\
\& \text { information }\end{array}$ & Neutron radiography, maxro-examination \\
\hline $\begin{array}{l}\text { Posttest } \\
\text { analyses }\end{array}$ & Thermal \\
\hline
\end{tabular}

\section{KEY SUMMARY INFORMATION ABOUT THE TEST}

Test F4 was performed to determine the timing and mode of disruption of fuel pins in the lead power-to-flow subassemblies during an unprotected loss-of-flow situation in an LMFBR with a moderately positive sodium void reactivity. The fuel power level was about seven times nominal power in lead power-to-flow subassemblies in CRBR. Fuel failure occurred when approximately $810 \mathrm{~J} / \mathrm{g}$ of energy had been generated in the test fuel. Calculations indicate that the fuel was not molten when the cladding first failed, and the cladding may not have been molten either. Posttest examination showed that the mid-section of the fuel region was destroyed, the upper part of the pin had moved down about $2 \mathrm{~cm}$, the fuel had partially melted, and there was some fuel dispersal. The only fuel remaining inside intact cladding was a small amount of apparently unmelted fuel located against the upper tantalum pellet which was still in place. Neither the upper nor the lower tantalum pellet showed any evidence of having been affected by the transient. 


\section{Summary of TREAT Test F6 \\ Performed 9 April 1984 \\ Transient No. 2554}

\section{PRE-TEST SAMPLE CHARACTERIZATION}

\begin{tabular}{|l|l|}
\hline \multicolumn{2}{|c|}{ FUEL } \\
\hline Form & Fast reactor $\mathrm{MOX}$ \\
\hline Composition & $\begin{array}{l}75 \% \mathrm{UO}_{2}-25 \% \mathrm{PuO}_{2}, 45 \% \text { enr. U } \\
\mathrm{O} / \mathrm{M}=1.97+1-0.02 \mathrm{UO}_{2}-\mathrm{PuO}_{2}\end{array}$ \\
\hline Geometry & Pellet stack in cladding \\
\hline Fissile height & $5.1 \mathrm{~cm}$ \\
\hline $\begin{array}{l}\text { Smear } \\
\text { density }\end{array}$ & $88 \%$ of theoretical \\
\hline
\end{tabular}

\begin{tabular}{|l|l|}
\hline \multicolumn{2}{|c|}{ CLADDING } \\
\hline Material & 316 stainless steel \\
\hline OD & $6.35 \mathrm{~mm}$ \\
\hline Thickness & $0.41 \mathrm{~mm}$ \\
\hline
\end{tabular}

\begin{tabular}{|l|l|}
\hline \multicolumn{2}{|l|}{ PRE-IRRADIATION CHARACTERISTICS } \\
\hline BU (peak axial) & 9 at\% \\
\hline Peak linear power & $29.5 \mathrm{~kW} / \mathrm{m}$ \\
\hline $\begin{array}{l}\text { Peak internal clad } \\
\text { temperature }\end{array}$ & (not available) \\
\hline $\begin{array}{l}\text { Fluence } \\
\text { (spectrum) }\end{array}$ & $\begin{array}{l}\text { Fluence not available. } \\
\text { Fast (EBR-II) spectrum. }\end{array}$ \\
\hline
\end{tabular}

\section{OTHER FUEL SAMPLE INFORMATION}

The fuel sample was made from a segment of a preirradiated fuel pin, probably as in tests F3 and F4.

\begin{tabular}{|l|l|}
\hline \multicolumn{2}{|c|}{ TEST CONDITIONS, MEASUREMENTS, \& ANALYSES } \\
\hline $\begin{array}{l}\text { Coolant } \\
\text { environment }\end{array}$ & No codant \\
\hline $\begin{array}{l}\text { Transient } \\
\text { conditions }\end{array}$ & $\begin{array}{l}\text { Constant TREAT power producing a } \\
\text { temperature rise rate of } ~ 600 \mathrm{~K} / \mathrm{s} \text { in the test fuel, } \\
\text { simulating a reactor power of } 2 \text { to } 4 \text { times } \\
\text { nominal (roughly } 90 \mathrm{~kW} / \mathrm{m} \text { in the test fuel). }\end{array}$ \\
\hline $\begin{array}{l}\text { Fuel sample } \\
\text { configuration } \\
\text { in the test }\end{array}$ & Single pin \\
\hline $\begin{array}{l}\text { Test vehicle \& } \\
\text { experiment } \\
\text { system }\end{array}$ & $\begin{array}{l}\text { Dry capsule similar to the capsule used in tests } \\
\text { the sample. CdS neutron filters were included. }\end{array}$ \\
\hline $\begin{array}{l}\text { Measurements } \\
\text { /diagnostics } \\
\text { during test }\end{array}$ & $\begin{array}{l}\text { High-speed photography at } 8000 \text { frames/s; } \\
\text { fuel motion monitoring using the hodoscope }\end{array}$ \\
\hline $\begin{array}{l}\text { Posttest } \\
\text { coolant flow } \\
\text { condition }\end{array}$ & (not applicable) \\
\hline $\begin{array}{l}\text { Posttest } \\
\text { condition of } \\
\text { test sample(s) }\end{array}$ & Highly disrupted \\
\hline $\begin{array}{l}\text { Posttest } \\
\text { measurements } \\
\text { \& information }\end{array}$ & (not available) \\
\hline $\begin{array}{l}\text { Posttest } \\
\text { analyses }\end{array}$ & (not available) \\
\hline
\end{tabular}

\section{KEY SUMMARY INFORMATION ABOUT THE TEST}

Test F6 investigated the behavior of a short segment of mixed-oxide fuel pin heated through pin disruption in a simulation of the thermal conditions in an LMFBR loss-of-flow accident following sodium voiding. It was intended to repeat test F4 except with an improved optical fuel-motion diagnostics system. A clear sequence of photographs of the failing fuel pin was obtained. An initial examination of the photographs showed a local break or tear in the still-solid cladding and a release of a bright (extremely hot) substance that quickly (within $\sim 1 \mathrm{~ms}$ ) filled the viewing area. 


\section{Appendix Q: One-page Summaries of D-series Tests}

One-page summaries of each of the D-series tests are included here. These summaries can also be found in TREXR [6]. 


\section{Summary of TREAT Test D-1 \\ Performed 22 June 1971 \\ Transient No. 1394}

\section{PRE-TEST SAMPLE CHARACTERIZATION}

\begin{tabular}{|l|l|}
\hline \multicolumn{2}{|c|}{ FUEL } \\
\hline Form & $\begin{array}{l}\mathrm{UO}_{2} \text { pellets helium bonded to } \\
\text { cladding }\end{array}$ \\
\hline Composition & $\begin{array}{l}\mathrm{UO}_{2} ; 26 \% \text { enriched U in central pin } \\
\text { (except } 3.8 \mathrm{~cm} \text { of fuel at midplane } \\
93 \% \text { enriched), 20\% enriched in } \\
\text { outer pins; } \\
\text { O/M = 2.00 }\end{array}$ \\
\hline Geometry & Stack of dished pellets \\
\hline Fissile height & $34.3 \mathrm{~cm}$ \\
\hline $\begin{array}{l}\text { Smear } \\
\text { density }\end{array}$ & $9.2 \mathrm{~g} / \mathrm{cm}^{3}$ \\
\hline
\end{tabular}

\begin{tabular}{|l|l|}
\hline \multicolumn{2}{|c|}{ CLADDING } \\
\hline Material & 316 stainless steel \\
\hline OD & $5.84 \mathrm{~mm}$ \\
\hline Thickness & $0.38 \mathrm{~mm}$ \\
\hline
\end{tabular}

\begin{tabular}{|l|l|}
\hline \multicolumn{2}{|l|}{ PRE-IRRADIATION CHARACTERISTICS } \\
\hline BU (peak axial) & None \\
\hline Peak linear power & (not applicable) \\
\hline $\begin{array}{l}\text { Peak internal clad } \\
\text { temperature }\end{array}$ & (not applicable) \\
\hline $\begin{array}{l}\text { Fluence } \\
\text { (spectrum) }\end{array}$ & (not applicable) \\
\hline
\end{tabular}

\section{OTHER FUEL SAMPLE INFORMATION}

All pins were wire-wrapped.

\begin{tabular}{|l|l|}
\hline \multicolumn{2}{|c|}{ TEST CONDITIONS, MEASUREMENTS, \& ANALYSES } \\
\hline $\begin{array}{l}\text { Coolant } \\
\text { environment }\end{array}$ & $\begin{array}{l}\text { Sodium flow at } 850 \mathrm{~cm}^{3} / \mathrm{s} ; \text { inlet temperature } \\
\text { increasing from } 415{ }^{\circ} \mathrm{C} \text { to } 570 \circ \mathrm{C} \text { during the } \\
\text { transient }\end{array}$ \\
\hline $\begin{array}{l}\text { Transient } \\
\text { conditions }\end{array}$ & $\begin{array}{l}\text { Constant power of } 40 \mathrm{~kW} / \mathrm{m}(82 \mathrm{~kW} / \mathrm{m} \text { in the } \\
\text { over-enriched section) in the central pin for } ~ \\
\text { S. The power generated in the outer pins was } \\
10-20 \% \text { less than in the center pin. }\end{array}$ \\
\hline $\begin{array}{l}\text { Fuel sample } \\
\text { configuration } \\
\text { in the test }\end{array}$ & $\begin{array}{l}\text { Seven-pin bundle, with center pin higher } \\
\text { enriched than edge pins }\end{array}$ \\
\hline $\begin{array}{l}\text { Test vehicle \& } \\
\text { experiment } \\
\text { system }\end{array}$ & $\begin{array}{l}\text { Mark-IIA integral sodium loop; 7-pin test train } \\
\text { with flowing sodium area =1.86 cm² surrounded } \\
\text { by a 0.9-mm-thick stainless steel wall }\end{array}$ \\
\hline $\begin{array}{l}\text { Measurements } \\
\text { /diagnostics } \\
\text { during test }\end{array}$ & $\begin{array}{l}\text { Coolant pressure, flow rate, and temperature; } \\
\text { structure temperatures. }\end{array}$ \\
\hline $\begin{array}{l}\text { Posttest } \\
\text { coolant flow } \\
\text { condition }\end{array}$ & No flow blockage \\
\hline $\begin{array}{l}\text { Posttest } \\
\text { condition of } \\
\text { test sample(s) }\end{array}$ & All pins remained intact. \\
\hline $\begin{array}{l}\text { Posttest } \\
\text { measurements } \\
\text { \& information }\end{array}$ & $\begin{array}{l}\text { Neutron radiography; macro- and micro- } \\
\text { examinations; gamma scanning; } \text { X-radiography; } \\
\text { gas analysis; SEM }\end{array}$ \\
\hline analyses & Thermal-hydraulic and cladding mechanics \\
\hline
\end{tabular}

KEY SUMMARY INFORMATION ABOUT THE TEST

Test D1 was intended to obtain information on the conditions required to achieve the release of a small amount of molten fuel from a failed fresh $\mathrm{UO}_{2}$ pin into flowing sodium and, if failure occurred, to provide information useful in determining the mechanism and cause of the release, the extent of any molten-fuel/coolant interaction, the nature of any flow impediment caused by the fuel release, and whether any damage to adjacent pins tends to propagate additional pin failure. Pin failure did not occur in the test, however. Posttest visual examination revealed no anomalies on the pin exteriors. There was no statistically-significant change in length or bow of any of the pins before their wire wraps were removed, and there was little or no change in cladding diameters. The pellets of the fully-enriched fuel in the central pin were extensively cracked, especially the top pellet which was badly shattered. Melting of the fully-enriched pellets (up to $55 \%$ of the fuel area) created central voids in those pellets. Fractured pellets of $26 \%$ enrichment just below the fully-enriched pellets showed no evidence of melting or central void formation. No melting occurred in any of the edge pins. 


\title{
Summary of TREAT Test D-2
}

\author{
Performed 28 October 1971
}

Transient No. 1414

\section{PRE-TEST SAMPLE CHARACTERIZATION}

\begin{tabular}{|l|l|}
\hline \multicolumn{2}{|c|}{ FUEL } \\
\hline Form & $\begin{array}{l}\mathrm{UO}_{2} \text { pellets, helium bonded to } \\
\text { cladding }\end{array}$ \\
\hline Composition & $\begin{array}{l}\mathrm{UO}_{2} ; 26 \% \text { enriched U in central pin } \\
\text { (except } 3.8 \mathrm{~cm} \text { of fuel at midplane } \\
93 \% \text { enriched), 20\% enriched in } \\
\text { outer pins; } \\
\text { O/M }=2.00\end{array}$ \\
\hline Geometry & Stack of dished pellets \\
\hline Fissile height & $34.3 \mathrm{~cm}$ \\
\hline $\begin{array}{l}\text { Smear } \\
\text { density }\end{array}$ & $9.2 \mathrm{~g} / \mathrm{cm}^{3}$ \\
\hline
\end{tabular}

\begin{tabular}{|l|l|}
\hline \multicolumn{2}{|c|}{ CLADDING } \\
\hline Material & 316 stainless steel \\
\hline OD & $5.84 \mathrm{~mm}$ \\
\hline Thickness & $0.38 \mathrm{~mm}$ \\
\hline
\end{tabular}

\begin{tabular}{|l|l|}
\hline \multicolumn{2}{|l|}{ PRE-IRRADIATION CHARACTERISTICS } \\
\hline BU (peak axial) & None \\
\hline Peak linear power & (not applicable) \\
\hline $\begin{array}{l}\text { Peak internal clad } \\
\text { temperature }\end{array}$ & (not applicable) \\
\hline $\begin{array}{l}\text { Fluence } \\
\text { (spectrum) }\end{array}$ & (not applicable) \\
\hline
\end{tabular}

\section{OTHER FUEL SAMPLE INFORMATION}

All pins were wire-wrapped.

\begin{tabular}{|l|l|}
\hline \multicolumn{2}{|c|}{ TEST CONDITIONS, MEASUREMENTS, \& ANALYSES } \\
\hline $\begin{array}{l}\text { Coolant } \\
\text { environment }\end{array}$ & $\begin{array}{l}\text { Sodium flow at } 870 \mathrm{~cm}^{3} / \mathrm{s} \text {; inlet temperature } \\
\text { increasing from } 425^{\circ} \mathrm{C} \text { to } 555^{\circ} \mathrm{C} \text { during the } \\
\text { transient }\end{array}$ \\
\hline $\begin{array}{l}\text { Transient } \\
\text { conditions }\end{array}$ & $\begin{array}{l}\text { Constant power of } ~ 70 \mathrm{~kW} / \mathrm{m}(\sim 140 \mathrm{~kW} / \mathrm{m} \text { in the } \\
\text { fully-enriched section) in the central pin for } ~ \\
\text { The power generated in the outer pins was 10- } \\
\text { 20\%less than in the center pin. }\end{array}$ \\
\hline $\begin{array}{l}\text { Fuel sample } \\
\text { configuration } \\
\text { in the test }\end{array}$ & $\begin{array}{l}\text { Seven-pin bundle, with center pin higher } \\
\text { enriched than edge pins }\end{array}$ \\
\hline $\begin{array}{l}\text { Test vehicle \& } \\
\text { experiment } \\
\text { system }\end{array}$ & $\begin{array}{l}\text { Mark-IIA integral sodium loop; 7-pin test train } \\
\text { with flowing sodium area }=1.86 \mathrm{~cm} \text { ' surrounded } \\
\text { by a 0.9-mm-thick stainless steel wall }\end{array}$ \\
\hline $\begin{array}{l}\text { Measurements } \\
\text { /diagnostics } \\
\text { during test }\end{array}$ & $\begin{array}{l}\text { Coolant pressure, flow rate, and temperature; } \\
\text { structure temperatures. }\end{array}$ \\
\hline $\begin{array}{l}\text { Posttest } \\
\text { coolant flow } \\
\text { condition }\end{array}$ & No flow blockage \\
\hline $\begin{array}{l}\text { Posttest } \\
\text { condition of } \\
\text { test sample(s) }\end{array}$ & All pins remained intact. \\
\hline $\begin{array}{l}\text { Posttest } \\
\text { measurements } \\
\text { \& information }\end{array}$ & $\begin{array}{l}\text { Neutron radiography; macro- and micro- } \\
\text { examinations; eddy-current examination of the } \\
\text { cladding; gamma scanning; X-radiography; gas } \\
\text { analysis; alpha-radiography }\end{array}$ \\
\hline $\begin{array}{l}\text { Thalyses } \\
\text { Thermal-hydraulic and cladding mechanics }\end{array}$ \\
\hline
\end{tabular}

\section{KEY SUMMARY INFORMATION ABOUT THE TEST}

Test D2 was a higher-power version of test D1 to cause pin fail ure and obtain information on the conditions required to achieve the release of a small amount of molten fuel from a failed fresh $\mathrm{UO}_{2}$ pin into flowing sodium and, if failure occurred, to provide information useful in determining the mechanism and cause of the release, the extent of any molten-fuel/coolant interaction, the nature of any flowimpediment caused by the fuel release, and whether any damage to adjacent pins tends to propagate additional pin failure. The test caused more fuel to melt than in D1, but pin failure did not occur in D2 either. The outlet sodium temperature at the end of the power transient was 690 ${ }^{\circ} \mathrm{C}$. Posttest visual examination revealed no anomalies on the pin exteriors. There was no statistically-significant change in length or bow of any of the pins before their wire wraps were removed, and there was little or no change in cladding diameter. Destructive examination showed that up to $75 \%$ of the area of the fully-enriched section of the central pin had melted, and small amounts of molten fuel flowed through fissures in the solid fuel and contacted the cladding, where the fuel was immediately quenched. Along the $26 \%$-enriched regions of the central pin, the maximum amount of melting was $40 \%$. In that pin, $\sim 6 \mathrm{~cm}$ length of central void immediately below the fully-enriched pellets was filled with fully-enriched fuel, and the central void immediately above the fully-enriched pellets had a layer of fully-enriched fuel. In many axial locations, melted fuel had penetrated to the cladding through pellet cracks or spaces between pellets, but the fuelcladding contact had practically no effect on the cladding. Molten fuel significantly flowed downward into available central void spaces of pellets below. Central voids had formed in all of the fuel pins. 


\section{Appendix R: One-page Summaries of S-series Tests}

One-page summaries of each of the S-series tests are included here. These summaries can also be found in TREXR [6]. 


\section{Summary of TREAT Test S-2 \\ Performed 11 September 1968}

Transient No. unknown

\section{PRE-TEST SAMPLE CHARACTERIZATION}

\begin{tabular}{|l|l|}
\hline \multicolumn{2}{|c|}{ FUEL } \\
\hline Form & $\mathrm{He}$-bonded $\mathrm{UO}_{2}$ \\
\hline Composition & $\mathrm{UO}_{2}-10 \%$ enriched U \\
\hline Geometry & Solid pellet \\
\hline Fissile height & $14.9 \mathrm{~cm}$ \\
\hline $\begin{array}{l}\text { Smear } \\
\text { density }\end{array}$ & $84-90 \%$ of theoretical density \\
\hline
\end{tabular}

\begin{tabular}{|l|l|}
\hline \multicolumn{2}{|c|}{ CLADDING } \\
\hline Material & Type 304 stainless steel \\
\hline OD & $7.37 \mathrm{~mm}$ \\
\hline Thickness & $0.38 \mathrm{~mm}$ \\
\hline
\end{tabular}

\begin{tabular}{|l|l|}
\hline \multicolumn{2}{|l|}{ PRE-IRRADIATION CHARACTERISTICS } \\
\hline BU (peak axial) & Zero \\
\hline Peak linear power & (not applicable) \\
\hline $\begin{array}{l}\text { Peak internal clad } \\
\text { temperature }\end{array}$ & (not applicable) \\
\hline $\begin{array}{l}\text { Fluence } \\
\text { (spectrum) }\end{array}$ & (zero) \\
\hline
\end{tabular}

\section{OTHER FUEL SAMPLE INFORMATION}

Fueled section in each pin was a stack of pellet, with an unenriched pellet at each end. Total of $41 \mathrm{~g}$ of fuel in each fueled pin ( $205 \mathrm{~g}$ total in the test). Top and bottom enriched pellets received $14 \%$ more energy than the other enriched pellets. There was $200 \mathrm{~g}$ of sodium in the test. The fuel in the pins was helium bonded to the cladding at normal temperature and pressure. A $15.5 \mathrm{~cm}$ long, unfueled section, filled with helium at $0.1 \mathrm{MPa}$, was located in the cladding above the fuel but sealed off from the fueled section. Ratio of sodium/fueled pin cross-sectional areas $=2.35$.

\begin{tabular}{|c|c|}
\hline \multicolumn{2}{|c|}{ TEST CONDITIONS, MEASUREMENTS, \& ANALYSES } \\
\hline $\begin{array}{l}\text { Coolant } \\
\text { environment }\end{array}$ & $\begin{array}{l}\text { Stagnant sodium at } 190^{\circ} \mathrm{C} \text { before the power } \\
\text { burst. }\end{array}$ \\
\hline $\begin{array}{l}\text { Transient } \\
\text { conditions }\end{array}$ & $\begin{array}{l}\text { Three power-burst transients, with coddowns in } \\
\text { between, generating average fission energies of } \\
900,1380 \text {, and } 1420 \mathrm{~J} / \mathrm{g} \mathrm{UO} \\
\text {, respectively. The } \\
\text { full-width-at-half-maximum of the last transient } \\
\text { was about } 220 \mathrm{~ms} \text {. }\end{array}$ \\
\hline $\begin{array}{l}\text { Fuel sample } \\
\text { configuration } \\
\text { in the test }\end{array}$ & $\begin{array}{l}\text { Square }(3 \times 3) \text { array of nine pins: } 5 \text { fuel rods } \\
\text { and } 4 \text { hollow dummy pins. } \\
\text { The dummy pins contained helium at } 1 \text { bar } \\
\text { pressure inside Type- } 304 \text { stainless-steel tubing } \\
30.5 \mathrm{~cm} \text { long, } 0.737 \mathrm{~cm} \text { OD and } 0.305 \text { mm wall.) }\end{array}$ \\
\hline $\begin{array}{l}\text { Test vehicle \& } \\
\text { experiment } \\
\text { system }\end{array}$ & $\begin{array}{l}\text { Piston autoclave with pin bundle submerged in } \\
\text { sodium located inside a Zircaloy sleeve. }\end{array}$ \\
\hline $\begin{array}{l}\text { Measurements } \\
\text { /diagnostics } \\
\text { during test }\end{array}$ & $\begin{array}{l}\text { Temperatures were measured by four tungsten- } \\
\text { rhenium TCs. (The TCs had time constant = } 15 \\
\text { ms.) Pressure transducer measured autoclave } \\
\text { pressure. Upward motion of a } 260 \mathrm{~g} \text { piston } \\
\text { located immediately above the fuel-pin bunde } \\
\text { was measured by a linear motion transducer. }\end{array}$ \\
\hline $\begin{array}{l}\text { Posttest } \\
\text { coolant flow } \\
\text { condition }\end{array}$ & (Not relevant.) \\
\hline $\begin{array}{l}\text { Posttest } \\
\text { condition of } \\
\text { test sample(s) }\end{array}$ & $\begin{array}{l}\text { Central pin had failed with about } 60 \% \text { of the fuel } \\
\text { having left the cladding. The cladding had split, } \\
\text { but there was no sign of melting or cladding } \\
\text { expansion at that location. The other pins } \\
\text { remained intact. }\end{array}$ \\
\hline $\begin{array}{l}\text { Posttest } \\
\text { measurements } \\
\text { \& information }\end{array}$ & $\begin{array}{l}\text { Neutron radiography of the fuel sample after } \\
\text { each transient. }\end{array}$ \\
\hline $\begin{array}{l}\text { Posttest } \\
\text { analyses }\end{array}$ & Thermal-mechanical and heat transfer analyses. \\
\hline
\end{tabular}

\section{KEY SUMMARY INFORMATION ABOUT THE TEST}

Test S-2, in conjunction with tests S-3 and S-4, investigated the effect of increasing fuel energy on the consequences of fuel pin failure. In Test S-2, the pins were subjected to three power burst transients of increasingly-greater energy. The cladding of the central fuel pin cladding failed at $980 \mathrm{~kJ} / \mathrm{g}$ during the third transient by a tear in the cladding, causing an initial $0.55 \mathrm{MPa}$ pressure pulse, followed by a $1.2 \mathrm{MPa}$ pulse (the highest pressure recorded during the test) when the pin energy reached $1170 \mathrm{~kJ} / \mathrm{g}$ (the latter probably after fuelsodium contact). About $59 \%$ of the fuel in the central pin escaped from the cladding. Diametral measurements showed no dimensional changes in the vicinity of the tear, but they showed about $3 \%$ increase in diameter at the fuel midplane. The other pins remained intact during the test and showed no dimensional changes. No signs of cladding melting were found. 


\section{Summary of TREAT Test S-3}

Performed 1 November 1968

Transient No. unknown

\section{PRE-TEST SAMPLE CHARACTERIZATION}

\begin{tabular}{|l|l|}
\hline \multicolumn{2}{|c|}{ FUEL } \\
\hline Form & He-bonded $\mathrm{UO}_{2}$ \\
\hline Composition & $\mathrm{UO}_{2}-10 \%$ enriched U \\
\hline Geometry & Solid pellet \\
\hline Fissile height & $14.9 \mathrm{~cm}$ \\
\hline $\begin{array}{l}\text { Smear } \\
\text { density }\end{array}$ & $84-90 \%$ of theoretical density \\
\hline
\end{tabular}

\begin{tabular}{|l|l|}
\hline \multicolumn{2}{|c|}{ CLADDING } \\
\hline Material & Type 304 stainless steel \\
\hline OD & $7.37 \mathrm{~mm}$ \\
\hline Thickness & $0.38 \mathrm{~mm}$ \\
\hline
\end{tabular}

\begin{tabular}{|l|l|}
\hline \multicolumn{2}{|l|}{ PRE-IRRADIATION CHARACTERISTICS } \\
\hline BU (peak axial) & Zero \\
\hline Peak linear power & (not applicable) \\
\hline $\begin{array}{l}\text { Peak internal clad } \\
\text { temperature }\end{array}$ & (not applicable) \\
\hline $\begin{array}{l}\text { Fluence } \\
\text { (spectrum) }\end{array}$ & (zero) \\
\hline
\end{tabular}

\section{OTHER FUEL SAMPLE INFORMATION}

Fueled section in each pin was a stack of pellet, with an unenriched pellet at each end. Total of $41 \mathrm{~g}$ of fuel in each fueled pin ( $205 \mathrm{~g}$ total in the test). Top and bottom enriched pellets received $14 \%$ more energy than the other enriched pellets. There was $200 \mathrm{~g}$ of sodium in the test. The fuel was helium bonded to the cladding at normal temperature and pressure. A $15.5 \mathrm{~cm}$ long, unfueled section, filled with helium at $0.1 \mathrm{MPa}$, was located in the cladding above the fuel but sealed off from the fueled section. Ratio of sodium/fueled pin cross-sectional areas $=2.35$.

\begin{tabular}{|c|c|}
\hline \multicolumn{2}{|c|}{ TEST CONDITIONS, MEASUREMENTS, \& ANALYSES } \\
\hline $\begin{array}{l}\text { Coolant } \\
\text { environment }\end{array}$ & $\begin{array}{l}\text { Stagnant sodium at } 165^{\circ} \mathrm{C} \text { before the power } \\
\text { burst. }\end{array}$ \\
\hline $\begin{array}{l}\text { Transient } \\
\text { conditions }\end{array}$ & $\begin{array}{l}\text { Two power-burst transients, generating average } \\
\text { fission energies of } 0.99 \mathrm{~kJ} / \mathrm{g} \text { and } 1.92 \mathrm{~kJ} / \mathrm{g} \text {, } \\
\text { respectively, in the test fuel. The full-width-at- } \\
\text { half-maximum of the last transient was about } \\
220 \mathrm{~ms} \text {. }\end{array}$ \\
\hline $\begin{array}{l}\text { Fuel sample } \\
\text { configuration } \\
\text { in the test }\end{array}$ & $\begin{array}{l}\text { Square }(3 \times 3) \text { open array of nine pins: } 5 \text { fuel } \\
\text { rods and } 4 \text { hollow dummy pins. } \\
\text { The dummy pins contained helium at } 1 \text { bar } \\
\text { pressure inside Type- } 304 \text { stainless-steel tubing } \\
30.5 \mathrm{~cm} \text { long, } 0.737 \mathrm{~cm} \text { OD and } 0.305 \mathrm{~mm} \text { wall.) }\end{array}$ \\
\hline $\begin{array}{l}\text { Test vehicle \& } \\
\text { experiment } \\
\text { system }\end{array}$ & $\begin{array}{l}\text { Piston autoclave with pin bundle submerged in } \\
\text { sodium located inside a Zircaloy sleeve. }\end{array}$ \\
\hline $\begin{array}{l}\text { Measurements } \\
\text { /diagnostics } \\
\text { during test }\end{array}$ & $\begin{array}{l}\text { Temperatures were measured by four tungsten- } \\
\text { rhenium TCs. (The TCs had time constant }=15 \\
\text { ms.) Pressure transducer measured autoclave } \\
\text { pressure. Upward motion of a } 260 \mathrm{~g} \text { piston } \\
\text { located immediately above the fuel-pin bunde } \\
\text { was measured by a linear motion transducer. }\end{array}$ \\
\hline $\begin{array}{l}\text { Posttest } \\
\text { coolant flow } \\
\text { condition }\end{array}$ & (Not relevant.) \\
\hline $\begin{array}{l}\text { Posttest } \\
\text { condition of } \\
\text { test sample(s) }\end{array}$ & $\begin{array}{l}\text { All five fueled pins were completely disrupted } \\
\text { and melted throughout the fuel region. Much of } \\
\text { the fuel was in the form of hollow cylinders. All } \\
\text { dummy pins were also breached. }\end{array}$ \\
\hline $\begin{array}{l}\text { Posttest } \\
\text { measurements } \\
\text { \& information }\end{array}$ & $\begin{array}{l}\text { Neutron radiography of the fuel sample after } \\
\text { each transient. }\end{array}$ \\
\hline $\begin{array}{l}\text { Posttest } \\
\text { analyses }\end{array}$ & Thermal-mechanical and heat transfer analyses. \\
\hline
\end{tabular}

\section{KEY SUMMARY INFORMATION ABOUT THE TEST}

Test S-3, in conjunction with tests S-2 and S-4, investigated the effect of increasing fuel energy on the consequences of fuel pin failure. In test S-3, there were five separate pressure pulses: two ( 3.1 and $2.8 \mathrm{MPa}$ ) occurring at $1.86 \mathrm{~kJ} / \mathrm{g}$ fuel energy, and the third pulse (largest observed in the test, having $3.7 \mathrm{MPa}$ and $2.7 \mathrm{~ms}$ width) occurring about $10 \mathrm{~ms}$ later. The acceleration of the piston occurred simultaneously with the generation of the pressure pulses. A peak sodium temperature of $1100^{\circ} \mathrm{C}$ was recorded by a thermocouple located in the upper part of the fueled section of the fuel pin about $0.8 \mathrm{~s}$ after peak power.

Only small ( 1-cm long) hollow, cylindrical sections ( $4.8 \mathrm{~mm} \mathrm{ID,6.7} \mathrm{mm} \mathrm{OD)} \mathrm{of} \mathrm{fused} \mathrm{UO2} \mathrm{remained} \mathrm{after} \mathrm{the} \mathrm{test.} \mathrm{The} \mathrm{cylindrical}$ sections were very friable and readily broke into fine particles upon application of slight force. The lower portions of the dummy pins failed by melting. The mass-mean fuel particulate diameter was $2.7 \mathrm{~mm}$. The calculated energy conversion was $0.002 \%$. 


\section{Summary of TREAT Test S-4 \\ Performed 19 August 1969 \\ Transient No. unknown}

\section{PRE-TEST SAMPLE CHARACTERIZATION}

\begin{tabular}{|l|l|}
\hline \multicolumn{2}{|c|}{ FUEL } \\
\hline Form & He-bonded $\mathrm{UO}_{2}$ \\
\hline Composition & $\mathrm{UO}_{2}-10 \%$ enriched U \\
\hline Geometry & Solid pellet \\
\hline Fissile height & $14.9 \mathrm{~cm}$ \\
\hline $\begin{array}{l}\text { Smear } \\
\text { density }\end{array}$ & $84-90 \%$ of theoretical density \\
\hline
\end{tabular}

\begin{tabular}{|l|l|}
\hline \multicolumn{2}{|c|}{ CLADDING } \\
\hline Material & Type 304 stainless steel \\
\hline OD & $7.37 \mathrm{~mm}$ \\
\hline Thickness & $0.38 \mathrm{~mm}$ \\
\hline
\end{tabular}

\begin{tabular}{|l|l|}
\hline \multicolumn{2}{|l|}{ PRE-IRRADIATION CHARACTERISTICS } \\
\hline BU (peak axial) & Zero \\
\hline Peak linear power & (not applicable) \\
\hline $\begin{array}{l}\text { Peak internal clad } \\
\text { temperature }\end{array}$ & (not applicable) \\
\hline $\begin{array}{l}\text { Fluence } \\
\text { (spectrum) }\end{array}$ & (zero) \\
\hline
\end{tabular}

\section{OTHER FUEL SAMPLE INFORMATION}

Fueled section in each pin was a stack of pellet, with an unenriched pellet at each end. Total of $41 \mathrm{~g}$ of fuel in each fueled pin ( $205 \mathrm{~g}$ total in the test). Top and bottom enriched pellets received $14 \%$ more energy than the other enriched pellets. There was $200 \mathrm{~g}$ of sodium in the test. The fuel was helium bonded to the cladding at normal temperature and pressure. A $15.5 \mathrm{~cm}$ long, unfueled section, filled with helium at $0.1 \mathrm{MPa}$, was located in the cladding above the fuel but sealed off from the fueled section. Ratio of sodium/fueled pin cross-sectional areas $=2.35$.

\begin{tabular}{|c|c|}
\hline \multicolumn{2}{|c|}{ TEST CONDITIONS, MEASUREMENTS, \& ANALYSES } \\
\hline $\begin{array}{l}\text { Coolant } \\
\text { environment }\end{array}$ & $\begin{array}{l}\text { Stagnant sodium at } 165^{\circ} \mathrm{C} \text { before the power } \\
\text { burst. }\end{array}$ \\
\hline $\begin{array}{l}\text { Transient } \\
\text { conditions }\end{array}$ & $\begin{array}{l}\text { One power burst transient with } 160 \mathrm{~ms} \text { full-width- } \\
\text { at-half-maximum generating } 3.0 \mathrm{~kJ} / \mathrm{g} \text { in the test } \\
\text { fuel. }\end{array}$ \\
\hline $\begin{array}{l}\text { Fuel sample } \\
\text { configuration } \\
\text { in the test }\end{array}$ & $\begin{array}{l}\text { Square ( } 3 \times 3 \text { ) open array of nine pins: } 5 \text { fuel } \\
\text { rods and } 4 \text { hollow dummy pins. } \\
\text { The dummy pins contained helium at } 1 \text { bar } \\
\text { pressure inside Type- } 304 \text { stainless-steel tubing } \\
30.5 \mathrm{~cm} \text { long, } 0.737 \mathrm{~cm} \text { OD and } 0.305 \mathrm{~mm} \text { wall.) }\end{array}$ \\
\hline $\begin{array}{l}\text { Test vehicle \& } \\
\text { experiment } \\
\text { system }\end{array}$ & $\begin{array}{l}\text { Piston autoclave with pin bundle submerged in } \\
\text { sodium located inside a Zircaloy sleeve. }\end{array}$ \\
\hline $\begin{array}{l}\text { Measurements } \\
\text { /diagnostics } \\
\text { during test }\end{array}$ & $\begin{array}{l}\text { Temperatures were measured by four tungsten- } \\
\text { rhenium TCs. (The TCs had time constant }=15 \\
\text { ms.) Pressure transducer measured autoclave } \\
\text { pressure. Upward motion of a } 260 \mathrm{~g} \text { piston } \\
\text { located immediately above the fuel-pin bunde } \\
\text { was measured by a linear motion transducer. }\end{array}$ \\
\hline $\begin{array}{l}\text { Posttest } \\
\text { coolant flow } \\
\text { condition }\end{array}$ & (Not relevant.) \\
\hline $\begin{array}{l}\text { Posttest } \\
\text { condition of } \\
\text { test sample(s) }\end{array}$ & $\begin{array}{l}\text { Gross destruction of all five fuel pins and the four } \\
\text { dummy pins. }\end{array}$ \\
\hline $\begin{array}{l}\text { Posttest } \\
\text { measurements } \\
\& \text { information }\end{array}$ & $\begin{array}{l}\text { Neutron radiography of the fuel sample after } \\
\text { each transient. }\end{array}$ \\
\hline $\begin{array}{l}\text { Posttest } \\
\text { analyses }\end{array}$ & Thermal-mechanical and heat transfer analyses. \\
\hline
\end{tabular}

\section{KEY SUMMARY INFORMATION ABOUT THE TEST}

Test S-4, in conjunction with tests S-2 and S-3, investigated the effect of increasing fuel energy on the consequences of fuel pin failure. In Test S-4 there were five distinct pressure pulses. The first two occurred $30 \mathrm{~ms}$ after peak power, at the time $2.0 \mathrm{~kJ} / \mathrm{g}$ had been generated in the test fuel, At about $34 \mathrm{~ms}$ after peak power, a pressure pulse with two peaks occurred, one of $7 \mathrm{MPa}$ and the other of about $12 \mathrm{MPa}$. At about $42 \mathrm{~ms}$ after peak power, a $9 \mathrm{MPa}$ peak pressure pulse occurred. The acceleration of the piston occurred simultaneously with the generation of the pressure pulses. Hot fuel and fuel-pin debris were propelled upward during the test. Cladding melting was much more extensive than in test S-3.

The mass-mean diameter of the fuel residue was about $1.2 \mathrm{~mm}$. The calculated conversion from nuclear energy to mechanical energy was estimated to be $0.025 \%$, ten times higher than in test S-3. 


\section{Summary of TREAT Test S-5 \\ Performed 3 June 1970}

Transient No. unknown

\section{PRE-TEST SAMPLE CHARACTERIZATION}

\begin{tabular}{|l|l|}
\hline \multicolumn{2}{|c|}{ FUEL } \\
\hline Form & He-bonded $\mathrm{UO}_{2}$ \\
\hline Composition & $\mathrm{UO}_{2}-10 \%$ enriched U \\
\hline Geometry & Solid pellet \\
\hline Fissile height & $14.9 \mathrm{~cm}$ \\
\hline $\begin{array}{l}\text { Smear } \\
\text { density }\end{array}$ & $84-90 \%$ of theoretical density \\
\hline
\end{tabular}

\begin{tabular}{|l|l|}
\hline \multicolumn{2}{|c|}{ CLADDING } \\
\hline Material & Type 304 stainless steel \\
\hline OD & $7.37 \mathrm{~mm}$ \\
\hline Thickness & $0.38 \mathrm{~mm}$ \\
\hline
\end{tabular}

\begin{tabular}{|l|l|}
\hline \multicolumn{2}{|l|}{ PRE-IRRADIATION CHARACTERISTICS } \\
\hline BU (peak axial) & Zero \\
\hline Peak linear power & (not applicable) \\
\hline $\begin{array}{l}\text { Peak internal clad } \\
\text { temperature }\end{array}$ & (not applicable) \\
\hline $\begin{array}{l}\text { Fluence } \\
\text { (spectrum) }\end{array}$ & (zero) \\
\hline
\end{tabular}

\section{OTHER FUEL SAMPLE INFORMATION}

Fueled section in each pin was a stack of pellet, with an unenriched pellet at each end. Total of $41 \mathrm{~g}$ of fuel in each fueled pin ( $205 \mathrm{~g}$ total fuel in the test). Top and bottom enriched pellets received $14 \%$ more energy than the other enriched pellets. There was $200 \mathrm{~g}$ of sodium in the test. The fuel pins (including upper blanket sections) and dummy pins had been sealed under vacuum of $5 x$ $10^{5}$ torr. A $15.5 \mathrm{~cm}$ long, unfueled section, filled with helium at $0.1 \mathrm{MPa}$, was located in the cladding above the fuel but sealed off from the fueled section. Ratio of sodium/fueled pin cross-sectional areas $=2.35$.

\begin{tabular}{|c|c|}
\hline \multicolumn{2}{|c|}{ TEST CONDITIONS, MEASUREMENTS, \& ANALYSES } \\
\hline $\begin{array}{l}\text { Coolant } \\
\text { environment }\end{array}$ & $\begin{array}{l}\text { Stagnant sodium at } 170^{\circ} \mathrm{C} \text { before the power } \\
\text { burst. }\end{array}$ \\
\hline $\begin{array}{l}\text { Transient } \\
\text { conditions }\end{array}$ & $\begin{array}{l}\text { The full-width-at-half-maximum of the last } \\
\text { transient was about } 195 \mathrm{~ms} \text {. The transient } \\
\text { generated an average of } 2.13 \mathrm{~kJ} / \mathrm{g} \text { within the fue } \\
\text { mass in the test. }\end{array}$ \\
\hline $\begin{array}{l}\text { Fuel sample } \\
\text { configuration } \\
\text { in the test }\end{array}$ & $\begin{array}{l}\text { Square }(3 \times 3) \text { open array of nine pins: } 5 \text { fuel } \\
\text { rods and } 4 \text { hollow dummy pins. } \\
\text { The dummy pins contained helium at } 1 \text { bar } \\
\text { pressure inside Type- } 304 \text { stainless-steel tubing } \\
30.5 \mathrm{~cm} \text { long, } 0.737 \mathrm{~cm} \text { OD and } 0.305 \text { mm wall.) }\end{array}$ \\
\hline $\begin{array}{l}\text { Test vehicle \& } \\
\text { experiment } \\
\text { system }\end{array}$ & $\begin{array}{l}\text { Piston autoclave with pin bundle submerged in } \\
\text { sodium located inside a Zircaloy sleeve. }\end{array}$ \\
\hline $\begin{array}{l}\text { Measurements } \\
\text { /diagnostics } \\
\text { during test }\end{array}$ & $\begin{array}{l}\text { Temperatures were measured by four tungsten- } \\
\text { rhenium TCs. (The TCs had time constant = } 15 \\
\text { ms.) Pressure transducer measured autoclave } \\
\text { pressure. Upward motion of a } 260 \mathrm{~g} \text { piston } \\
\text { located immediately above the fuel-pin bunde } \\
\text { was measured by a linear motion transducer. }\end{array}$ \\
\hline $\begin{array}{l}\text { Posttest } \\
\text { coolant flow } \\
\text { condition }\end{array}$ & (Not relevant.) \\
\hline $\begin{array}{l}\text { Posttest } \\
\text { condition of } \\
\text { test sample(s) }\end{array}$ & $\begin{array}{l}\text { Gross disruption of the fuel pins, with the fuel } \\
\text { residue being highly fragmented. }\end{array}$ \\
\hline $\begin{array}{l}\text { Posttest } \\
\text { measurements } \\
\text { \& information }\end{array}$ & $\begin{array}{l}\text { Neutron radiography of the fuel sample after } \\
\text { each transient. }\end{array}$ \\
\hline $\begin{array}{l}\text { Posttest } \\
\text { analyses }\end{array}$ & Thermal-mechanical and heat transfer analyses. \\
\hline
\end{tabular}

\section{KEY SUMMARY INFORMATION ABOUT THE TEST}

Test S- 5 had the objective of investigating whether large pressures and work energies would be produced in the absence of bond gas within the pins. (Release of a gas bond from failed cladding could blanket the fuel and reduce fuel-sodium contact.) However, because the fuel pellets were $93 \%$ (not 100\%) of theoretical density, some gas within the fuel pellet could have had an effect. The initial pressure pulse $(0.5 \mathrm{MPa})$ occurred when the fuel energy generated had reached $1.80 \mathrm{~kJ} / \mathrm{g}$, simultaneous with the acceleration of the piston. Sixteen pressure pulses higher than $0.3 \mathrm{MPa}$ were recorded, the highest being $20 \mathrm{MPa}$. There was more fuel damage than in Test S-3, and more fuel fragmentation and particulate. The mass-mean residue diameter was about $0.2 \mathrm{~mm}$. A conservative maximum calculated nuclear to mechanical energy conversion during the test was estimated to be $0.04-0.2 \%$ depending upon assumptions. 


\section{Summary of TREAT Test S-6 \\ Performed 24 Nov 1970}

Transient No. 1348

\section{PRE-TEST SAMPLE CHARACTERIZATION}

\begin{tabular}{|l|l|}
\hline \multicolumn{2}{|c|}{ FUEL } \\
\hline Form & He-bonded $\mathrm{UO}_{2}$ \\
\hline Composition & $\begin{array}{l}\mathrm{UO}_{2}-\text { uranium enrichment 13\% } \\
\text { (max.) in central pin, 10\% (max.) in } \\
\text { edge pins }\end{array}$ \\
\hline Geometry & Solid pellet \\
\hline Fissile height & $12.4 \mathrm{~cm}$ \\
\hline $\begin{array}{l}\text { Smear } \\
\text { density }\end{array}$ & $84-90 \%$ of theoretical density \\
\hline
\end{tabular}

\begin{tabular}{|l|l|}
\hline \multicolumn{2}{|c|}{ CLADDING } \\
\hline Material & Type 304 stainless steel \\
\hline OD & $7.37 \mathrm{~mm}$ \\
\hline Thickness & $0.38 \mathrm{~mm}$ \\
\hline
\end{tabular}

PRE-IRRADIATION CHARACTERISTICS

\begin{tabular}{|l|l|}
\hline BU (peak axial) & Zero \\
\hline Peak linear power & (not applicable) \\
\hline $\begin{array}{l}\text { Peak internal clad } \\
\text { temperature }\end{array}$ & (not applicable) \\
\hline $\begin{array}{l}\text { Fluence } \\
\text { (spectrum) }\end{array}$ & (zero) \\
\hline
\end{tabular}

\section{OTHER FUEL SAMPLE INFORMATION}

Fueled section in each pin was a stack of pellets, with an unenriched pellet at each end. Total of $286 \mathrm{~g}$ of fuel in the test. Pins were wrapped with $1.6 \mathrm{~mm}$ diameter wire. Central pin received $9 \%$ more power than each edge pin. There was $200 \mathrm{~g}$ of sodium in the test. Ratio of sodium/fueled pin cross-sectional areas $=0.94$. A $15.5 \mathrm{~cm}$ long, unfueled section, filled with helium at 0.1 $\mathrm{MPa}$, was located in the cladding above the fuel but sealed off from the fueled section.

\begin{tabular}{|c|c|}
\hline \multicolumn{2}{|c|}{ TEST CONDITIONS, MEASUREMENTS, \& ANALYSES } \\
\hline $\begin{array}{l}\text { Coolant } \\
\text { environment }\end{array}$ & $\begin{array}{l}\text { Stagnant sodium at } 210^{\circ} \mathrm{C} \text { before the power } \\
\text { burst. }\end{array}$ \\
\hline $\begin{array}{l}\text { Transient } \\
\text { conditions }\end{array}$ & $\begin{array}{l}\text { Power burst with } 170 \text { ms full-width-at-half- } \\
\text { maximum, } 2.06 \mathrm{~kJ} / \mathrm{g} \text { in the fuel }\end{array}$ \\
\hline $\begin{array}{l}\text { Fuel sample } \\
\text { configuration } \\
\text { in the test }\end{array}$ & Bunde of 7 wire-wrapped fuel pins. \\
\hline $\begin{array}{l}\text { Test vehicle \& } \\
\text { experiment } \\
\text { system }\end{array}$ & $\begin{array}{l}\text { Piston autoclave with pin bundle submerged in } \\
\text { sodium located inside a Zircal oy sleeve. }\end{array}$ \\
\hline $\begin{array}{l}\text { Measurements } \\
\text { /diagnostics } \\
\text { during test }\end{array}$ & $\begin{array}{l}\text { Temperatures were measured by four tungsten- } \\
\text { rhenium TCs. (The TCs had time constant }=15 \\
\text { ms.) Pressure transducer measured autoclave } \\
\text { pressure. Upward motion of a } 260 \mathrm{~g} \text { piston } \\
\text { located immediately above the fuel-pin bundle } \\
\text { was measured by a linear motion transducer. } \\
\text { The TREAT Fast Neutron Hodoscope was used } \\
\text { to measure fuel motion. }\end{array}$ \\
\hline $\begin{array}{l}\text { Posttest } \\
\text { coolant flow } \\
\text { condition }\end{array}$ & (Not relevant.) \\
\hline $\begin{array}{l}\text { Posttest } \\
\text { condition of } \\
\text { test sample(s) }\end{array}$ & $\begin{array}{l}\text { Central pin had failed, and about } 60 \% \text { of its fuel } \\
\text { had left the cladding. The cladding had split, but } \\
\text { there was no sign of melting or cladding } \\
\text { expansion at that location. The other pins } \\
\text { remained intact. }\end{array}$ \\
\hline $\begin{array}{l}\text { Posttest } \\
\text { measurements } \\
\& \text { information }\end{array}$ & $\begin{array}{l}\text { Neutron radiography of the fuel sample after } \\
\text { each transient. }\end{array}$ \\
\hline $\begin{array}{l}\text { Posttest } \\
\text { analyses }\end{array}$ & Thermal-mechanical and heat transfer analyses. \\
\hline
\end{tabular}

\section{KEY SUMMARY INFORMATION ABOUT THE TEST}

Test S-6 investigated the effect of a more-closely-packed fuel-pin geometry on fuel-codant interaction effects, compared to the earlier tests S-2 through S-5. In S-6, cladding failure occurred when the fuel energy had reached $1.59 \mathrm{~kJ} / \mathrm{g}$. The largest pressure pulse reached $14 \mathrm{MPa}$. Based on the average fuel energy at initial cladding failure, the calculated energy conversion ratio was $0.15 \%$ to $0.037 \%$ to $0.2 \%$ depending on whether damped pressure oscillations are included in the calculation. The fuel debris particle sizes were overall much like those in tests S-3 and S-4, but they were not as small as the sizes in test S-5 which used evacuated pins. 


\section{Summary of TREAT Test S-7 \\ Performed 21 May 1971 \\ Transient No. 1376}

\section{PRE-TEST SAMPLE CHARACTERIZATION}

\begin{tabular}{|l|l|}
\hline \multicolumn{2}{|c|}{ FUEL } \\
\hline Form & He-bonded $\mathrm{UO}_{2}$ \\
\hline Composition & $\begin{array}{l}\mathrm{UO}_{2}-\text { uranium enrichment 13\% in } \\
\text { central pin, 10\% in edge pins }\end{array}$ \\
\hline Geometry & Solid pellet \\
\hline Fissile height & $\sim 13 \mathrm{~cm}$ \\
\hline $\begin{array}{l}\text { Smear } \\
\text { density }\end{array}$ & $85 \%$ of theoretical \\
\hline
\end{tabular}

\begin{tabular}{|l|l|}
\hline \multicolumn{2}{|c|}{ CLADDING } \\
\hline Material & Type 316 stainless steel \\
\hline OD & $5.84 \mathrm{~mm}$ \\
\hline Thickness & $0.38 \mathrm{~mm}$ \\
\hline
\end{tabular}

\begin{tabular}{|l|l|}
\hline \multicolumn{2}{|l|}{ PRE-IRRADIATION CHARACTERISTICS } \\
\hline BU (peak axial) & Zero \\
\hline Peak linear power & (not applicable) \\
\hline $\begin{array}{l}\text { Peak internal clad } \\
\text { temperature }\end{array}$ & (not applicable) \\
\hline $\begin{array}{l}\text { Fluence } \\
\text { (spectrum) }\end{array}$ & (zero) \\
\hline
\end{tabular}

\section{OT HER FUEL SAMPLE INFORMATION}

Fueled section in each pin was a stack of pellets, with an unenriched pellet at each end. In the central pin, a $10 \%$-enriched pellet was located next to the unenriched pellet. Total of $203 \mathrm{~g}$ of enriched fuel in the test $\sim \sim 29$ $\mathrm{g} / \mathrm{pin})$. Pins were wrapped with $1.6 \mathrm{~mm}$ diameter wire. Central pin received $9 \%$ more power than each edge pin. There was $186 \mathrm{~g}$ of sodium in the test . Ratio of sodium/fueled pin cross-sectional areas $=0.97$

\begin{tabular}{|c|c|}
\hline \multicolumn{2}{|c|}{ TEST CONDITIONS, MEASUREMENTS, \& ANALYSES } \\
\hline $\begin{array}{l}\text { Coolant } \\
\text { environment }\end{array}$ & $\begin{array}{l}\text { Stagnant sodium at } 500-510^{\circ} \mathrm{C} \text { before the power } \\
\text { burst. }\end{array}$ \\
\hline $\begin{array}{l}\text { Transient } \\
\text { conditions }\end{array}$ & $\begin{array}{l}\text { Power burst with } 175 \mathrm{~ms} \text { full-width-at-half- } \\
\text { maximum, generating } 2.15 \mathrm{~kJ} / \mathrm{g} \text {.in the fuel. }\end{array}$ \\
\hline $\begin{array}{l}\text { Fuel sample } \\
\text { configuration } \\
\text { in the test }\end{array}$ & Bunde of 7 wire-wrapped fuel pins. \\
\hline $\begin{array}{l}\text { Test vehicle \& } \\
\text { experiment } \\
\text { system }\end{array}$ & $\begin{array}{l}\text { Piston autoclave vehicle (much different from the } \\
\text { vehicle used in tests } M-2 \text { through } M-6 \text { ) with pin } \\
\text { bundle submerged in sodium inside a Zircaloy } \\
\text { sleeve. }\end{array}$ \\
\hline $\begin{array}{l}\text { Measurements } \\
\text { /diagnostics } \\
\text { during test }\end{array}$ & $\begin{array}{l}\text { Temperatures were measured by four tungsten- } \\
\text { rhenium TCs. (The TCs had time constant = } 15 \\
\text { ms.) Pressure transducer measured autoclave } \\
\text { pressure. Upward motion of a } 260 \mathrm{~g} \text { piston } \\
\text { located immediately above the fuel-pin bundle } \\
\text { was measured by a linear motion transducer. } \\
\text { The TREAT Fast Neutron Hodoscope monitored } \\
\text { fuel motion. }\end{array}$ \\
\hline $\begin{array}{l}\text { Posttest } \\
\text { coolant flow } \\
\text { condition }\end{array}$ & (Not relevant.) \\
\hline $\begin{array}{l}\text { Posttest } \\
\text { condition of } \\
\text { test sample(s) }\end{array}$ & Highly disrupted. \\
\hline $\begin{array}{l}\text { Posttest } \\
\text { measurements } \\
\text { \& information }\end{array}$ & $\begin{array}{l}\text { Neutron radiography of the fuel sample after } \\
\text { each transient. }\end{array}$ \\
\hline $\begin{array}{l}\text { Posttest } \\
\text { analyses }\end{array}$ & Thermal-mechanical and heat transfer analyses. \\
\hline
\end{tabular}

\section{KEY SUMMARY INFORMATION ABOUT THE TEST}

Test S-7, together with test S-8, investigated fuel-coolant interaction effects with FFTF-pin geometry and sodium preheated to $500{ }^{\circ} \mathrm{C}$ (compared to $\sim 200{ }^{\circ} \mathrm{C}$ in the previous S-series tests. Test S-7 was run with a fuel fission energy generation similar to that in test S-3. In $\mathrm{S}-7$, the initial sodium temperature was selected so that sodium boiling would occur before cladding failure. The cladding failed at 1.51 $\mathrm{kJ} / \mathrm{g}$ fuel energy. The largest pressure pulse reached $2.9 \mathrm{MPa}$. The mass-mean fuel debris diameter was about $0.3 \mathrm{~mm}$, similar to that in test S-6. Based on the average fuel energy at initial cladding failure, the calculated energy conversion ratio was $~ 0.0006 \%$. 


\section{Summary of TREAT Test S-8}

Performed 1 October 1971

Transient No. 1403

\section{PRE-TEST SAMPLE CHARACTERIZATION}

\begin{tabular}{|l|l|}
\hline \multicolumn{2}{|c|}{ FUEL } \\
\hline Form & He-bonded $\mathrm{UO}_{2}$ \\
\hline Composition & $\begin{array}{l}\mathrm{UO}_{2}-\text { uranium enrichment } 13 \% \text { in } \\
\text { central pin, 10\% in edge pins }\end{array}$ \\
\hline Geometry & Solid pellet \\
\hline Fissile height & $\sim 13 \mathrm{~cm}$ \\
\hline $\begin{array}{l}\text { Smear } \\
\text { density }\end{array}$ & $85 \%$ of theoretical \\
\hline
\end{tabular}

\begin{tabular}{|l|l|}
\hline \multicolumn{2}{|c|}{ CLADDING } \\
\hline Material & Type 316 stainless steel \\
\hline OD & $5.84 \mathrm{~mm}$ \\
\hline Thickness & $0.38 \mathrm{~mm}$ \\
\hline
\end{tabular}

\begin{tabular}{|l|l|}
\hline \multicolumn{2}{|l|}{ PRE-IRRADIATION CHARACTERISTICS } \\
\hline BU (peak axial) & Zero \\
\hline Peak linear power & (not applicable) \\
\hline $\begin{array}{l}\text { Peak internal clad } \\
\text { temperature }\end{array}$ & (not applicable) \\
\hline $\begin{array}{l}\text { Fluence } \\
\text { (spectrum) }\end{array}$ & (zero) \\
\hline
\end{tabular}

\section{OTHER FUEL SAMPLE INFORMATION}

Fueled section in each pin was a stack of pellets, with an unenriched pellet at each end. In the central pin, a $10 \%$-enriched pellet was located next to the unenriched pellet. Total of $203 \mathrm{~g}$ of fuel in the test. Pins were wrapped with $1.6 \mathrm{~mm}$ diameter wire. Central pin received $9 \%$ more power than each edge pin. There was $138 \mathrm{~g}$ of sodium in the test. Ratio of sodium/fueled pin cross-sectional areas $=1.02$

\begin{tabular}{|c|c|}
\hline \multicolumn{2}{|c|}{ TEST CONDITIONS, MEASUREMENTS, \& ANALYSES } \\
\hline $\begin{array}{l}\text { Coolant } \\
\text { environment }\end{array}$ & $\begin{array}{l}\text { Stagnant sodium at } 500-510^{\circ} \mathrm{C} \text { before the power } \\
\text { burst. }\end{array}$ \\
\hline $\begin{array}{l}\text { Transient } \\
\text { conditions }\end{array}$ & $\begin{array}{l}\text { Power burst with } 140 \mathrm{~ms} \text { full-width-at-half- } \\
\text { maximum, generating } 3.28 \mathrm{~kJ} / \mathrm{g} \text {.in the fuel. }\end{array}$ \\
\hline $\begin{array}{l}\text { Fuel sample } \\
\text { configuration } \\
\text { in the test }\end{array}$ & Bundle of 7 wire-wrapped fuel pins. \\
\hline $\begin{array}{l}\text { Test vehicle \& } \\
\text { experiment } \\
\text { system }\end{array}$ & $\begin{array}{l}\text { Piston autoclave vehicle (much different from the } \\
\text { vehicle used in tests M-2 through M-6) with pin } \\
\text { bunde submerged in sodium inside a Zircal oy } \\
\text { sleeve. }\end{array}$ \\
\hline $\begin{array}{l}\text { Measurements } \\
\text { /diagnostics } \\
\text { during test }\end{array}$ & $\begin{array}{l}\text { Temperatures were measured by four tungsten- } \\
\text { rhenium TCs. (The TCs had time constant = } 15 \\
\text { ms.) Pressure transducer measured autoclave } \\
\text { pressure. Upward motion of a } 260 \mathrm{~g} \text { piston } \\
\text { located immediately above the fuel-pin bunde } \\
\text { was measured by a linear motion transducer. } \\
\text { The TREAT Fast Neutron Hodoscope monitored } \\
\text { fuel motion. }\end{array}$ \\
\hline $\begin{array}{l}\text { Posttest } \\
\text { coolant flow } \\
\text { condition }\end{array}$ & (Not relevant.) \\
\hline $\begin{array}{l}\text { Posttest } \\
\text { condition of } \\
\text { test sample(s) }\end{array}$ & $\begin{array}{l}\text { Highly disrupted, with evidence of high mobility } \\
\text { of molten steel-UO } \mathrm{O}_{2} \text { debris having penetrated } \\
\text { into small spaces. }\end{array}$ \\
\hline $\begin{array}{l}\text { Posttest } \\
\text { measurements } \\
\text { \& information }\end{array}$ & $\begin{array}{l}\text { Neutron radiography of the fuel sample after } \\
\text { each transient. }\end{array}$ \\
\hline $\begin{array}{l}\text { Posttest } \\
\text { analyses }\end{array}$ & Thermal-mechanical and heat transfer analyses. \\
\hline
\end{tabular}

\section{KEY SUMMARY INFORMATION ABOUT THE TEST}

Test S-8, together with test S-7, investigated fuel-coolant interaction effects with FFTF-pin geometry and sodium preheated to $\sim 500{ }^{\circ} \mathrm{C}$ (compared to $\sim 200^{\circ} \mathrm{C}$ in the previous S-series tests.) Test S-8 was run with a fuel fission energy generation similar to that in test S-4. In Test S-8, the initial sodium temperature was selected so that sodium boiling would occur before cladding fail ure. The cladding failed at $1.47 \mathrm{~kJ} / \mathrm{g}$ fuel energy. The largest pressure pulse reached $5.1 \mathrm{MPa}$. Based on the average fuel energy at initial cladding failure, the calculated energy conversion ratio was $0.0006 \%$. The mass-mean fuel debris diameter was about $0.9 \mathrm{~mm}$. 


\section{Summary of TREAT Test S-11

\author{
Performed 31 October 1972
}

Transient No. 1469

\section{PRE-TEST SAMPLE CHARACTERIZATION}

\begin{tabular}{|l|l|}
\hline \multicolumn{2}{|c|}{ FUEL } \\
\hline Form & He-bonded $\mathrm{UO}_{2}$ \\
\hline Composition & $\mathrm{UO}_{2}-13 \%$ enriched U \\
\hline Geometry & Solid pellet (4.93 mm dia.) \\
\hline Fissile height & $15.2 \mathrm{~cm}$ \\
\hline $\begin{array}{l}\text { Smear } \\
\text { density }\end{array}$ & $\mathrm{g} / \mathrm{cm}^{3}$ (92\% of theoretical) \\
\hline
\end{tabular}

\begin{tabular}{|l|l|}
\hline \multicolumn{2}{|c|}{ CLADDING } \\
\hline Material & Type 316 stainless steel, 20\% CW \\
\hline OD & $5.84 \mathrm{~mm}$ \\
\hline Thickness & $0.381 \mathrm{~mm}$ \\
\hline
\end{tabular}

\begin{tabular}{|l|l|}
\hline \multicolumn{2}{|l|}{ PRE-IRRADIATION CHARACTERISTICS } \\
\hline BU (peak axial) & Zero \\
\hline Peak linear power & (not applicable) \\
\hline $\begin{array}{l}\text { Peak internal clad } \\
\text { temperature }\end{array}$ & (not applicable) \\
\hline $\begin{array}{l}\text { Fluence } \\
\text { (spectrum) }\end{array}$ & (zero) \\
\hline
\end{tabular}

\section{OTHER FUEL SAMPLE INFORMATION}

Fueled section in each pin was a stack of pellets, with an unenriched pellet at each end. Fuel mass $=28.4 \mathrm{~g}$. Top and bottom enriched pellets received $14 \%$ more energy than the other enriched pellets. There was $150 \mathrm{~g}$ of sodium in the test.

\begin{tabular}{|c|c|}
\hline \multicolumn{2}{|c|}{ TEST CONDITIONS, MEASUREMENTS, \& ANALYSES } \\
\hline $\begin{array}{l}\text { Coolant } \\
\text { environment }\end{array}$ & $\begin{array}{l}\text { Stagnant sodium at } 140^{\circ} \mathrm{C} \text { and atmospheric } \\
\text { pressure before the power burst. }\end{array}$ \\
\hline $\begin{array}{l}\text { Transient } \\
\text { conditions }\end{array}$ & $\begin{array}{l}\text { Power burst with } 23 \text { ms initial period and } 104 \mathrm{~ms} \\
\text { full-width-at-half-maximum, generating } 7.1 \mathrm{~kJ} / \mathrm{g} \\
\text { average in the test fuel. }\end{array}$ \\
\hline $\begin{array}{l}\text { Fuel sample } \\
\text { configuration } \\
\text { in the test }\end{array}$ & Single fuel pin \\
\hline $\begin{array}{l}\text { Test vehicle \& } \\
\text { experiment } \\
\text { system }\end{array}$ & $\begin{array}{l}\text { Piston autoclave. Fuel pin surrounded by } 2.5 \text { - } \\
\text { mm-thick sodium annulus bounded by a large } \\
\text { molybdenum heat sink. }\end{array}$ \\
\hline $\begin{array}{l}\text { Measurements } \\
\text { /diagnostics } \\
\text { during test }\end{array}$ & $\begin{array}{l}\text { Temperatures were measured by four tungsten- } \\
\text { rhenium TCs. A pressure transducer measured } \\
\text { the autoclave pressure. Upward motion of a } 260 \\
\text { g piston located immediately above the fuel-pin } \\
\text { bundle was measured by a linear motion } \\
\text { transducer. The TREAT Fast Neutron } \\
\text { Hodoscope monitored fuel motion. }\end{array}$ \\
\hline $\begin{array}{l}\text { Posttest } \\
\text { coolant flow } \\
\text { condition }\end{array}$ & (Not relevant.) \\
\hline $\begin{array}{l}\text { Posttest } \\
\text { condition of } \\
\text { test sample(s) }\end{array}$ & Highly disrupted. \\
\hline $\begin{array}{l}\text { Posttest } \\
\text { measurements } \\
\text { \& information }\end{array}$ & $\begin{array}{l}\text { Neutron radiography of the fuel sample after } \\
\text { each transient. }\end{array}$ \\
\hline $\begin{array}{l}\text { Posttest } \\
\text { analyses }\end{array}$ & Thermal-mechanical and heat transfer analyses. \\
\hline
\end{tabular}

\section{KEY SUMMARY INFORMATION ABOUT THE TEST}

Test S-11 was performed to investigate fuel-coolant interactions under extreme conditions of fuel-vapor-driven fragmentation and mixing of molten fuel and sodium. The heat sink and low initial temperature were used to match prompt-burst conditions of fuel, cladding, and codant temperature at the time of cladding breach thus compensating for the power rise rate being much slower than for a prompt burst. The cladding failed when the fuel energy reached $2.22 \mathrm{~kJ} / \mathrm{g}$, about $15.6 \mathrm{~ms}$ before peak power, generating a $\sim 5 \mathrm{~ms}$ wide pressure pulse reaching $15 \mathrm{MPa}$ followed (about $50 \mathrm{~ms}$ after peak power) by a broad pressure pulse of about $130 \mathrm{~ms}$ full width at half maximum with peak value about $13.6 \mathrm{MPa}$. The $\mathrm{UO}_{2}$ vapor pressure at cladding fail ure was estimated to be roughly $6-12 \mathrm{MPa}$. The estimated energy conversion ratio was $0.1 \%$. Analysis suggested that the posttest events observed were due primarily to the action of fuel vapor pressure, and that there was little or no sodium vaporization (presumably because fuel vapor prevented fuel-sodium contact). The observed pressure events indicated that fuel-vapor-driven fuel fragmentation and mixing with sodium (as a possible mechanism for generating an energetic fuel-codant interaction) did not occur. 


\title{
Summary of TREAT Test S-12
}

\author{
Performed 2 November 1972
}

Transient No. 1470

\section{PRE-TEST SAMPLE CHARACTERIZATION}

\begin{tabular}{|l|l|}
\hline \multicolumn{2}{|c|}{ FUEL } \\
\hline Form & He-bonded $\mathrm{UO}_{2}$ \\
\hline Composition & $\mathrm{UO}_{2}-13 \%$ enriched U \\
\hline Geometry & Solid pellet (4.93 mm dia.) \\
\hline Fissile height & $15.2 \mathrm{~cm}$ \\
\hline $\begin{array}{l}\text { Smear } \\
\text { density }\end{array}$ & $\mathrm{g} / \mathrm{cm}^{3}$ (92\% of theoretical) \\
\hline
\end{tabular}

\begin{tabular}{|l|l|}
\hline \multicolumn{2}{|c|}{ CLADDING } \\
\hline Material & Type 316 stainless steel, 20\% CW \\
\hline OD & $6.35 \mathrm{~mm}$ \\
\hline Thickness & $0.635 \mathrm{~mm}$ \\
\hline
\end{tabular}

\begin{tabular}{|l|l|}
\hline \multicolumn{2}{|l|}{ PRE-IRRADIATION CHARACTERISTICS } \\
\hline BU (peak axial) & Zero \\
\hline Peak linear power & (not applicable) \\
\hline $\begin{array}{l}\text { Peak internal clad } \\
\text { temperature }\end{array}$ & (not applicable) \\
\hline $\begin{array}{l}\text { Fluence } \\
\text { (spectrum) }\end{array}$ & (zero) \\
\hline
\end{tabular}

\section{OTHER FUEL SAMPLE INFORMATION}

Fueled section in each pin was a stack of pellets, with an unenriched pellet at each end. Fuel mass $=28.2 \mathrm{~g}$. Top and bottom enriched pellets received $14 \%$ more energy than the other enriched pellets. There was $150 \mathrm{~g}$ of sodium in the test.

\begin{tabular}{|c|c|}
\hline \multicolumn{2}{|c|}{ TEST CONDITIONS, MEASUREMENTS, \& ANALYSES } \\
\hline $\begin{array}{l}\text { Coolant } \\
\text { environment }\end{array}$ & $\begin{array}{l}\text { Stagnant sodium at } 140^{\circ} \mathrm{C} \text { and atmospheric } \\
\text { pressure before the power burst. }\end{array}$ \\
\hline $\begin{array}{l}\text { Transient } \\
\text { conditions }\end{array}$ & $\begin{array}{l}\text { Power burst with } 23 \text { ms initial period and } 104 \mathrm{~ms} \\
\text { full-width-at-half-maximum, generating } 7.1 \mathrm{~kJ} / \mathrm{g} \\
\text { average in the test fuel. }\end{array}$ \\
\hline $\begin{array}{l}\text { Fuel sample } \\
\text { configuration } \\
\text { in the test }\end{array}$ & Single fuel pin \\
\hline $\begin{array}{l}\text { Test vehicle \& } \\
\text { experiment } \\
\text { system }\end{array}$ & $\begin{array}{l}\text { Piston autoclave. Fuel pin surrounded by a } 2.4- \\
\text { mm-thick sodium annulus bounded by a large } \\
\text { molybdenum heat sink. }\end{array}$ \\
\hline $\begin{array}{l}\text { Measurements } \\
\text { /diagnostics } \\
\text { during test }\end{array}$ & $\begin{array}{l}\text { Temperatures were measured by four tungsten- } \\
\text { rhenium TCs. A pressure transducer measured } \\
\text { the autoclave pressure. Upward motion of a } 260 \\
\text { g piston located immediately above the fuel-pin } \\
\text { bundle was measured by a linear motion } \\
\text { transducer. The TREAT Fast Neutron } \\
\text { Hodoscope monitored fuel motion. }\end{array}$ \\
\hline $\begin{array}{l}\text { Posttest } \\
\text { coolant flow } \\
\text { condition }\end{array}$ & (Not relevant.) \\
\hline $\begin{array}{l}\text { Posttest } \\
\text { condition of } \\
\text { test sample(s) }\end{array}$ & Highly disrupted. \\
\hline $\begin{array}{l}\text { Posttest } \\
\text { measurements } \\
\text { \& information }\end{array}$ & $\begin{array}{l}\text { Neutron radiography of the fuel sample after } \\
\text { each transient. }\end{array}$ \\
\hline $\begin{array}{l}\text { Posttest } \\
\text { analyses }\end{array}$ & Thermal-mechanical and heat transfer analyses. \\
\hline
\end{tabular}

\section{KEY SUMMARY INFORMATION ABOUT THE TEST}

Test S-12 was performed to investigate fuel-coolant interactions under extreme conditions of fuel-vapor-driven fragmentation and mixing of molten fuel and sodium. The heat sink and low initial temperature were used to match prompt-burst conditions of fuel, cladding, and codant temperature at the time of cladding breach, thus compensating for the power rise rate being much slower than for a prompt burst. The cladding failed when the fuel energy reached $2.26 \mathrm{~kJ} / \mathrm{g}$, about $14.6 \mathrm{~ms}$ before peak power, indicated by a rapid temperature rise of the sodium and rapid acceleration of the piston, but without a significant pressure rise. The piston movement indicated that the impulse imparted to it was about half as strong as in test S-11. A pressure pulse peaking at $\sim 7 \mathrm{MPa}$ occurred at about peak reactor power. This was followed about 0.36 s later by a set of three 1 -ms-wide pulses, the largest reaching $\sim 20 \mathrm{MPa}$. (similar to the delayed pressure event in test S-11). The $\mathrm{UO}_{2}$ vapor pressure at cladding failure was estimated to be roughly $7-14 \mathrm{MPa}$. The estimated energy conversion ratio was $0.1 \%$. The observed pressure events indicated that fuel-vapor-driven fuel fragmentation and mixing with sodium (as a possible mechanism for generating an energetic fuel-coolant interaction) did not occur 


\section{Appendix S: One-page Summaries of RX-series Tests}

One-page summaries of each of the RX-series tests are included here. These summaries can also be found in TREXR [6]. 


\section{Summary of TREAT Test RX1 \\ Performed 17 December 1982}

Transient No. 2410

\section{PRE-TEST SAMPLE CHARACTERIZATION}

\begin{tabular}{|l|l|}
\hline \multicolumn{2}{|c|}{ FUEL } \\
\hline Form & Hot-pressed urania/sted \\
\hline Composition & $\begin{array}{l}87.6 \mathrm{wt} \% \mathrm{UO}_{2} / 12.4 \mathrm{wt} \% \mathrm{SS} 316, \\
93 \% \text { or } 50 \% \text { enriched U }\end{array}$ \\
\hline Geometry & $\begin{array}{l}\text { Stack of ten 2.33-cm diameter, 1.27- } \\
\text { cm high cylindrical slugs }\end{array}$ \\
\hline Fissile height & $12.7 \mathrm{~cm}$ \\
\hline $\begin{array}{l}\text { Smear } \\
\text { density }\end{array}$ & $\begin{array}{l}\text { Smear density is not applicable. } \\
\text { The slug density was } 9.68 \mathrm{~g} / \mathrm{cm}^{3}\end{array}$ \\
\hline
\end{tabular}

\begin{tabular}{|l|l|}
\hline \multicolumn{2}{|c|}{ CLADDING } \\
\hline Material & No cladding \\
\hline OD & (not applicable) \\
\hline Thickness & (not applicable) \\
\hline
\end{tabular}

PRE-IRRADIATION CHARACTERISTICS

\begin{tabular}{|l|l|}
\hline BU (peak axial) & None \\
\hline Peak linear power & (not applicable) \\
\hline $\begin{array}{l}\text { Peak internal clad } \\
\text { temperature }\end{array}$ & (not applicable) \\
\hline $\begin{array}{l}\text { Fluence } \\
\text { (spectrum) }\end{array}$ & (not applicable) \\
\hline
\end{tabular}

\section{OTHER FUEL SAMPLE INFORMATION}

The top and bottom fuel slugs had $50 \%$ enriched $U$.

The middle eight slugs had $93 \%$ enriched $U$.

Vertical off-center holes in the slugs ( 4 in the top three

slugs, 3 in the fourth slug, 2 in the fifth slug, and 1 in the sixth and seventh slugs, each accommodated one thermocouple. A hole down the center of the top seven slugs accommodated a boron nitride $(\mathrm{BN})$ sheath for a tungsten ultrasonic thermometer probe.

The total $\mathrm{UO}_{2}$ fuel mass was $460 \mathrm{~g}$

\begin{tabular}{|c|c|}
\hline \multicolumn{2}{|c|}{ TEST CONDITIONS, MEASUREMENTS, \& ANALYSES } \\
\hline $\begin{array}{l}\text { Coolant } \\
\text { environment }\end{array}$ & No codant was present. \\
\hline $\begin{array}{l}\text { Transient } \\
\text { conditions }\end{array}$ & $\begin{array}{l}\text { TREAT power rose from } 0 \text { to } 12.8 \mathrm{MW}(\sim 19 \mathrm{~W} / \mathrm{g} \\
\text { UO in the test fuel) in } 4.0 \mathrm{~s} \text {, then was adjusted } \\
\text { to maintain that same power density in the test } \\
\text { fuel during the next } 156 \mathrm{~s} \text {, then scram. }\end{array}$ \\
\hline $\begin{array}{l}\text { Fuel sample } \\
\text { configuration } \\
\text { in the test }\end{array}$ & $\begin{array}{l}\text { Cylindrical stack of fuel slugs } 2.33 \mathrm{~cm} \text { diameter, } \\
12.7 \mathrm{~cm} \text { high. }\end{array}$ \\
\hline $\begin{array}{l}\text { Test vehicle \& } \\
\text { experiment } \\
\text { system }\end{array}$ & $\begin{array}{l}\text { The urania/steel fuel stack was supported on a } \\
\text { depleted urania base below which was a BN } \\
\text { pedestal. The fuel stack was radially surrounded } \\
\text { by a } 9 \% \text { enriched UO } \mathrm{O}_{2} \text { cylinder (nuclear wall, } \\
24.6 \mathrm{~mm} \text { inner diameter, } 4.8 \mathrm{~mm} \text { thick) } \\
\text { extending from the bottom of the fuel stack to } \\
30.5 \mathrm{~cm} \text { above the top of the stack. The nuclear } \\
\text { wall was radially surrounded by heat shields of } \\
\text { tungsten, molybdenum, graphite, and stainless } \\
\text { steel. A void volume of } ~ 5 \text { liters was present } \\
\text { above the fuel. Outside the primary containment } \\
\text { was a dysprosium neutron filter. The test vehicle } \\
\text { was positioned so that the top of the fuel was at } \\
\text { the axial midheight of the TREAT core. }\end{array}$ \\
\hline $\begin{array}{l}\text { Measurements } \\
\text { /diagnostics } \\
\text { during test }\end{array}$ & $\begin{array}{l}\text { Temperatures within and above the fuel (by } \\
\text { various types of thermocouples including a BN- } \\
\text { sheathed ultrasonic thermocouple), system } \\
\text { pressure, fuel motion (hodoscope) }\end{array}$ \\
\hline $\begin{array}{l}\text { Posttest } \\
\text { coolant flow } \\
\text { condition }\end{array}$ & (not applicable) \\
\hline $\begin{array}{l}\text { Posttest } \\
\text { condition of } \\
\text { test sample(s) }\end{array}$ & Melted and axially dispersed \\
\hline $\begin{array}{l}\text { Posttest } \\
\text { measurements } \\
\text { \& information }\end{array}$ & $\begin{array}{l}\text { (apparently no additional posttest examination } \\
\text { was performed) }\end{array}$ \\
\hline $\begin{array}{l}\text { Posttest } \\
\text { analyses }\end{array}$ & $\begin{array}{l}\text { Thermal, thermal-hydraulic (particularly } \\
\text { regarding heating of the BN above and below } \\
\text { the pool) }\end{array}$ \\
\hline
\end{tabular}

\section{KEY SUMMARY INFORMATION ABOUT THE TEST}

Test RX1 investigated the behavior of a molten mixture of $\mathrm{UO}_{2}$ and steel subjected to fission heating in a simulation of the decay-heating stage of the "transition phase" of a hypothetical unprotected LMFBR accident. It was a scoping test designed specifically to focus on the nature of a heat-generating "open pod" of molten fuel and boiling steel. The hodoscope indicated fuel motion began at $\sim 119 \mathrm{~s}$ into the test. Steel vaporization had been predicted to begin at about that time, but the BN sheaths used on the ultrasonic thermometer apparenty began decomposing and generating considerable nitrogen gas at that time, also. The fuel initially moved upward $\sim 4 \mathrm{~cm}$, where it temporarily contacted a 0.25 -mm-thick tantal um foil. At $\sim 131 \mathrm{~s}$, the fuel moved $\sim 8.5 \mathrm{~cm}$ higher where it contacted a similar tantal um foil. Simultaneously, at that time, the BN began a vigorous, constant decomposition producing nitrogen gas apparenty over its outer surface area extending upward at least $15 \mathrm{~cm}$ above the original elevation of the top of the fuel. Between $150-155 \mathrm{~s}$, the fuel reached an elevation $\sim 11 \mathrm{~cm}$ above the initial top-of-fuel location, and by $160 \mathrm{~s}$ it had moved upward an additional $\sim 11 \mathrm{~cm}$. How much of the BN decomposed above the pool versus within the pod, and how much effect the generated nitrogen gas may have had on the overall pool behavior, remained unknown. 


\section{Summary of TREAT Test RX2 \\ Performed 27 May 1983 \\ Transient No. 2450}

\section{PRE-TEST SAMPLE CHARACTERIZATION}

\begin{tabular}{|l|l|}
\hline \multicolumn{2}{|c|}{ FUEL } \\
\hline Form & Hot-pressed urania/sted \\
\hline Composition & $\begin{array}{l}87.6 \mathrm{wt} \% \mathrm{UO}_{2} / 12.4 \mathrm{wt} \% \text { SS316 } \\
93 \% \text { or } 50 \% \text { enriched U } \\
\text { Geometry }\end{array}$ \\
$\begin{array}{l}\text { Stack of ten 2.33-cm diameter, 1.27- } \\
\text { cm high cylindrical slugs }\end{array}$ \\
\hline Fissile height & $12.7 \mathrm{~cm}$ \\
\hline $\begin{array}{l}\text { Smear } \\
\text { density }\end{array}$ & $\begin{array}{l}\text { Smear density is not applicable. } \\
\text { The slug density was } 9.68 \mathrm{~g} / \mathrm{cm}^{3}\end{array}$ \\
\hline
\end{tabular}

\begin{tabular}{|l|l|}
\hline \multicolumn{2}{|c|}{ CLADDING } \\
\hline Material & No cladding \\
\hline OD & (not applicable) \\
\hline Thickness & (not applicable) \\
\hline
\end{tabular}

PRE-IRRADIATION CHARACTERISTICS

\begin{tabular}{|l|l|}
\hline BU (peak axial) & None \\
\hline Peak linear power & (not applicable) \\
\hline $\begin{array}{l}\text { Peak internal clad } \\
\text { temperature }\end{array}$ & (not applicable) \\
\hline $\begin{array}{l}\text { Fluence } \\
\text { (spectrum) }\end{array}$ & (not applicable) \\
\hline
\end{tabular}

\section{OTHER FUEL SAMPLE INFORMATION}

The bottom fuel slugs had $50 \%$ enriched $U$.

The top nine slugs had $93 \%$ enriched $U$.

Vertical holes in the top seven slugs accommodated a total of three thermocouples. The total $\mathrm{UO}_{2}$ fuel mass was $\sim 463 \mathrm{~g}$.

\begin{tabular}{|c|c|}
\hline \multicolumn{2}{|c|}{ TEST CONDITIONS, MEASUREMENTS, \& ANALYSES } \\
\hline $\begin{array}{l}\text { Coolant } \\
\text { environment }\end{array}$ & No codant was present. \\
\hline $\begin{array}{l}\text { Transient } \\
\text { conditions }\end{array}$ & $\begin{array}{l}\text { TREAT power rose from } 0 \text { to } 12.8 \mathrm{MW}(\sim 21 \mathrm{~W} / \mathrm{g} \\
\left.\mathrm{UO}_{2} \text { in the test fuel }\right) \text { in } 4.0 \mathrm{~s} \text {, then was adjusted } \\
\text { to maintain that power density in the test fuel } \\
\text { during the next } 154 \mathrm{~s} \text {, then scram. }\end{array}$ \\
\hline $\begin{array}{l}\text { Fuel sample } \\
\text { configuration } \\
\text { in the test }\end{array}$ & $\begin{array}{l}\text { Cylindrical stack of fuel slugs } 2.33 \mathrm{~cm} \text { diameter, } \\
12.7 \mathrm{~cm} \text { high. }\end{array}$ \\
\hline $\begin{array}{l}\text { Test vehicle \& } \\
\text { experiment } \\
\text { system }\end{array}$ & $\begin{array}{l}\text { The urania/steel fuel stack was supported on a } \\
\text { depleted urania base below which was a BN } \\
\text { pedestal. The fuel stack was radially surrounded } \\
\text { by a } 9 \% \text { enriched } \mathrm{UO}_{2} \text { cylinder (nuclear wall, } \\
24.6 \mathrm{~mm} \text { inner diameter, } 4.8 \text { mm thick) } \\
\text { extending from the bottom of the fuel stack to } \\
17.8 \mathrm{~cm} \text { above the top of the stack. The nuclear } \\
\text { wall was radially surrounded by heat shields of } \\
\text { tungsten, molybdenum, graphite, and stainless } \\
\text { steel. A void volume of } ~ 0.5 \text { liter was present } \\
\text { above the fuel. Outside the primary containment } \\
\text { was a dysprosium neutron filter. The test vehicle } \\
\text { was positioned so that the top of the fuel was at } \\
\text { the axial midheight of the TREAT core. }\end{array}$ \\
\hline $\begin{array}{l}\text { Measurements } \\
\text { /diagnostics } \\
\text { during test }\end{array}$ & $\begin{array}{l}\text { Temperatures at various locations in and above } \\
\text { the fuel, system pressure, fuel motion } \\
\text { (hodoscope) [No BN-sheathed ultrasonic } \\
\text { thermometer was present.] }\end{array}$ \\
\hline $\begin{array}{l}\text { Posttest } \\
\text { coolant flow } \\
\text { condition }\end{array}$ & (not applicable) \\
\hline $\begin{array}{l}\text { Posttest } \\
\text { condition of } \\
\text { test sample(s) }\end{array}$ & Melted; radially and axially dispersed \\
\hline $\begin{array}{l}\text { Posttest } \\
\text { measurements } \\
\text { \& information }\end{array}$ & $\begin{array}{l}\text { Neutron radiography (apparently no additional } \\
\text { posttest examination was performed) }\end{array}$ \\
\hline $\begin{array}{l}\text { Posttest } \\
\text { analyses }\end{array}$ & Thermal, thermal-hydraulic \\
\hline
\end{tabular}

\section{KEY SUMMARY INFORMATION ABOUT THE TEST}

Test RX2 was designed to examine the behavior of the "debris pool" of molten urania fuel and boiling steel in a confined volume having void volume about ten times the pod volume. The hodoscope indicated gradual upward movement of the top of the fuel by $1.6 \mathrm{~cm}$ during the first $90 \mathrm{~s}$ of the test. The largest mass of fuel movement occurred between 109 and $120 \mathrm{~s}$ into the test, with the upper quarter of the fuel column relocating radially outward and downward. (This movement was confirmed by posttest radiography as having been molten fuel and steel breaching the surrounding nuclear heated wall and lodging in the annulus between the heated wall and the tungsten heat shield.) During the next $50 \mathrm{~s}$, the hodoscope data indicated that fuel continued to redistribute to the lower annular region between the nuclear-heated wall and the tungsten heat shield, and a small amount of fuel accumulated about $12 \mathrm{~cm}$ above the top of the original fuel column. 


\section{Appendix T: One-page Summaries of J-series Tests}

One-page summaries of each of the J-series tests are included here. These summaries can also be found in TREXR [6]. 


\title{
Summary of TREAT Test J1
}

\author{
Performed 21 September 1979
}

Transient No. 2159

\section{PRE-TEST SAMPLE CHARACTERIZATION}

\begin{tabular}{|l|l|}
\hline \multicolumn{2}{|c|}{ FUEL } \\
\hline Form & MOX fuel helium-bonded to cladding \\
\hline Composition & $\begin{array}{l}75 \% \mathrm{UO}_{2}-25 \% \mathrm{PuO}_{2} \\
79 \text { at } \% \text { enriched } \mathrm{U} ; \mathrm{O} / \mathrm{M}=1.96\end{array}$ \\
\hline Geometry & Dished pellets in stack \\
\hline Fissile height & $34.3 \mathrm{~cm}$ \\
\hline $\begin{array}{l}\text { Smear } \\
\text { density }\end{array}$ & $9.34 \mathrm{~g} / \mathrm{cm}^{3}$ \\
\hline
\end{tabular}

\begin{tabular}{|l|l|}
\hline \multicolumn{2}{|c|}{ CLADDING } \\
\hline Material & 316 stainless steel, 20\% cold-worked \\
\hline OD & $5.84 \mathrm{~mm}$ \\
\hline Thickness & $0.38 \mathrm{~mm}$ \\
\hline
\end{tabular}

\begin{tabular}{|l|l|}
\hline \multicolumn{2}{|l|}{ PRE-IRRADIATION CHARACTERISTICS } \\
\hline BU (peak axial) & $\sim 7$ at\% \\
\hline Peak linear power & $26 \mathrm{~kW} / \mathrm{m}$ \\
\hline $\begin{array}{l}\text { Peak internal clad } \\
\text { temperature }\end{array}$ & (not available) \\
\hline $\begin{array}{l}\text { Fluence } \\
\text { (spectrum) }\end{array}$ & $\begin{array}{l}\text { Fluence not available. } \\
\text { Fast (EBR-II) spectrum. }\end{array}$ \\
\hline
\end{tabular}

\section{OTHER FUEL SAMPLE INFORMATION}

Fuel mass per pin $=64.3 \mathrm{~g}$. Fuel pins were wire wrapped with 1.1-mm diameter wire. Above the fuel was $12.3 \mathrm{~cm}$ of $\mathrm{UO}_{2}$ insulator, then a $14.5-\mathrm{cm}$-long $4.8-\mathrm{mm}$ diameter Inconel reflector.

\begin{tabular}{|l|l|}
\hline \multicolumn{2}{|c|}{ TEST CONDITIONS, MEASUREMENTS, \& ANALYSES } \\
\hline $\begin{array}{l}\text { Coolant } \\
\text { environment }\end{array}$ & $\begin{array}{l}\text { Flowing sodium set at } 610 \mathrm{~cm} / \mathrm{s} \text { ( } 940 \mathrm{~cm} / \mathrm{s} \text { ) and } \\
475{ }^{\circ} \mathrm{C}\end{array}$ \\
\hline $\begin{array}{l}\text { Transient } \\
\text { conditions } \\
\text { in the edge pins), then linear decrease for } 1.3 \mathrm{~s} \\
\text { to } 169 \mathrm{MW} \text {, then linear increase for } 3.5 \mathrm{~s} \text { to } 255 \\
\text { MW peak power (at } \mathrm{t}=9.12 \mathrm{~s} \text { ), then linear } \\
\text { decrease for } 0.2 \mathrm{~s} \text { to } 30 \mathrm{MW} \text {, then scram } 0.9 \mathrm{~s} \\
\text { later. Ratio of central pin power to edge pin } \\
\text { power was } 81 \% .\end{array}$ \\
\hline $\begin{array}{l}\text { Fuel sample } \\
\text { configuration } \\
\text { in the test }\end{array}$ & Seven-pin bundle of pre-irradiated pins \\
\hline $\begin{array}{l}\text { Test vehicle \& } \\
\text { experiment } \\
\text { system }\end{array}$ & $\begin{array}{l}\text { Mark-IIC sodium loop with neutron filtering and } \\
\text { axial-flux-shaping collars; fluted stainless sted } \\
\text { flowtube surrounded by inert-gas annul us. }\end{array}$ \\
\hline $\begin{array}{l}\text { Measurements } \\
\text { /diagnostics } \\
\text { during test }\end{array}$ & $\begin{array}{l}\text { Temperatures, inlet and outlet flow rates, inlet } \\
\text { and outtet pressures, flowtube strains }\end{array}$ \\
\hline $\begin{array}{l}\text { Posttest } \\
\text { coolant flow } \\
\text { condition }\end{array}$ & $\begin{array}{l}\text { Variable, increasing after scram from a low of } \\
20 \% \text { of the initial flow rate to a final value of } 70 \% \\
\text { of the initial flow rate. }\end{array}$ \\
\hline $\begin{array}{l}\text { Posttest } \\
\text { condition of } \\
\text { test sample(s) }\end{array}$ & Three of the four pins had mildly failed. \\
\hline $\begin{array}{l}\text { Posttest } \\
\text { measurements } \\
\text { \& information }\end{array}$ & $\begin{array}{l}\text { Neutron radiography. macro- and micro- } \\
\text { examinations. }\end{array}$ \\
\hline $\begin{array}{l}\text { Posttest } \\
\text { analyses }\end{array}$ & Thermal-hydraulic; fuel transient behavior \\
\hline
\end{tabular}

KEY SUMMARY INFORMATION ABOUT THE TEST

Test J1 showed that a slow-period LMFBR transient overpower simulation causing cladding failure in an FFTF-like fuel pin could be performed within the limitations of the TREAT reactor. Fuel pin failure occurred at $9.035 \mathrm{~s}$ (252 MW TREAT power), causing the inlet flow rate to suddenly decrease by $25 \%$ (not enough to trigger the programmed TREAT power drop). A second, stronger flow event occurred $40 \mathrm{~ms}$ later, causing (within $50 \mathrm{~ms}$ ) the inlet flow rate to decrease to about $44 \%$ of its original value (triggering the power drop) and the outlet flow rate to triple. During the next $\sim 100 \mathrm{~ms}$, the fuel region began refilling with sodium, and the inlet and outlet flow rates both reached $80-90 \%$ of their initial values. Then the flow rates began dropping to a temporary hold at $\sim 60 \%$ of initial value, then dropping steadily to $\sim 20 \%$ of initial as the reactor was scramming at $t=10.26 \mathrm{~s}$. Beginning at $\mathrm{t}=\sim 15 \mathrm{~s}$, the inlet and outlet flow rates began a steady, linear increase reaching $70-75 \%$ at $t=120 \mathrm{~s}$. (The cause of this late flow behavior was apparenty never determined.) Posttest examination showed that the fuel columns of some fuel pins had elongated, from 0.2 to $3.2 \mathrm{~cm}$ in the edge pins (higher values corresponding to higher relative pin power during the test) and $0.2 \mathrm{~cm}$ in the center pin. The largest expansions almost fully compressed the plenum spring. Up to about $30 \%$ areal fuel melting occurred in the pins, and central voids had formed in all of the edge pins. Major failures occurred in three pins (two of the hotter edge pins and one of the cooler edge pins); these were the only pins to release fuel. The breaches were all at the top of the fuel column and extended up along the insulator pellet stacks. The breaches were up to $\sim 3 \mathrm{~cm}$ long and involved up to half the circumference of the cladding. Practically all of the fuel that had melted in the failed pins was released from those pins. Much of the released fuel (estimated to be $53 \mathrm{~g}$ total) stayed near the failure sites. No significant blockage to coolant flow was evident in the region occupied by the fuel pins. Only a small amount of the fuel in the center pin had melted, and there was a small crack (adjacent to an insulator pellet) in the pin's cladding. The flowtube had remained intact. 


\section{Argonne $\mathbf{A}$}

Nuclear Science and Engineering Division

Argonne National Laboratory

9700 South Cass Avenue, Bldg. 208

Argonne, IL 60439

www.anl.gov

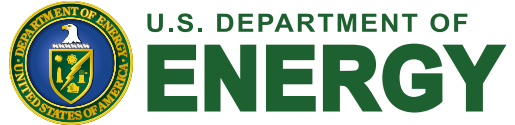

Argonne National Laboratory is a U.S. Department of Energy laboratory managed by UChicago Argonne, LLC 